\author{
BOSTON UNIVERSITY \\ GRADUATE SCHOOL OF ARTS AND SCIENCES
}

Dissertation

\title{
MUON SPIN PRECESSION FREQUENCY EXTRACTION \\ AND DECAY POSITRON TRACK FITTING IN RUN 1 \\ OF THE FERMILAB MUON $g-2$ EXPERIMENT
}

by

\section{NICHOLAS BRENNAN KINNAIRD \\ B.S., University of Texas at Austin, 2013 \\ B.S., University of Texas at Austin, 2013 \\ M.A., Boston University, 2016}

Submitted in partial fulfillment of the

requirements for the degree of

Doctor of Philosophy 
(C) 2020 by NICHOLAS BRENNAN KINNAIRD All rights reserved 


\section{Approved by}

First Reader

B. L. Roberts, PhD

Professor of Physics

Second Reader

R. M. Carey, PhD

Professor of Physics 


\section{Dedication}

I dedicate this thesis to my parents, John, Nett, and Zindy. Thank you for all the support and encouragement along the way. I love you all dearly. 


\section{Acknowledgments}

There are many people I would like to thank in my journey as a graduate student. First and foremost, my advisor Lee Roberts, for whom it has been an absolute pleasure and honor to work for. He gave me a special and unique opportunity to join the BU physics group and E989 collaboration, and I will always cherish it. To my second reader of this dissertation, Rob Carey, who has taught me many things along the way, including introducing me to the track fitting which was my first major project on the experiment. Thanks also to Jim Miller, who has always provided advice and suggestions regarding my work. Thanks to my non $g-2$ committee members Ken Lane and Shyam Erramilli, who taught me particle theory and electromagnetism respectively, without which I would not have understood the experiment I was on. Much thanks to Mirtha Cabello, the BU physics graduate student coordinator, who was always a joy to talk to and who was invaluable when it came to dealing with all of the administrative stuff. Thanks to my BU office buddy and friend, John Quirk, who was always a great source of humor and grounding when things got tough. I would also like to give special thanks to James Mott, who had endless patience and support for me as I grew, both as a graduate student and a person. He was a fantastic advisor to me as I lived in Fermilab these last few years, and being able to turn my chair around and ask for advice saved my graduate career on more than occasion.

I'd like to thank the entire E989 collaboration, without whom none of this work would have been possible. I'd first like to thank the Tracking team, of which I was a part for many years. Thanks to Brendan Casey, James Mott, Mark Lancaster, Will Turner, Saskia Charity, Joe Price, Barry King, Becky Chislett, Tabitha HalewoodLeagas, Talal Albahri, Tammy Walton, and Gleb Lukicov. I'm not sure there's a better group to have been a part of in all of particle physics to be honest. Special thanks

again to Will and Gleb, who were my closest friends while at Fermilab, and were 
always a great source of support and camaraderie. Thanks also to Aaron Fienberg and David Sweigart, who were fantastic peers in the precession frequency analysis, and who I will always strive to emulate in the way I approach my work. Thanks also to Alex Keshavarzi, Sudeshna Ganguly, Anna Driutti, Leah Welty-Rieger, Midhat Farooq, Joe Grange, Jason Hempstead, Rachel Osofsky, James Stapleton, Nam Tran, Yaqian Wang, and many, many more who provided advice, help, and friendship along the way.

I would also like to thank my undergraduate advisor Karol Lang and Marek Proga from the University of Texas at Austin, for whom I really got started in the field of particle physics many years ago.

Lastly, I'd like to thank my family. Thanks to my parents, John, Nett and Zindy, my sister Maris, and my brothers River and Aidan. I have received endless love and support from them over the years, and I am an extraordinarily lucky person to have the family that I do. Finally, to my love, Jenny Mahon, who has made the last year of my graduate school career the best one yet, and with whom I'm excited to share many future adventures with.

My participation in this work was supported in part by the U.S. Department of Science Office of High Energy Physics Award DE-SC0013895. 


\title{
MUON SPIN PRECESSION FREQUENCY EXTRACTION AND DECAY POSITRON TRACK FITTING IN RUN 1 OF THE FERMILAB MUON $g-2$ EXPERIMENT
}

\section{NICHOLAS BRENNAN KINNAIRD}

\author{
Boston University, Graduate School of Arts and Sciences, 2020 \\ Major Professor: B. L. Roberts, Professor of Physics
}

\begin{abstract}
One of the few indications for new physics is the discrepancy between the theoretical and experimental values for the anomalous magnetic moment of the muon. There is a discrepancy of 3 to 4 standard deviations between theory and the last experimental measurement made at Brookhaven National Laboratory in 2001, which measured the muon magnetic anomaly $a_{\mu}$ to 540 parts per billion (ppb). This discrepancy has been consistent for many years with ever improving theoretical calculations. In order to resolve or confirm this discrepancy experiment E989, Muon $g-2$, is underway to measure $a_{\mu}$ to 4 times higher precision at $140 \mathrm{ppb}$. In Run 1 E989 gathered its first production data, consisting of approximately $8 \times 10^{9}$ decay positrons above an energy threshold of $1.7 \mathrm{GeV}$.

This dissertation describes the experimental measurement, the detectors, the precession frequency extraction, and the track fitting of decay positrons in Run 1. The track fitting is done using a $\chi^{2}$ minimization algorithm to fit tracks propagated within a Geant4 reconstruction simulation including error propagation. The precession frequency is extracted using an analysis technique called the Ratio Method. The Ratio


Method takes the ratio of time-shifted decay positron spectra in order to remove the decay exponential along with slowly varying effects in the data. Precession frequency extraction analyses for four near-final Run 1 datasets are presented with full systematic error evaluations. The total Run 1 precession frequency error determined in this analysis is $469.4 \mathrm{ppb}$, where the error is statistics dominated. Combined with the expected error in the magnetic field measurement of $140 \mathrm{ppb}$, the expected final error on $a_{\mu}$ for Run 1 of E989 is $\mathcal{O}(500 \mathrm{ppb})$, comparable to the previous measurement. 


\section{Contents}

1 Introduction 1

1.1 Magnetic moments of particles . . . . . . . . . . . . . . . . . 2

1.2 Standard Model contributions to $a_{\mu} \ldots \ldots \ldots$. . . . . . . . . 3

1.2 .1 QED . . . . . . . . . . . . . . . . . . . 4

1.2 .2 Electroweak . . . . . . . . . . . . . . . . 5

1.2 .3 Hadronic . . . . . . . . . . . . . . . . . 6

1.2 .4 Combined Standard Model value . . . . . . . . . . . . . . . . 9

$1.3 \quad$ Experimental value of $a_{\mu}$ and discrepancy with $a_{\mu}^{\mathrm{SM}} \ldots \ldots . . . . . \quad 10$

1.4 Beyond the Standard Model and the purpose of E989 . . . . . . . . . 11

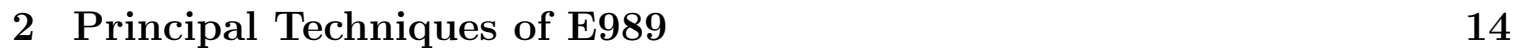

2.1 Measuring $\omega_{a} \ldots \ldots \ldots \ldots$

2.2 Measuring the magnetic field . . . . . . . . . . . . . . . . . . . . 21

2.3 Production of polarized muons . . . . . . . . . . . . . . . . . . 25

2.4 Injection of muons $\ldots \ldots \ldots \ldots$

2.5 Storage of muons . . . . . . . . . . . . . . . . . . . . . . . 31

2.5 .1 Kicker . . . . . . . . . . . . . . . . . . 33

2.5 .2 Electrostatic quadrupoles . . . . . . . . . . . . . 33

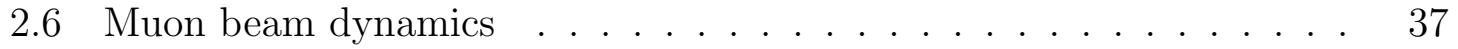

2.6 .1 Coherent betatron oscillation . . . . . . . . . . . . . . 39

2.6 .2 Beam debunching . . . . . . . . . . . . . . . . 41

2.7 Corrections to $\omega_{a} \ldots \ldots \ldots \ldots$. . . . . . . . . . . . . . . . . . . . 42 
2.7 .1 Electric field correction . . . . . . . . . . . . . . . . . . 44

2.7 .2 Pitch correction . . . . . . . . . . . . . . . . . 46

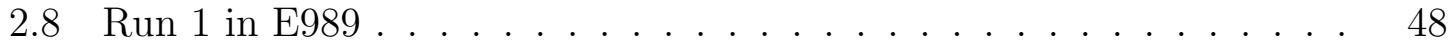

\begin{tabular}{lll}
\hline & Detector Systems & 52
\end{tabular}

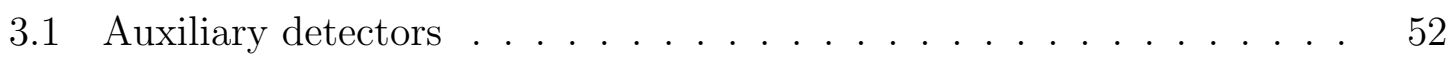

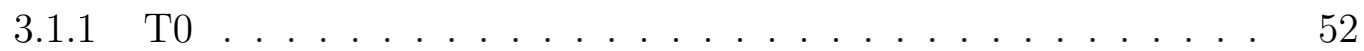

3.1 .2 Inflector Beam Monitoring System … . . . . . . . 53

$3.1 .3 \quad$ Fiber harps $\ldots \ldots \ldots \ldots \ldots \ldots \ldots$

3.2 Calorimeters . . . . . . . . . . . . . . . . . . . . 56

$3.2 .1 \quad$ Requirements and systematic effects $\ldots \ldots \ldots \ldots$

3.2 .2 Hardware . . . . . . . . . . . . . . . . . . 57

$3.2 .3 \quad$ Laser calibration system $\ldots \ldots \ldots \ldots$

3.3 Straw trackers $\ldots \ldots \ldots \ldots \ldots$

3.3 .1 Tracker readout electronics. . . . . . . . . . . . . . . . . 64

\begin{tabular}{|lll}
4 & Track Reconstruction and Analysis & 70
\end{tabular}

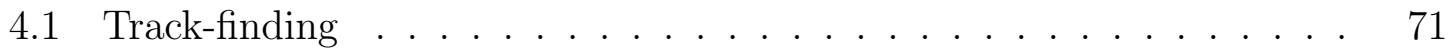

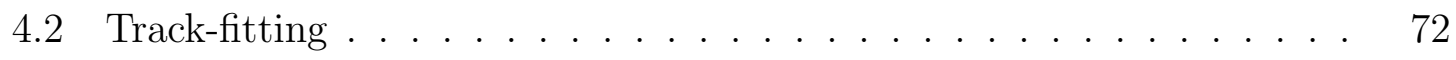

$4.2 .1 \quad$ Error propagation and coordinate systems $\ldots \ldots \ldots$. . . . 73

$4.2 .2 \quad \chi^{2}$ minimization $\ldots \ldots \ldots \ldots \ldots \ldots$

4.2 .3 Fits to idealized tracks in vacuum . . . . . . . . . . . 84

4.2 .4 Material correlations . . . . . . . . . . . . . 85

$4.2 .5 \quad$ Fits to simulated tracks including material effects . . . . . 89

4.2 .6 Left-right ambiguity and fit modes $\ldots \ldots \ldots \ldots$

4.2.7 $\quad$ Fits to tracks from data and comparison with Monte-Carlo . . 98

4.3 Track extrapolation . . . . . . . . . . . . . . . . . . . . . . . . . . . . 101

4.4 Muon beam measurements . . . . . . . . . . . . . . . . . . . . . . . 101 
$\begin{array}{lll}5 & \omega_{a} \text { Measurement } & 109\end{array}$

5.1 Reconstruction of decay positron hits $\ldots \ldots \ldots \ldots$

5.2 Construction of positron hit energy and time spectra $\ldots \ldots \ldots \ldots$

5.2 .1 Pileup subtraction . . . . . . . . . . . . . . . . . . . . . 118

5.2 .2 Ratio Method . . . . . . . . . . . . . . . . . 126

$5.3 \quad$ Fitting the data $\ldots \ldots \ldots \ldots \ldots \ldots \ldots \ldots \ldots$

$5.3 .1 \quad$ CBO terms $\ldots \ldots \ldots \ldots \ldots \ldots \ldots \ldots$

$5.3 .2 \quad \mathrm{vW}$ term $\ldots \ldots \ldots \ldots \ldots \ldots \ldots \ldots$

5.3 .3 Lost muons . . . . . . . . . . . . . . . . . . . . . . . . . 140

5.3 .4 Fit procedures and parameters . . . . . . . . . . . . . . . 147

5.4 Fit results . . . . . . . . . . . . . . . . . . . . . . . . . . . . . . 149

5.4 .1 Individual calorimeter fits . . . . . . . . . . . . . . . 154

5.4.2 Scans over fit start time, fit end time, and energy threshold. . 157

5.4 .3 Fits to bunch number. . . . . . . . . . . . . . . . . 163

5.4 .4 Fits to many random seeds . . . . . . . . . . . . . 165

5.5 Systematic errors $\ldots \ldots \ldots \ldots \ldots \ldots \ldots$

5.5 .1 Pileup systematic errors _. . . . . . . . . . . . . . . 168

5.5 .2 Gain systematic errors $\ldots \ldots \ldots \ldots$

$5.5 .3 \quad$ CBO systematic errors $\ldots \ldots \ldots \ldots \ldots \ldots$

5.5 .4 Lost muon systematic errors . . . . . . . . . . . . . 188

$5.5 .5 \quad$ Ratio construction systematic errors $\ldots \ldots \ldots \ldots$

$5.5 .6 \quad$ Binning systematic errors $\ldots \ldots \ldots \ldots$

$5.5 .7 \quad$ Systematic error from differential decay . . . . . . . . . . . . 198

5.5 .8 Systematic errors in the E-field and pitch corrections . . . . . 199

5.5 .9 Systematic error due to vertical beam motion . . . . . . . . 201

5.5 .10 Systematic error summary . . . . . . . . . . . . . . . 202 
$5.6 \quad$ Final precession frequency results . . . . . . . . . . . . . . . . . 204

$\begin{array}{lll}6 & \text { Conclusion } & 206\end{array}$

\begin{tabular}{|lr}
\hline A Tracking Derivations & 210
\end{tabular}

A.1 Straw measurement angular correction . . . . . . . . . . . . . . . . . 210

A.2 Track fitting correlation matrices . . . . . . . . . . . . . . . 213

\begin{tabular}{|ll}
\hline B Ratio Method Derivation & 214
\end{tabular}

B.1 Ratio form and function . . . . . . . . . . . . . . . . . 214

B.2 Ratio errors . . . . . . . . . . . . . . . . . . . . . . . . 219

\begin{tabular}{ll}
\hline C Pileup Modified Errors & 220
\end{tabular}

C.1 Pileup errors for the ratio function . . . . . . . . . . . . . . . 223

D Fit Result Correlation Matrices $\quad 225$

E Ratio Method - T-Method Randomization Comparison 230

\begin{tabular}{ll}
\hline Bibliography & 235
\end{tabular}

$\begin{array}{ll}\text { Curriculum Vitae } & 245\end{array}$ 


\section{List of Tables}

2.1 Uncertainties in the magnetic field measurement . . . . . . . . . . . . 26

2.2 Muon beam frequencies in the E989 experiment . . . . . . . . . . . . 38

2.3 Run 1 datasets . . . . . . . . . . . . . . . . . . 50

4.1 Run 1 dataset muon beam parameters . . . . . . . . . . . . . 104

$5.1 \quad$ Parameters used in the construction of $\omega_{a}$ time spectra . . . . . . . 115

5.2 Dataset CBO frequency model parameters . . . . . . . . . . . . 136

5.3 Asymmetry values in Run 1 datasets with VW time randomization . 139

5.4 Lost muon cuts . . . . . . . . . . . . . . . . . . . . . . . . . . . . . . 142

5.5 Fit procedure parameters . . . . . . . . . . . . . . . . . . . . . . . . 148

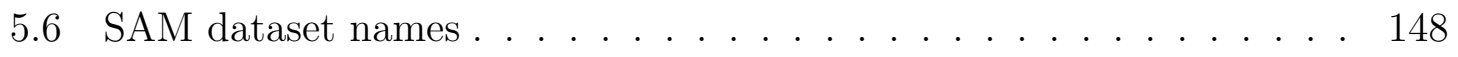

$5.7 \quad$ Fit results for Run 1 precession frequency analysis datasets . . . . . . 151

5.8 Random seed fit results . . . . . . . . . . . . . . . . . . . . . . 165

5.9 Target systematic uncertainties in the precession frequency measurement 167

5.10 Systematic error due to pileup amplitude . . . . . . . . . . . . . . . . 174

5.11 Systematic error due to pileup time-shift . . . . . . . . . . . . . . . 176

5.12 Systematic error due to pileup energy scale . . . . . . . . . . . . . . . 177

5.13 Total pileup-related systematic errors . . . . . . . . . . . . . . . . . 178

5.14 Systematic error due to IFG amplitude . . . . . . . . . . . . . . . . . 181

5.15 Systematic error due to IFG lifetime . . . . . . . . . . . . . . . . . . 182

5.16 Systematic error due to STDP . . . . . . . . . . . . . . . 185

5.17 Total gain-related systematic errors . . . . . . . . . . . . . . 185 
5.18 Changes in $R$ with tracker station 18 CBO frequency model parameters 186

5.19 Systematic error due to CBO envelope . . . . . . . . . . . . . . . . . 188

5.20 Total CBO-related systematic errors . . . . . . . . . . . . . . . . 188

5.21 Effect on fitted $\mathrm{R}$ value due to lost muon cuts in the 9d and Endgame datasets . . . . . . . . . . . . . . . . . . . . 189

5.22 Systematic error due to fixed $\kappa_{\text {loss }}$. . . . . . . . . . . . . . . . . . . 191

5.23 Systematic error due to lost muon phase bias . . . . . . . . . . . . . . 193

5.24 Sensitivities of $R$ to ratio construction parameters . . . . . . . . . . 196

5.25 Sensitivities of $R$ to binning parameters . . . . . . . . . . . . . . . 198

5.26 Electric field corrections estimated by the Cornell fast rotation analysis group . . . . . . . . . . . . . . . . . . . 200

5.27 Systematic errors evaluated in the Run 1 precession frequency datasets 203

5.28 Run 1 precession frequency final results . . . . . . . . . . . . . . . 205

C.1 Number of doublet counts added to pileup histogram from shadow method . . . . . . . . . . . . . . . . 221

E.1 T-R Method randomization comparison . . . . . . . . . . . . . . . . 233 


\section{List of Figures}

$1 \cdot 1 \quad$ QED diagrams contributing to the magnetic moment . . . . . . . . 4

$1 \cdot 2 \quad$ Electroweak diagrams contributing to the magnetic moment . . . . . 5

$1 \cdot 3 \quad$ First order hadronic vacuum polarization diagram contributing to the magnetic moment . . . . . . . . . . . . . . . . . . . . . . . . . . . . . 7

$1 \cdot 4 \quad$ Hadronic light-by-light diagrams contributing to the magnetic moment 9

$1.5 \quad$ Comparison between theoretical and experimental values for $a_{\mu} \ldots$. . 10

$1.6 \quad$ Citations for E821 publications vs year . . . . . . . . . . . . . 13

$2 \cdot 1 \quad$ Diagram for muon decay $\ldots \ldots \ldots \ldots$. . . . . . . . . . . . 17

$2 \cdot 2 \quad$ Muon decay for maximum and minimum energy decay positrons . . . 17

$2 \cdot 3 \quad$ Number distribution and asymmetry for muon decay in the muon rest frame and lab frame . . . . . . . . . . . . . . . . . . . . . . . . . . . 19

$2 \cdot 4 \quad g-2$ time spectrum example . . . . . . . . . . . . . 20

2.5 Magnet cross section . . . . . . . . . . . . . . . . . . . . 22

$2 \cdot 6 \quad$ FID signal $\ldots \ldots \ldots \ldots \ldots$

$2 \cdot 7 \quad$ Azimuthally averaged magnetic field sample . . . . . . . . . . . . . 24

$2 \cdot 8 \quad$ Fermilab accelerator layout for muon delivery to E989 . . . . . . . . . 28

$2.9 \quad$ Fermilab accelerator pulse train . . . . . . . . . . . . . . . . . . 28

$2 \cdot 10$ Muon injection point through inflector . . . . . . . . . . . 30

$2 \cdot 11$ Superconducting inflector magnet . . . . . . . . . . . . . 31

$2 \cdot 12$ The E989 experiment . . . . . . . . . . . . . . . . . . . . . . . . . 32

$2 \cdot 13$ Vacuum chamber map . . . . . . . . . . . . . . . 34 
$2 \cdot 14$ Electrostatic quadrupoles installed in a vacuum chamber . . . . . . . 35

$2 \cdot 15$ Electrostatic quadrupole potentials . . . . . . . . . . . . . 36

$2 \cdot 16$ Tune plane . . . . . . . . . . . . . . . . . . . . . . 39

$2 \cdot 17$ Coherent betatron oscillation . . . . . . . . . . . . . . 41

$2 \cdot 18$ Beam debunching fast rotation . . . . . . . . . . . . 43

$2 \cdot 19$ Pitching beam motion . . . . . . . . . . . . . . . . 47

$2 \cdot 20$ Electrostatic quadrupole high voltage traces . . . . . . . . . . . . 51

3.1 $\quad$ T0 counter and pulses $\ldots \ldots \ldots \ldots$. . . . . . . . . . . . . 53

$3 \cdot 2$ IBMS models . . . . . . . . . . . . . . . . . . . . . . 54

$3 \cdot 3$ IBMS positions $\ldots \ldots \ldots \ldots$. . . . . . . . . . . . . . . . 54

$3.4 \quad$ Fiber harp and measurement . . . . . . . . . . . . . . . 55

3.5 $\quad$ Calorimeters . . . . . . . . . . . . . . . . . . . . . . . . . . . 58

$3.6 \quad$ Straw tracker drift circles . . . . . . . . . . . . . . . . . . . . . . . 62

3.7 Tracker module . . . . . . . . . . . . . . . . . 63

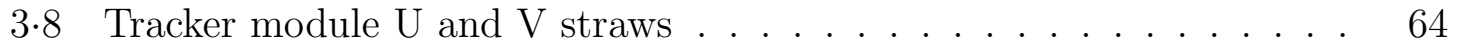

3.9 Model view of tracker station . . . . . . . . . . . . . . . . . 65

$3 \cdot 10$ Muon perspective view of tracker station . . . . . . . . . . 65

$3 \cdot 11$ Tracker ASDQs and associated components . . . . . . . . . . . . . . 67

$3 \cdot 12$ Tracker feedthough board . . . . . . . . . . . . . . . . 68

3.13 Tracker readout boards and housing . . . . . . . . . . . . . . 68

$3 \cdot 14$ Front-end tracker electronics readout chain . . . . . . . . . . . . . . . 69

4.1 Track candidate selection . . . . . . . . . . . . . . . . . . 72

$4 \cdot 2 \quad$ Vertical and radial magnetic fields calculated in Opera2D . . . . . . . 74

$4 \cdot 3 \quad$ Free and surface tracking coordinate systems . . . . . . . . . . . . 76

4.4 Tracker reference frame . . . . . . . . . . . . . . . . . . . . . . 76

4.5 Transport and error matrices for straw tracker planes . . . . . . . . . 79 
$4 \cdot 6 \quad$ Natural tracker measurement system . . . . . . . . . . . . . . . . . . 80

$4 \cdot 7 \quad$ p-value and $\chi^{2}$ distribution for fitted tracks in vacuum . . . . . . 85

4.8 Track parameter truth pulls for fitted tracks in vacuum . . . . . . . . 86

4.9 Material scattering error in tracker . . . . . . . . . . . . . . . . . 87

$4 \cdot 10 \mathrm{p}$-value and $\chi^{2}$ distribution for fitted tracks in the $g-2$ Geant 4 simulation with material effects and correlations included . . . . . . . . . 90

$4 \cdot 11$ Simulated true energy loss vs Geane predicted energy loss for tracks . $\quad 90$

$4 \cdot 12$ Track parameter truth pulls for fitted tracks in the $g-2$ Geant 4 simulation with material effects included . . . . . . . . . . . . . . . . . . 91

$4 \cdot 13$ Number of iterations for the track-fitting to converge . . . . . . . . . 92

$4 \cdot 14$ Number of planes hit for fitted tracks . . . . . . . . . . . . . . . . . . 92

$4 \cdot 15$ Fitted track momentum distribution and corresponding residuals to

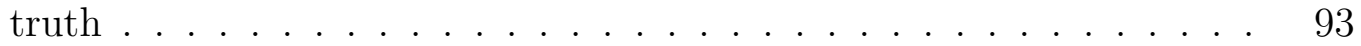

$4 \cdot 16$ Fitted track vertical and horizontal momenta and corresponding residuals to truth . . . . . . . . . . . . . . . . . . . . . . . . . 994

$4 \cdot 17$ Fitted track vertical and horizontal positions and corresponding residuals to truth . . . . . . . . . . . . . . . . . . 95

$4 \cdot 18$ Sample fitted track and p-value distribution for fitted tracks in data . 99

$4 \cdot 19$ Fitted tracks in data compared to Monte-Carlo, track times and total momentum . . . . . . . . . . . . . . . . . . . . 99

$4 \cdot 20$ Fitted tracks in data compared to Monte-Carlo, radial and vertical momentum and position distributions . . . . . . . . . . . . . . . . 100

$4 \cdot 21$ Track extrapolation schematic . . . . . . . . . . . . . . . . . . 102

$4 \cdot 22$ Bird's eye view of extrapolation in ring . . . . . . . . . . . . . . . . . 102

$4 \cdot 23$ Extrapolated muon beam distribution cross-section . . . . . . . . . . 105

$4 \cdot 24$ Muon beam radial and vertical distributions . . . . . . . . . . . . . . 105 
4.25 Muon beam radial and vertical distributions as a function of time in-fill 106

4.26 Muon beam radial and vertical distribution means as a function of time

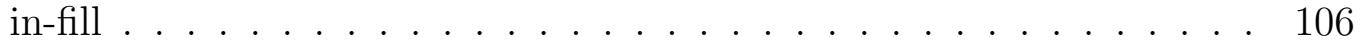

$4 \cdot 27$ Beam average vertical position and width as a function of time in-fill 107

$4 \cdot 28$ CBO frequency and amplitude as a function of time in-fill $\ldots \ldots$.

$5 \cdot 1$ Template fit to SiPM trace . . . . . . . . . . . . . . . . . . 111

$5 \cdot 2$ In-fill gain function fit for a single calorimeter crystal . . . . . . . . 113

$5 \cdot 3 \quad$ Calorimeter cluster from SiPM traces fit with templates. . . . . . . . 114

5.4 Sample energy spectrum . . . . . . . . . . . . . . . . 116

5.5 Determination of optimal energy threshold $\ldots \ldots \ldots \ldots \ldots$

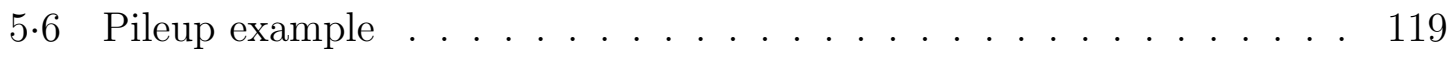

$5 \cdot 7 \quad$ Shadow pileup method $\ldots \ldots \ldots \ldots$

$5 \cdot 8$ Pileup time spectrum above threshold $\ldots \ldots \ldots \ldots \ldots$. . . . . . . 122

$5.9 \quad$ Cluster energies vs pileup energies . . . . . . . . . . . . . . 123

$5 \cdot 10$ Cluster energies divided by pileup energies . . . . . . . . . . . . 124

$5 \cdot 11$ Cluster times above $3.5 \mathrm{GeV}$. . . . . . . . . . . . . . . 125

$5 \cdot 12$ Non-corrected and pileup corrected cluster energies . . . . . . . 126

5.13 Ratio formation functions . . . . . . . . . . . . . . . . . . 129

$5 \cdot 14$ Cancellation of effect in Ratio Method versus frequency . . . . . . . . 130

$5 \cdot 15$ FFT of five parameter fit residuals $\ldots \ldots \ldots \ldots \ldots$

$5 \cdot 16$ FFT of three parameter ratio fit residuals $\ldots \ldots \ldots \ldots$

$5 \cdot 17$ VW amplitude resonance in the $60 \mathrm{~h}$ dataset $\ldots \ldots \ldots$. . . . 137

$5 \cdot 18$ VW envelope in Ratio Method Toy MC data with and without FR effect 138

$5 \cdot 19$ Lost muon $\Delta t$ and energy deposition distributions $\ldots \ldots \ldots \ldots$. . . 141

$5 \cdot 20$ Lost muon $\Delta t_{12}$ distribution as a function of time in-fill . . . . . . . 143

$5 \cdot 21$ Deuteron population at late times . . . . . . . . . . . . . . 144 
$5 \cdot 22$ Final $\Delta t_{12}$ distribution for selected lost muons . . . . . . . . . . . . . 145

$5 \cdot 23$ Lost muon triples spectrum compared to quadruples and accidental background . . . . . . . . . . . . . . . . . . . . . 145

$5 \cdot 24$ Single random seed fits to calorimeter summed data of Run 1 precession frequency analysis datasets . . . . . . . . . . . . . . . . . . . . . 150

$5 \cdot 25$ Pulls and FFT of residuals for the ratio fit to the 60h dataset . . . . 152

$5 \cdot 26 \chi^{2} /$ NDF's versus calorimeter number . . . . . . . . . . . . . 155

$5 \cdot 27 R$ versus calorimeter number . . . . . . . . . . . . . . . . 155

$5 \cdot 28$ Endgame fit parameters versus calorimeter number . . . . . . . . 156

$5 \cdot 29$ Additional Endgame fit parameters versus calorimeter number . . . . 157

$5 \cdot 30$ Examples of fit start scans when effects are improperly accounted for

before or during fitting . . . . . . . . . . . . . . . . . . . . . 158

$5 \cdot 31 \chi^{2} / \mathrm{NDF}$ and $R$ versus fit start time . . . . . . . . . . . . . 160

$5 \cdot 32$ Fit start scans for free parameters in the Endgame dataset . . . . . . 161

$5.33 R$ versus fit end time . . . . . . . . . . . . . . . . . . . 162

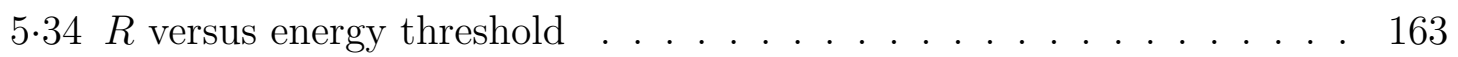

$5.35 R$ versus bunch number . . . . . . . . . . . . . . . . . . . . . 164

$5 \cdot 36 \quad \chi^{2}$ s and $R$ values for fits to many random seeds . . . . . . . . . . 166

$5 \cdot 37$ Pileup shadow dead time scan without automatic pileup amplitude scaling . . . . . . . . . . . . . . . . . . . . 170

$5 \cdot 38$ Pileup shadow dead time scan with automatic pileup amplitude scaling 171

$5 \cdot 39$ Pileup shadow gap time scan with automatic pileup amplitude scaling 172

5.40 Pileup multiplier scan . . . . . . . . . . . . . . . . . . . . . . . . . . . 174

5.41 Pileup time-shift scan . . . . . . . . . . . . . . . . . 175

5.42 Pileup energy scale scan . . . . . . . . . . . . . . . 177

5.43 IFG amplitude and lifetime scans . . . . . . . . . . . . . . . . . . 180 
$5 \cdot 44$ Lost muon $\Delta t_{13}$ distribution as a function of time in-fill . . . . . . . . 190

$5 \cdot 45$ Scan over fixed $\kappa_{\text {loss }}$ in ratio fit . . . . . . . . . . . . . . . . . . 191

5.46 Fractional muon losses in the Run 1 datasets . . . . . . . . . . . . . . 194

5.47 Scans over ratio construction parameters . . . . . . . . . . . . . . 196

5.48 Scans over binning parameters . . . . . . . . . . . . . . . . . . . . . . 198

5.49 Comparison between dataset mean $R$ values . . . . . . . . . . . . . . 205

A.1 Angular correction for measured DCAs . . . . . . . . . . . . . . . . 211

D.1 60h ratio fit correlation matrix . . . . . . . . . . . . . . . . 226

D.2 HighKick ratio fit correlation matrix . . . . . . . . . . . . . . . . . . 227

D.3 9d ratio fit correlation matrix . . . . . . . . . . . . . . . . . . . . . . 228

D.4 Endgame ratio fit correlation matrix . . . . . . . . . . . . . . . . 229

E·1 Mean $R$ value comparison in Toy MC between T-Method and Ratio Method ......................... 232 


\section{List of Abbreviations}

\begin{tabular}{|c|c|c|}
\hline $\mathrm{ADT}$ & .. & artificial dead time \\
\hline ASDQ & $\ldots$ & Amplifier Shaper Discriminator with charge $(\mathrm{Q})$ \\
\hline ASIC & & application specific integrated circuit \\
\hline BNL & & Brookhaven National Laboratory \\
\hline BSM & & beyond the standard model \\
\hline $\mathrm{CBO}$ & & coherent betatron oscillation \\
\hline E821 & & Brookhaven Muon $g-2$ Experiment \\
\hline E989 & & Fermilab Muon $g-2$ Experiment \\
\hline EW & & electroweak \\
\hline FID & .. & free-induction decay \\
\hline FNAL & & Fermi National Accelerator Laboratory \\
\hline FR & & fast rotation \\
\hline Geane & .. & Geometry and Error Propagation \\
\hline Geant & & Geometry and Tracking \\
\hline Had & .. & Hadronic \\
\hline HLbL & & hadronic light-by-light \\
\hline HVP & & hadronic vacuum polarization \\
\hline IBMS & .. & inflector beam monitoring system \\
\hline LO & & leading order \\
\hline MIP & & minimum-ionizing particle \\
\hline NLO & ... & next-to-leading order \\
\hline NMR & & nuclear magnetic resonance \\
\hline PCB & & printed circuitry board \\
\hline PMT & $\ldots$ & photo-multiplier tube \\
\hline POT & $\ldots .$. & protons on target \\
\hline $\mathrm{ppb}$ & & parts per billion \\
\hline ppm & n......... & parts per million \\
\hline ppt & .. & parts per trillion \\
\hline QCD & $\ldots$ & quantum chromodynamics \\
\hline QED & & quantum electrodynamics \\
\hline RMS & & root mean square \\
\hline SAM & $\ldots \ldots \ldots \ldots$ & Sequential Access via Metadata \\
\hline SDT & & shadow dead time \\
\hline SGT & $\ldots \ldots \ldots \ldots$ & shadow gap time \\
\hline
\end{tabular}




$\begin{array}{lll}\text { SiPM } & \ldots \ldots \ldots \ldots & \text { silicon photo-multiplier } \\ \text { SM } & \ldots \ldots \ldots \ldots & \text { Standard Model } \\ \text { STDP } & \ldots \ldots \ldots \ldots & \text { short-term double pulse } \\ \text { VW } & \ldots \ldots \ldots \ldots & \text { vertical waist } \\ \text { WFD } & \ldots \ldots \ldots \ldots & \text { waveform digitizer }\end{array}$




\section{Chapter 1}

\section{Introduction}

The prevailing theory of particle physics, the Standard Model (SM), has had tremen-

dous success in describing our universe. It has been used to predict and explain a wide variety of phenomena, particle properties, and interactions to great precision. However, in spite of its success in explaining nearly all experimental results, there remain unanswered questions about our universe. Some of these include the matterantimatter asymmetry, the source of mass for the neutrinos, the existence of dark matter, and reconciling general relativity and quantum mechanics. Many particle physics experiments around the world are being devised and conducted in order to shed light on these questions and improve our understanding of reality. In general, particle physics experiments search for new physics by looking directly for new particles or processes, forbidden or otherwise, or by precisely measuring quantities that are predicted by the SM in order to search for deviations between theory and experiment. One such experiment in the latter case is the Fermilab Muon $g-2$ Experiment (E989), underway at the Fermi National Accelerator Laboratory located in Batavia, Illinois.

This dissertation will describe the E989 experiment and the author's contributions in detail. Chapter 1 will provide experimental and theoretical background to the experiment, as well its motivation. Chapter 2 will describe the experimental principle and specifics of muon production and storage. Chapter 3 will describe the various detector systems. Chapter 4 will describe the straw tracking reconstruction including 
the track fitting algorithm, as well as some analysis results. Chapter 5 will describe the precession frequency measurement portion of the experiment, and detail analysis results from data taken in the first run of the experiment in 2018. Chapter 6 will conclude the dissertation and the results contained within.

\subsection{Magnetic moments of particles}

All particles have intrinsic properties. One property of charged particles is the magnetic dipole moment.1 This property of a particle is related to its spin through the equation

$$
\vec{\mu}=g \frac{q}{2 m} \vec{s}
$$

where $\vec{\mu}$ is the magnetic dipole moment of a particle, $\vec{s}$ is its spin vector, $m$ is its mass, $q= \pm e$ where $e$ is the elementary charge, and $g$ is the so called "Landé g-factor". $g$ is a measurable and predictable observable. Since the torque on a particle in a magnetic field $\vec{B}$ is

$$
\vec{N}=\vec{\mu} \times \vec{B}
$$

the rate at which a particle's spin precesses in a magnetic field will depend on $g$. This is one of the key physics principles in the E989 experiment as will be discussed later.

In a Dirac theory, $g$ is equal to 2 for spin- $\frac{1}{2}$ particles with no internal structure [1]. A simple derivation of this result is given in Reference [2]. However, even for these types of particles, $g$ is not quite equal to 2 . Motivated by early experimental measurement discrepancies such as the measurements of the hyperfine structure in hydrogen [3], in 1948 Schwinger calculated the first "radiative correction" to the electron magnetic moment [4]. In the same year Kusch and Foley measured the $g$ factor of the

\footnotetext{
${ }^{1}$ Magnetic dipole moment and magnetic moment are equivalent when talking about particles.
} 
electron to be $g_{e}=2.00238 \pm 0.00010$ [5, 6], proving that such radiative corrections were needed in a properly descriptive theory. In a quantum field theory, interactions of the particle with virtual particles in loops will contribute to the value of $g$. In this context, for charged leptons, it is convenient to recast the magnetic dipole moment formula as

$$
\begin{aligned}
\vec{\mu} & =2(1+a) \frac{q}{2 m} \vec{s}, \\
a & =\frac{g-2}{2},
\end{aligned}
$$

where $a$ is called the "magnetic anomaly," and contains all higher order corrections to $g$. The first correction to $a$, calculated by Schwinger, was $a=\alpha / 2 \pi \approx 0.00116$, where $\alpha$ is the fine structure constant, consistent with the measured value. By measuring $a$, the SM theory can be tested and extensions to it constrained. A precise measurement of the muon magnetic anomaly, or the anomalous magnetic moment of the muon, is the main goal of the Fermilab Muon $g-2$ Experiment.

\subsection{Standard Model contributions to $a_{\mu}$}

The latest theoretical predictions for the muon magnetic moment will be presented here. The contributions to $a_{\mu}$ can be divided into three sectors of the SM. These include the quantum-electrodynamics (QED) contributions purely from leptons and photons, the electroweak $(\mathrm{EW})$ contributions from interactions involving the weak

force bosons $\left\{W^{ \pm}, Z^{0}, H\right\}$, and the hadronic (Had) contributions from interactions with strongly-interacting hadrons:

$$
a_{\mu}^{\mathrm{SM}}=a_{\mu}^{\mathrm{QED}}+a_{\mu}^{\mathrm{EW}}+a_{\mu}^{\mathrm{Had}}
$$




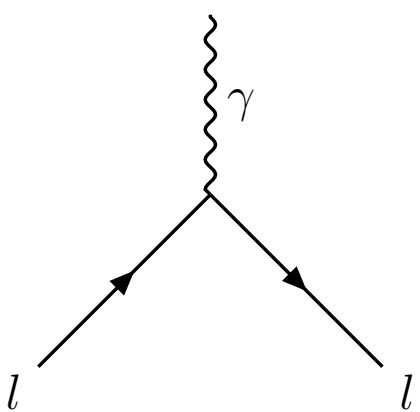

(a) Dirac result, $g=2$.

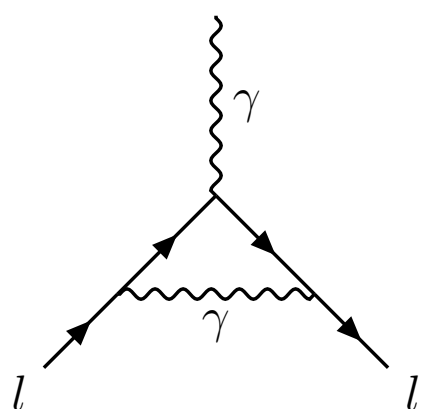

(b) The first loop diagram, calculated by Schwinger.

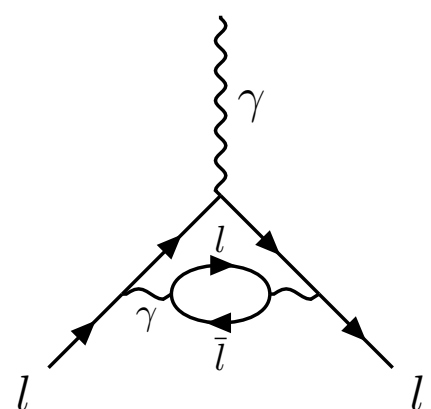

(c) A two-loop diagram.

Figure 1.1: The first of many QED diagrams contributing to $a$. Feynman diagrams made using TikZ-FeynHand [7, 8].

\subsubsection{QED}

The QED contributions to $a_{\mu}$ stem solely from loops with virtual leptons and photons. They are well understood and have been calculated to sufficient order, having been calculated up to five-loop level from over 13,000 Feynman diagrams [9, 10]. This has been achieved through both analytical and numerical methods. The first couple of diagrams, including the Dirac $g=2$ and Schwinger diagrams, are shown in Figure $1 \cdot 1$. The sum of QED contributions to five-loop level calculated by Aoyama et al. is 9 , 10

$$
\begin{aligned}
a_{\mu}^{\mathrm{QED}} & =\sum_{n=1}^{\infty} C_{n}\left(\frac{\alpha}{\pi}\right)^{n}, \\
& =(11658471.8971 \pm 0.0007) \times 10^{-10},
\end{aligned}
$$

where in the first line $a_{\mu}^{\mathrm{QED}}$ is expressed as a perturbative expansion in the fine structure constant, $C_{n}$ is the coefficient to be determined, and $n$ is the loop level. $C_{1}=1 / 2$ is the Schwinger result mentioned previously, stemming from the diagram shown in Figure $1 \cdot 1 \mathrm{~b}$. While over $99 \%$ of the value of $a_{\mu}$ comes from the QED sector, the error is much smaller than in the EW and hadronic contributions. 


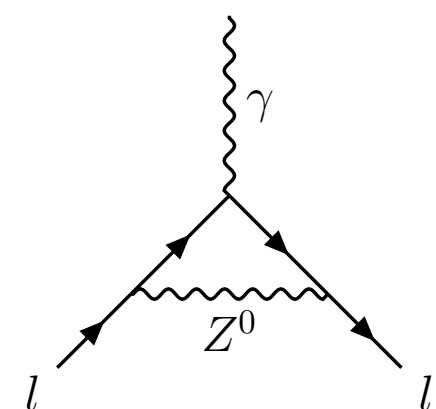

(a) Exchange of a virtual $Z^{0}$ boson.

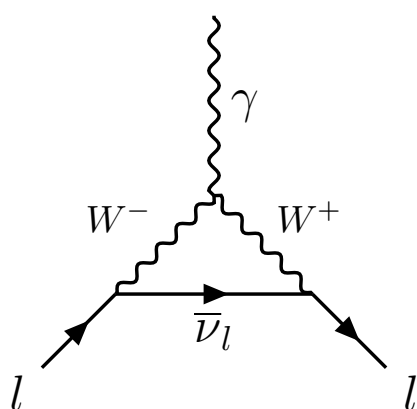

(b) Electroweak loop with $W$ bosons.

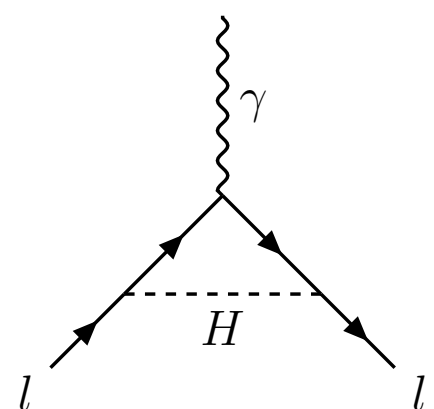

(c) Exchange of a Higgs boson.

Figure 1.2: First order weak diagrams contributing to $a$. Feynman diagrams made using TikZ-FeynHand [7, 8].

\subsubsection{Electroweak}

The electroweak contributions to $a_{\mu}$ are known to two-loop level [11]. Estimations of three-loop level contributions and above are small $[12]$. The different one-loop diagrams are shown in Figure $1 \cdot 2$. In the EW diagrams the heavy masses of the gauge bosons will produce contributions with characteristic scales of of $\sim\left(m_{l} / m_{Z^{0}, H, W^{ \pm}}\right)^{2}$. Because the masses of the gauge bosons are much larger than the muon, these processes are suppressed and the electroweak contributions to $a_{\mu}$ are small relative to the QED contributions. The value of the electroweak contributions as given by Ishikawa et al. is 11

$$
a_{\mu}^{\mathrm{EW}}=(15.29 \pm 0.10) \times 10^{-10} .
$$

This value is consistent with the oft-quoted PDG value of $15.36 \pm 0.10 \times 10^{-10}[12$. It should be noted that the error on these contributions is small compared to those of the hadronic contributions discussed next. 


\subsubsection{Hadronic}

The hadronic contributions to $a_{\mu}$ arise from loop diagrams with virtual hadrons. Because the strong coupling is large at low energies, the amplitudes for these processes cannot be calculated perturbatively. As the low-energy non-perturbative processes dominate the hadronic contributions, they by extension have errors that dominate the theoretical uncertainty in the SM calculation. Most active work on $a_{\mu}$ in the theoretical community is in this sector. The Muon $g-2$ Theory Initiative is comprised of many working groups from various institutions which seeks to make improvements in the hadronic calculations, and produce consensus values going forward in 2020 and beyond [13]. The hadronic contributions can be separated into two parts:

$$
a_{\mu}^{\mathrm{Had}}=a_{\mu}^{\mathrm{HVP}}+a_{\mu}^{\mathrm{HLbL}}
$$

\section{Hadronic Vacuum Polarization}

The first term in Equation 1.7 refers to contributions from hadronic vacuum polarization (HVP), the first order diagram of which is shown in Figure $1 \cdot 3 \mathrm{a}$. There are two main prescriptions for calculating these contributions. The first is to use a dispersive approach to introduce a virtual hadron bubble into the integral calculation for the photon propagator $2^{2}$, and then utilize the optical theorem to relate the imaginary part of that propagator to the total cross-section of electron-positron annihilation to hadrons [15]. While this could be solved perturbatively for a lepton bubble in place of the hadron bubble, this is instead a data driven approach when considering non-perturbative QCD. The leading order (LO) contribution can be written as

$$
a_{\mu}^{\mathrm{HVP} ; \mathrm{LO}}=\left(\frac{\alpha m_{\mu}}{3 \pi}\right)^{2} \int_{m_{\pi}^{2}}^{\infty} \frac{d s}{s^{2}} K(s) R(s)
$$

\footnotetext{
${ }^{2}$ The details of dispersion theory will not be described here but a pedagogical introduction is given in Reference 14.
} 


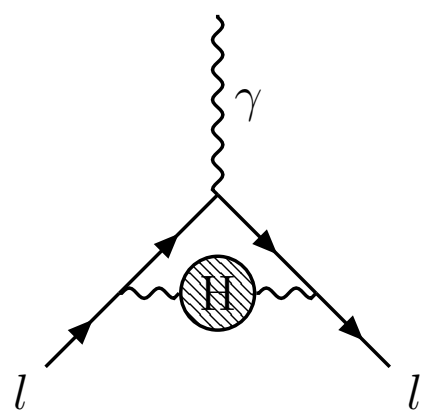

(a) The first order HVP Feynman diagram.

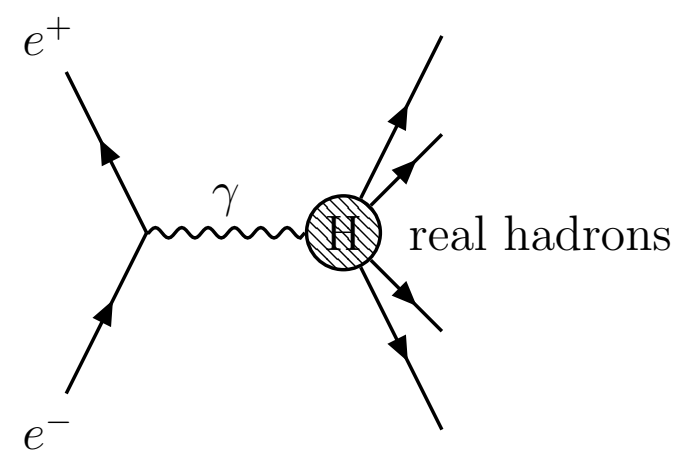

(b) The Feynman diagram for electron positron annihilation to hadrons.

Figure 1.3: The first order HVP diagram on the left, which can be related to the diagram on the right via the optical theorem. The $\mathrm{H}$ bubble in both diagrams indicates all hadrons. Feynman diagrams made using TikZ-FeynHand [7, 8].

where $K(s)$ is some calculable kinematic factor, and $R(s)$ is a ratio of cross-sections,

$$
R(s)=\frac{\sigma\left(e^{+} e^{-} \rightarrow \text { hadrons }\right)}{\sigma\left(e^{+} e^{-} \rightarrow \mu^{+} \mu^{-}\right)}
$$

The cross-section data for Equation 1.9 has been measured in different energy ranges by various experiments; a brief overview is given by A. Keshavarzi [16]. There are two approaches to measuring this cross-section data. The first operates at fixed center of mass energies in the standard collider theme, and the second tags initial state radiation in order to evaluate the differential cross-section over a wider energy range. As further data are acquired and compared between the different experiments, the error in the HVP contributions to $a_{\mu}$ will decrease. The analysis by Keshavarzi et al. with cross-section data as of November 1st, 2019 gives results as [17, 18

$$
\begin{aligned}
a_{\mu}^{\mathrm{HVP} ; \mathrm{LO}} & =(692.78 \pm 2.42) \times 10^{-10} \\
a_{\mu}^{\mathrm{HVP} ; \mathrm{NLO}} & =(-9.83 \pm 0.04) \times 10^{-10}
\end{aligned}
$$

where $a_{\mu}^{\mathrm{HVP} ; \mathrm{NLO}}$ is the next-to-leading (NLO) order calculation. This evaluation is 
consistent with another more conservative analysis by Davier et al. [19, 20]. A calculation for the next-to-next-to-leading order HVP contribution is given by Kurz et al. 21

$$
a_{\mu}^{\mathrm{HVP} ; \mathrm{NNLO}}=(1.24 \pm 0.01) \times 10^{-10}
$$

The second prescription for estimating the HVP contributions is a first principles approach, using lattice QCD. Lattice QFT is a gauge theory defined on a finite ensemble of discretized points in time and space. In the limit that the ensemble is taken infinitely large with the spacing between the points infinitesimally small, the behavior from a continuous theory is recovered. The lattice-based estimates of $a_{\mu}^{\mathrm{HVP} ; \mathrm{LO}}$ are consistent with those provided above, though the error is larger 22 .

\section{Hadronic Light-by-Light}

The second of these hadronic contribution parts is a higher-order, four-photon interaction, termed hadronic light-by-light (HLbL). Diagrams are shown in Figure 1.4. The calculation of these diagrams has historically been model dependent, and has therefore been the most contentious part of the SM calculation. The value of the HLbL contributions to $a_{\mu}$ from model estimates by the so-called 'Glasgow consensus' is $a_{\mu}^{\mathrm{HLbL}}=(10.5 \pm 2.6) \times 10^{-10}[23]$.

In more recent years, there have been efforts to produce results using dispersive 24 , 25, 26, 27, 28, 29, 30, 31] and lattice approaches [32, 33]. A calculation of the HLbL contribution using the sum of the dispersive results with linearly added errors given by Keshavarzi et al. from the references listed above is 18$]^{3}$

$$
a_{\mu}^{\mathrm{HLbL}}=(9.34 \pm 2.92) \times 10^{-10}
$$

This calculation, while consistent with the Glasgow consensus estimate provided

\footnotetext{
${ }^{3}$ Some pieces are still from the Glasgow consensus which are a work in progress. These dominate the error in the HLbL contribution.
} 

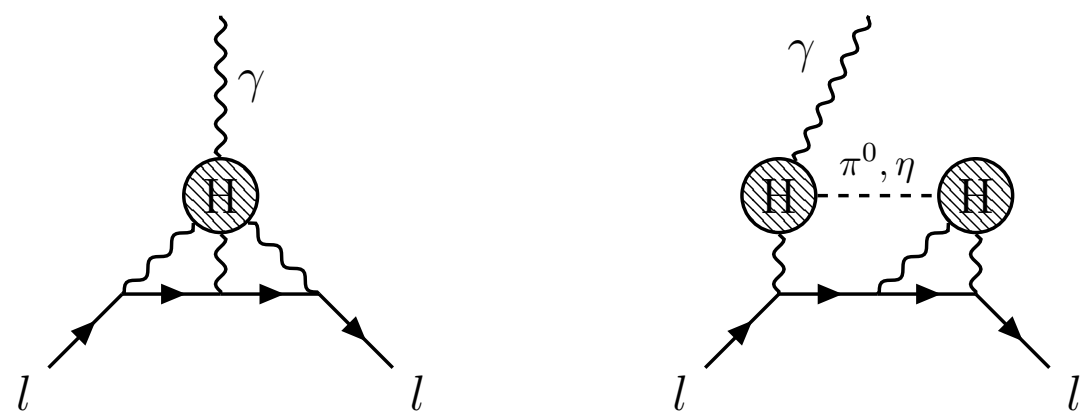

Figure 1.4: HLbL diagrams contributing to $a_{\mu}$, where three photons are exchanged with a virtual hadrons bubble. The diagram on the right is technically contained within the diagram on the left, however it is displayed separately here in order to help illustrate the complexity of this contribution. Feynman diagrams made using TikZ-FeynHand 7 , 8 .

above, has the advantage that it is in part model-independent. The error on this contribution is relatively large in each of these various approaches, comparable to that of the $a_{\mu}^{\mathrm{HVP} ; \mathrm{LO}}$ term, even though the size of this contribution is small. A calculation of the higher order HLbL contributions by Colangelo et al. gives [34

$$
a_{\mu}^{\mathrm{HLbL} ; \mathrm{NLO}}=(0.30 \pm 0.20) \times 10^{-10},
$$

showing that higher order HLbL contributions are practically negligible.

\subsubsection{Combined Standard Model value}

The sum of the $a_{\mu}$ contributions listed here is $[9,10,11,18,21,23,34$

$$
\begin{aligned}
a_{\mu}^{\mathrm{SM}} & =a_{\mu}^{\mathrm{QED}}+a_{\mu}^{\mathrm{EW}}+a_{\mu}^{\mathrm{Had}}, \\
& =(11659181.02 \pm 3.80) \times 10^{-10} .
\end{aligned}
$$

The relative uncertainty of this result is 326 parts per billion (ppb). Other analyses with different values for the various contributions typically agree well, as shown on the left side of Figure 1.5. In general, the consistency of the theory has been stable 


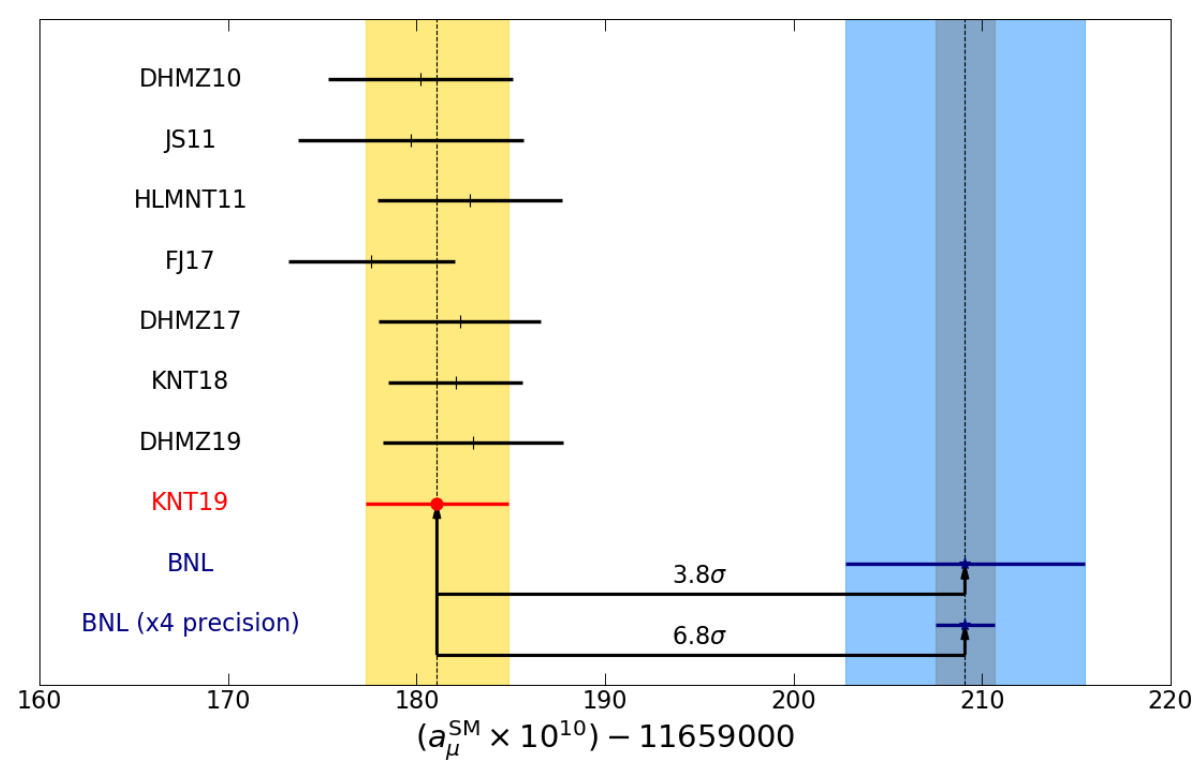

Figure 1.5: Various theoretical values for $a_{\mu}$ on the left, as compared to the most recent and future extrapolated experimental results on the right. From Reference [18].

for almost ten years now. Depending on which calculations are used, the discrepancy between theory and experiment ranges between 3 to 4 standard deviations.

\subsection{Experimental value of $a_{\mu}$ and discrepancy with $a_{\mu}^{\mathrm{SM}}$}

The theoretical contributions to $a_{\mu}$ listed in the previous sections have improved over time as methods have matured and more experimental data have been gathered. Similarly, work on the direct experimental measurement of $a_{\mu}$ has been going on for decades, with more precise results being determined over time [35]. The most recent experiment to measure $g-2$ was the Brookhaven Muon $g-2$ Experiment (E821) held at Brookhaven National Laboratory (BNL), which collected data from 1997 to 2001. That experiment measured a value for $a_{\mu}$ of 36, 37]

$$
a_{\mu}^{\text {Exp }}=(11659208.9 \pm 6.3) \times 10^{-10}
$$


which corresponds to a $540 \mathrm{ppb}$ relative uncertainty. Note that the uncertainty of the experimental measurement is comparable to that of the theory. The difference between the experimental and theoretical values presented here is

$$
a_{\mu}^{\mathrm{Exp}}-a_{\mu}^{\mathrm{SM}}=(27.88 \pm 7.36) \times 10^{-10},
$$

corresponding to a discrepancy of 3.79 standard deviations.

\subsection{Beyond the Standard Model and the purpose of E989}

While the discrepancy between experiment and theory might be attributed to miscalculations in the theory or systematic errors in the E821 experiment, or a very rare statistical fluctuation, no such errors have been found despite repeated attempts to resolve it. Indeed the discrepancy has only grown over time as the theoretical calculations have matured. The most intriguing and exciting source of the discrepancy would be physics beyond the standard model (BSM).

The value of $a_{\mu}$ receives contributions from all particles that couple to the muon through virtual loops, so it is possible that as yet undiscovered particles are the source of this discrepancy. The contribution to the magnetic moment from heavy virtual particles goes as

$$
a \sim C \frac{m^{2}}{\Lambda^{2}}
$$

where $C$ is a constant of $\mathcal{O}(1), \Lambda$ is the mass scale of the new physics, and $m$ the mass of the lepton in question. The postulation of such new heavy particles has long been explored in supersymmetry models [38]. With the lack of new physics results from the LHC experiments however, such models have increasingly been excluded. In spite of this, there are still regions of the parameter space that could account for the $g-2$ discrepancy [39]. Radiative mass mechanisms in the range of $1-2 \mathrm{TeV}$ can also be 
the source of the discrepancy while also accounting for the small muon mass relative to the electroweak gauge bosons [38]. Heavy scalar leptoquarks, two-Higgs-doublet, or more general scalar doublet models can also be constructed which account for the discrepancy $40,41,42]$.

The sensitivity of the muon as compared to the electron to large mass scales is $m_{\mu}^{2} / m_{e}^{2} \approx 43,000$ times greater. For this reason, it is possible that even though the magnetic anomaly of the electron $a_{e}$ has been measured extraordinarily precisely, to $0.23 \mathrm{ppb}$ corresponding to a 0.26 parts per trillion (ppt) relative uncertainty in the electron g-factor [37, 43], it has not yielded a definitive difference between theory and experiment, whereas the magnetic moment of the muon might do so. With that being said a recent measurement of the fine structure constant $\alpha$ to the highest precision yet [44] has yielded a new value of $a_{e}$ for which the difference between theory and experiment is 2.5 standard deviations, but with the opposite sign as compared to $a_{\mu}$. To explain both discrepancies simultaneously implies that the electron and muon sectors need to be decoupled from one another in light of the constraints from $\mu \rightarrow e \gamma$. Models with vector-like fermions which can account for both discrepancies can be formulated whereas other models have trouble [45].

BSM models at the low energy regime can also explain the discrepancy. These include a light scalar 'dark' Higgs which may be capable of producing the discrepancy [46], (regardless of the fact that the dark photon model has been ruled out at the $99 \%$ confidence level 47$]$ ), and low mass pseudo-scalar axion-like particles [48], among others.

Future data taken from the LHC and other experiments will further constrain the possible models based on other experimental observables. The Fermilab E989 experiment was undertaken in order to improve the experimental precision of the $a_{\mu}$ measurement, while verifying the previous result. Although, in general, there has 


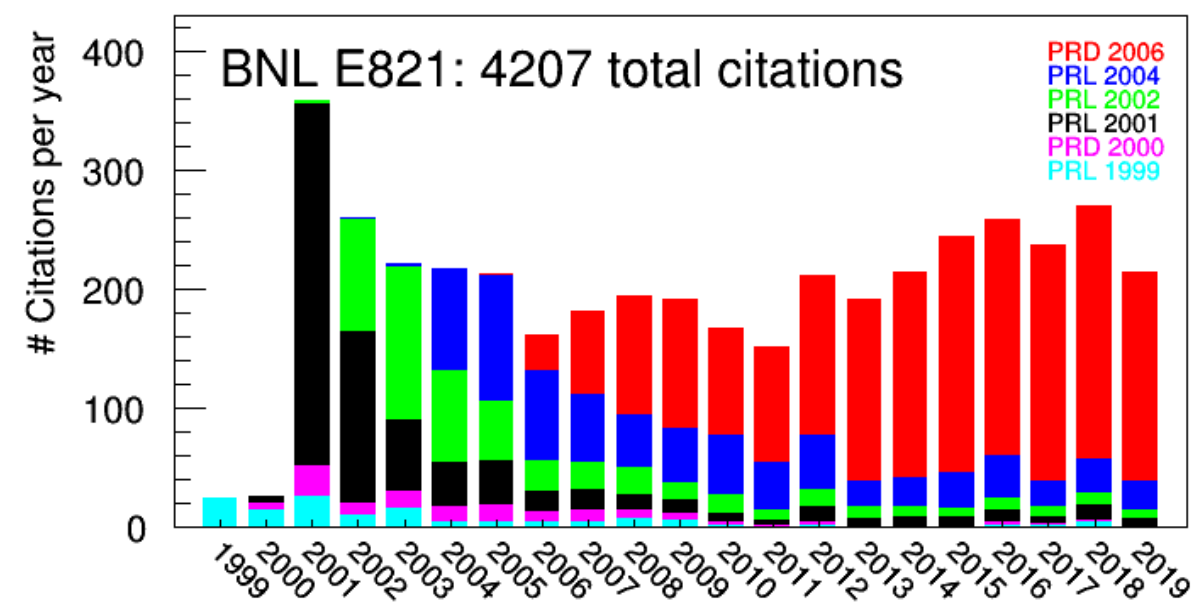

Figure 1.6: The number of citations for the BNL experiment E821 publications as a function of year, as of 10/30/2019 [49].

been a lack of new physics results from other experiments, E989 has the potential to provide an indirect confirmation of new physics. Accordingly, interest in the E821 experiment and it's successor E989 has grown over time. The number of citations for the E821 results has been consistently high for 20 years as shown in Figure 1.6.

The E989 experiment has the goal of measuring $a_{\mu}$ to $140 \mathrm{ppb}$ by 2021 . This would be a factor of four improvement over the E821 result stemming from a twenty times increase in statistics, which was the dominant error in the previous experiment. Assuming the same central value for $a_{\mu}$ is obtained, this new measurement would push the statistical significance of the discrepancy to approximately seven standard deviations, as shown in Figure 1.5. The data comprising Run 1, gathered between April and July of 2018, is the subject of this thesis, and corresponds to a statistical uncertainty comparable to the E821 result. The Run 1 result by itself may provide strong evidence for new physics, depending on the new measured value for $a_{\mu}$. 


\section{Chapter 2}

\section{Principal Techniques of E989}

A particle with non-zero spin in a magnetic field will experience a torque which attempts to line up the magnetic dipole moment of the particle with the external field. As a result a particle's spin in a dipole field will turn at the spin precession frequency 50

$$
\vec{\omega}_{s}=-g \frac{q}{2 m} \vec{B}-(1-\gamma) \frac{q}{\gamma m} \vec{B}
$$

where $m$ is the particle's mass, $q= \pm e$ where $e$ is the positive elementary charge, $g$ is the g-factor, $\gamma$ is the Lorentz relativistic factor, and $B$ is an external magnetic field. The first term is the usual Larmor frequency and the second term is a relativistic correction to the precession frequency in an accelerating frame called Thomas preces-

sion. Similarly, a particle with some momentum perpendicular to the magnetic field will orbit at the cyclotron frequency

$$
\vec{\omega}_{c}=-\frac{q}{\gamma m} \vec{B}
$$

By taking the difference between these two frequencies we arrive at the "spin difference frequency,"

$$
\vec{\omega}_{a}=\vec{\omega}_{s}-\vec{\omega}_{c}=-\frac{g-2}{2} \frac{q}{m} \vec{B}=-a \frac{q}{m} \vec{B},
$$


a frequency that is directly proportional to the anomaly $a$. If $g=2$, as in a Dirac theory, then the particle's spin would turn at the same rate as the momentum vector, and $\omega_{a}$ would be identically zero. If $\omega_{a}$ for a muon and the external magnetic dipole field can be measured, then the anomalous magnetic moment of the muon $a_{\mu}$ can be determined.

As will be detailed in Section 2.2, the measurement of the magnetic field is related to the Larmor precession frequency of free protons in water

$$
\omega_{p}=-g_{p} \frac{e}{2 m_{p}} B
$$

where $g_{p}$ and $m_{p}$ are the g-factor and mass of the proton respectively. Replacing $B$ and solving for $a_{\mu}$, we arrive at

$$
a_{\mu}=\frac{g_{p}}{2} \frac{\omega_{a}}{\omega_{p}} \frac{m_{\mu}}{m_{p}}
$$

Using the magnetic moment formulae for the proton, electron, and muon as shown in Equation 1.1, Equation 2.5 can be transformed into

$$
a_{\mu}=\frac{g_{e}}{2} \frac{\omega_{a}}{\omega_{p}} \frac{m_{\mu}}{m_{e}} \frac{\mu_{p}}{\mu_{e}}
$$

where the $p, e$, and $\mu$ subscripts stand for the relevant quantities for the proton, electron, and muon respectively. The experimental error on $a_{\mu}$ then becomes the quadrature sum of each individual quantity error. As mentioned in Section 1.4 the electron g-factor $g_{e}$ has been measured to extremely high precision, $0.26 \mathrm{ppt}$ 37, 43. The muon-electron mass ratio, $m_{\mu} / m_{e}$, has been measured to $22 \mathrm{ppb}$ [37, 51]. Finally the proton-electron magnetic moment ratio, $\mu_{p} / \mu_{e}$, has been measured to $3 \mathrm{ppb} 37$. These are small compared to the target statistical error on $\omega_{a}$ of $100 \mathrm{ppb}$, and target systematic errors on $\omega_{a}$ and $\omega_{p}$, both at $70 \mathrm{ppb}^{1}$. These errors added in quadrature

\footnotetext{
${ }^{1}$ The measurement of $\omega_{p}$ has negligible statistical error.
} 
is approximately $140 \mathrm{ppb}$, which is the target of the E989 experiment.

\section{$2.1 \quad$ Measuring $\omega_{a}$}

How can $\omega_{a}$ for muons be measured? The answer lies with two key points in the dynamics of muon decay. Positive muons decay to a positron and two neutrinos, as shown in Figure 2.1. The first point is that because of the parity violating nature of the weak interaction, the decay positron will preferentially be emitted right-handed, with its spin directed in the same direction as its momentum [52]. The second key point is that angular momentum must be conserved. Consider the most extreme examples of maximum and minimum energy decay positrons as shown in Figure 2.2. In the muon rest frame, decay positrons with maximum energy will be emitted opposite to the two neutrinos, both emitted in the same direction. Since neutrinos and anti-neutrinos must be left and right-handed respectively, with their spins directed anti-parallel and parallel to their momentum, by the law of conservation of angular momentum the positron spin must be parallel to the spin of the muon at the time of the decay. By the opposite argument, decay positrons emitted with minimum energy such that the neutrinos are ejected opposite to one another must have their spins be anti-parallel to that of the muon at the time of decay. Together, these two points mean that higher energy decay positrons will preferentially be emitted in directions parallel to the muon spin at the time of decay, while lower energy decay positrons will preferentially be emitted in directions anti-parallel to the muon spin at the time of the decay.

This correlation between the direction of an emitted high energy decay positron and the spin of the muon is the signature needed to measure $\omega_{a}$. Muons placed within a magnetic storage ring will orbit at the cyclotron frequency and their spins will precess at the spin precession frequency. As they go around the ring they will decay to positrons whose energy and decay directions contain information about the 


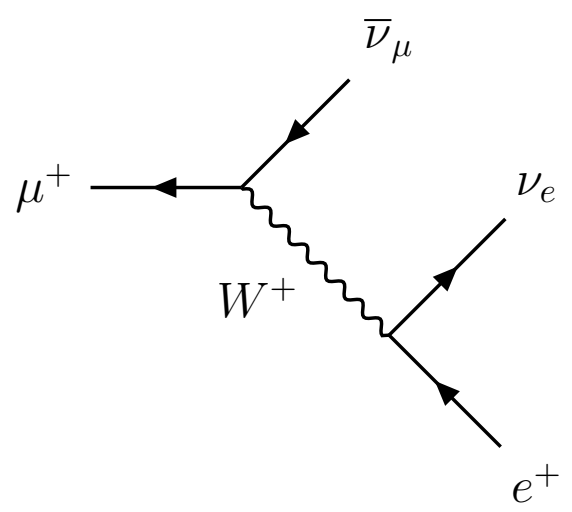

Figure 2.1: Diagram for muon decay. $\mu^{+}$decay through a $W^{+}$boson to a positron, muon anti-neutrino, and an electron neutrino. This process consists of nearly $100 \%$ of the muon decay branching ratio, with other decay states including radiative processes.

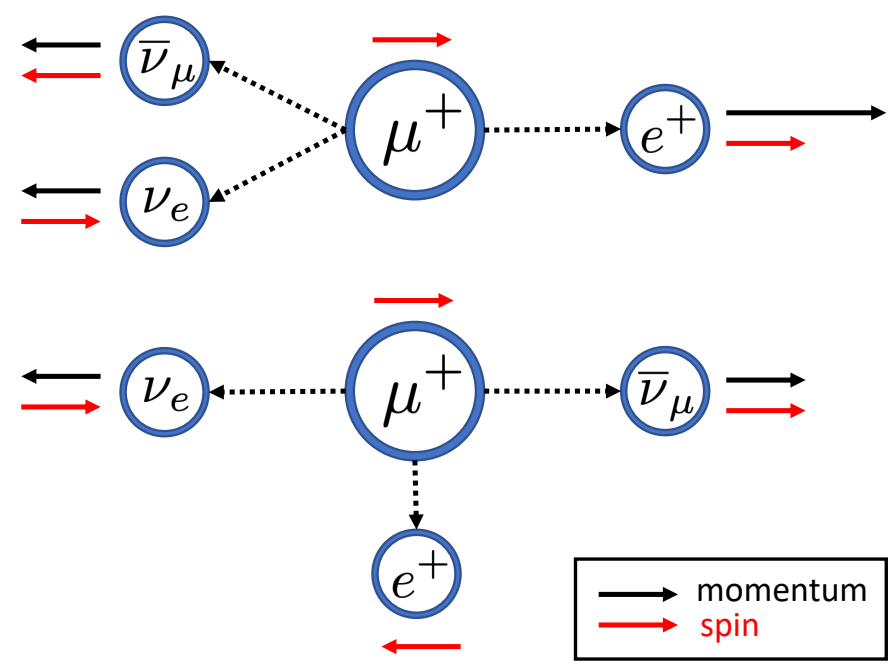

Figure 2.2: Muon decay in the rest frame for maximum (top) and minimum (bottom) energy decay positrons. Due to the conservation of angular momentum and the single possible helicity states of the decay neutrinos, the spin of the decay positron is exactly parallel to the spin of the muon at the time of the decay for maximum energy decay positrons, or anti-parallel for minimum energy decay positrons. 
spin of the muons. If the muon ensemble is un-polarized, then the decay distribution for all positrons will be isotropic and in general un-useful. If the muon ensemble is polarized, then the decay distribution for each muon will be the same, and allow for a measurement of $\omega_{a}$, as described in the following text.

The differential decay distribution for positive muons in the muon rest frame is described by 52

$$
d P(y, \theta) \propto N(y)[1+A(y) \cos (\theta)] d y d \Omega
$$

where $y=E / E_{\max }$ is the energy fraction of the positron and $\theta$ is the angle between the spin of the muon and the momentum of the positron at the time of decay. $N(y)$ is the number distribution of decay positrons and $A(y)$ is the so called 'asymmetry,' encoding the energy-dependent correlation between the muon spin and the decay positron direction. Here the energy of the positron is assumed to be much greater than its mass. The number distribution and asymmetry are given by

$$
\begin{aligned}
& N(y)=2 y^{2}(3-2 y), \\
& A(y)=\frac{2 y-1}{3-2 y},
\end{aligned}
$$

and are shown in Figure 2·3a.

In the lab frame, nearly all high energy positrons are emitted parallel to the muon momentum, making it challenging to select purely on the decay angle of the positron. That is not a problem however, as we already know that decay positrons with the highest rest-frame energies will be emitted parallel to the muon spin at the time of decay. Essentially, the energy distribution of detected high energy positrons is modulated by $\omega_{a}$, or $\theta=\omega_{a} t+\phi$. The number of detected positrons at some time and energy in the lab frame for some initial number $N_{0}$ of muons can then be described 


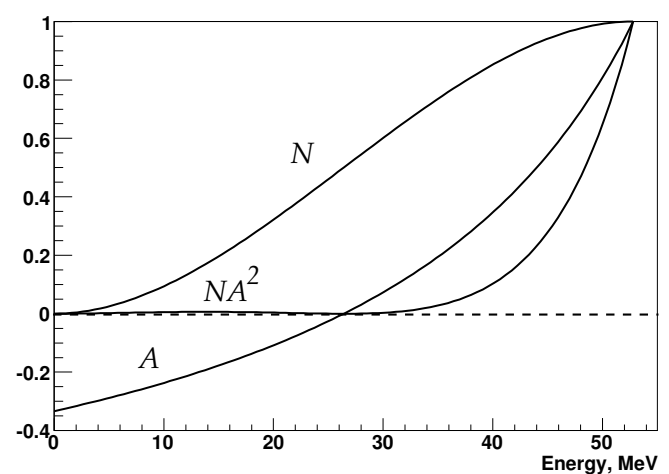

(a) Muon rest frame

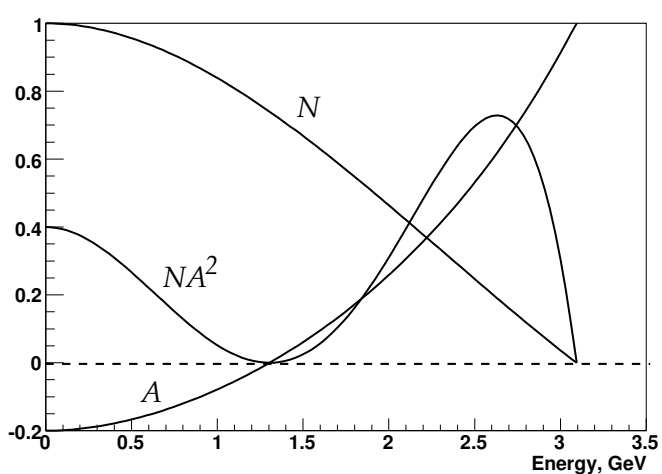

(b) Lab frame

Figure 2.3: Decay number distribution $N$ and asymmetry $A$ in the muon rest frame (left) and in the lab frame (right) as a function of positron energy with a maximum positron energy of $3.1 \mathrm{GeV} . \quad N$ is multiplied by arbitrary factors in both pictures.

by

$$
N_{d}(t, E)=N_{0}(E) \cdot e^{-t / \gamma \tau_{\mu}} \cdot\left[1+A(E) \cos \left(\omega_{a} t+\phi(E)\right)\right]
$$

where the $d$ subscript stands for 'detected,' the muons are decaying with a lifetime of $\gamma \tau_{\mu}$, and all the relevant parameters are energy dependent. Here $N_{0}(E)$ and $A(E)$ have been transformed from Equations 2.8 and 2.9 to the lab frame:

$$
\begin{aligned}
N_{0}(E) & \propto(y-1)\left(4 y^{2}-5 y-5\right) \\
A(E) & =\frac{-8 y^{2}+y+1}{4 y^{2}-5 y-5}
\end{aligned}
$$

Here the polarization of the muons is assumed to be unity. These relations are shown in Figure $2 \cdot 3 \mathrm{~b}$, All positrons above some energy threshold cut $E_{t h}$ can be taken as the observable,

$$
N_{d}\left(t, E_{t h}\right)=N_{0}\left(E_{t h}\right) \cdot e^{-t / \gamma \tau_{\mu}} \cdot\left[1+A\left(E_{t h}\right) \cos \left(\omega_{a} t+\phi\left(E_{t h}\right)\right)\right]
$$

where the number and asymmetry of the detected positrons is now calculated by 


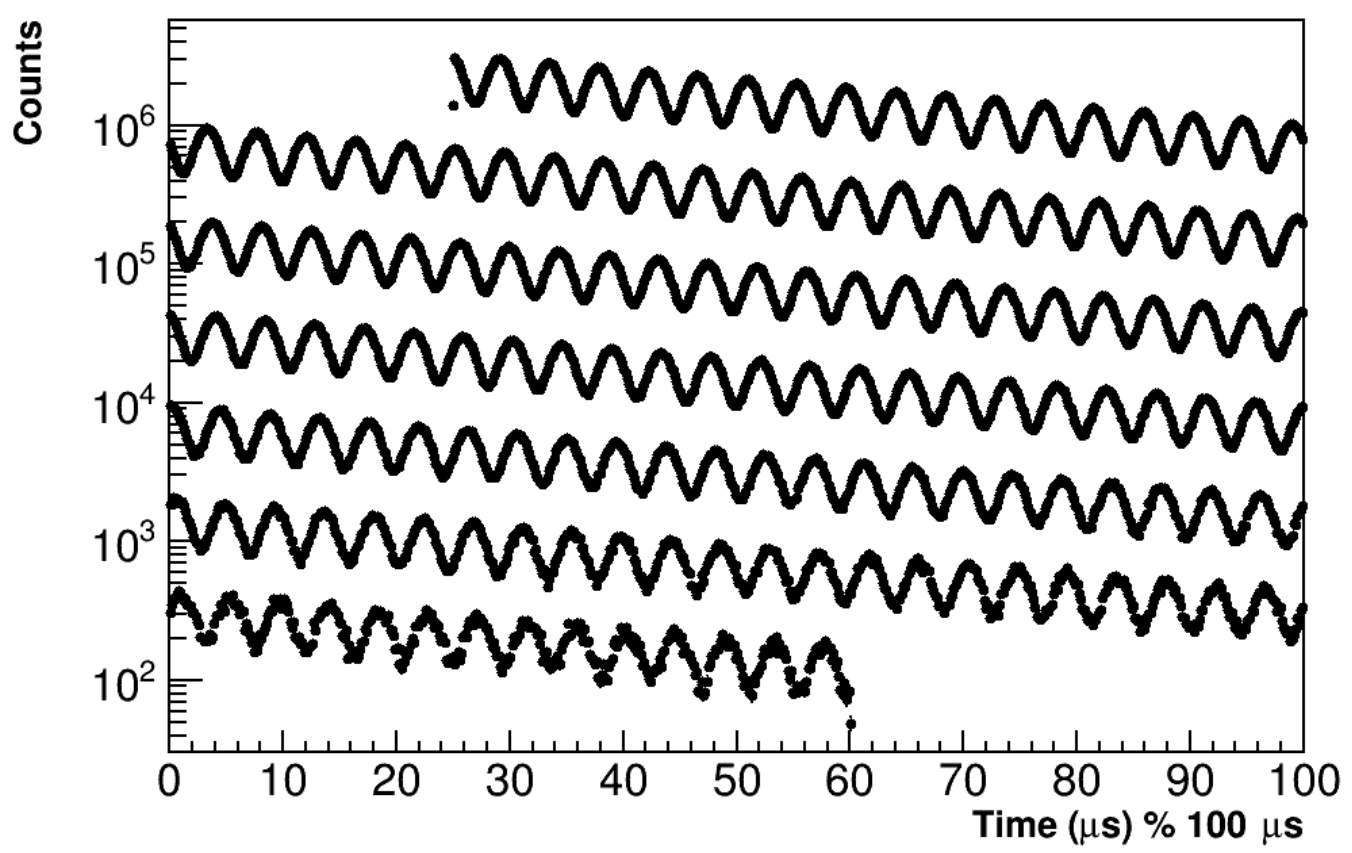

Figure 2.4: The number of detected positrons above some energy threshold $(y \sim 0.55)$ as a function of time, where the data plotted come from Run 1 and correspond to nearly $1 \times 10^{9}$ counts. The time axis is wrapped around every $100 \mu \mathrm{s}$.

simply integrating Equations 2.11 and 2.12 from $y_{t h}$ to 1,

$$
\begin{aligned}
N_{0}\left(E_{t h}\right) & \propto\left(y_{t h}-1\right)^{2}\left(-y_{t h}^{2}+y_{t h}+3\right), \\
A\left(E_{t h}\right) & =\frac{y_{t h}\left(2 y_{t h}+1\right)}{-y_{t h}^{2}+y_{t h}+3},
\end{aligned}
$$

where $y_{t h}=E_{t h} / E_{\max }$. By counting decay positrons above some energy threshold and fitting the resulting time spectrum with Equation 2.13, $\omega_{a}$ can be extracted. A sample of data adhering to such a time spectrum is shown in Figure $2 \cdot 4$.

The statistical error on the $\omega_{a}$ measurement, assuming bin errors are Gaussian and a $\chi^{2}$ minimization is used with the fit function described in Equation 2.13, is 53

$$
\frac{\sigma_{\omega_{a}}}{\omega_{a}}=\frac{\sqrt{2}}{\sqrt{N_{\text {total }}} A \gamma \tau_{\mu} \omega_{a}},
$$


where $N_{\text {total }}$ is the total number of counts included in the above-threshold time spectrum. This equation assumes a weighting of one for every count included in the fitted time spectrum. Other weighting schemes exist which slightly improve the statistical precision of the $\omega_{a}$ measurement [53], but they were not used in the analysis presented in this dissertation. What Equation 2.16 reveals is that the statistical precision of $\omega_{a}$ is maximized when the quantity $N A^{2}$ is at a maximum. It was found for E989 that the optimal energy threshold was about $1.7 \mathrm{GeV}$ as shown in Figure 5.5, which includes detector acceptance effects and corresponds to an asymmetry of about $A=0.37$. Equation 2.16 can be rearranged in order to solve for the number of positrons needed to be collected above threshold for a specific precision goal. For a statistical error of $100 \mathrm{ppb}$ on $\omega_{a}$, the required number of positrons above threshold is approximately $170 \times 10^{9}$, determined from the values $A=0.37, \gamma=29.3$ (described later), $\tau_{\mu}=2.2 \mu \mathrm{s}$, and $\omega_{a}=1.44 \mathrm{rad} / \mu \mathrm{s}$. This statistical error of $\omega_{a}$ combined with the systematic uncertainties given in Table 5.9, provide the total error on $\omega_{a}$.

\subsection{Measuring the magnetic field}

In order to measure the magnetic moment of the muon to $140 \mathrm{ppb}$, the field needs to be both highly uniform, and measured to extreme precision. The E989 goal for the field measurement is $70 \mathrm{ppb}$. As shown in Equation 2.6, the measurement of the magnetic field has equal weight to that of the precession frequency. A crosssection of the magnetic storage ring used in E989 is shown in Figure 2.5. It is an approximately $14 \mathrm{~m}$ diameter $\mathrm{C}$ magnet, where the muons are stored within a $4.5 \mathrm{~cm}$ radius cylindrical storage region at the center of a $1.451 \mathrm{~T}$ magnetic field. This corresponds to an approximately $0.28 \mathrm{~m}^{3}$ or $10 \mathrm{ft}^{3}$ total volume around the inside of the ring. The magnetic field is made uniform by manipulating many magnetic 'knobs' built into the $g-2$ storage ring, including the main magnet current, pole 


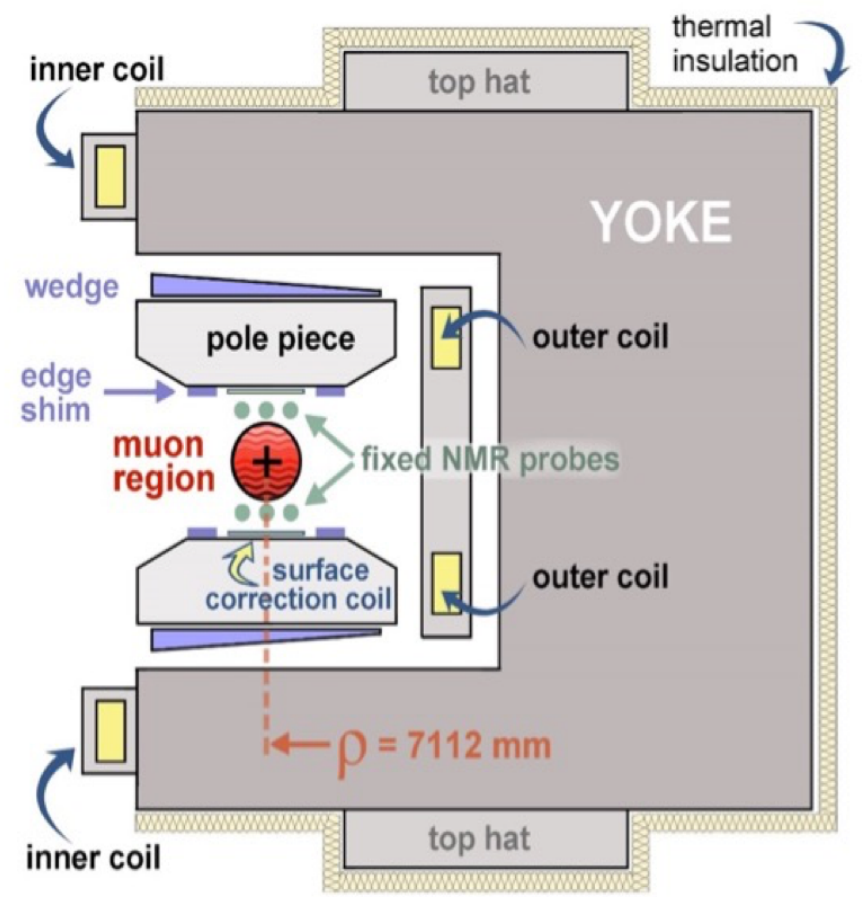

Figure 2.5: Cross-section of the $g-2$ magnet. The muons live in the $4.5 \mathrm{~cm}$ radius circular storage region shown in red. The main magnetic field is excited by superconducting coils shown in yellow. Pole pieces, wedges, edge shims, top hats, and other magnetic knobs allow for subppm level tuning of the magnetic field.

pieces, wedges, top hats, and thousands of small magnetic shims placed around the storage region. There is also an active feedback system which stabilizes the average magnetic field over time using current carrying coils near the storage region. After a several month shimming campaign by many members of the field team, a precision on the magnetic field of approximately $25 \mathrm{ppm}$ RMS (root mean square) was achieved.

The magnetic field is measured using a nuclear magnetic resonance (NMR) technique, hence the measurement on $\omega_{p}$ as shown in Equation 2.4. NMR was chosen as it provides a field measurement precision on the order of $10 \mathrm{ppb}$ with negligible statistical uncertainty [54]. NMR probes consist of pickup coils located around a sample of protons in some fluid, typically water or petroleum jelly. The pickup coils deliver a " $\pi / 2$ pulse" which rotates the proton sample magnetization $90^{\circ}$ out of phase 


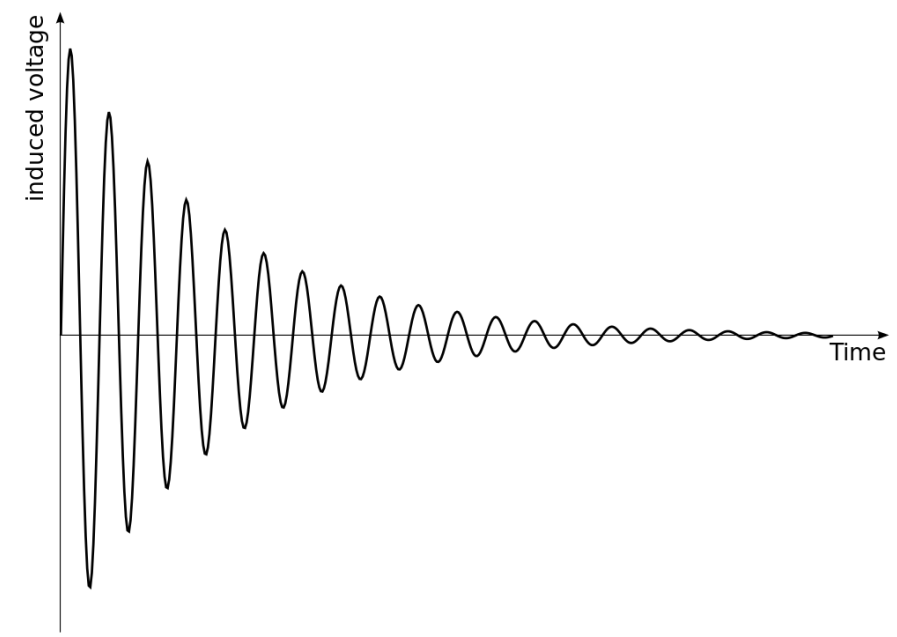

Figure 2.6: An example FID signal. The current picked up in the coils around the proton sample will oscillate as the spins precess around the main magnetic field vector, and decay as the spins return to alignment with the external field.

from equilibrium. The proton spins will begin precessing at the Larmor frequency $(\approx 61.79 \mathrm{MHz})$. As the spins interact with local magnetic field gradients and inhomogeneities, the magnetization of the proton sample will relax back to equilibrium with the external field, typically on the order of several milliseconds. This so-called free-induction decay signal (FID), an example of which is shown in Figure 2.6, is measured using the same pickup coils that delivered the initial $\pi / 2$ pulse

Technically, it is not solely $\omega_{p}$ that needs to be measured. What really matters is the average magnetic field that the muons see, namely the time-averaged spatiallyweighted magnetic field. The scheme devised to measure this is two-fold. First, the magnetic field in the muon storage region is measured by a trolley which travels around the inside of the ring. This trolley holds 17 NMR probes which measure the field at approximately 6000 locations around the inside of the ring. However, because the trolley cannot be in the storage region when the muons are present in the ring, during data taking it is retracted and the field is instead monitored by 378 fixed NMR 


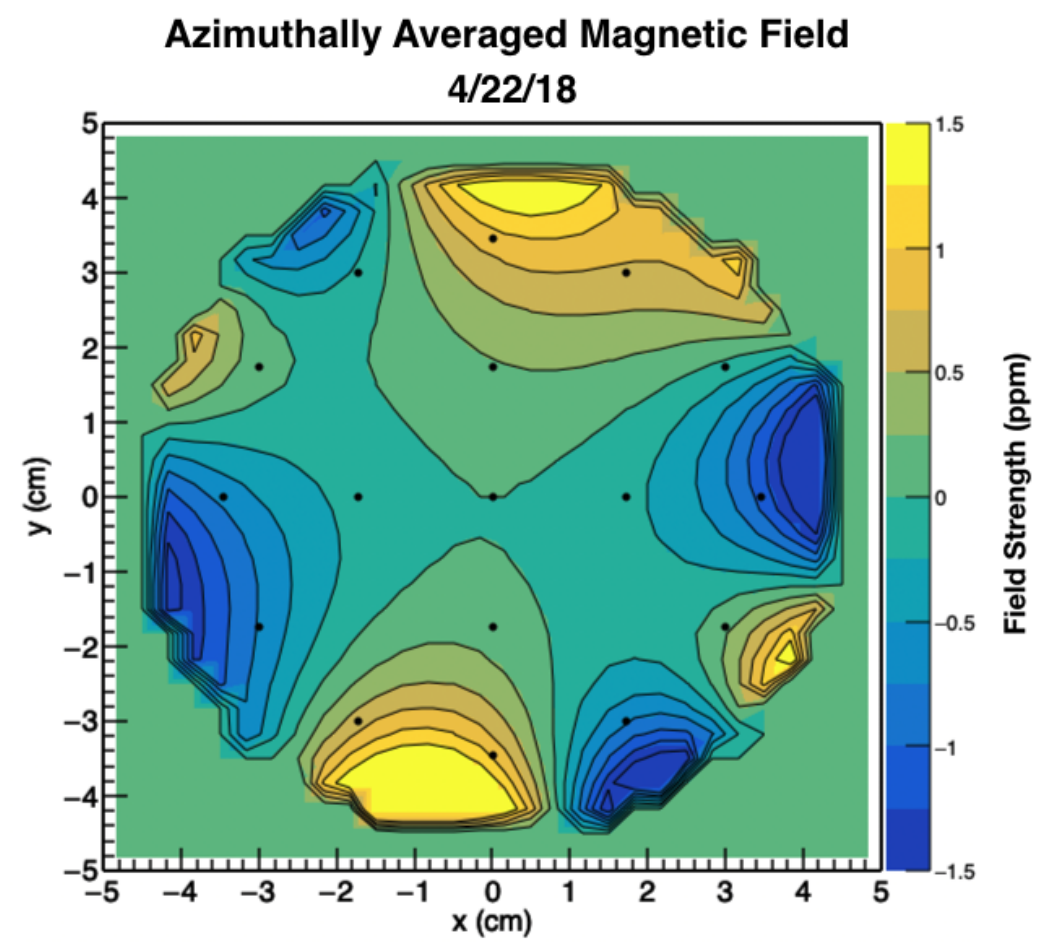

Figure 2.7: A sample of the azimuthally-averaged magnetic field within the storage region, taken at the start of the $60 \mathrm{~h}$ dataset, courtesy of R. Osofsky [55]. The main dipole field measured at the center of the storage region has been subtracted from the plot. The scale of the field differences is approximately $\pm 1.5 \mathrm{ppm}$. The black dots in the picture correspond to the location of the 17 trolley probes.

probes located in the high magnetic field region, just outside the storage region on the outside of the vacuum chambers. The prescription is that the storage ring field is measured every few days by the trolley probes, and the field between trolley runs is interpolated using measurements from the continually-sampling fixed probes. In this way the magnetic field can be mapped over time and over the space in which the muons are stored. A sample of the azimuthally-averaged magnetic field measured with trolley and fixed probes is shown in Figure $2 \cdot 7$.

Lastly, it is the free proton precession frequency in the field that is of interest, but the frequency that the trolley probes measure will be different due to the molecular properties of the proton sample as well as the material properties of the probe itself. 
The frequency of the induction signal that the probes measure can be re-cast as

$$
\omega_{p, \text { probe }}=\omega_{p, \text { free }}\left(1-\sigma\left(\mathrm{H}_{2} \mathrm{O}, \mathrm{T}\right)+\delta_{b}+\delta_{p}+\delta_{s}\right)
$$

where $\sigma\left(\mathrm{H}_{2} \mathrm{O}, \mathrm{T}\right)$ is the temperature-dependent diamagnetic shielding of protons in a water molecule, and the $\delta$ 's come from corrections due to the bulk susceptibility of the water sample, paramagnetic impurities in the water sample, and the magnetic effects of the probe itself, respectively [54]. In order to correct for these effects an additional special probe is used, the 'plunging probe', which operates in a single azimuthal section of the ring which has been shimmed to extra uniformity. This probe is made of materials which are designed to reduce the effects listed in Equation 2.17, and has been characterized in a dedicated highly uniform solenoidal magnetic field [56]. It is located inside the vacuum chamber and moves into the storage region to measure the field at each of the 17 trolley probe locations, using a three dimensional motion system. By using this probe, the calibration to the free proton precession frequency can be transmitted to each of the trolley probes, providing an absolute scale for the measurements inside the muon storage region. The uncertainty on the calibration procedure is estimated to be $35 \mathrm{ppb}$, about half the total permitted by the experimental error budget of $70 \mathrm{ppb}$.

Other pieces of the systematic uncertainty include the calibrations of the probes, errors in the trolley measurements, the interpolation with the fixed probes, the uncertainty of the magnetic field relative to the muon distribution, etc. See Table 2.1.

\subsection{Production of polarized muons}

The muons injected into the storage ring must be polarized in order to measure $\omega_{a}$. The BNL E821 experiment observed on the order of 10 billion decay positrons above threshold, and its final result was statistics limited. In order to reach the goal 


\begin{tabular}{lc}
\hline \multicolumn{2}{c}{ Magnetic Field Measurement } \\
\hline \hline Source of uncertainty & E989 Goal (ppb) \\
\hline Absolute calibration of standard probe & 35 \\
Calibration of trolley probes & 30 \\
Trolley measurements & 30 \\
Fixed probe interpolation & 30 \\
Muon distribution weighted average & 10 \\
Time dependent external fields & 5 \\
Others & 30 \\
Quadrature sum & $\sim 70$ \\
\hline
\end{tabular}

Table 2.1: Systematic errors in the magnetic field measurement. Unlisted sources of error include the measurement of higher field multipoles, trolley temperature and power supply voltage response effects, and eddy currents from the kicker, among others.

of $140 \mathrm{ppb}$, approximately 20 times that number of positrons needs to be gathered. Fermilab has the facilities to produce the required number of polarized muons.

Polarized muon beams are constructed using the physics of pion decay. Using the same parity-violation and spin momentum conservation logic as explained in the discussion of muon decay, it is determined that pion decay produces muons that are $100 \%$ polarized in the pion rest frame, due to the pion having zero spin. It is also important to note that pions decay to muons with a $\sim 99.98 \%$ branching ratio due to the parity violating nature of the weak interaction [57]. Thus by producing a large number of polarized pions a large number of polarized muons can be acquired.

The production of polarized muons for E989 at the Fermilab accelerator complex involves a number of stages. A map of the various relevant accelerator beam-line components is shown in Figure 2.8. Details of the full accelerator production of polarized muons can be found in Reference [58], and here a summary of the process will be given. First, $\mathrm{H}^{-}$ions are produced and accelerated in a linear accelerator. They are stripped down to protons and then transported to a $75 \mathrm{~m}$ radius circular 
storage ring called the "booster," which accelerates them up to $8 \mathrm{GeV} / \mathrm{c}$ and batches them together. A single booster batch contains on the order of $4 \times 10^{12}$ protons. The protons are then injected into a ring called the "recycler," which separates them into four separate bunches of $1 \times 10^{12}$ protons, each with a time width of approximately $120 \mathrm{~ns}$. (This is less than the cyclotron period of the storage ring of $149 \mathrm{~ns}$.) This re-bunching process is done in order to reduce the instantaneous rates observed by the detectors, and thus reduce the level of pileup in the E989 detectors; see Section 3.2.1. For a single accelerator supercycle of $1.4 \mathrm{~s}$, E989 receives four booster batches corresponding to sixteen recycler bunches at an average rate of $11.4 \mathrm{~Hz}$, with the time separation between bunches greater than $10 \mathrm{~ms}$. The timing structure is shown in Figure 2.9, where trains of eight bunches are sent to E989 with some time separation between them $2^{2}$, Although it remains relatively constant, this timing structure may be modified in response to the requirements of other experiments.

Each bunch is sent to a target hall, where it is directed on to an Inconel target. This Inconel target is made of a nickel-iron alloy, and is optimized for the production of large numbers of pions with a small momentum spread, approximately $1 \times 10^{-5} \pi^{+}$ per proton on target (POT) with $|d p / p|<2 \%$ [58]. The pions are focused by the magnetic field of a lithium lens, located just downstream of the production target. The lens, a cylinder of lithium $1 \mathrm{~cm}$ in radius and $15 \mathrm{~cm}$ long, carries a large current down its length which provides a radial focusing effect for particles passing down the cylinder [59]. A pulsed magnet just after the lithium lens is then used to select pions centered at $3.115 \mathrm{GeV}$. By extension, residual particles with the same momentum, mainly protons, are also selected.

The resultant pion beam and these residual particles are then injected into another

\footnotetext{
${ }^{2}$ These eight bunches are sometimes referred to as pulses, and the gathered data are tagged by which bunch or pulse they originated from.
} 


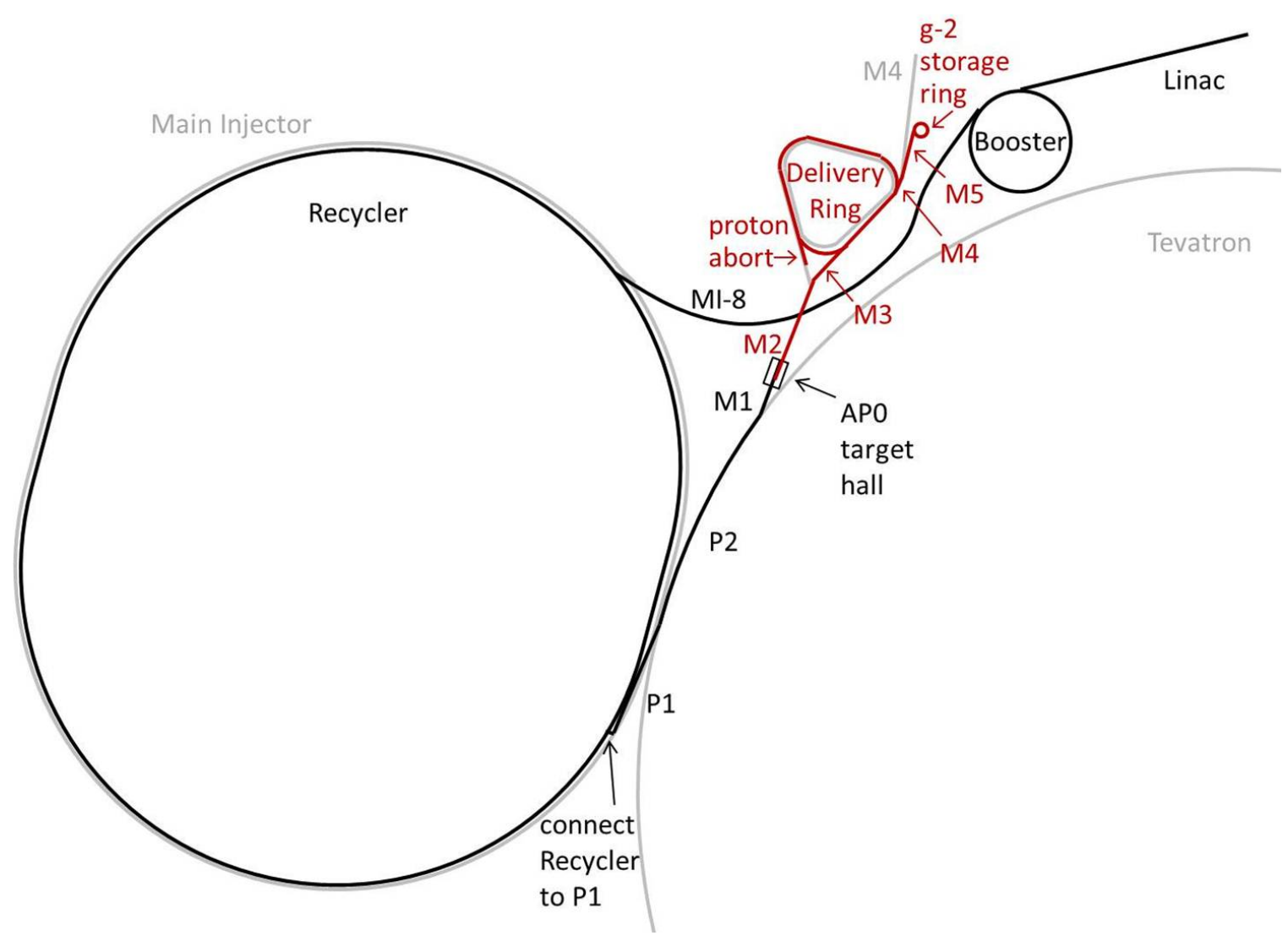

Figure 2.8: The layout of accelerator beam-line components Fermilab uses to provide polarized muons to E989. Protons start in the Linac, traverse around the Booster and then Recycler, and are converted to pions at AP0. The pions are gathered and then decay to muons in the Delivery Ring before being sent to the $g-2$ storage ring. [54].

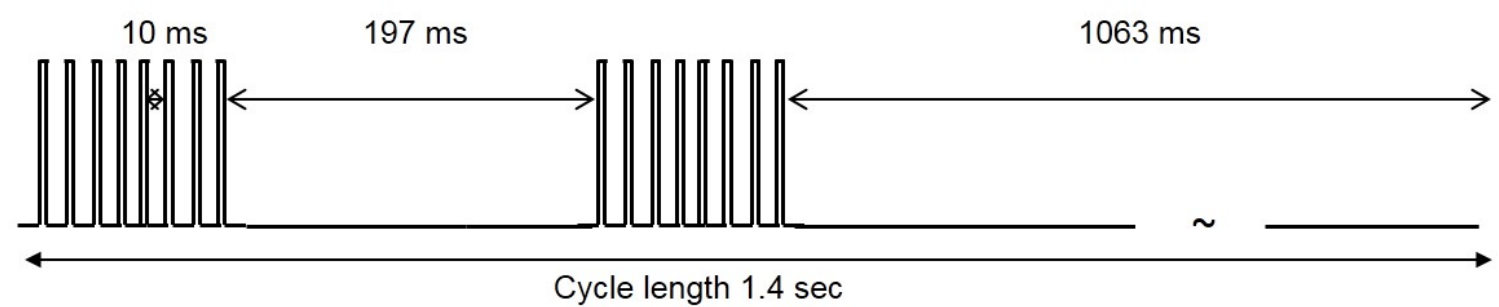

Figure 2.9: General timing structure of beam pulses sent to E989. 
ring called the "delivery ring," which holds the pions while they decay to muons ${ }^{3}$, Here it should be noted that in a pion beam the highest and lowest energy decay muons are forwards and backwards polarized, respectively. The delivery ring is used to momentum select polarized decay muons and separate out the non-desired particles which will have differing velocities compared to the muons, reducing the contamination in the final polarized muon beam [58. Forward emitted polarized muons are momentum selected at their decay energies of $3.094 \mathrm{GeV}$ with $\Delta p / p=2 \%$. Particles that fall outside the momentum acceptance are lost, and the residual protons are kicked into a beam dump. The polarized muon beam is then sent to the E989 building where it passes through four magnetic quadrupole focusing magnets before being injected into the E989 storage ring.

\subsection{Injection of muons}

The injection of the muon beam into the E989 storage ring is a specialized process. In order to produce the main magnetic field and measure it to the precision required, the storage ring must be a single monolithic magnet with no end effects. This prohibits the usual design of separated magnetic elements through which the muons might be injected. Therefore the beam must be injected through the storage ring magnet yoke. This introduces the design constraint where the main magnet field must be eliminated within the injection tunnel, such that muons are not lost due to deflection into the magnet itself. Any solution to this problem must have it's cancellation field localized to the injection tunnel, such that there is no residual fringe field that contaminates the main storage ring dipole field.

A specialized magnet called the "Superconducting Inflector" magnet, or just inflector, is used to solve these design constraints. This inflector is placed just after a

\footnotetext{
${ }^{3}$ By the time the pions have gotten to the delivery ring, most of them have already decayed to muons.
} 


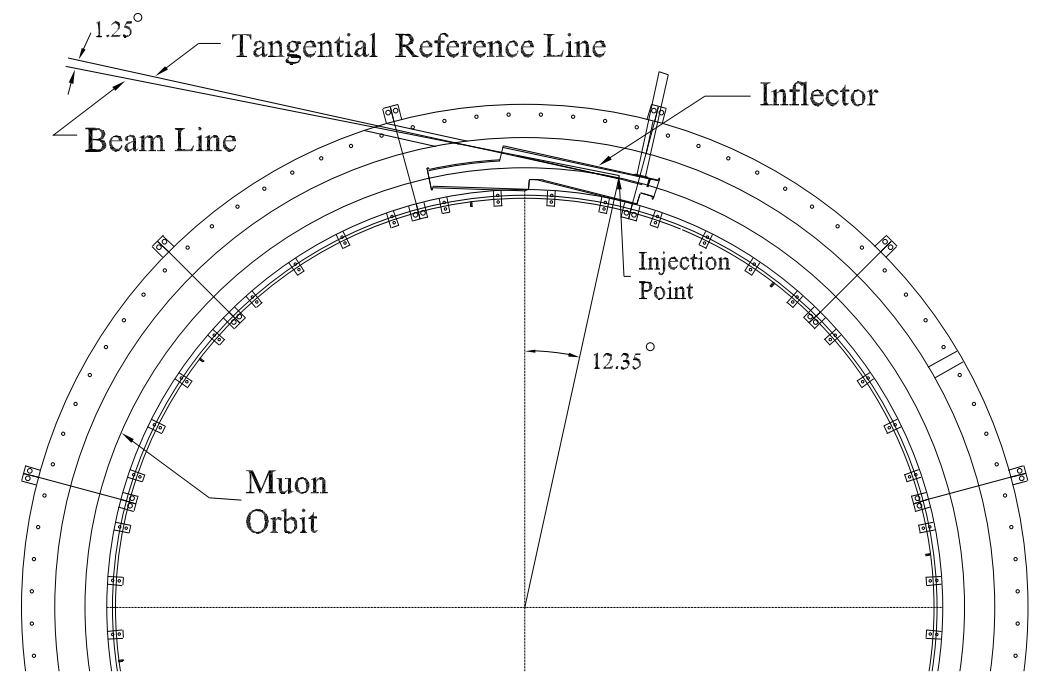

Figure 2.10: A plan view of the inflector and injection point into the storage ring 60 .

bored out tunnel in the storage ring magnet yoke, on the inside of the $\mathrm{C}$ shape. See Figure $2 \cdot 10$ for a view of the injection point. The inflector has an $18 \mathrm{~mm}$ wide by $56 \mathrm{~mm}$ high aperture through which the muons must pass down its $1.7 \mathrm{~m}$ length. The inflector is made up of superconducting coils wrapped in a truncated double cosine theta design around an aluminum mandrel $[60]$. See Figure 2·11. This design serves to contain the majority of the inflector magnet field, while eliminating the the storage ring field for the muons passing down its length. The inflector is contained within a superconducting shield which traps the fringe field of the inflector such that the storage ring magnet field is unaffected. As shown in Figure 2.11, windings cover both ends of the inflector such that an appreciable fraction of muons are lost due before being injected into the ring. In order to increase the muon flux for future runs of E989, a new inflector magnet is being designed with open ends [54]. 


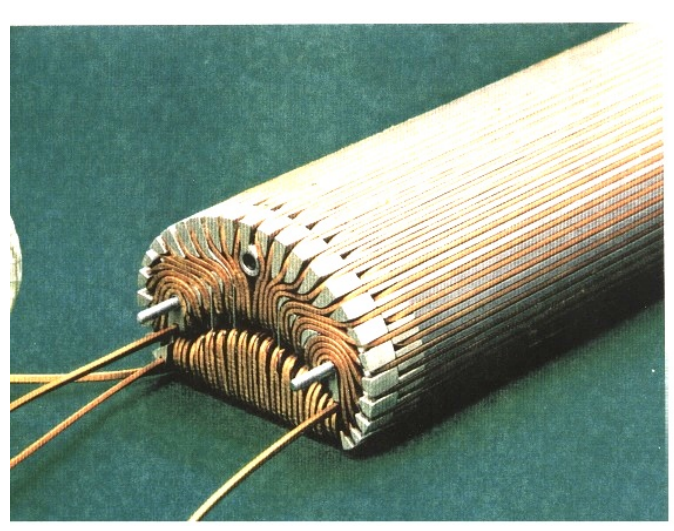

(a) End view of the inflector.

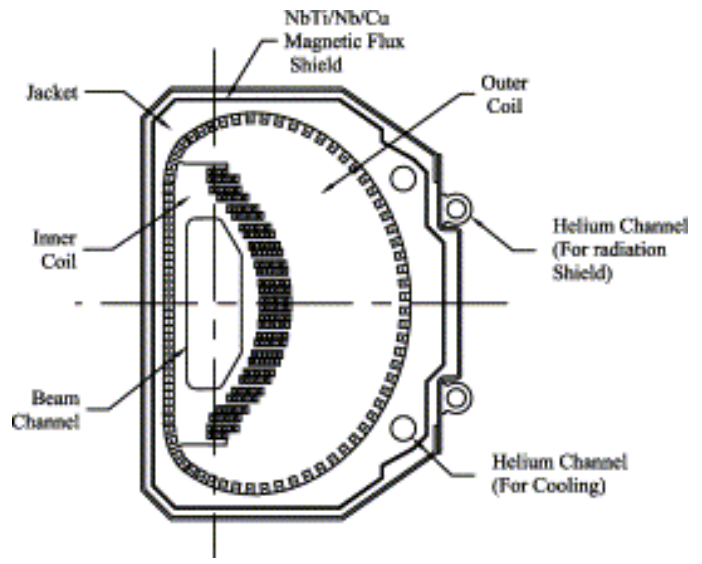

(b) Cross section view of the inflector.

Figure 2.11: The inflector magnet (left) and a cross section view of the inflector windings and associated shield (right) [60].

\subsection{Storage of muons}

Once the muons have been injected into the ring, they will begin orbiting clockwise around the ring, decaying with a lifetime of approximately $64.44 \mu \mathrm{s}$. The E989 experiment and storage ring are shown in Figure 2·12, By necessity, the inflector must be out of the stored muon beam path, otherwise a large fraction of the muons would be lost upon the return to the injection point as the muons would strike the inflector. Therefore the muon beam must be kicked to move the beam path from the injection orbit onto the central orbit of the storage ring. The muon beam must also be contained vertically. To perform the former, a magnetic "kicker" is used to shift the orbits of the muons. To perform the latter, a series of electrostatic quadrupoles focus the beam vertically. Approximately $2 \%$ of the injected muons are stored with $\Delta p / p=0.1 \%$ centered around $3.094 \mathrm{GeV} / \mathrm{c}$, corresponding to a design goal of $\mathcal{O}(10,000)$ stored muons per fill. 


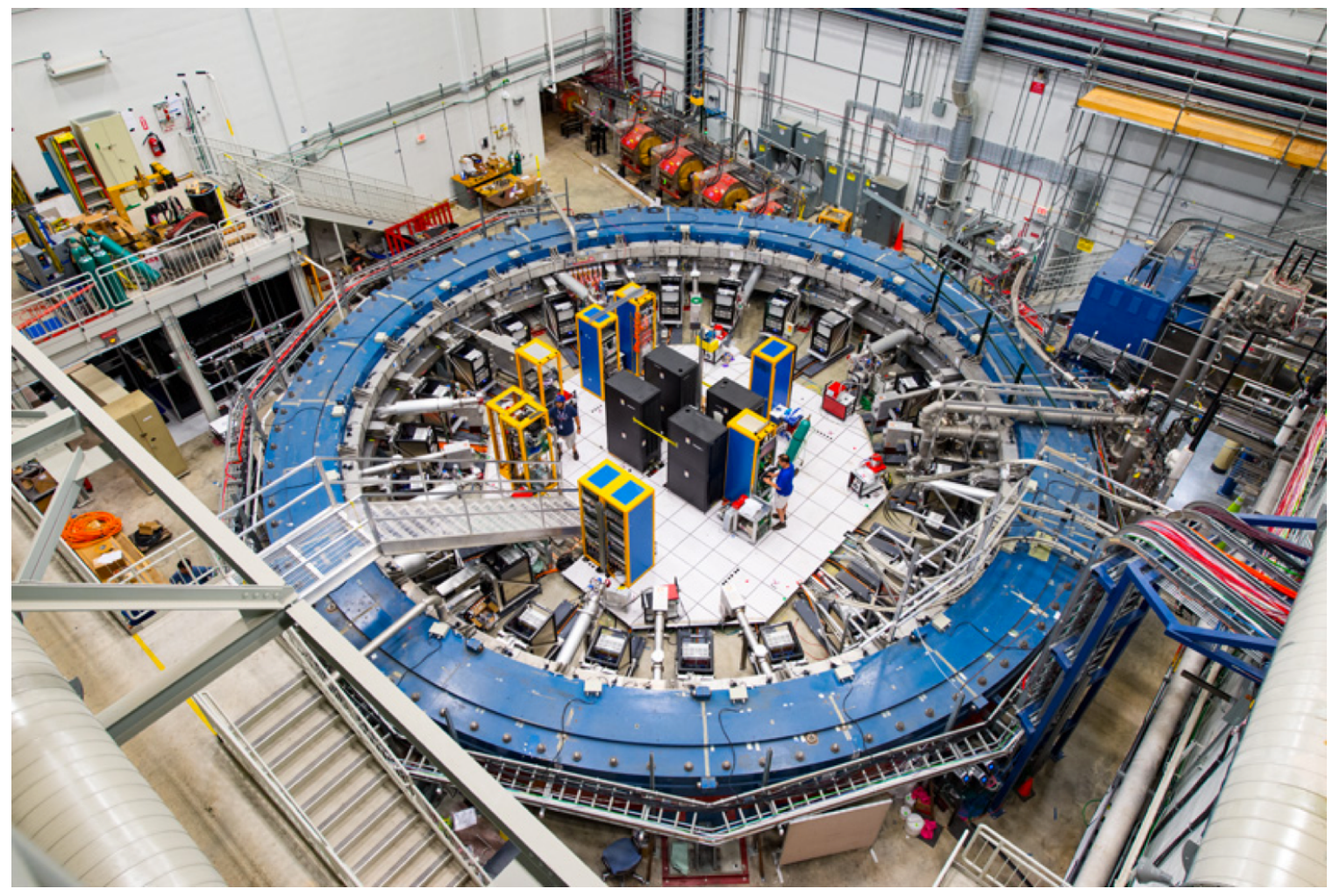

Figure 2.12: The E989 experiment. The blue storage ring can be seen to surround a variety of detectors and electronics. Muons from the accelerator enter at the top of the picture, and are transported to the storage ring through a series of magnetic quadrupoles. Muons are injected through the inflector into the ring, where they orbit in a clockwise direction. The ring is approximately $16 \mathrm{~m}$ in diameter to the outside edge of the ring, and about $3 \mathrm{~m}$ high. People in the center of the ring give a sense of scale to the picture. 


\subsubsection{Kicker}

The kicker is made up of three separate pulsed magnets located $90^{\circ}$ from the exit of the inflector, where the injection orbit crosses the central orbit. The placement of the kickers is shown in Figure $2 \cdot 13$. The kicker must be located within the precision magnetic field of the ring, and must therefore contain no magnetic elements in the hardware itself. For this reason each kicker magnet is made up of two thin $1.27 \mathrm{~m}$ long aluminum plates, separated by $10 \mathrm{~cm}$, which carry the current used to create the kicking magnetic field. Due to the bunched nature of the muon beam and the short cyclotron period of $149 \mathrm{~ns}$, ideally the kicker moves all injected muons onto the central orbit and then turns off quickly such that by the time the muons orbit back around to the kicker there is no residual kick to the beam. Any residual eddy currents must die away quickly enough such that the magnetic field seen by the stored muons is unperturbed. The design deflection of the beam is approximately 10 mrad using a vertical pulsed field of around 300 Gauss (corresponding to kicker plate voltages of $\mathcal{O}(155 \mathrm{kV}))$ with a pulse length of about $120 \mathrm{~ns}[54]$. The operational kicker performance in Run 1 was less, and is described in Section 2.8.

\subsubsection{Electrostatic quadrupoles}

There are four electrostatic quadrupoles located around the ring as shown in Figure $2 \cdot 13$, which provide vertical focusing for the beam. Although the quadrupoles are defocussing in the horizontal plane, the main dipole magnetic field provides net horizontal focusing. Just as with the kickers, the quadrupoles must be operated in vacuum. E989 uses electrostatic focusing elements instead of magnetic ones in order to avoid magnetic field gradients which would limit the precision of the magnetic field measurement. The quadrupoles occupy $43 \%$ of the ring circumference, with four quads having been chosen in order to maximize the symmetry of the beam motion, 


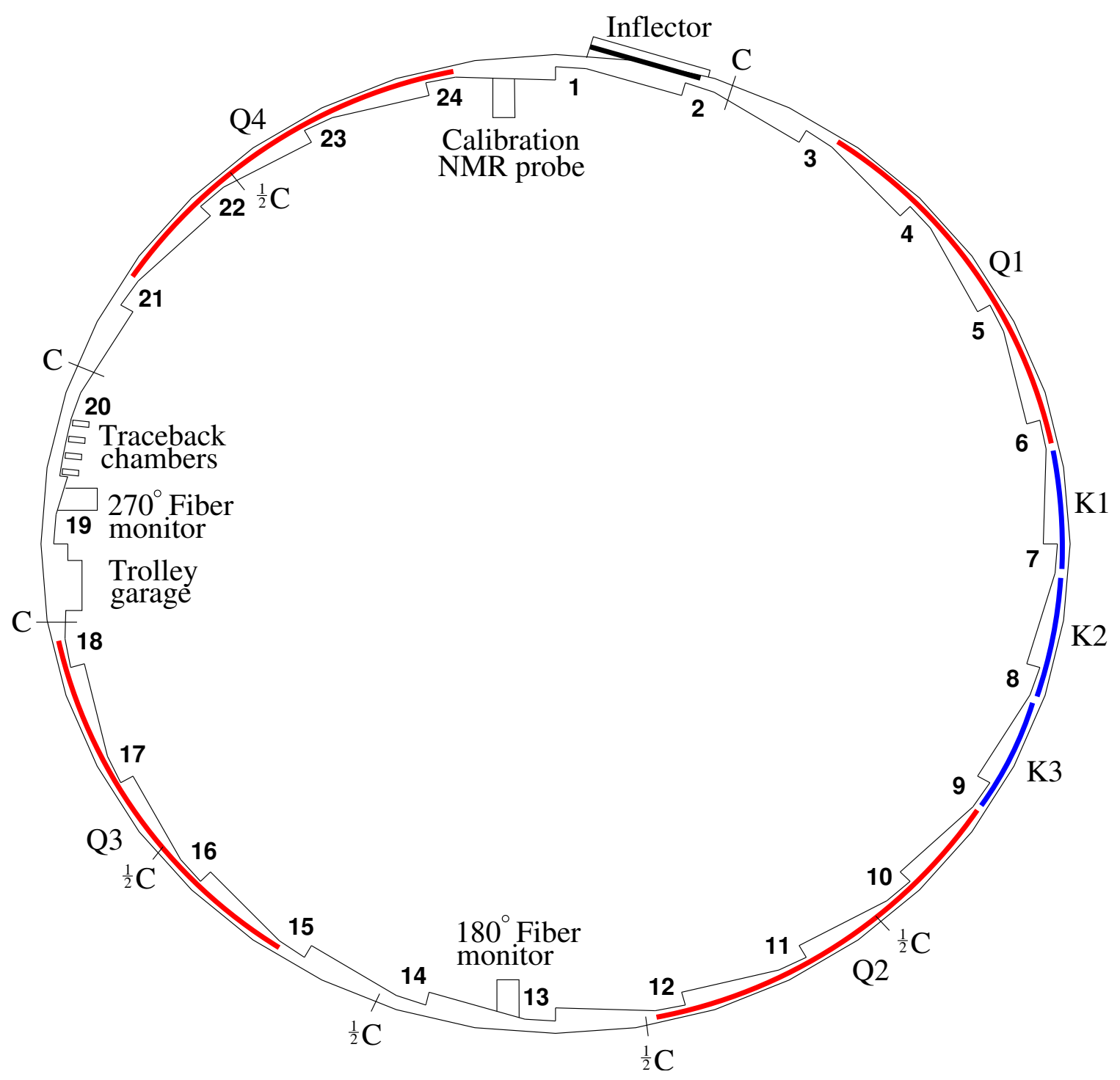

Figure 2.13: A map of the vacuum chambers in E989. K1-K3 show the locations of the kicker magnets, while Q1-Q4 show the locations of the electrostatic quadrupoles. Also shown is the location of the inflector, the two fiber monitors, and one of the tracker stations. 


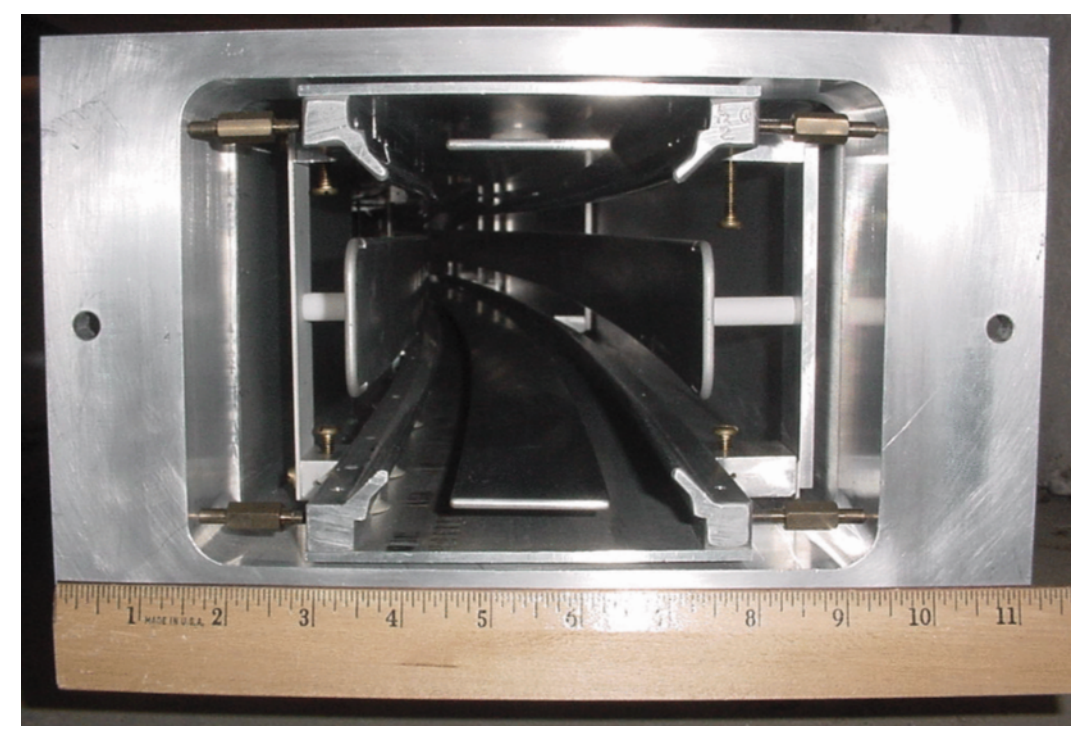

Figure 2·14: Electrostatic quadrupoles installed into a vacuum chamber [61]. There are four plates mounted to the chamber through insulator standoffs, with the distance between opposing quadrupole plates equal to $10 \mathrm{~cm}$. Also shown are the rails on which the magnetic field trolley rides. Note that as the trolley makes its way through the storage volume, it must pass between both the various sets of quadrupole and kicker electrodes.

reduce its amplitude, and leave space for other experimental elements around the ring [54]. Each quadrupole is made up of two segments, a short segment of $13^{\circ}$ and a long segment of $26^{\circ}$ corresponding to $1.61 \mathrm{~m}$ and $2.62 \mathrm{~m}$ respectively, with each segment consisting of four plates. To minimize multiple scattering of incident decay positrons, the quadrupoles are made as thin as possible. A picture of the quadrupoles installed into one of the vacuum chambers is shown in Figure 2·14. A calculation of the equipotential lines of the quadrupoles is shown in Figure $2 \cdot 15$. The original design of the quadrupoles is detailed in Reference 61].

Some of the stored muons will be lost during data taking that can adversely affect the measurement of the spin difference frequency $\omega_{a}$. See Section 5.3.3. In order to reduce the number of lost muons, a procedure called "scraping" is used to remove those muons sitting at the edge of the storage region that are more likely to be lost 


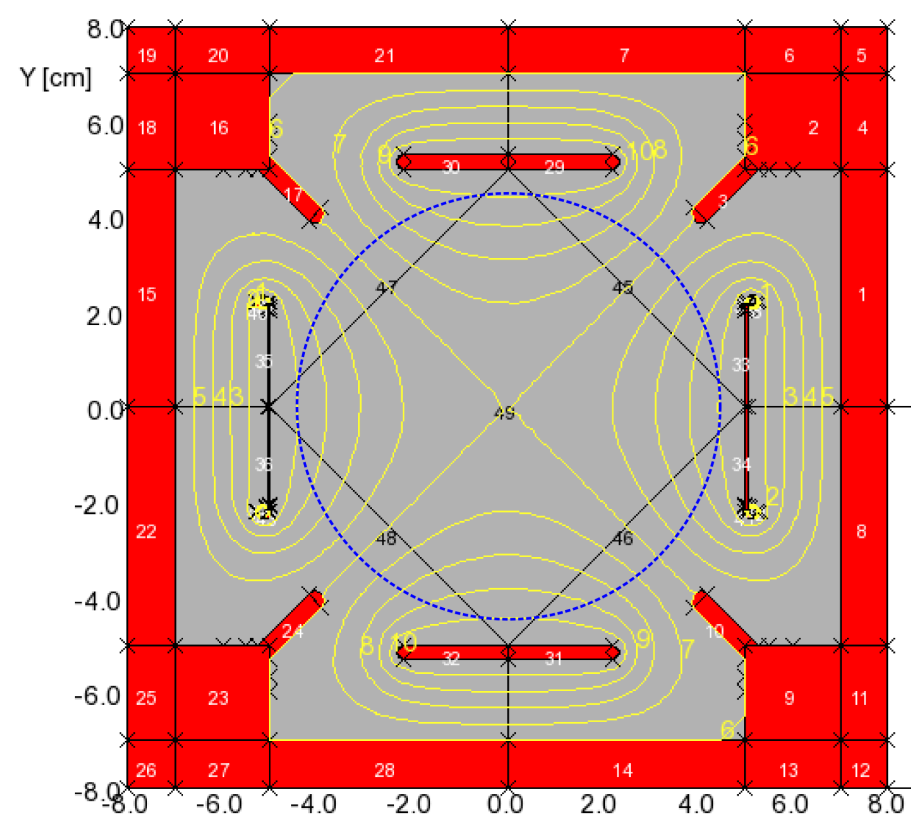

Figure 2.15: An OPERA model of the quadrupoles and their equipotential contours [54]. The top and bottom plates sit at positive voltage while the left and right plates are at negative voltage. The muon storage region is shown by the blue circle.

at later times. This scraping procedure involves powering the quadrupole voltages in an asymmetric way such that the beam is pushed to the outside of the storage region, where the edges of the beam will intersect copper collimators. These collimators have a radius of $45 \mathrm{~mm}$ and define the storage region aperture. Muons which hit the collimators will lose energy and be lost as they spiral out of the ring. The scraping procedure is performed early in the fill and ends at $8 \mu \mathrm{s}$, such that by $30 \mu \mathrm{s}$ the beam is stable and centered due to the characteristic RC time constant of the system. The operational performance of the quadrupoles in Run 1 is described in Section 2.8, where it was found that some of the quadrupoles had longer RC time constants than the design. 


\subsection{Muon beam dynamics}

Muons injected into the storage ring will occupy a region in phase space of momenta and positions defined by the injection and collimator apertures. Individual muons will undergo betatron motion within the storage ring in both the vertical and horizontal directions. The horizontal $(x)$ and vertical $(y)$ equations of motion, including the effects of the discrete quadrupoles, are given by

$$
\begin{aligned}
& x=x_{e}+A_{x}(s) \cos \left(\nu_{x} \frac{s}{R_{0}}+\phi_{x}\right), \\
& y=A_{y}(s) \cos \left(\nu_{y} \frac{s}{R_{0}}+\phi_{y}\right),
\end{aligned}
$$

where $x_{e}$ is the radial equilibrium orbit of the beam relative to $R_{0}, A_{x}(s)$ and $A_{y}(s)$ are the amplitudes of the motions containing the effects of the discreteness of the quadrupoles, and $s$ is the arc length of the trajectory. Here $\nu_{x}$ and $\nu_{y}$ are the socalled horizontal and vertical "tunes" of the beam motion, which are ratios of the betatron frequencies to the cyclotron frequency $f_{c}$ :

$$
\begin{aligned}
& \nu_{x}=f_{x_{B O}} / f_{c}=\sqrt{1-n} \\
& \nu_{y}=f_{y_{B O}} / f_{c}=\sqrt{n}
\end{aligned}
$$

These are related to the field index $n$, where the field index characterizes the strength of the electrostatic focusing in relation to the magnetic field strength:

$$
n=\frac{\kappa R_{0}}{\beta B_{0}}
$$

where $\kappa$ is the electric quadrupole gradient, $B_{0}$ is the magnetic field strength, $R_{0}$ is the central storage ring radius, and $\beta \cdot c$ is the velocity of the muon beam. Technically $n$ is the the average field index around the ring, where this approximation is justified due to the four-fold symmetry of the discrete quadrupoles and the fact that the betatron 


\begin{tabular}{lcccc}
\hline \multicolumn{5}{c}{ Muon Beam Frequencies } \\
\hline \hline Name & Symbol & Expression & Frequency $(\mathrm{MHz})$ & Period \\
\hline$g-2$ & $f_{a}$ & $a_{\mu} B e / 2 \pi m c$ & 0.23 & $4.365 \mu \mathrm{s}$ \\
cyclotron & $f_{c}$ & $v / \pi R_{0}$ & 6.71 & $149.2 \mathrm{~ns}$ \\
horizontal betatron & $f_{x_{B O}}$ & $\sqrt{1-n} f_{c}$ & 6.34 & $158 \mathrm{~ns}$ \\
vertical betatron & $f_{y_{B O}}$ & $\sqrt{n} f_{c}$ & 2.21 & $452 \mathrm{~ns}$ \\
coherent betatron & $f_{C B O}$ & $f_{c}-f_{x_{B O}}$ & 0.37 & $2.703 \mu \mathrm{s}$ \\
vertical waist & $f_{V W}$ & $f_{c}-2 f_{y_{B O}}$ & 2.31 & $433 \mathrm{~ns}$ \\
\hline
\end{tabular}

Table 2.2: Frequencies seen in the $g-2$ experiment due to beam motion. Parameter values are from a subset of Run 1 corresponding to an $n$ value of 0.108 or a quadrupole voltage of $18.3 \mathrm{kV}$.

oscillations have wavelengths much greater than the length of the quads. A table of the important frequencies in E989 is shown in Table 2.2. Lastly, the maximum angular acceptance of the ring can be determined from the betatron oscillations and the field index as

$$
\begin{aligned}
& \psi_{x_{\max }}=\frac{x_{\max } \sqrt{1-n}}{R_{0}}, \\
& \psi_{y_{\max }}=\frac{y_{\max } \sqrt{n}}{R_{0}},
\end{aligned}
$$

where $x_{\max }$ and $y_{\max }$ are both equal to the radius of the storage region aperture at $45 \mathrm{~mm}$.

As the muon beam goes around the ring, the muons will experience local field gradients and inhomogeneities. The muons will inevitably pass through the present perturbations many times. As long as the muons do not pass through the perturbations at the same phases of their respective betatron motions, the amplitudes of any would-be resonant oscillations will not grow. The tunes are chosen to avoid these resonances by having the muons sample the entire azimuth of the ring equally, thus keeping the beam stored. The general resonance condition is 62

$$
a \nu_{x}+b \nu_{y}=c,
$$




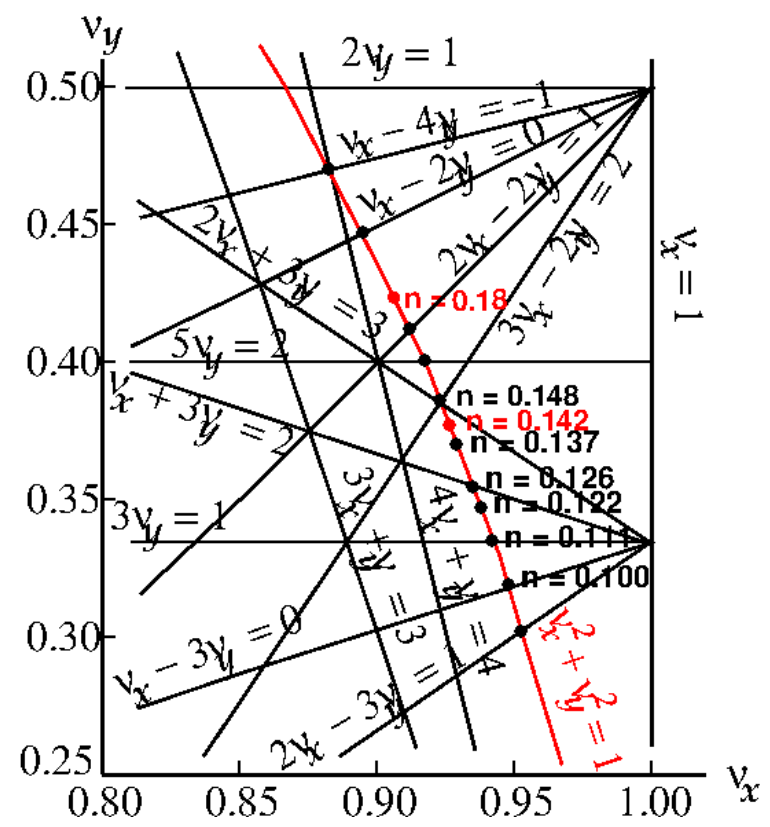

Figure 2.16: The tune plane, with the $\nu_{x}^{2}+\nu_{y}^{2}=1$ constraint in red. The value of $n$ lies on this circle. Intersections with the black lines correspond to potential resonances. The original design points for the E989 $n$ values are shown by the red points, but due to hardware issues smaller $n$ values of 0.108 and 0.120 were used.

where $a, b$, and $c$ are integers. We know from Equation 2.20 that

$$
\nu_{x}^{2}+\nu_{y}^{2}=1
$$

which constrains the available $n$ values that can be chosen. Figure $2 \cdot 16$ shows the intersections of the resonant lines of Equation 2.23 along with the circular arc of Equation 2.24 in the tune plane, for which a chosen value of $n$ will lie on a resonance. The operational $n$ values and corresponding quadrupole voltages in Run 1 are described in Section 2.8

\subsubsection{Coherent betatron oscillation}

The muon beam consists of many muons, each individually undergoing betatron oscillations. If the phase of the oscillations of the individual muons are incoherent, 
then the beam can be thought of as a static entity, constant in time around the ring. However, due to injection and kicker effects which induce a particular phase space distribution on the injected beam, the beam itself can be said to oscillate. The beam is then described by a width and mean dependent on the injection process, and the strength and phase of the kicker pulse, such that the phase distribution of the beam oscillates coherently every betatron wavelength 4 . Due to the mismatching of betatron wavelengths to the ring circumference in order to avoid resonances, a singular time slice of the distribution can be said to move around the ring over time. Individual detectors around the ring measure the beam in discrete pieces based on their individual azimuthal acceptances, where these acceptances depend on the radial and vertical characteristics of the beam. Because the radial betatron frequency is larger than half the cyclotron frequency, there is an aliasing effect such that the radial betatron motion of the beam is instead observed as an apparent slow-moving oscillation. We call the measurable signal of this coherent radial motion coherent betatron oscillation (CBO). See Figure 2.17 for a pictorial view of this phenomenon. Since the acceptance of the calorimeters depend on the beam properties, the CBO will modulate the $\omega_{a}$ signal.

The frequency of the CBO is just the beat frequency between the cyclotron frequency and the horizontal betatron frequency

$$
f_{C B O}=f_{c}-f_{x_{B O}}
$$

There is also a vertical CBO effect, but the non-aliased rate of oscillation is fast enough such that the effect tends to average out. However, the detectors are sensitive to oscillations of the vertical width of the beam, which is aliased in a similar way to the radial oscillation. Though the principles are the same, we call this effect the

\footnotetext{
${ }^{4}$ The four-fold quadrupole symmetry was chosen in order to minimize this beam 'breathing.'
} 


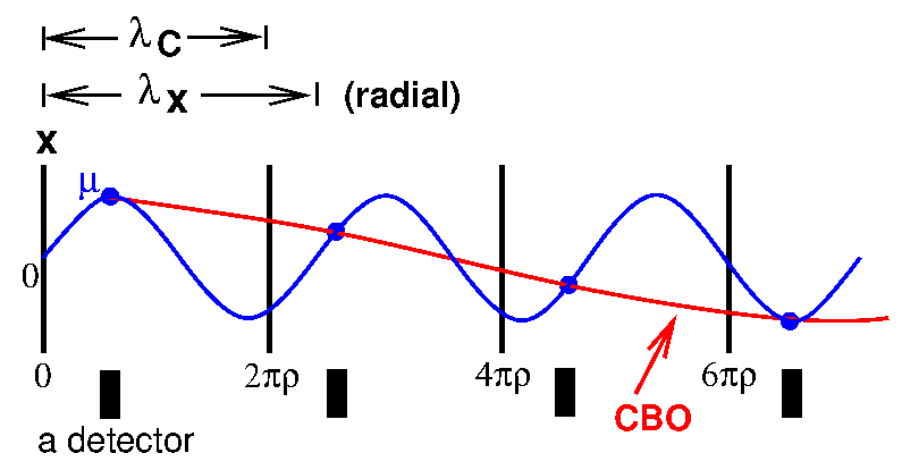

Figure 2·17: Diagram describing the coherent betatron motion of the beam. Marked by the black vertical lines are integer steps in the circumference of the ring, corresponding to the cyclotron wavelength $\lambda_{c}$. The blue line shows the motion of the beam due to the betatron oscillations with wavelength $\lambda_{x}$. Since $\lambda_{x}>\lambda_{c} / 2$, there is an aliasing effect in the observed signal, which is identified by the red line. To a single detector the beam appears to move slowly back and forth with $f_{C B O}$.

vertical waist (VW),

$$
f_{V W}=f_{c}-2 f_{y_{B O}}
$$

where the term waist refers to the minimum vertical width. Both of these frequencies are included in Table 2.2. The phase of the CBO signal varies systematically by detector, from 0-2 $\pi$ around the ring. When adding all of the detector signals together, the CBO oscillations tend to cancel out. However, due to acceptance differences between the different detectors, the $\mathrm{CBO}$ oscillations are still observable in the data. When fitting the data to extract $\omega_{a}$, these effects must be accounted for in the fit function, as will be discussed in Section 5.3.1.

\subsubsection{Beam debunching}

The muon beam is injected into the ring with a time spread of $120 \mathrm{~ns}$ and a range of momenta. At early times the beam will occupy a portion of the ring less than the whole since the cyclotron period is $\sim 149$ ns. At initial injection, detectors located at 
discrete points around the ring see a high intensity as the beam passes by and low intensity when it is on the other side of the ring. The cyclotron period of each muon is determined by its particular momentum which gives its radius of orbit. Higher momentum muons have a larger radius and therefore larger distance to travel, thus taking longer to circulate the ring than lower momentum muons. This leads to the low momentum muons in the head of the bunch catching up with the high momentum muons in the tail, such that the gaps in between each passing of the beam bunch are reduced. By $30 \mu \mathrm{s}$ the muon beam has gone around the ring two hundred times and the gaps with no beam have reduced so much that the intensity is near-uniform. The varying intensity from the beam bunching is referred to as the "fast rotation" signal. This debunching signal is seen in the data as shown in Figure 2.18. When dealing with the data and attempting to extract $\omega_{a}$, the typical procedure is to both bin out the fast rotation in periods of the cyclotron frequency, and to randomize each hit time by $\pm T_{c} / 2$ where $T_{c}$ is the cyclotron period. In this way the fast rotation is removed entirely and the effect can be ignored in the fitting procedure for $\omega_{a}$.

\subsection{Corrections to $\omega_{a}$}

Equation 2.3 is an idealized version of the spin difference frequency which ignores two important beam dynamics effects: torques exerted by the electric field and changes in the rest frame magnetic field resulting from the vertical pitching motion of the muons. Including practical experimental concerns, these two corrections must be applied to $\omega_{a}$. 

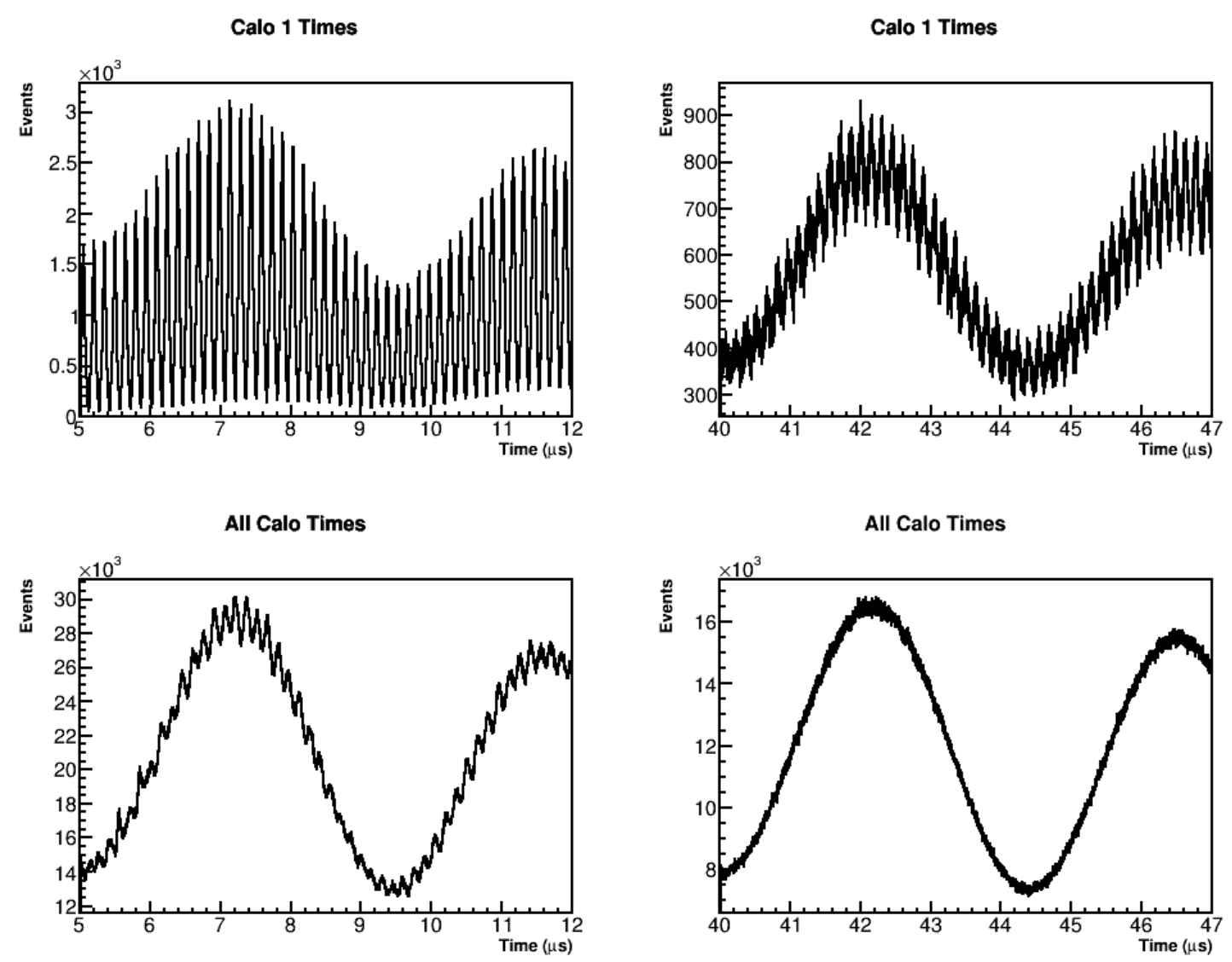

Figure 2.18: In the data, the fast rotation signal is a fast (149.2 ns) modulation on top of the slower $(4.365 \mu \mathrm{s}) \omega_{a}$ frequency. The plots here are from a subset of data from Run 1. In individual calorimeters at early times the fast rotation signal is seen to be very large, as shown on the top left. As time passes and the beam debunches, the amplitude of the fast rotation signal diminishes as shown on the top right. When adding all calorimeters together, the signal is reduced as shown in the bottom two plots, due to the fast rotation signal being unaligned in phase for the different detectors. 


\subsubsection{Electric field correction}

In the presence of an electric field, the spin difference frequency is altered to

$$
\vec{\omega}_{a}=-\frac{q}{m}\left[a \vec{B}-\left(a-\frac{1}{\gamma^{2}-1}\right)(\vec{\beta} \times \vec{E})\right]
$$

where now there is an extra term dependent on the electric field strength and the momentum of the particles. This extra term originates from the motional magnetic field $\vec{\beta} \times \vec{E}$ that relativistic particles experience in an electric field. This is necessary to include since we use electrostatic quadrupoles for vertical focusing as described above. The second term cancels to first order for a specific momentum. This "magic momentum" can be understood as the momentum at which a relativistic particle moving through an electric field has its spin exactly follow its momentum. This magic momentum is $3.094 \mathrm{GeV}$ for muon, with $\gamma \approx 29.3$. This value sets the energy and time scales of the experiment and has driven many of the design constraints, including the size of the storage ring, choice of the magnetic field magnitude, etc.

Not all muons will have the magic momentum however as described in the Section 2.6.2, and therefore a correction to the measured $\omega_{a}$ frequency needs to be applied. Approximating the storage ring as having an electric field applied over the whole azimuth of the ring, the spin difference frequency for muons with momentum $p \neq p_{m}$, where $p_{m}$ is the magic momentum, becomes

$$
\omega_{a}^{\prime}=\omega_{a}\left[1-\beta \frac{E_{r}}{c B_{y}}\left(1-\frac{1}{a \beta^{2} \gamma^{2}}\right)\right]
$$

Here the motion of the beam is assumed purely azimuthal. This additional term is the electric field correction that then serves to lower the measured $\omega_{a}$ frequency. Using the relation $p=\beta \gamma m=\left(p_{m}+\Delta p\right)$, after a little bit of simplification the electric field 
correction can be written as

$$
C_{\mathrm{E}}=\frac{\Delta \omega_{a}}{\omega_{a}}=-2 \frac{\beta E_{r}}{c B_{y}} \frac{\Delta p}{p_{m}} .
$$

The last fraction can be related to the field index described in Equation 2.21 by

$$
\frac{\Delta p}{p_{m}}=(1-n) \frac{\Delta R}{R_{0}}=(1-n) \frac{x_{e}}{R_{0}},
$$

since we know that the magic momentum muons orbit at the central radius $R_{0}$ of the storage ring. In this equation $x_{e}=\Delta R$ is the equilibrium radius of the muon beam relative to the central storage radius. Noting that the radial electric field strength for a quadrupole is

$$
E=\kappa x=\frac{n \beta c B_{y}}{R_{0}} x
$$

and assuming that it is perfectly radial, the electric field correction reduces to

$$
C_{\mathrm{E}}=-2 n(1-n) \beta^{2} \frac{x x_{e}}{R_{0}^{2}}
$$

Taking the time average of the beam motion, where $x$ is simply equal to $x_{e}$, the correction becomes

$$
C_{\mathrm{E}}=-2 n(1-n) \beta^{2} \frac{\left\langle x_{e}^{2}\right\rangle}{R_{0}^{2}} .
$$

Since the equilibrium radius of the beam is set by the momentum distribution of the muons, this electric field correction can be determined by a measurement of the momentum spread of the beam which comes from an analysis of the fast rotation 63 , 64. For the precision goal of E989, the assumptions made in this derivation are acceptable [54 and results will be cross-checked with spin-tracking simulations as was done in E821 [36]. In E821 the electric field correction was approximately 500 ppb on 
$\omega_{a}[36]$. In E989 the scale of the correction is the same considering the experimental principles are identical, Section 5.5.8.

\subsubsection{Pitch correction}

Particles injected into the $g-2$ storage ring will have a vertical component of momentum which is parallel to the magnetic field vector (hence the need for vertically focusing electrostatic quadrupoles). This will slightly reduce the magnetic field seen by the muons in their rest frames. Including this motion into the spin difference frequency, $\omega_{a}$ becomes

$$
\vec{\omega}_{a}=-\frac{q}{m}\left[a \vec{B}-a\left(\frac{\gamma}{\gamma+1}\right)(\vec{\beta} \cdot \vec{B}) \vec{\beta}\right]
$$

where now there is an extra term dependent on the vertical betatron motion of the beam. Similar to the electric field case, this term can be neglected to first order as the muon momentum is nearly all perpendicular to the field, but a correction again needs to be applied to $\omega_{a}$ to account for this effect.

Since the muons in the storage ring will be oscillating vertically as they are focused by the quadrupoles, their momentum vectors will be pitching up and down relative to the azimuthal motion. This pitch angle will oscillate as

$$
\psi=\psi_{0} \cos \left(\omega_{y} t\right)
$$

where $\psi_{0}$ is the amplitude of the oscillation and $\omega_{y}$ is the vertical betatron frequency. Shown in Figure $2 \cdot 19$ is an exaggerated example of the beam motion relative to the

vertical and azimuthal axes. Assuming that the field is purely vertical, $\vec{B}=B_{y} \hat{y}$ and that the beam motion is in the vertical-azimuthal plane,

$$
\vec{\beta}=\beta_{y} \hat{y}+\beta_{z} \hat{z}=\beta \sin (\psi) \hat{y}+\beta \cos (\psi) \hat{z}
$$




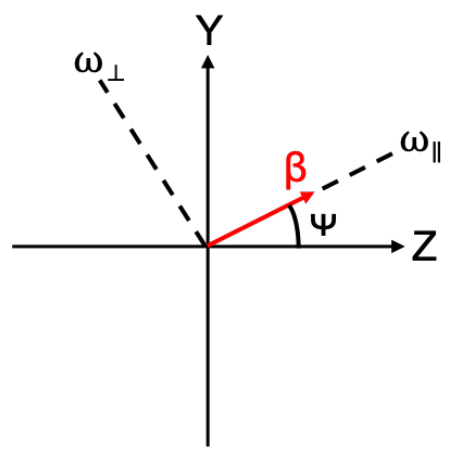

Figure 2.19: Beam motion $\beta$ relative to the vertical and azimuthal axes $\mathrm{Y}$ and $\mathrm{Z}$ respectively. $\psi$ is the pitch angle of the beam, and the dashed lines represent the parallel and perpendicular motions of the beam.

then $\omega_{a}$ becomes

$$
\vec{\omega}_{a}=-\frac{q}{m}\left[a B_{y} \hat{y}-a\left(\frac{\gamma}{\gamma+1}\right) \beta_{y} B_{y}(\beta \sin (\psi) \hat{y}+\beta \cos (\psi) \hat{z})\right]
$$

Using the small angle approximation such that $\cos (\psi) \approx 1$ and $\sin (\psi) \approx \psi, \vec{\omega}_{a}$ can be separated into its vertical and azimuthal components

$$
\begin{aligned}
\omega_{a y} & =\omega_{a}\left[1-\left(\frac{\gamma-1}{\gamma}\right) \psi^{2}\right], \\
\omega_{a z} & =-\omega_{a}\left(\frac{\gamma-1}{\gamma}\right) \psi .
\end{aligned}
$$

Looking at Figure 2.19 again, it can be seen that the spin difference frequency can be resolved into its parallel and perpendicular components $\omega_{\|}$and $\omega_{\perp}$ respectively. As the pitch angle of the beam motion oscillates about the azimuthal axis at a frequency much greater than the $g-2$ frequency, it can be assumed that the parallel component averages to 0 over time. The perpendicular oscillation of the beam then becomes the important quantity, which can be determined with a simple rotation matrix such that

$$
\omega_{a} \approx \omega_{\perp}=\omega_{a y} \cos (\psi)-\omega_{a z} \sin (\psi) \approx \omega_{a}\left[1-\frac{\psi^{2}}{2}\right]
$$

where in the last approximation the small angle approximation was used once again, 
but this time with $\cos (\psi) \approx 1-\psi^{2} / 2$. The pitch correction then is the additional term which serves to lower the measured spin difference frequency. Taking the time average,

$$
C_{\mathrm{P}}=\frac{\Delta \omega_{a}}{\omega_{a}}=-\frac{\left\langle\psi^{2}\right\rangle}{2} .
$$

The pitch angle of the beam cannot be measured directly, however the angle of the beam can be related to the vertical distribution of the beam with Equation 2.22, such that

$$
C_{\mathrm{P}}=-\frac{n}{2} \frac{\left\langle y^{2}\right\rangle}{R_{0}^{2}},
$$

where once again $n$ is the field index, $R_{0}$ is the radius of the ring at the center of the storage region, and $\left\langle y^{2}\right\rangle$ is the vertical width of the beam. The first two are known and the last can be measured experimentally by the straw tracking detectors. Just as in the case of the electric field correction, the assumptions made in this derivation are acceptable for the precision goal of E989, and results will be cross-checked with spin-tracking simulations. In E821 the pitch correction was approximately $300 \mathrm{ppb}$ on $\omega_{a}$ [36, and the scale for the E989 correction is about half that, Section 5.5.8.

\subsection{Run 1 in E989}

Run 1 for E989 was conducted in the first half of 2018. Production data were gathered from March 22nd through June 29th. Because of accelerator, experimental, and practical concerns common to the early stages of production running, production data taking was interrupted at various dates. Due to hardware issues both kicker and quadrupole voltages were originally lowered from their design values. Various voltage set points for both systems were identified and used in separate periods of the data taking, depending on the stabilities of the systems. The primary precession frequency 
analysis datasets gathered by E989 and their associated running parameters are shown in Table 2.3. Other datasets were gathered but they were not used to calculate the precession frequency in this analysi: 5 .

Due to the lower kicker voltages, the muon beam was stored on a central radius $\sim 7 \mathrm{~mm}$ offset from the central orbit of the storage ring. The associated number of stored muons per fill for Run 1 as a result of the lower kicker voltage was $\mathcal{O}(4,000)$, down from the $\mathcal{O}(10,000)$ design goal. The chosen quadrupole voltages were 18.3 and $20.4 \mathrm{kV}$, corresponding to $n$ values of 0.108 and 0.120 respectively 65 . During Run 1 it was discovered that some of the quadrupole resistors were damaged. They had longer RC time constants such that the quadrupole voltages had not reached nominal values at the beginning of the designated analysis portion of the data at $30 \mu \mathrm{s}$, and were still changing over the course of a fill, see Figure $2 \cdot 20$. The muon beam was seen to move as a function of time in-fill as a consequence of this, beyond the ordinary CBO motion of the beam. See Section 4.4 for a summary of the muon beam characteristics for Run 1.

All data listed in Table 2.3 were quality checked. If run conditions were found to be unsatisfactory, the associated data was flagged and ignored in the analysis. A summary of the data quality control procedure is given in Reference [66], and the exact data quality parameters for the $60 \mathrm{~h}$ dataset are detailed in Reference [67]. The total number of detected positrons above threshold that passed the data quality control was approximately $1 \times 10^{10}$, corresponding to a statistical error on $\omega_{a}$ of $\sim 400 \mathrm{ppb}$, as calculated with Equation 2.16.

\footnotetext{
${ }^{5}$ Specifically it is expected to include the LowKick dataset in future analyses, which has approximately $80 \%$ of the statistics of the $60 \mathrm{~h}$ dataset.

${ }^{6}$ The associated betatron wavelengths are 1.06 and 3.04 times the circumference of the storage ring respectively.
} 


\begin{tabular}{|c|c|c|c|c|c|}
\hline \multicolumn{6}{|c|}{ Run 1 Precession Frequency Analysis Datasets } \\
\hline Name & Date Acquired & Number $e^{+}>E_{\mathrm{Th}}$ & Quad Voltage $(\mathrm{kV})$ & $n$ Value & Kicker Voltage $(\mathrm{kV})$ \\
\hline $60 \mathrm{~h}$ & $4 / 22 / 18-4 / 25 / 18$ & $9.34 \times 10^{8}$ & 18.3 & 0.108 & $128-132$ \\
\hline HighKick & $4 / 26 / 18-5 / 02 / 18$ & $8.70 \times 10^{8}$ & 20.4 & 0.120 & $136-138$ \\
\hline $9 \mathrm{~d}$ & $5 / 04 / 18-5 / 12 / 18$ & $2.13 \times 10^{9}$ & 20.4 & 0.120 & $128-132$ \\
\hline Endgame & $6 / 06 / 18-6 / 29 / 18$ & $4.10 \times 10^{9}$ & 18.3 & 0.108 & $122-127$ \\
\hline Total $\mathrm{Pc}$ & ns Above Threshold & $8.03 \times 10^{9}$ & & & \\
\hline
\end{tabular}

Table 2.3: The primary datasets acquired during Run 1 of E989 in 2018 and their associated parameters [68]. Other datasets were gathered but they were not used in the calculation of the precession frequency in this analysis. $E_{\mathrm{Th}}=1.7 \mathrm{GeV}$. 


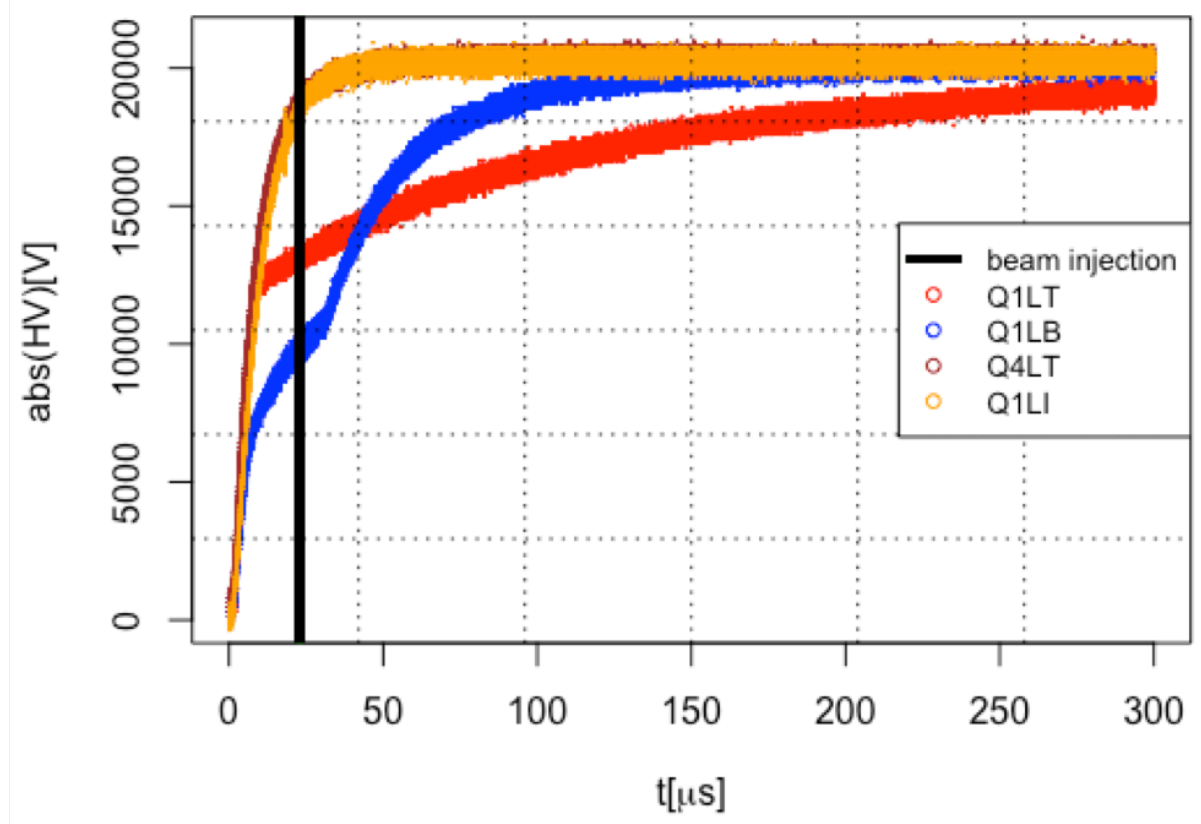

Figure 2.20: Traces for some quadrupole plate potentials as a function of time. The different plates are identified in the legend, where for instance Q1LT stands for the top long plate of quadrupole 1. The muon beam is injected into the ring at the black line and the scraping procedure occurs until the quadrupole voltages reach their design values, in this case $20.4 \mathrm{kV}$. As shown some of the high voltage traces do not behave in a smooth and fast exponential manner, due to damaged quadrupole resistors. The traces in orange and brown are for good quadrupole resistors, while the traces in red and blue show the results from damaged quadrupole resistors. The kink in blue is the switch from the scraping procedure to storage. 


\section{Chapter 3}

\section{Detector Systems}

There is a variety of different detector systems used in E989. The primary detectors are the calorimeters which measure the $\omega_{a}$ signal. In support of that measurement there are several auxiliary systems used for monitoring injection and beam dynamics. These include the T0, IBMS, and fiber harps. There is also a straw tracking detector which measures decay positron trajectories which can be related to muon beam dynamics and calorimeter measurements. Each of these systems will be described in the following sections.

\subsection{Auxiliary detectors}

\subsubsection{T0}

A reference time must be chosen so as to align all different detector systems in time. Without this functionality, analyzing the data from different systems and comparing them would be impossible. Similarly, decay positron spectra must be aligned in phase from fill to fill, otherwise the $\omega_{a}$ signal would wash out. To do this a "T0" counter is placed on the outside of the ring just before the inflector. It is made up of a scintillating paddle connected to two photo-multiplier tubes (PMTs) [69, 70]. See Figure $3 \cdot 1 \mathrm{a}$. PMT A has a $1 \%$ neutral density filter which results in a low photoelectron statistics measurement, and acts primarily as a timing measurement. PMT $\mathrm{B}$ has a $10 \%$ neutral density filter which results in a higher statistics measurement, and acts as a shape counter and proxy for fill intensity. (In general the signals are 


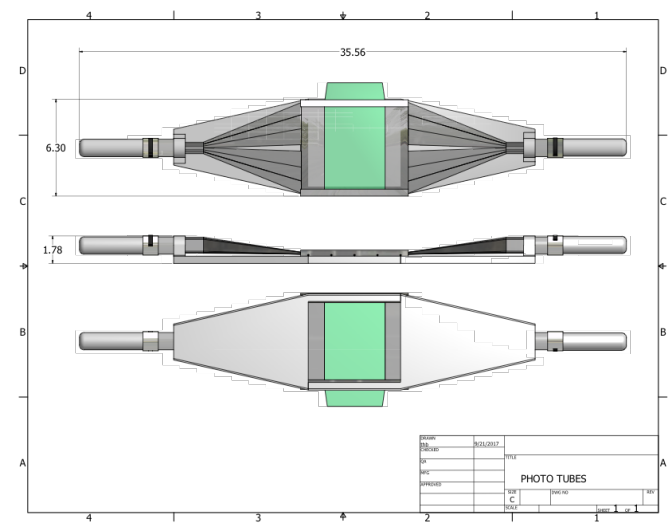

(a) The T0 counter is made up of a scintillator in the middle shown in green, which connects with light guides to PMTs on the left and right.

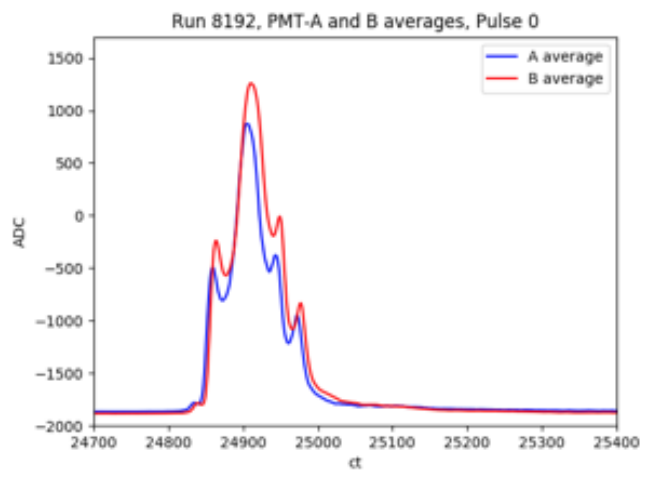

(b) Time profiles for the two PMTs in the T0 counter for one of the eight bunches. Each profile is an average of 100 such profiles. The $\mathrm{x}$ axis is in clock ticks, where $1 \mathrm{ct}=1.25 \mathrm{~ns}$.

Figure 3·1: A model of the T0 detector (left) and a sample of the signal it measures every fill (right).

very similar.) Together they provide a measure of the injected beam profile in time, from which the timing alignments can be made. Some measured T0 pulses are shown in Figure $3 \cdot 1 \mathrm{~b}$, The shape of the incoming pulses has a somewhat trident-like shape, with a very large spike in the middle of the time profile and two spikes on the outside edges. This shape owes itself to the accelerator complex, and varies somewhat from bunch to bunch.

\subsubsection{Inflector Beam Monitoring System}

While the T0 provides timing and intensity measurements, the inflector beam monitoring system (IBMS) provides measurements of the beam position properties as it passes through the inflector. This is useful because the injection aperture is so tight, and incoming beam parameters are tightly constrained. The IBMS system serves to provide a direct diagnostic handle on the phase space matching between the last accelerator components and the storage ring, helping to optimize the number of stored 

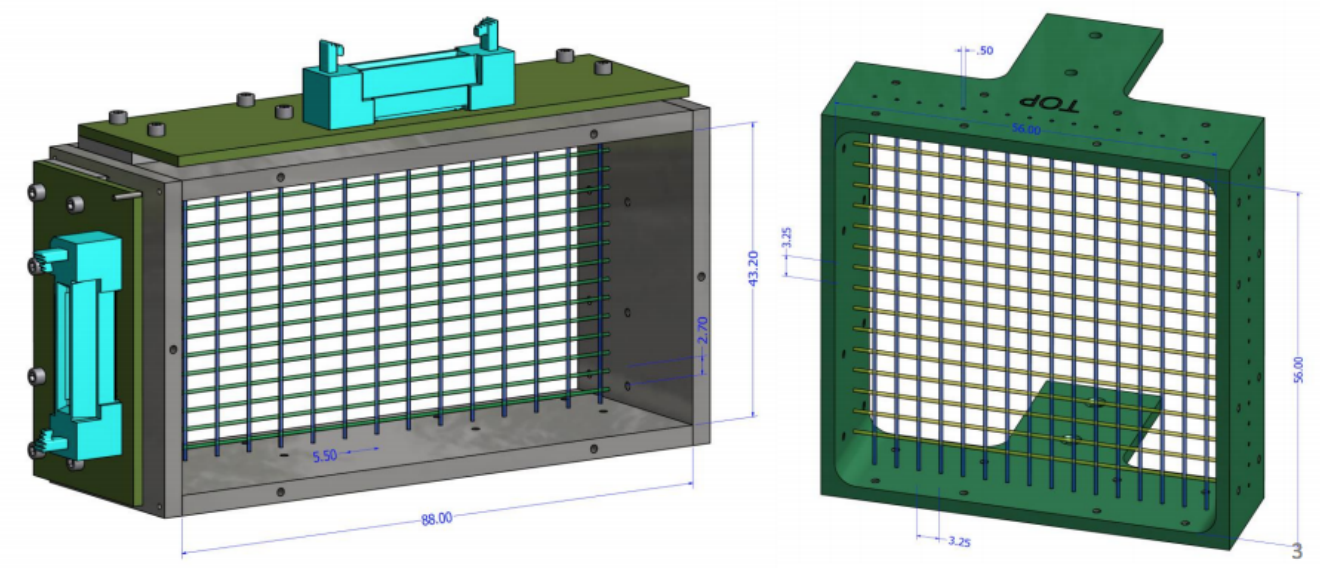

Figure $3 \cdot 2$ : Models of the IBMS 1 and 2 detectors. Scintillating fibers form an array which detect particles as they pass through them.

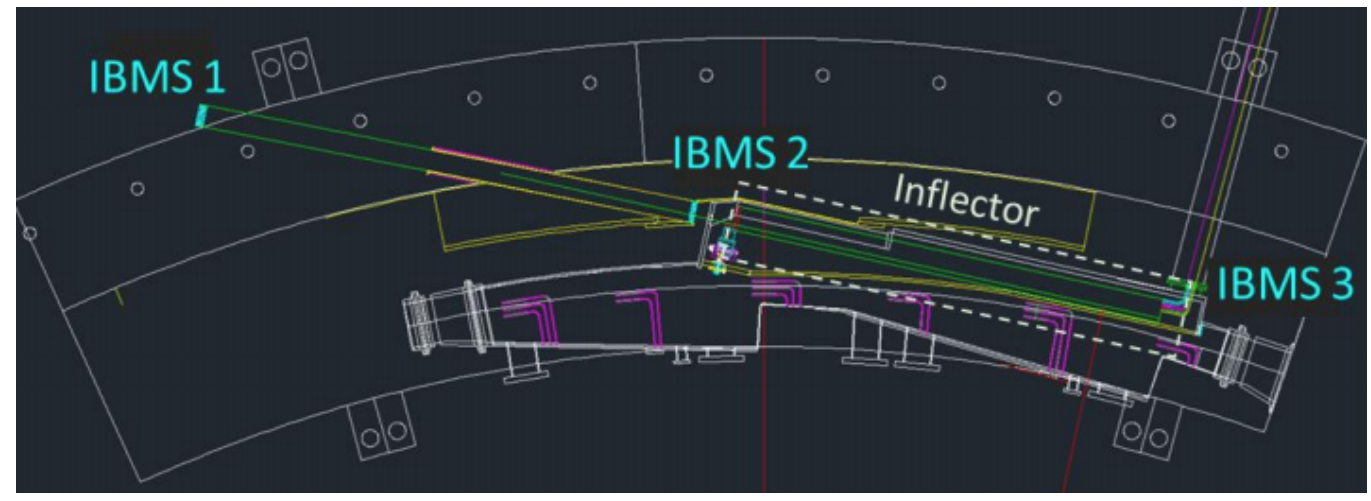

Figure 3.3: The positions of IBMS 1, 2, and the planned 3rd device are shown with respect to the vacuum chamber and inflector.

muons per fill [71]. The IBMS is made up of two scintillating fiber detectors, shown in Figure $3 \cdot 2$. These devices are placed at the outside of the magnet yoke before injection into the back hole of the magnet, and at the entrance to the inflector 72 . A third device is planned to be at or near the downstream end of the inflector. See Figure $3 \cdot 3$.

\subsubsection{Fiber harps}

The last auxiliary detector is the "fiber harp" system. It is made up of four scintillating fiber detectors that serve to measure the beam intensity as a function of time 


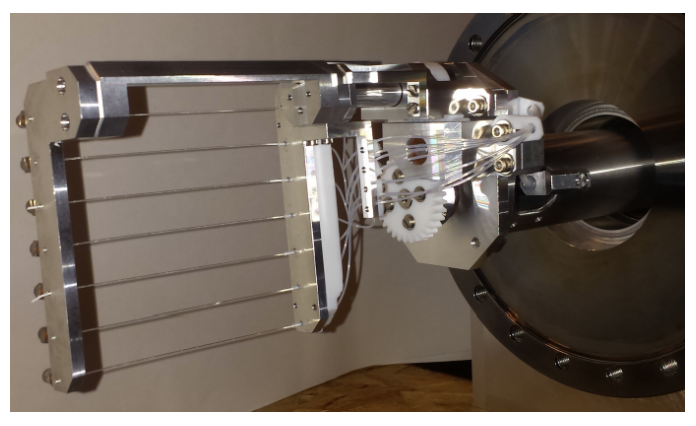

(a) Picture of one of the fiber harps. A row of seven scintillating fibers measures the beam intensity as a function of time at vertical or horizontal positions depending on which fiber harp is inserted.

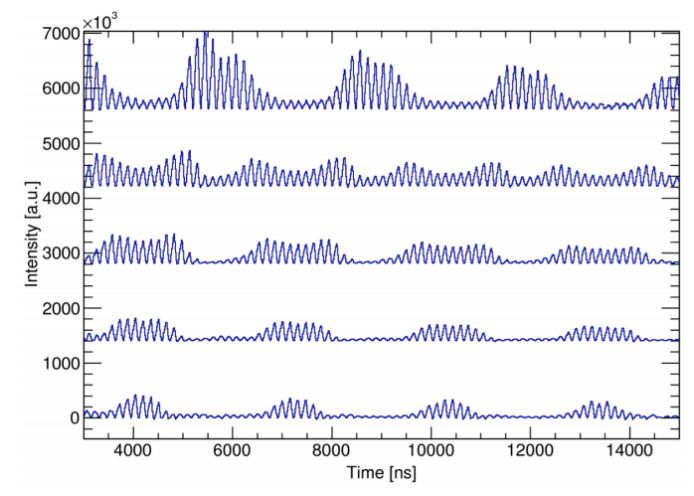

(b) Shown are fiber harp beam intensity measurements for a horizontal fiber harp. Each spectra is from a single fiber, with the spectra at the top being at the greatest radial position. The fast oscillations of the cyclotron frequency can be seen along with the slower oscillations of the CBO.

Figure 3·4: Fiber harp detector (left) and a sample of fiber harp measurements (right).

and position within the storage ring [73]. Two of the detectors measure the radial components of the beam, and two measure the vertical components. Each pair of fiber harps are located $180^{\circ}$ and $270^{\circ}$ in azimuth clockwise from the inflector. One of these devices is shown in Figure $3 \cdot 4$ a. The fiber can measure beam properties throughout the fill, providing a diagnostic tool which is sensitive to the scraping procedure and the CBO properties of the beam. An example of fiber harp measurements is shown in Figure $3 \cdot 4 \mathrm{~b}$. However, because the fiber harps are a destructive measurement of the beam due to multiple scattering in the fibers, the system was designed to be retractable. They are inserted during special diagnostic or systematic runs, and are pulled out of the beam path during production data taking. 


\subsection{Calorimeters}

Electromagnetic calorimeters are the primary detector in the E989 experiment, responsible for the $\omega_{a}$ measurement. They measure arrival times and energies of the decay positrons as they curl inward from the storage region.

\subsubsection{Requirements and systematic effects}

In order to determine $\omega_{a}$ to the precision goal, there are specific requirements on the performance of the calorimeters. First, they must have a relative energy resolution of better than $5 \%$ at $2 \mathrm{GeV}$, in order for proper event selection above threshold [54]. The energy resolution requirement is modest but not strict because the important observable is the number of detected positrons above some energy threshold, where the optimal energy threshold can be determined empirically.

Second, they must have a timing resolution of better than $100 \mathrm{ps}$ for positrons with energy greater than $100 \mathrm{MeV}$ [54]. The calorimeters must be able to resolve multiple incoming hits through temporal or spatial separation at 100\% efficiency for time separations of greater than $5 \mathrm{~ns}$, or $66 \%$ of hits for time separations less than $5 \mathrm{~ns}$, in order to reduce the pileup systematic effect. Pileup is the term used for when multiple particles impact the detector within the dead time of the detector such that they are registered as a single hit. Unresolved pileup means that the number of detected positrons above threshold is mis-measured. Since pileup is a time-dependent effect, where pileup decays with a lifetime approximately equal to half the muon lifetime, this leads to a mis-measurement of $\omega_{a}$, see Section 5.2.1. The requirements stated here assist in reducing the pileup systematic error below a target goal of $40 \mathrm{ppb}$.

Third, the energy response of calorimeters for measured hits must be stable to $<0.1 \%$ over a fill $(700 \mu \mathrm{s})$ [54. The long term energy response stability over a time period of order seconds must be $<1 \%$. The energy response of a detector as a function 
of time is typically referred to as the gain of the detector, where technically the gain refers to the amount of current output per detected hit. The gain depends on the temperature stability and hit rate. After a hit, the measured energy fraction of a following hit drops sharply and then rises exponentially back to one. This occurs as a result of pixels in the detector being unable to fire consecutively within short time frames, $\mathcal{O}(\mathrm{ns})$, and because of charge depletion in the calorimeter system's electronics capacitive components, $\mathcal{O}(\mu \mathrm{s})$. The former is referred to as the short-term double pulse (STDP) effect [74. The latter is referred to as the in-fill gain (IFG) effect 75], where the effect is due to the high rate of incident particles at injection combined with secondaries which overcomes the recovery response of the electronics at early times in the fill 1 . Hits with mis-measured energies due to these effects can thus be excluded from the fitted histogram if their energies drop below threshold, leading to another systematic effect and subsequent error in the $\omega_{a}$ measurement. (Temperature changes vary over time scales greater than a fill, so they don't contribute to the systematic error.) The requirements stated here, along with the use of a laser calibration system (Section 3.2.3), assist in reducing the gain systematic error below a target goal of $20 \mathrm{ppb}$.

\subsubsection{Hardware}

There are 24 calorimeters located symmetrically around the inside of the ring placed flush to the vacuum chamber wall, as shown in Figure 3.5a, Indeed the shape of the vacuum chambers were designed so as to reduce multiple scattering of the decay positrons before entering the calorimeters. Each calorimeter sits on a board extending out from a cart which contains the electronics that power the calorimeters and read out the data. The carts help to relocate magnetic materials away from the field region

\footnotetext{
${ }^{1}$ The large of amount of particles and secondaries at the beginning of a fill is referred to as the 'flash'.
} 


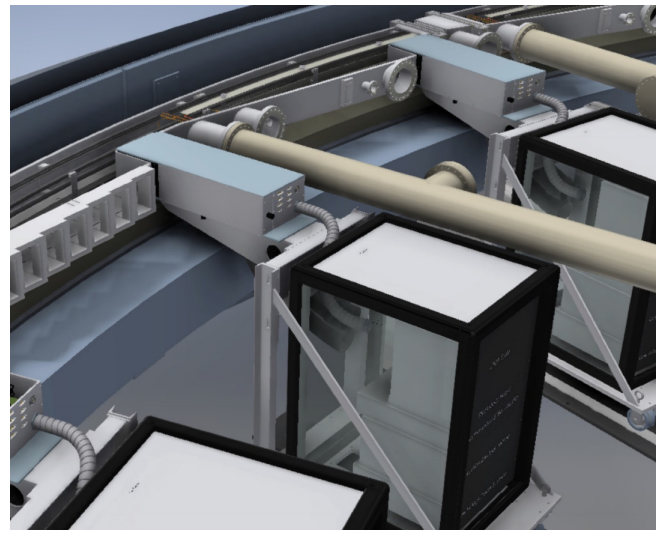

(a) Close up view of a model of the storage ring and calorimeter detector systems. In black are carts which house the electronics for the calorimeters. They hold up a board upon which the calorimeter rests, identified by the long gray box. The calorimeter is pushed flush to the vacuum chamber on the left.

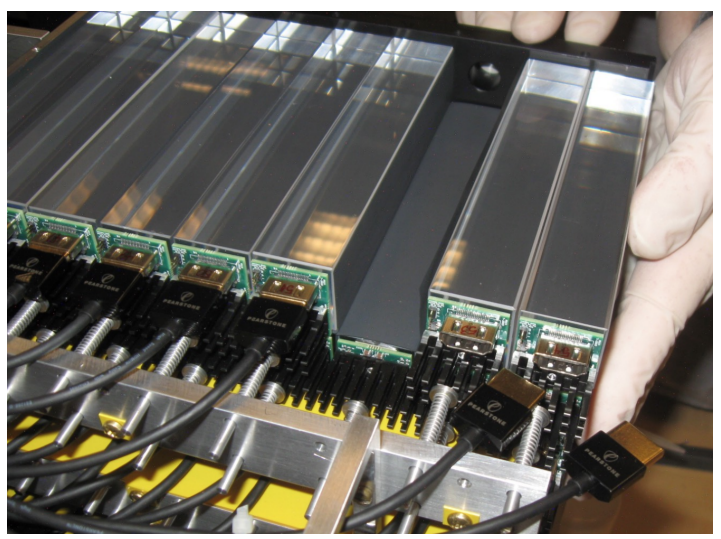

(b) Shown is the backside of a calorimeter. The clear blocks are $\mathrm{PbF}_{2}$ crystals, each of which has a SiPM mounted onto the end in order to collect emitted Cerenkov light from incident particles.

Figure 3.5: Calorimeter detector systems relative to the vacuum chamber (left) and an individual calorimeter (right).

to avoid perturbing the magnetic field, and provide easy access to the electronics while removing them from the positron decay path region.

Each calorimeter consists of 54 channels of $\mathrm{PbF}_{2}$ crystals in a 6 high by 9 wide array, for a total of 1296 channels. Each crystal is $2.5 \times 2.5 \times 14 \mathrm{~cm}^{3}$ and is wrapped in black Tedlar@ foil. The $\mathrm{PbF}_{2}$ material has an index of refraction of 1.8 , and emits Cerenkov light as incident positrons with energy greater than $100 \mathrm{keV}$ pass through the crystals [76]. Cerenkov light is naturally fast which improves the timing resolution of the incoming hits. The high density of the $\mathrm{PbF}_{2}\left(7.77 \mathrm{~g} / \mathrm{cm}^{3}\right)$ gives it a short radiation length $(9.3 \mathrm{~mm})$, such that the energy deposition from the incident positrons is nearly $100 \%$ over the length of the crystal. The black foil is used to eliminate light transmission between crystals to improve position reconstruction, as well as reduce internal reflection to reduce pulse width and improve timing resolution [77]. The 
energy deposition from the incident positrons is typically restricted to only one or two crystals. The segmentation of the calorimeter combined with the black wrapping helps the spatial and temporal resolution of the detected pulses. See Figure $3 \cdot 5 \mathrm{~b}$ for a picture of the calorimeter crystals.

Each crystal is paired with a large area silicon photo-multiplier ( $\mathrm{SiPM}$ ) sensor which detects the emitted Cerenkov light. SiPMs are compact, operable in high magnetic field regions, have very linear responses for operation at $\mathrm{MHz}$ rates, are suited to measuring Cerenkov light due to their high photo-detection efficiency in the wavelengths of interest, and have a high degree of gain stability [77]. The SiPMs used are designed by Hamamatsu and detailed in Reference [77]. They sit on printed circuitry boards (PCBs) devoid of magnetic materials, which are designed to preserve the fast SiPM pulse shape. The combined properties of the chosen SiPMs, their electronic boards, and the $\mathrm{PbF}_{2}$ crystals results in an energy resolution of (4.6 \pm $0.3) \% / \sqrt{E / \mathrm{GeV}}$, a timing resolution of $20 \mathrm{ps}$, and a pulse width of $5 \mathrm{~ns}$, satisfying the stated requirements earlier [76, 77]. Details of the pulse fitting algorithms are shared in Section 5.1. The SiPMs can be seen in Figure 3.5b. Finally, 12-bit waveform digitizers (WFDs) sample each SiPM channel at a rate of 800 mega-samples per second with a $1 \mathrm{~Gb}$ memory buffer and the data are transferred to a bank of GPU processors for on-line data processing [78]. The timing resolution of these WFDs is $<50$ ps for most pulse amplitudes.

\subsubsection{Laser calibration system}

In order to satisfy the gain requirements of the calorimeter detectors, a laser calibration system is used. This system monitors the SiPM responses over short and long time scales to $<0.04 \%[79$. The system consists of six different lasers and a suite of optical devices. The light from the six lasers is piped through optical fibers to a board mounted on the front face of each calorimeter. This board contains right-angle 
prisms which deflect the laser pulses directly into each calorimeter crystal, for all 1296 channels. The lasers can be pulsed at various intensities, both in-fill to monitor the STDP or IFG effects, and out-of-fill to monitor for long term drifts due to changing temperatures. The SiPM measured response is compared to local known source monitors in order to calibrate the SiPM energy response. Corrections to the gain of the calorimeters can thus be determined and applied to the hit energies. Simultaneously the laser system allows for measuring the timing resolution of the SiPMs, and in general performing diagnostic tests with the calorimeter. Lastly, the laser system is used to time align the different calorimeter channels by outputting a sync pulse to each channel at the beginning of every fill.

\subsection{Straw trackers}

As described in Section 2.6, the muon beam moves as a whole within the storage ring. As explained in Section 2.2, the muon beam distribution ties into the measurement of the average magnetic field. The primary detector system used to measure this behavior and determine the muon beam distribution is the "straw tracker" system. The straw trackers characterize the beam in a non-destructive fashion by measuring decay positron trajectories and extrapolating them back into the storage ring. They serve to

provide the direct measurement of the pitch correction as described in Section 2.7.2, determine the momentum distribution of the beam, and characterize parameters of the $\mathrm{CBO}$ which tie into the calorimeter $\omega_{a}$ analysis. Decay positron trajectories can also be extrapolated forwards into the calorimeter, in order to cross-check calorimeter measurements to help resolve pileup. Finally, the trackers also serve as a measuring device to search for a possible muon electric dipole moment. The existence of such a thing would tilt the precession plane of the muons and subsequent decay positron trajectories which the trackers are sensitive to. Details of the beginnings of such a search 
are given in Reference [80]. The details of the track reconstruction and analysis will be given in Chapter 4. Here will be given a description of the detectors themselves.

In general, straw tracking detectors work by measuring hits in gas filled straws [81. Each straw is made up of a cylindrical piece of thin material, typically Mylar with a conductive aluminum coating, and contains a wire at the center of the straw held at high voltage, $\mathcal{O}(1000 \mathrm{~V})$. The minimal amount of material in straw trackers serves to reduce multiple scattering of incident particles, and was the reason a straw tracker system was chosen over other types of trackers. Fast moving charged particles ionize the gas as they pass through it, and the resulting ions are drawn to the wire and straw surface (positive and negative charges respectively). As the ions move to the wire, they enter a high electric field region that causes them to speed up, hit other gas molecules, and create more ions. This produces an avalanche gain effect which amplifies the original signal. Once the ions reach the wire and straw surface, an electrical signal is read out telling what the drift time of the ions was, which can be related to the radius or distance of closest approach (DCA) at which the particle passed relative to the wire. The straws therefore measure drift circles in a plane perpendicular to their physical orientation. The calculations of these DCA's and their errors is done using a simulation program for gaseous detectors called Garfield 82 , 83. By combining several such measurements in time and space, tracks of incident particles can be reconstructed. See Figure $3 \cdot 6$.

The straws used in E989 are $5 \mathrm{~mm}$ in diameter and contain a 50:50 mixture of argon-ethane gas [84]. The argon component serves as the gas to be ionized. The fast moving ions near the wire in addition to producing more ions, will incite excited states of the gas, which emit photons when de-excited. These photons can restart the whole ionization and avalanche process over again as they can escape the avalanche region, leading to a break-down of the system. The ethane therefore serves to absorb the 

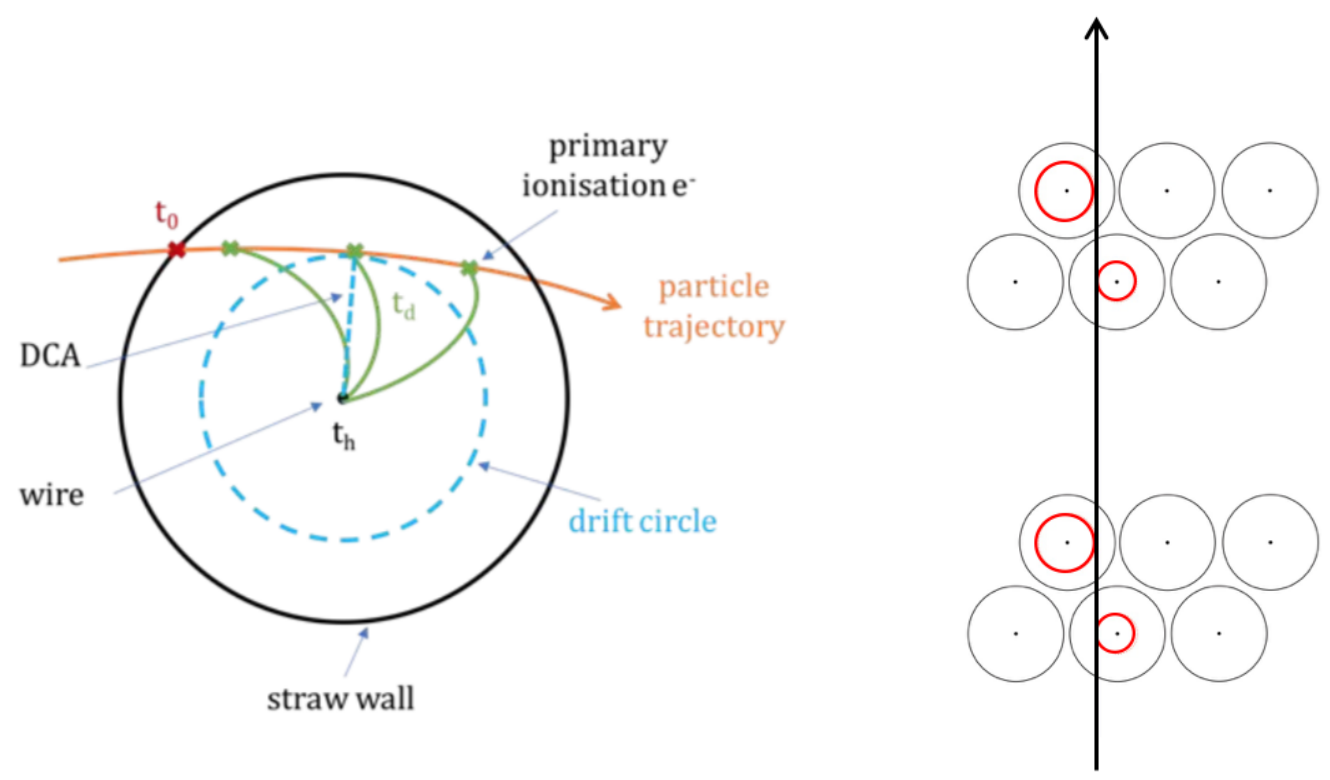

Figure 3·6: Diagrams showing the determination of a drift time $t_{d}$ and hit time $t_{h}$ from an incident ionizing particle (left) and the combination of several such hits to produce a track (right). Diagram on the left courtesy of S. Charity.

photons with its large number of molecular degrees of freedom and photo-absorption characteristics [84]. The wire has a radius of $25 \mu \mathrm{m}$ and is made up of gold-plated tungsten. The Mylar walls have a width of $15 \mu \mathrm{m}$ and are wound in a double spiral shape in order to improve the electrostatic shielding of the wire and reduce the gas leak rate of the straws 84 . The DCA resolution of hits within the straws is approximately $120-150 \mu \mathrm{m}$.

A tracker module is shown in Figure 3.7. Each tracker module consists of 4 layers of 32 straws each with stereo angles of $\pm 7.5^{\circ}$, for a total of 128 straws per module. The first two straw layers are designated as " $\mathrm{U}$ " layers, and are oriented with the tops of the straws at greater radial positions than the bottoms. The second two layers are designated as "V" layers, and are oriented with the bottoms of the straws at greater radial positions than the tops. Both $\mathrm{U}$ or $\mathrm{V}$ layers are collectively referred to as the $\mathrm{U}$ and $\mathrm{V}$ views of the module respectively. See Figure $3 \cdot 8$. The two layers in 


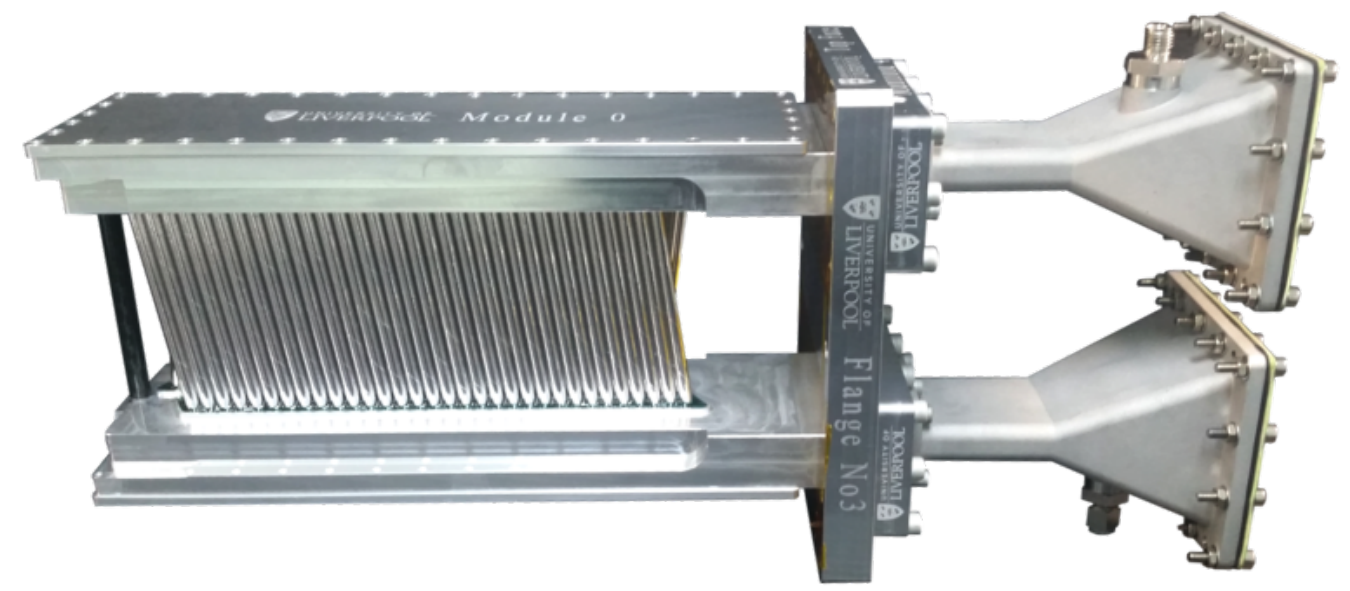

Figure 3·7: A straw tracker module. The first layer of silver Mylar straws with a stereo angle of $7.5^{\circ}$ can be seen, with the other three straw layers hiding behind it. In black on the left is the carbon fiber post which holds the end of the module in a fixed position. Electronics live in the top and bottom manifolds above and below the straws, and cables connect from those electronics through small apertures to external electronics which plug in on the right.

each view are shifted half a straw diameter horizontally from one another in order to help resolve which side of the wire any incident particles passed, Section 4.2.6. The two types of layers are non-parallel to each other in order to resolve incident tracks in $2 \mathrm{D}$ space. The small stereo angle both improves the straw measurement area as electronics can be kept out of the positron decay path region, and improves the radial momentum resolution of the fitted tracks, since both views measure mostly in the horizontal plane. The active straw measurement area is approximately $10 \mathrm{~cm}$ high by $20 \mathrm{~cm}$ wide. A carbon fiber post sits at the outside end of the module to provide structural rigidity to the module, and keep the straw wires under tension.

There are two tracker stations, each consisting of eight tracker modules. The two stations are located at positions in front of calorimeters 13 and 19, or at approximately $180^{\circ}$ and $270^{\circ}$ clockwise from the inflector. These are designated as Tracker 

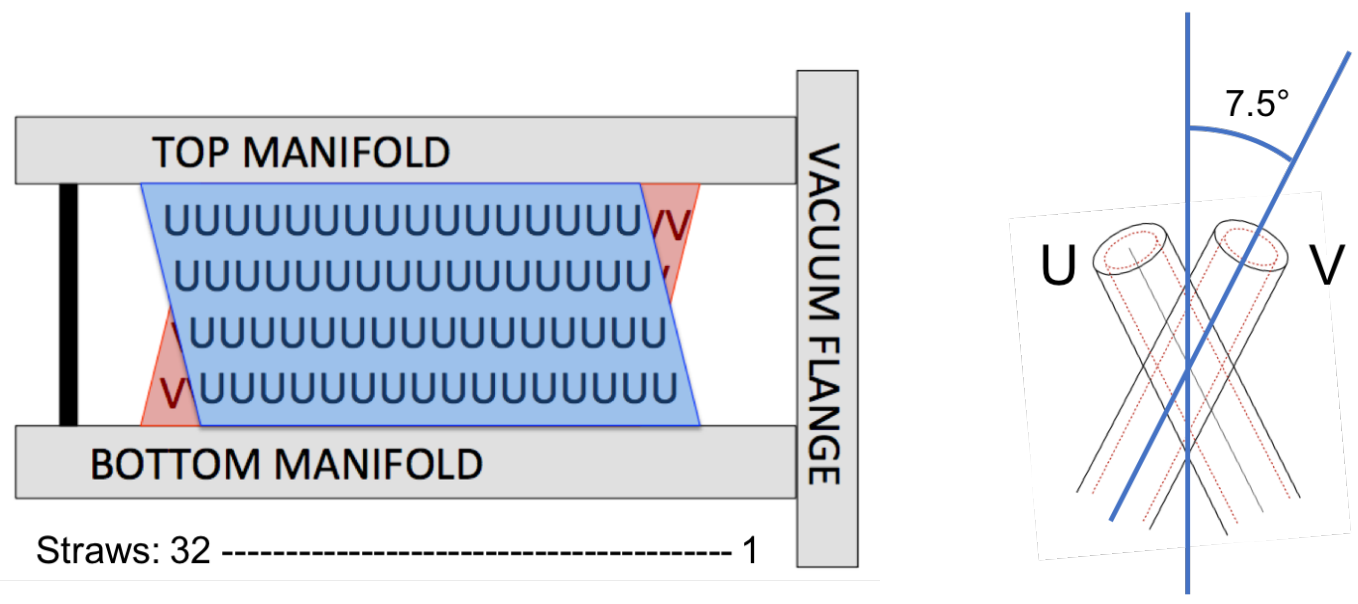

Figure 3.8: Orientation of $\mathrm{U}$ and $\mathrm{V}$ straws in the tracker module.

Station 12 and Tracker Station 18 respectively, where the numbers come from which vacuum chamber the trackers reside in. There is also a third station just after the inflector, which currently sits empty of any tracker modules. The straw tracker modules sit inside the vacuum chamber in specially modified vacuum chamber sections in a staircase-like pattern that follows the curvature of the ring. See Figure 3.9 and Figure $3 \cdot 10$. The number of modules and their orientation in each station was chosen to provide a long measurement arm for precise momentum measurement of the incident tracks. The modules sit inside the vacuum in order to eliminate multiple scattering in air and produce better reconstructed tracks. Due to their proximity to the storage region, the tracker modules are located in a region of high field non-uniformity. Though the acceptances between the trackers and calorimeters are not identical, their close proximity facilitates comparison between the two measurement devices.

\subsubsection{Tracker readout electronics}

The readout electronics for the system are split into two groups, the front-end and back-end. The front-end electronics were built and tested in the Boston University Electronic Design Facility. They start with ASDQ (Amplifier Shaper Discriminator with charge $(\mathrm{Q})$ ) chips on boards that plug down directly onto the straws and pro- 


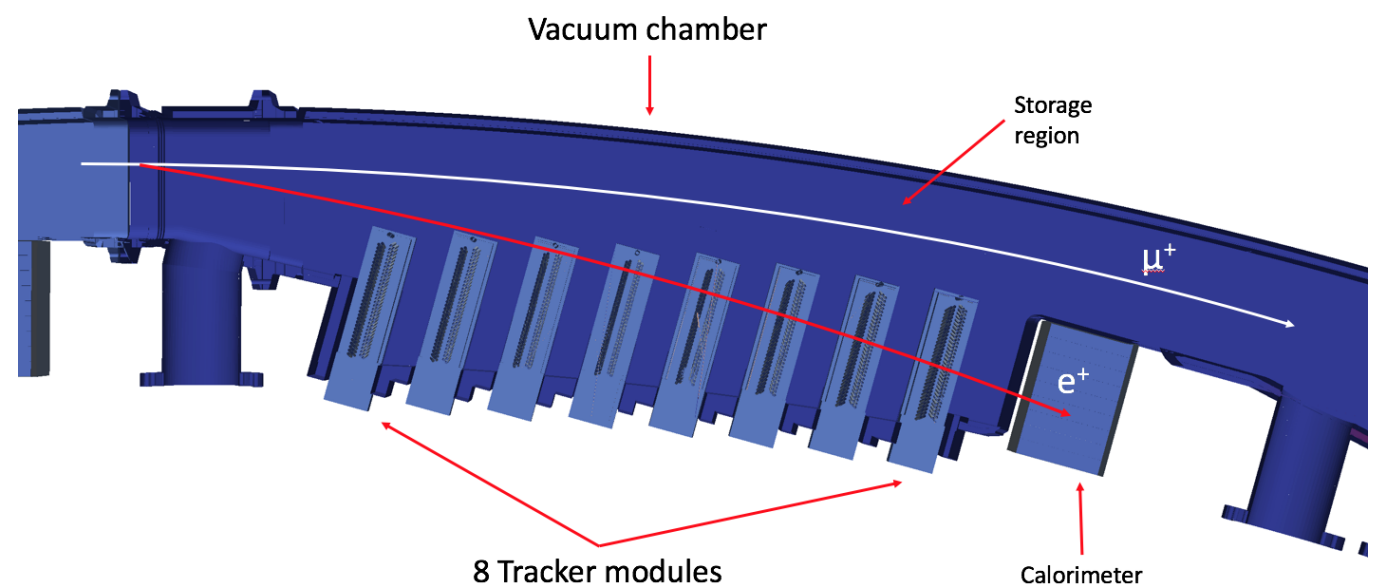

Figure 3.9: Birds eye view of a model of a vacuum chamber containing a tracker station, and the associated calorimeter. Each tracking station consists of eight tracker modules.

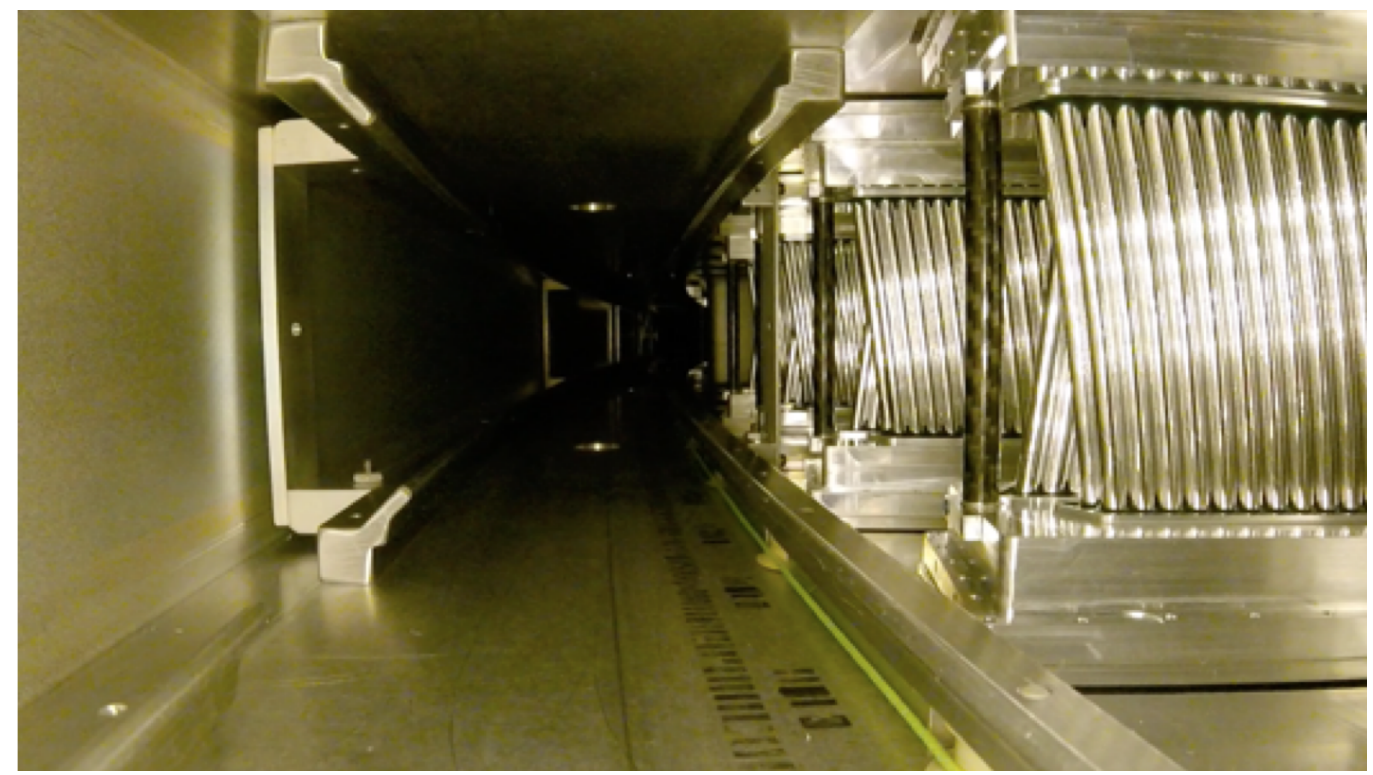

Figure 3.10: View of the tracker modules from within the storage ring. Muons move forward into the page, some of which decay to positrons which then pass through the trackers arrayed on the right. The carbon fiber post for each module can also be seen. 
vide the main signal, Figure 3·11a, Each ASDQ plugs onto sixteen straws. These ASDQ boards are application specific integrated circuits (ASICs) which read out the signal from one end of the straws and shape and discriminate that signal, as well as provide some baseline restoration and tail cancellation. The ASDQs and their associated components are contained within thin aluminum manifolds above and below the straws. The physical footprint of these boards and components was minimized in order to increase the straw measurement area. The signals from the ASDQs are passed through Flexi Cables to Time to Digital Converter (TDC) boards which time stamp the signals with 625 ps precision [84], Figure 3•11c. The Flexi Cables are flexible and thin such that the cables can be passed through the thin aperture shown on the right of Figure $3 \cdot 7$. High voltage is provided to the sense wires with high voltage boards, which pass the voltage through the ASDQs. A feedthrough board provides the interface between the TDCs and Flexi Cables, as well as the high voltage signal, Figure $3 \cdot 12$. They serve a dual purpose of providing the gas seal. Finally, there are logic boards that serve as the interface between the TDCs and the back-end electronics. They manage the clock and controls for the TDCs and store data onto FPGAs, which are piped out through a high throughput optical fiber connection. The logic boards, high voltage boards, and TDCs are all housed within an aluminum box which provides RF shielding, Figure $3 \cdot 13$. In each module there are eight ASDQs, four TDCs, two feedthrough boards, two high voltage boards, and two logic boards. An overview diagram of the front-end readout chain is shown in Figure $3 \cdot 14$. The back-end electronics consist of FC7s, one per tracker station, and a single AMC13 for all tracker stations. These modules provide clock and DAQ services to the whole tracker system, and ultimately pipe out the data to where it can be saved on disk. 

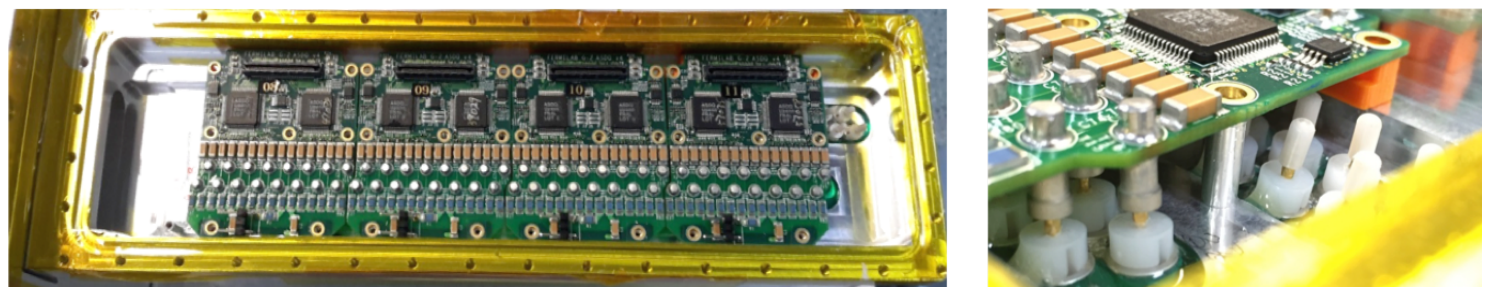

(a) ASDQs contained within the aluminum manifold (left) that plug down onto the straws (right).
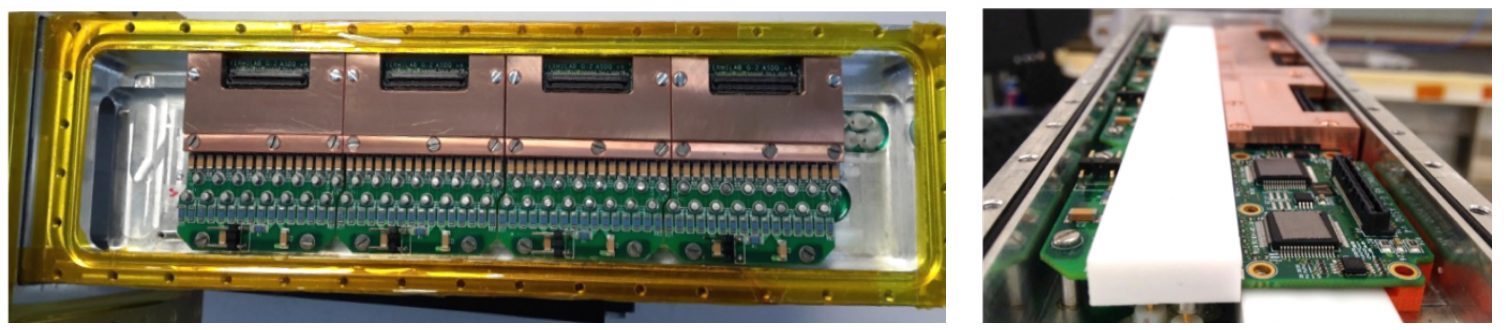

(b) Copper cooling bars (left) keep the ASDQs cool, and a high voltage spacer (right in white) keep the high voltage components isolated.
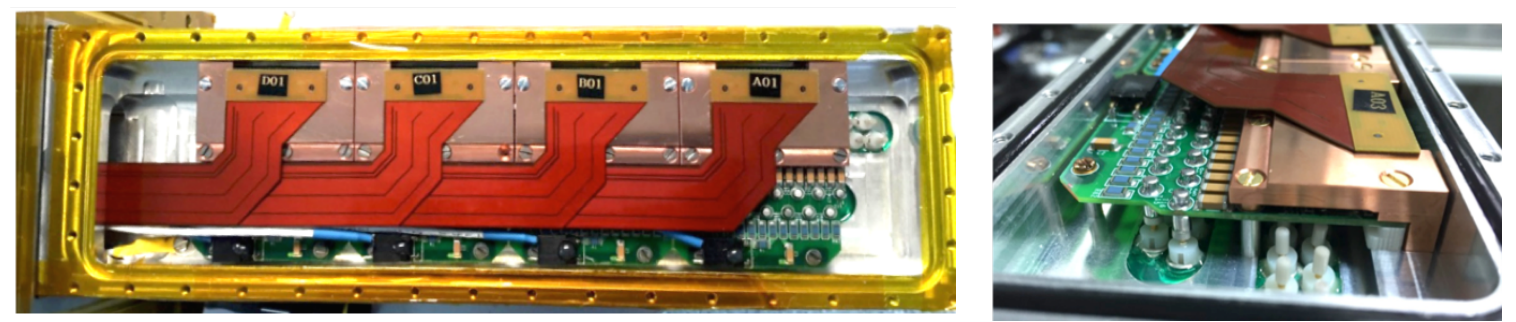

(c) Orange Flexi Cables plug down onto the ASDQ boards and run left through a small aperture in the tracking detector to the TDCs.

Figure 3.11: ASDQs and associated components which read out the signals from the straws. 

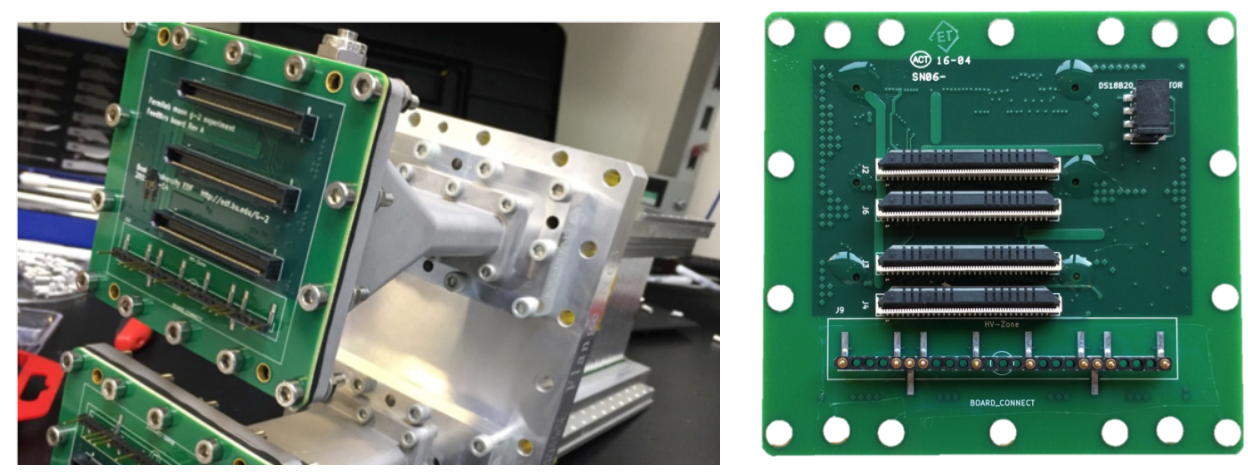

Figure 3·12: Tracker feedthrough board. The feedthrough board plugs onto the end of the tracker snout to form a gas seal (left). Outside of the gas seal, a logic board, two TDCs, and a high voltage board all plug into the feedthrough board. Flexi Cables plug into the back side of the feedthrough board (right).
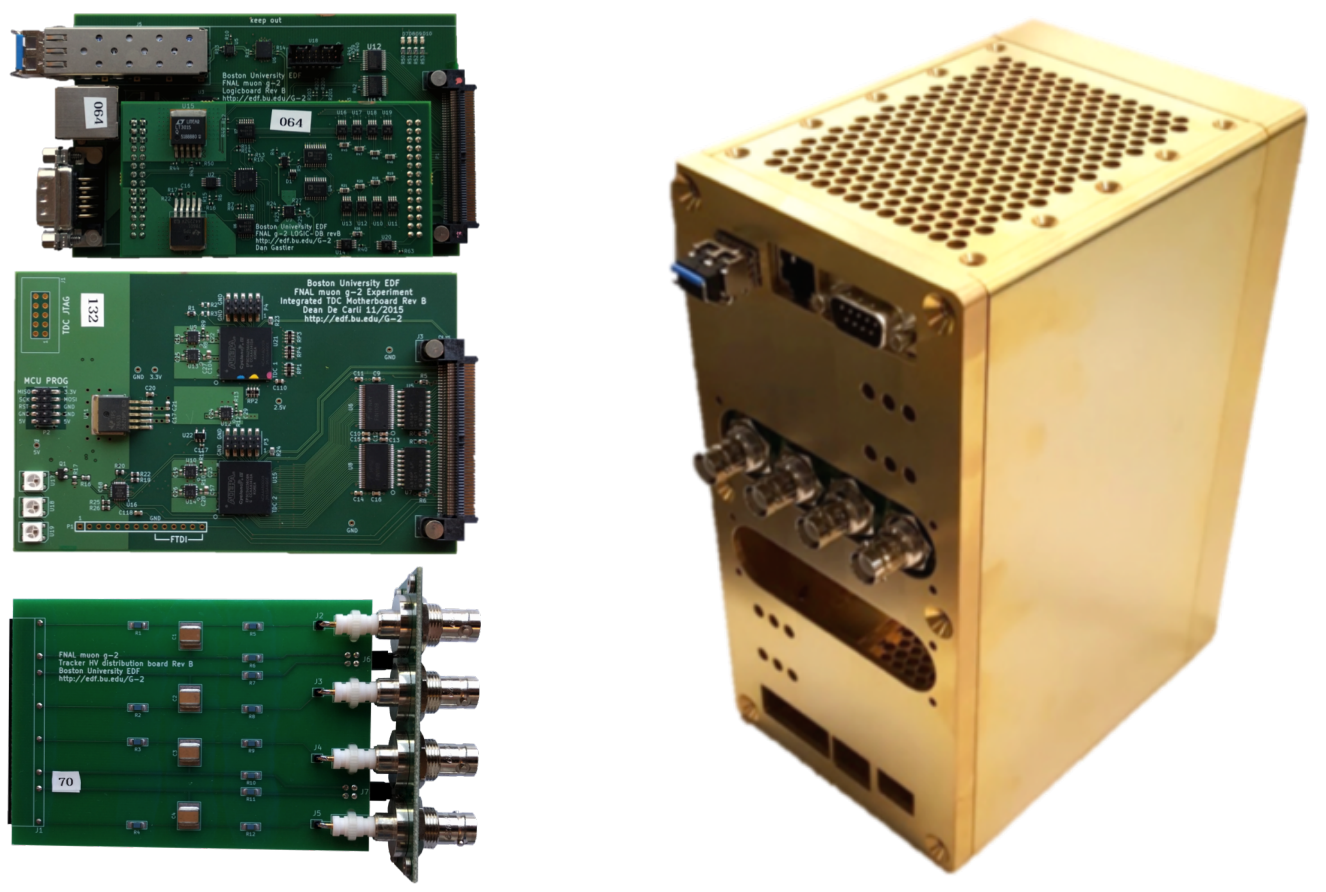

(a) A logic board (top), TDC (middle), and high voltage board (bottom).

(b) The aluminum housing which houses the front-end readout boards.

Figure 3.13: The front-end readout boards (left) and their housing (right). The logic and high voltage board connectors can be seen peeking out of their respective slots in the housing. 


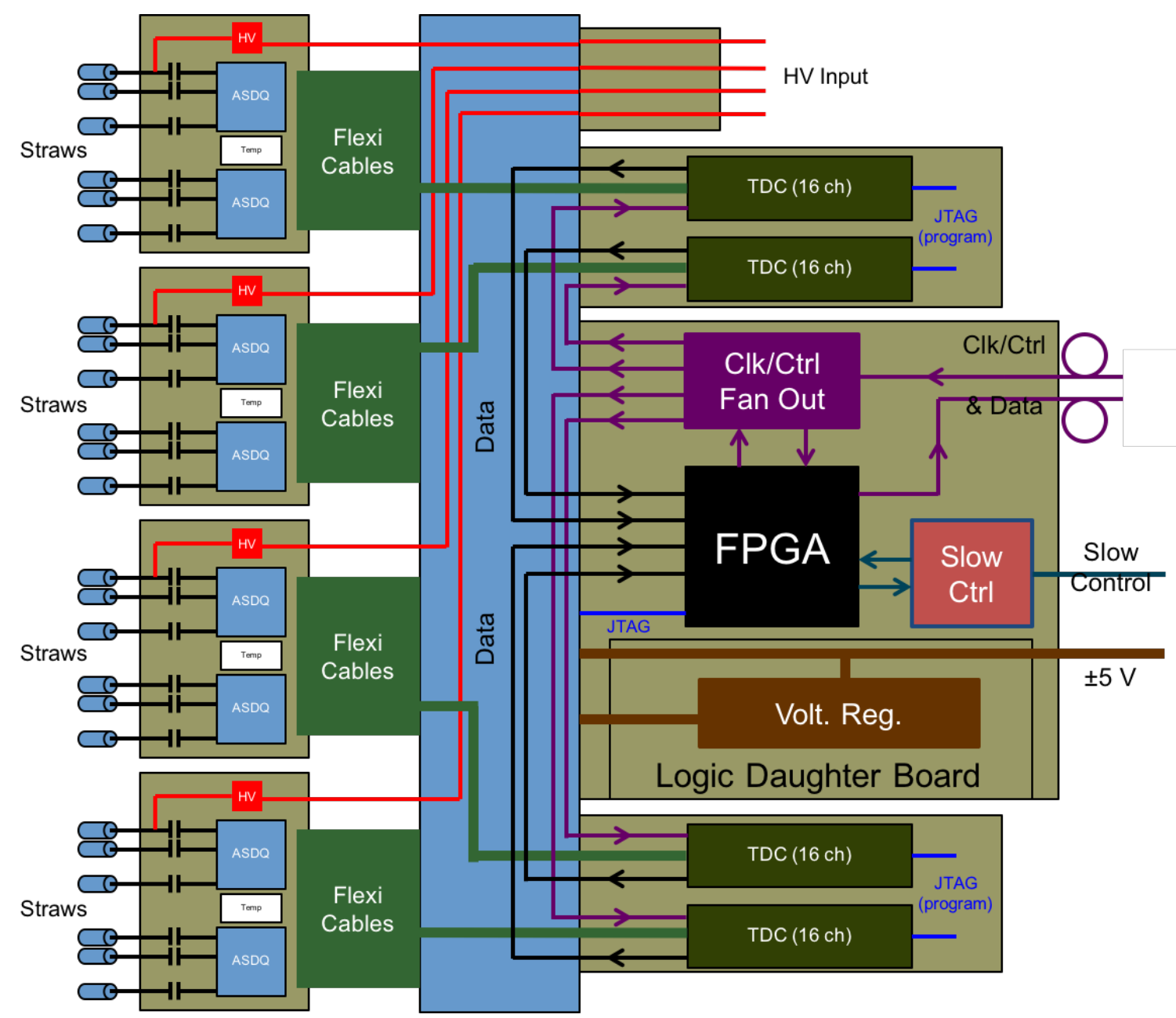

Figure 3.14: Front-end tracker electronics readout chain. Courtesy of James Mott. 


\section{Chapter 4}

\section{Track Reconstruction and Analysis}

The straw trackers are used to provide information about the muon beam, which is important for the calorimeter $\omega_{a}$ analysis, calculating the $\omega_{a}$ pitch correction, and determining the spatially weighted magnetic field seen by the muons. Section 3.3 described the physical characteristics of the straw trackers; this chapter focuses on the reconstruction of incident tracks. The track reconstruction is performed in three stages. First, individual hits in the tracker are grouped into tracks in the finding stage. Second, a best estimate trajectory is fit to grouped hits in the fitting stage. Third, the best fit trajectory is extrapolated back to the storage region or forwards to the calorimeter in the extrapolation stage. A fourth refinement stage is planned but not yet implemented, which would add or remove hits in the finding stage based on the results of the fitting and extrapolation stages. This refinement is expected to improve statistics but not considerably improve performance.

As a brief aside, every stage of the track reconstruction is performed in the eventprocessing framework known as art 85. The art framework is a collection of modularized stages in a $\mathrm{C}++$ framework useful for reading, reconstructing, filtering, an-

alyzing, and writing data, among other things. Most Fermilab experiments now use art, including E989. 


\subsection{Track-finding}

The track-finding stage consists of pattern recognition routines which group individual hits into separate sets corresponding to individual tracks. The general implementation of these pattern recognition routines is relatively straightforward [86, 87]. Hits across all modules are grouped in time windows called time islands, with an average width of $40 \mathrm{~ns}$ and a max width of $100 \mathrm{n} \rrbracket$. Within those time islands hits are then grouped into clusters. Clusters consist of one or two hits for each $\mathrm{U}$ or $\mathrm{V}$ view per module. As a reminder, the $\mathrm{U}$ and $\mathrm{V}$ views of a module consist of the two $\mathrm{U}$ or $\mathrm{V}$ layers in that module. Hits are only clustered if they lie close in time and space to one another. The spatial constraint is defined as the difference in hit straw numbers, from 0 to 31 for the 32 straws per layer, which by default is limited to $\leq 4$. Neighboring hit clusters in the same module are then grouped to form seeds, one per module. Finally, seeds which are close in time and space are grouped together module by module from one end of the tracker to the other. The final data products are called track candidates. The entire track candidate formation process occurs for all hits in a time island to find as many real tracks as possible. See Figure $4 \cdot 1$.

After a track candidate has been formed, a number of checks are made before passing it on to the fitting stage. If hits, clusters, or seeds are shared among multiple track candidates, the candidates are dropped. Likewise, a track candidate is dropped if it is made from seeds taken from only one type of view, or if the track candidate has fewer than six hits. There are also various small geometry and timing algorithms to improve the track candidates, such as removing hits from secondaries [88]. The $t_{0}$ time for the track candidate is calculated as the mean time of all hits, with some fixed offset. The track candidate is supplied with an original momentum and position at the start of the track by fitting a circle to the hits in the horizontal plane. The final

\footnotetext{
${ }^{1}$ This time scale is set by the characteristic drift time.
} 


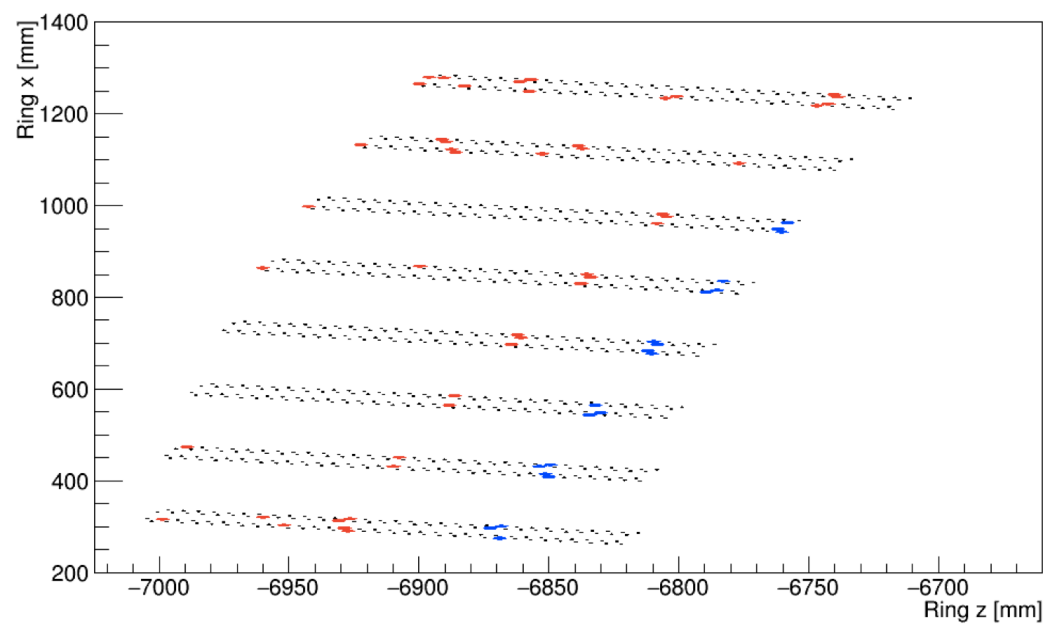

Figure 4.1: Hits in a tracker station in a single time island. The black dots indicate the position of the straw wires, while the blue and red points indicate hits. In blue is the first formed track candidate in the island, formed from separate seeds in different modules. The trackfinding algorithms will move onto the remaining hits in the time island to attempt to form other track candidates, one of which is easily observable by eye.

track candidates are then passed on to the fitting stage.

\subsection{Track-fitting}

The fitting stage takes the track candidates from the finding stage, and outputs a best fit trajectory to those candidates. This includes optimal state vectors and error matrices for the track at each measurement plane and at a fictitious starting plane at the entrance to the straw tracking detector. The track-fitting routines can roughly be split into two parts, error propagation and the actual fitting and improvement of the track. The implementation of these parts go hand in hand, and will be described in turn. Details of the track-fitting code itself is described in Reference [89]. 


\subsubsection{Error propagation and coordinate systems}

The process of error propagation involves taking track parameters and error matrices (which describe the uncertainties in those track parameters) and transporting them along discrete steps from one point to another, accounting for changes due to any magnetic fields or material along the step paths. There is a set of error propagation routines originally written in Fortran by the EMC collaboration, called "Geometry and Error Propagation" or Geane [90]. Geane calculates the evolution of the error matrix by propagating particles along their average trajectories neglecting the effects of discrete processes, using a helix equation along small enough steps where the change in the magnetic field is small. These routines were used in the E821 experiment as well as the PANDA and FINUDA experiments with some success 91. The Geane routines were at one point converted to $\mathrm{C}++$ and added to Geant. The strength of using Geane within a Geant simulation lies in its direct access to the Geant geometry and field. This is crucially important for the E989 track-fitting because the trackers live in a region of high field non-uniformity. Figure $4 \cdot 2$ shows the uniformity of the radial and vertical fields in the vicinity of the tracker as calculated in Opera2D and included in the E989 Geant4 simulation. The radial field in the tracker region rises from $0 \mathrm{~T}$ at the outer end to roughly $0.3 \mathrm{~T}$ at the inner top and bottom ends; the vertical field drops from the storage dipole field of $1.451 \mathrm{~T}$ at the outer end by approximately $50 \%$ at the inner end. These large field gradients over the tracking measurement space must be handled appropriately, which Geane does effectively.

Predicted average track parameters in Geane are a function of path length,

$$
\widetilde{p}_{l}=F_{l, l_{0}}\left(p_{0}\right),
$$

where $p_{0}$ are track parameters a distance $l_{0}$ along some path, and $\widetilde{p}_{l}$ are the updated average ones at a distance $l$. The path length from $l_{0}$ to $l$ can be defined or limited 


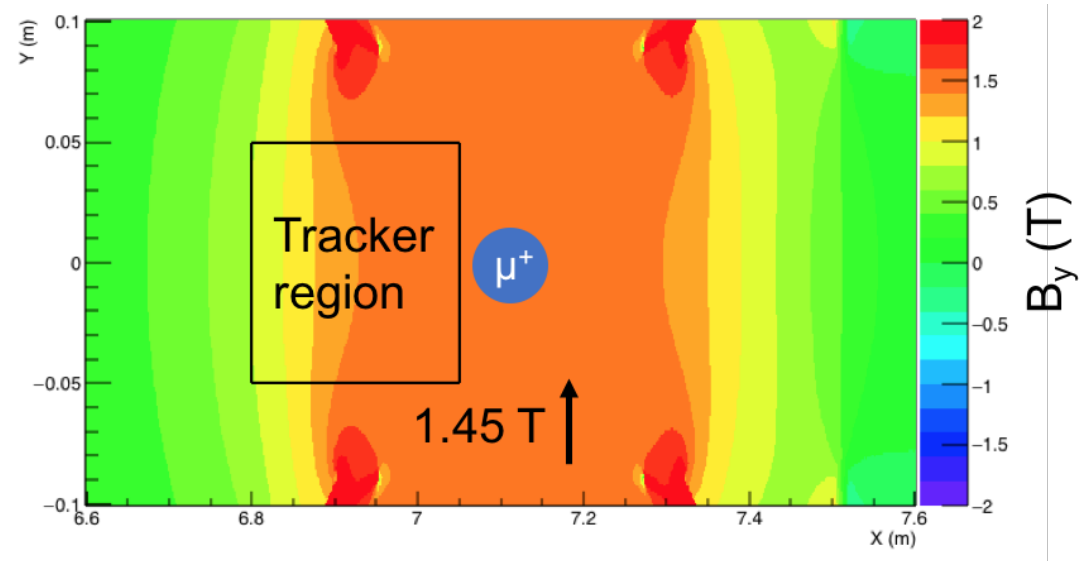

(a) Vertical magnetic field

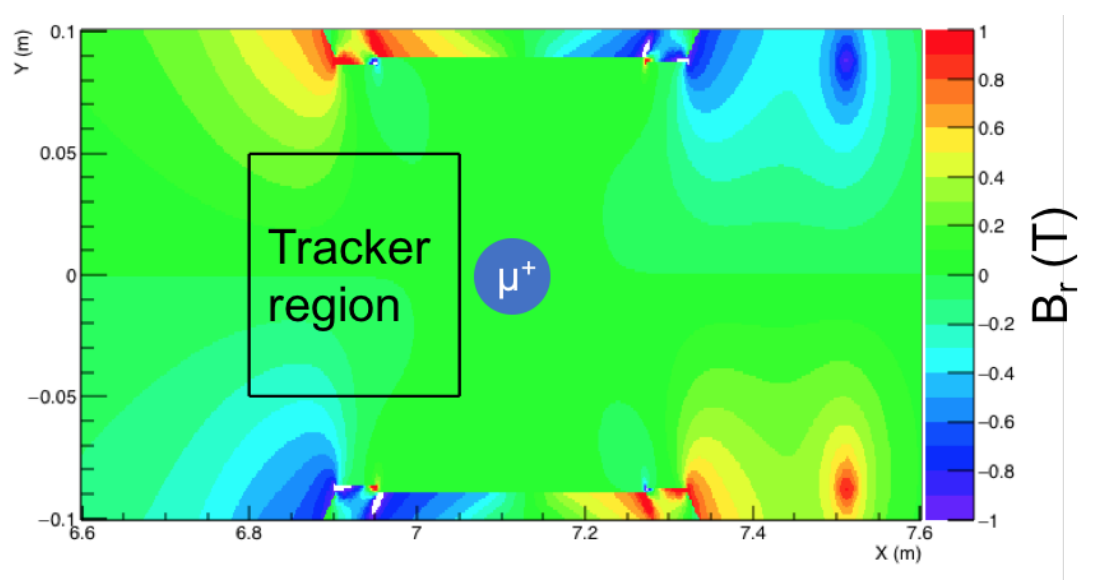

(b) Radial magnetic field

Figure $4 \cdot 2$ : Shown are the vertical (top) and radial (bottom) magnetic fields of the storage ring magnet in and around the storage region as calculated in Opera 2D. The horizontal and vertical axes are the radial and vertical coordinates of the ring respectively. The center of the storage region lies at $7.112 \mathrm{~m}$ along the horizontal axis. The contours represent the strengths of the vertical and radial magnetic fields. The black box shows the rough location of the tracker with respect to the ring. It can be seen that there is a large field non-uniformity within the tracker space. 
how one wishes, and typically corresponds to a single step in the Geant4 simulation. In the Geane routines the track parameters $p$ are $5 \times 1$ vectors defined in either the "free" (curvilinear) coordinate system

$$
\frac{1}{p}, \lambda, \phi, y_{\perp}, z_{\perp},
$$

or the "surface" (detector) coordinate system

$$
\frac{1}{p}, \frac{p_{v}}{p_{u}}, \frac{p_{w}}{p_{u}}, v, w
$$

In the free system, the $\lambda$ and $\phi$ parameters are the dip $(\pi / 2-\theta)$ and azimuthal angles respectively, while the $y_{\perp}$ and $z_{\perp}$ displacement parameters lie in the $\mathrm{XY}$ or XZ global Geant4 planes and orthogonal to $x_{\perp}$, where the $x_{\perp}$ coordinate lies along the momentum vector of the particle. See Figure 4.3. In the surface system, the UVW coordinates are defined with any two orthogonal vectors $\mathrm{V}$ and $\mathrm{W}$, where $\mathrm{V}$ and $\mathrm{W}$ typically lie in the surface of a detector ${ }^{2}$. The surface system is most usefully defined in the tracker reference frame, where the modules are staggered in a local $\mathrm{Z}(\mathrm{U})$ coordinate, the local $\mathrm{X}(\mathrm{V})$ coordinate increases with straw number, and the local $\mathrm{Y}(\mathrm{W})$ coordinate is vertical. See Figure $4 \cdot 4$. The surface system is then defined as

$$
\frac{1}{p}, \frac{p_{x}}{p_{z}}, \frac{p_{y}}{p_{z}}, x, y
$$

In both free and surface systems, the track is represented by one momentum parameter, two directional parameters, and two position parameters. Needing six independent parameters to describe a particle in space and momentum (three momentum and three position parameters), one parameter is selected as an independent

\footnotetext{
${ }^{2}$ For clarification, the UVW surface system has nothing to do with the UV orientations of the straws at this time.
} 

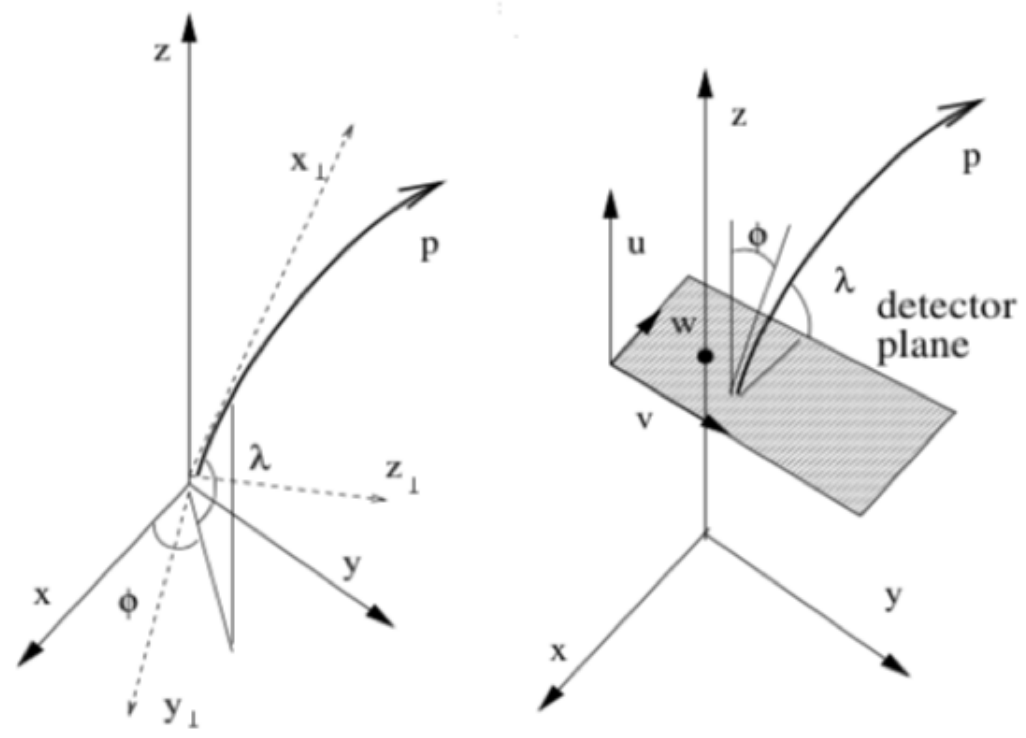

Figure 4.3: Free (left) and surface (right) tracking coordinate systems 91].

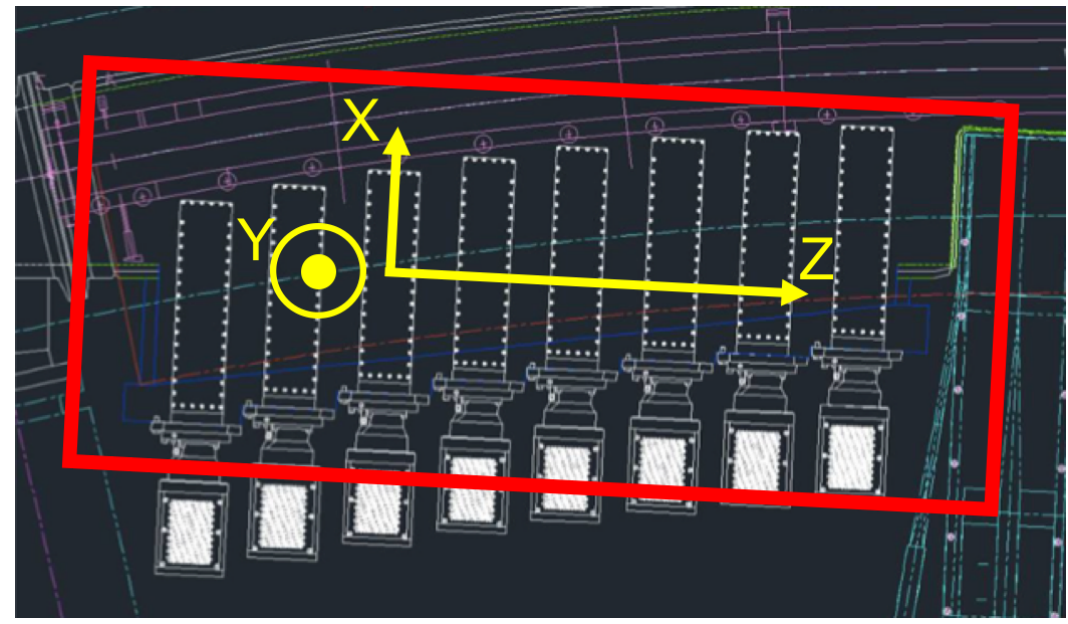

Figure 4.4: Model view of a tracker station in relation to the storage ring. Tracker modules are outlined in white. Around the tracker measurement area is defined a coordinate system called the tracker reference frame. In this frame, the $\mathrm{X}$ coordinate is directed nearly radially outward along the straws, the $\mathrm{Y}$ coordinate is directed vertically up, and the $\mathrm{Z}$ coordinate is directed along the direction that the tracker modules are staggered. 
variable. For Geane, this is taken either as a known path length in the free system, or a known $\mathrm{U}$ coordinate in the surface system (or known $\mathrm{Z}$ coordinate in our tracker reference frame). In our tracker reference frame, the 32 straw layers corresponding to a tracking station are defined at known local $\mathrm{Z}$ coordinates. The path lengths for steps in Geane can be set equal to the distance for a track to travel between between detector planes. Therefore the track parameters' dependence on the path length can instead by replaced by a dependence on plane number. The number of degrees of freedom per track is the number of measurement planes it hits, $N$, minus 5 for the number of track parameters.

The $5 \times 5$ error matrix on a plane calculated in Geane describing the expected distribution in true parameters about the average ones is defined as

$$
\sigma_{N}^{i j}=\left\langle p_{N}^{i} p_{N}^{j}\right\rangle-\left\langle p_{N}^{i}\right\rangle \cdot\left\langle p_{N}^{j}\right\rangle
$$

where $\mathrm{i}$ and $\mathrm{j}$ are track parameter indices, and $p_{N}$ are 'true' track parameters on plane $N$. This error matrix will include effects from multiple scattering, delta ray production, ionization, and bremsstrahlung 90, 91, 92. These matrices are evolved from plane to plane by what are called transport matrices, where the $5 \times 5$ transport matrix elements between two planes are defined as

$$
T_{N, N-1}^{i, j}=\frac{\partial \widetilde{p}_{N}^{i}}{\partial p_{N-1}^{j}}
$$

The transport matrix $T$ is a Jacobian which describes how the infinitesimal changes in parameter $i$ on plane $N$ (or path length) are produced by changes in parameter $j$ on some previous plane $N-1$ :

$$
\delta \widetilde{p}_{N}=T_{N, N-1} \delta p_{N-1}
$$

Note that the transport matrix does not propagate the track parameters themselves 
as does an equation of motion. The error matrix is propagated forward from one plane to another by

$$
\sigma_{N}=T_{N, N-1} \sigma_{N-1} T_{N, N-1}^{T}+\sigma_{\text {material }}
$$

where $\sigma_{\text {material }}$ is the added error due to material effects between the planes. See Figure 4.5. The calculation of the transport matrices themselves is done within the Geane routines in the free system on a step by step basis, where the derivation of the transport matrix elements is given in Reference [93]. It should be pointed out that the transport matrix between any two planes (or number of steps) is the multiple of all intermediate transport matrices,

$$
T_{N, N-2}=T_{N, N-1} T_{N-1, N-2},
$$

regardless of what reference system the matrices are defined in (as long as they are all consistent). Geane can convert the transport matrices between the free system and the surface system using further Jacobians, also derived in Reference [93]. When converting a transport matrix from one reference system to another,

$$
T_{N, N-1}^{s}=A_{N} T_{N, N-1}^{f} A_{N-1}^{-1},
$$

where the $s$ and $f$ superscripts stand for the surface and free reference systems respectively, and $A$ is the Jacobian between reference frames which is defined at a specific point or plane $\left(A_{N} \neq A_{N-1}\right)$. The error matrices are converted from one reference frame to another in the usual way,

$$
\sigma_{N}^{s}=A_{N} \sigma_{N}^{f} A_{N}^{T} .
$$

Finally, while the tracker reference frame is nominally defined in the local XYZ 


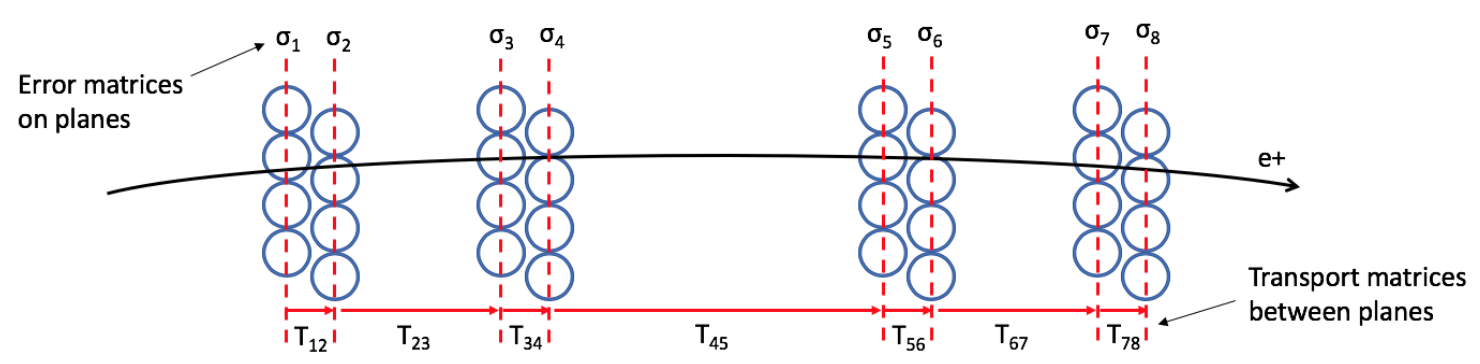

Figure 4.5: Transport and error matrices for straw tracker planes. Transport matrices are defined between straw planes, and error matrices are defined on the planes.

coordinates as described previously, the straws themselves do not measure coordinates in that frame directly. As described in Section 3.3, the straws measure drift circles in planes perpendicular to the straws themselves. The measurements from $\mathrm{U}$ and $\mathrm{V}$ straws therefore lie on the $\mathrm{U}$ and $\mathrm{V}$ measurement axes shown in Figure 4.6, where the measurement of the drift circle radius is instead taken as a $\mathrm{U}$ or $\mathrm{V}$ coordinate to the left or right of the straw wire. To first order, the $\mathrm{U}$ or V coordinate is the DCA of the hit, which can be corrected with the angle of the track to get a better estimate, as shown in Appendix A.1. It's important to note that out of the five track parameters, each straw only measures a single $\mathrm{U}$ or $\mathrm{V}$ position. The new coordinate system is defined as

$$
\frac{1}{p}, \frac{p_{u}}{p_{z}}, \frac{p_{v}}{p_{z}}, u, v,
$$

where this $Z$ variable is the tracker reference frame $Z$, and the $\mathrm{U}$ and $\mathrm{V}$ coordinates here are non-orthogonal and different from those in Equation 4.3. The transformation between the XYZ and UVZ systems is given by

$$
p^{U V}=J_{5} p^{X Y}
$$




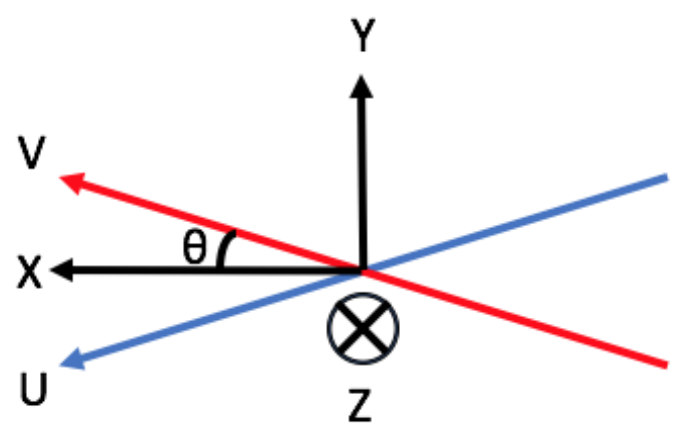

Figure 4.6: The straw tracker measurement reference system. The XYZ system here is the straw tracker reference frame. $\theta$ is the same angle as the stereo angle of the straws, at $7.5^{\circ}$. U straws measure along the $\mathrm{U}$ axis and $\mathrm{V}$ straws measure along the $\mathrm{V}$ axis.

where $J_{5}$ is a $5 \times 5$ matrix defined by

$$
J_{5}=\left(\begin{array}{ccc}
1 & 0 & 0 \\
0 & J_{2} & 0 \\
0 & 0 & J_{2}
\end{array}\right)
$$

and $J_{2}$ is a $2 \times 2$ matrix given by

$$
\left(\begin{array}{l}
u \\
v
\end{array}\right)=J_{2}\left(\begin{array}{l}
x \\
y
\end{array}\right)=\left(\begin{array}{cc}
\cos \theta & -\sin \theta \\
\cos \theta & \sin \theta
\end{array}\right)\left(\begin{array}{l}
x \\
y
\end{array}\right)
$$

$J_{2}$ is readily determined by inspection of Figure $4 \cdot 6$. In order to transform the transport or error matrices from the tracker reference frame to the tracker measurement frame, the same relations as in Equations 4.10 and 4.11 apply,

$$
\begin{aligned}
T_{N, N-1}^{U V} & =J_{5} T_{N, N-1}^{X Y} J_{5}^{-1} \\
\sigma_{N}^{U V} & =J_{5} \sigma_{N}^{X Y} J_{5}^{T}
\end{aligned}
$$

where the superscripts of XY or UV identify which coordinate system the objects belong to. 


\subsection{2 $\chi^{2}$ minimization}

The method for fitting and improving the track is a global $\chi^{2}$ minimization algorithm

that uses the transport and error matrices as described previously [90, 94. This straightforward global fitting algorithm works because of the minimal amount of material contained within the tracker and the resulting small correlations between planes. For denser detectors with more scattering and therefore greater correlations, other fitting algorithms such as a Kalman filter should be used 91. While the following derivations and minimization correspond to measurements on planes in the tracker measurement frame described by Equation 4.12, it should be noted that the results apply to any reference frame. A derivation for a $\chi^{2}$ including no material correlations is presented, followed by one which includes material correlations.

The $\chi^{2}$ for a track is defined as the squared residuals between predicted and measured parameters on a measurement plane, divided by their errors, summed over all hit planes:

$$
\chi^{2}=\sum_{i=1}^{N}\left[\left(\widetilde{p}_{i}\left(p_{s}\right)-x_{i}\right)^{T}\left(\sigma_{i}^{-1}\right)\left(\widetilde{p}_{i}\left(p_{s}\right)-x_{i}\right)\right]
$$

$x_{i}$ are vectors of the measured track parameters on plane $i, \widetilde{p}_{i}$ are vectors of the average predicted track parameters which stem from the starting parameters $p_{s}$, and $\sigma_{i}$ are the $5 \times 5$ error matrices on the planes. To first order, the error matrices consist only of the measurement errors on the $\mathrm{U}$ and $\mathrm{V}$ parameters and exclude the effects of random material processes. These errors are located in the $\mathrm{U}$ and $\mathrm{V}$ diagonal elements $(3,3)$ and $(4,4)$ respectively, with corresponding resolutions of approximately $150 \mu \mathrm{m}$ as described in Section 3.3. At second order, the material error matrices as calculated by Geane are added to the measurement errors. Because the measured parameters consist of solely $\mathrm{U}$ or $\mathrm{V}$ measurements, the $x_{i}$ vectors are $5 \times 1$ objects where only the 
(3) or (4) elements have any meaning respectively ${ }^{3}$. The errors on the non-measured parameters in the diagonals of the error matrix are taken as infinite. When the error matrix is inverted all corresponding rows and columns of the final matrix calculation on each plane reduce to zero and contribute nothing to the $\chi^{2}$.

By minimizing this $\chi^{2}$ with respect to the starting parameters $p_{s}$, and evaluating it at the target best starting guesses $p_{0}^{\prime}$, which are the parameters of interest, the track can be fit:

$$
\begin{aligned}
\left.\frac{\partial \chi^{2}}{\partial p_{s}}\right|_{p_{s}=p_{0}^{\prime}}=0 & =\sum_{i=1}^{N}\left[\left(\left.\frac{\partial \widetilde{p}_{i}\left(p_{s}\right)}{\partial p_{s}}\right|_{p_{s}=p_{0}^{\prime}}\right)^{T}\left(\sigma_{i}^{-1}\right)\left(\widetilde{p}_{i}\left(p_{0}^{\prime}\right)-x_{i}\right)\right. \\
& +\left(\widetilde{p}_{i}\left(p_{0}^{\prime}\right)-x_{i}\right)^{T}\left(\left.\frac{\partial\left(\sigma_{i}^{-1}\right)}{\partial p_{s}}\right|_{p=p_{0}^{\prime}}\right)\left(\widetilde{p}_{i}\left(p_{0}^{\prime}\right)-x_{i}\right) \\
& \left.+\left(\widetilde{p}_{i}\left(p_{0}^{\prime}\right)-x_{i}\right)^{T}\left(\sigma_{i}^{-1}\right)\left(\left.\frac{\partial \widetilde{p}_{i}\left(p_{s}\right)}{\partial p_{s}}\right|_{p_{s}=p_{0}^{\prime}}\right)\right]
\end{aligned}
$$

The middle term is small and can be neglected, assuming that that the error matrix doesn't change much with respect to the choice of starting parameters. This is true as the part of the error matrix that depends on the starting parameters is already small due to the small amount of material in the tracker. In tandem, the error matrix doesn't change much from one fitting iteration to the next as long as the path length through the material remains about the same. The first and third terms are identical in value, and so must therefore both separately be equal to zero. Equation 4.19 is therefore reduced to

$$
0=\sum_{i=1}^{N} T_{i 0}^{T} \sigma_{i}^{-1}\left(\widetilde{p}_{i}\left(p_{0}^{\prime}\right)-x_{i}\right)
$$

where $T_{i 0}$ is the transport matrix between the point at which the starting parameters

\footnotetext{
${ }^{3} \mathrm{~A}$ straw tracker module as a whole can be approximated as measuring in $2 \mathrm{D}$ space, but this leads to correlations between measured parameters which must be taken into account, as compared to the natural tracker measurement frame in $1 \mathrm{D}$ space of $\mathrm{U}$ or $\mathrm{V}$ for which there are no measurement correlations 95 .
} 
are defined and plane $i$, given by Equation 4.6 .

$$
T_{i 0}=\left.\frac{\partial \widetilde{p}_{i}\left(p_{s}\right)}{\partial p_{s}}\right|_{p_{s}=p_{0}^{\prime}}
$$

In minimizing the $\chi^{2}$, the desire is to update some original set of starting track parameters $p_{0}$ to the new best ones $p_{0}^{\prime}$. This difference, $\Delta p_{0}$, can be determined by substituting the following into Equation 4.20 .

$$
\widetilde{p}_{i}\left(p_{0}^{\prime}\right)=\widetilde{p}_{i}\left(p_{0}\right)+T_{i 0} \Delta p_{0}
$$

which follows from Equation 4.7. After simplifying one arrives at

$$
\Delta p_{0}=\sigma_{p_{0}} \sum_{i=1}^{N} T_{i 0}^{T}\left(\sigma_{i}^{-1}\right)\left(x_{i}-\widetilde{p}_{i}\left(p_{0}\right)\right)
$$

where

$$
\sigma_{p_{0}}=\left[\sum_{i=1}^{N} T_{i 0}^{T}\left(\sigma_{i}^{-1}\right) T_{i 0}\right]^{-1}
$$

$\sigma_{p_{0}}$ is a $5 \times 5$ covariance matrix of the starting fit parameters, where the diagonals describe the fit errors in the 5 track parameters at that point.

To summarize, an initial set of starting parameters $p_{0}$ are propagated forwards in Geane to produce predicted track parameters, transport matrices, and error matrices. These objects, along with the measured parameters, are used in the $\chi^{2}$ minimization algorithm to provide a $\chi^{2}$ describing the track goodness-of-fit corresponding to those original starting parameters, an improvement on the starting parameters $\Delta p_{0}$, and the errors on those parameters $\sigma_{p_{0}}$. One iteration of the track-fitting consists of these two steps. In order to determine the predicted parameters of the track corresponding to the improved starting parameters, the error propagation part of the procedure needs to be repeated. The track-fitting is iterated until the $\chi^{2}$ no longer improves, at which 
point the track-fitting is said to have converged. Typically three or four iterations are enough to get a best fit track. As a reminder, the initial set of starting parameters is given by a circle fit to the hits as described at the end of Section 4.1. The starting parameters for a track are defined on a virtual 0 plane parallel to the measurement planes, where the 0 plane is placed on a track by track basis at a point $1 \mathrm{~cm}$ in front of the first straw tracker module that was hit. Note that the track-fitting procedure is remarkably robust with respect to the initial starting parameters. Of course if the initial starting parameters are too poor, then the fit will not converge.

\subsubsection{Fits to idealized tracks in vacuum}

The tracking algorithm was built and tested in the full E989 Geant4 simulation in art. Hits were generated from positrons produced by muon decay in the storage region. In the initial tests of the track-fitting, material was turned off and the measured hits were taken as the truth hits with some known Gaussian smearing. (The truth hits are accessible within the Geant4 simulation by "dummy plane" detectors which record hits at the straw measurement planes.) Plots showing the goodness-of-fit for the fitted tracks are shown in Figure 4.7. Beyond the goodness-of-fit, the other measures of track-fitting performance are the truth pulls of the fit parameters. The truth pulls are defined as the residual between the fitted parameter and the truth parameter, divided by the fit error on that parameter:

$$
\frac{\Delta p_{0}^{i}}{\sqrt{\sigma_{p_{0}}^{i i}}}=\frac{p_{0, \mathrm{fit}}^{i}-p_{0, \text { true }}^{i}}{\sqrt{\sigma_{p_{0}}^{i i}}}
$$

Since the $\chi^{2}$ minimization returns fit parameters and errors on the starting plane, this is where the truth pulls are defined. Plots of the truth pulls for the five track parameters are shown in Figure 4.8, where each pull is a unit Gaussian, as they should be for idealized results. 

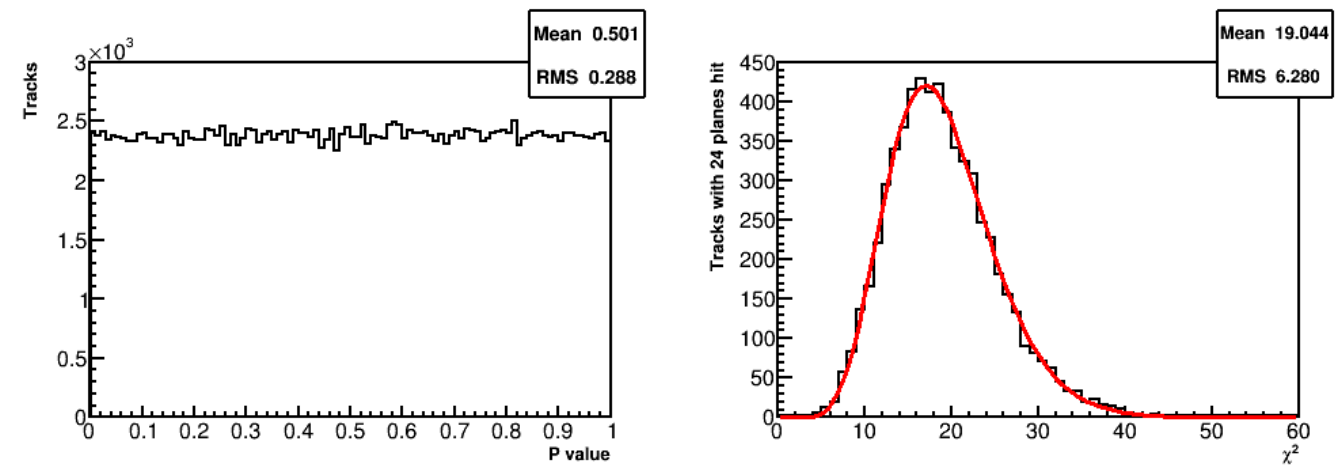

(a) p-value distribution for all tracks.

(b) $\chi^{2}$ distribution for tracks which hit 24 planes.

Figure 4·7: Goodness-of-fit distributions for fitted tracks in vacuum, with Gaussian measurement errors and no material effects. The pvalue distribution is flat, and the $\chi^{2}$ distribution matches a normalized $\chi^{2}$ probability distribution function for 19 degrees of freedom which is overlaid in red. ( $\chi^{2}$ distributions for tracks which hit other numbers of planes are very similar.)

\subsubsection{Material correlations}

While the trackers consist of relatively little material, for improved tracking results it is desirable to include the full effects of material. As described in Section 4.2.1, random processes such as multiple scattering and ionization contribute to the error matrix in Equation 4.5. The random scattering of a particle trajectory at one plane means that there is an extra correlated error in all further planes, see Figure $4 \cdot 9$, Equation 4.18 does not take into account these material correlations between measurement planes when fitting the track. While it provides a decent approximation of the best fit track in the low material tracker, the $\chi^{2}$ distribution is noticeably wrong. To calculate a better estimate of the trajectory, a more general version of the $\chi^{2}$ equation is used:

$$
\chi^{2}=(\vec{p}-\vec{x})^{T}\left(\sigma^{-1}\right)(\vec{p}-\vec{x})
$$




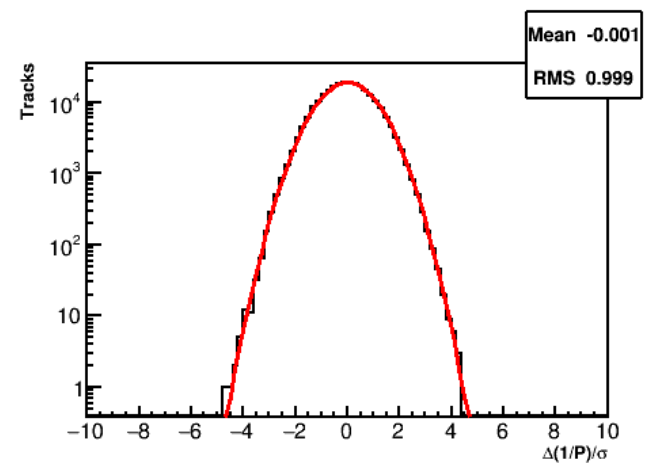

(a) $1 / P$

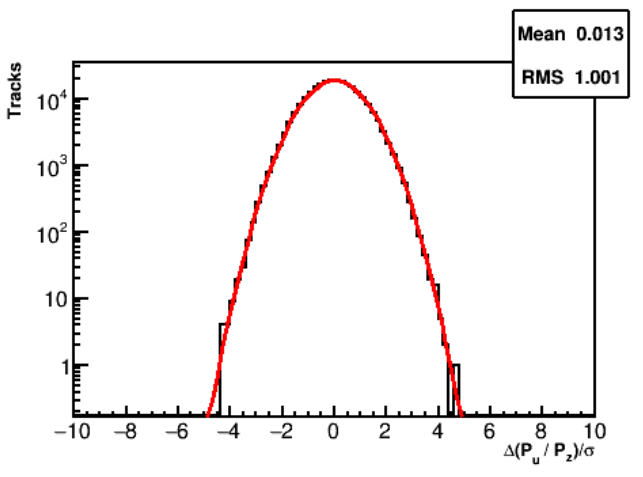

(b) $P_{u} / P_{z}$

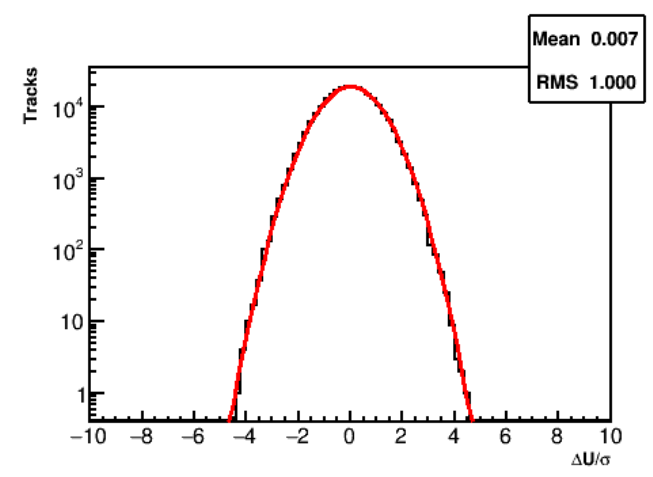

(d) $U$

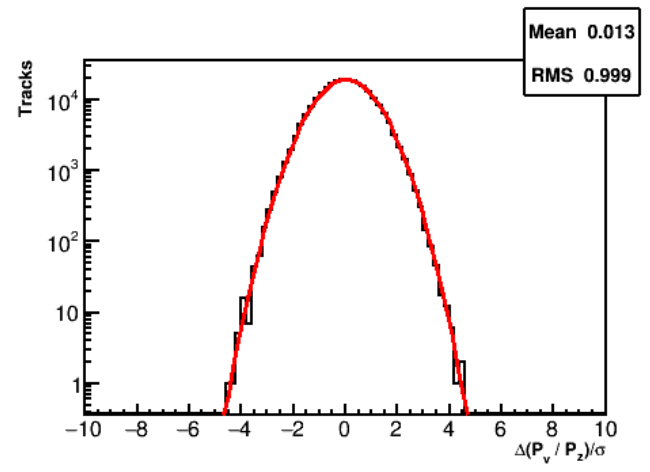

(c) $P_{v} / P_{z}$

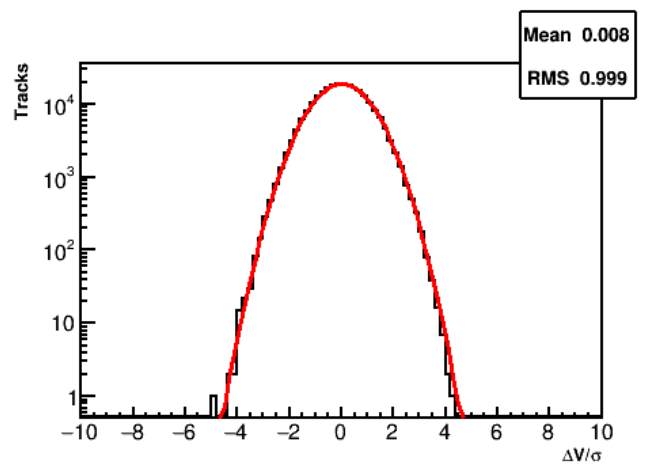

(e) $V$

Figure 4.8: Truth pulls for the five fitted track parameters at the starting plane of the track, for tracks in vacuum with Gaussian measurement smearing and no material effects. The plots are shown on a log scale, and each are fit to a Gaussian in red. Each fit is consistent to a unit Gaussian with a mean of zero and an RMS of one, showing that the track-fitting is working properly. 


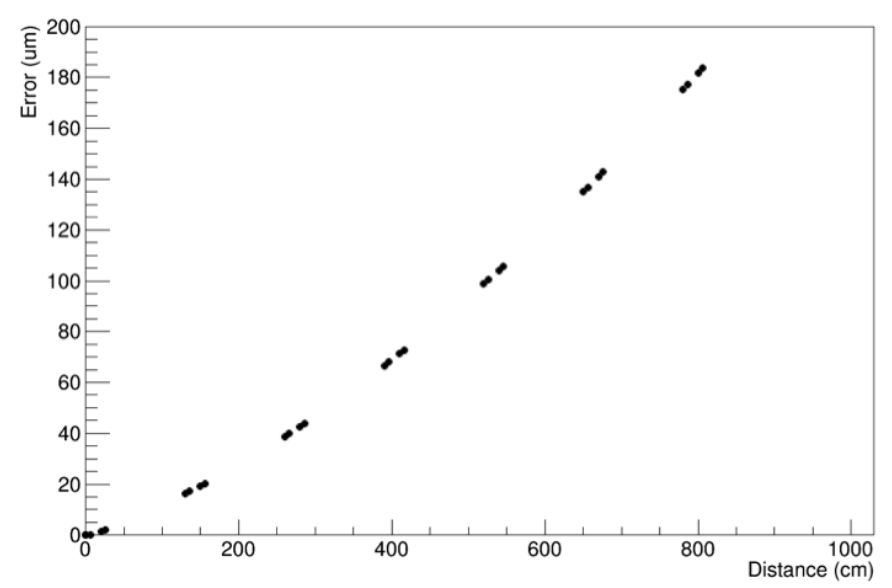

Figure 4.9: RMS error between the true track position and the average track position as a function of distance through the tracker. This error increases as a particle passes through more and more material. Each black point indicates the location of a straw measurement layer. If a track goes through the whole detector, on average the true position has an extra uncertainty of nearly $200 \mu \mathrm{m}$ compared to the average one.

Here $\vec{x}$ and $\vec{p}$ are a $5 N \times 1$ vectors of the measured and predicted track parameters respectively, where $N$ is the number of planes hit, and these objects are the combined vectors of the $5 \times 1$ counterparts. Similarly, $\sigma$ is a $5 N \times 5 N$ matrix, where the $5 \times 5$ diagonal block matrices are the individual plane error matrices described before, calculated between plane 0 and $N$. Calculating the $\chi^{2}$ is now recast from a sum over measurement planes into a single large linear algebra equation. At this point both calculations of the $\chi^{2}$ are equivalent.

The new format however allows for the material correlations between planes to be included, where these correlations are added as $5 \times 5$ matrices in the off-diagonal blocks of the new large error matrix. The upper diagonals are given by ${ }^{4}$

$$
\sigma_{M N}=T_{M N} \sigma_{N}
$$

where $\sigma_{M N}$ is the material correlation matrix between plane $M$ and plane $N, T_{M N}$ is

\footnotetext{
${ }^{4}$ The lower diagonals are just the transpose.
} 
the transport matrix between the two planes, and $\sigma_{N}$ is the ordinary material error matrix as calculated from the 0 starting plane. See Appendix A.2 for the derivation of Equation 4.27. The $\chi^{2}$ is minimized in the same as in the previous section such that the improvement to the starting track parameters $\Delta p_{0}$ remains a $5 \times 1$ vector and is given by

$$
\begin{aligned}
\Delta p_{0} & =\sigma_{p_{0}} \tau^{T} \sigma^{-1}(\vec{x}-\vec{p}) \\
\sigma_{p_{0}} & =\left[\tau^{T} \sigma^{-1} \tau\right]^{-1}
\end{aligned}
$$

where the joint transport matrix $\tau$ is a $5 N \times 5$ object, consisting of $N$ ordinary transport matrices tacked together.

Because $\sigma$ is such a large matrix, $5 N \times 5 N$ where $N$ ranges from 6 to 32 , the computational burden of inverting it for many tracks would be excessive. However, as described before, the infinite errors in the diagonals for non-measured parameters reduce the corresponding rows and columns to 0 after the inversion. This fact can be taken advantage of by removing said rows and columns that would contribute nothing to the $\chi^{2}$ anyway, and thus reducing the dimension from $5 N \times 5 N$ to $N \times N$. The corresponding rows and columns of the unmeasured parameters in the combined transport matrix $\tau$ and residual vector are also removed, resulting in an $N \times 5$ matrix for $\tau$ and an $N \times 1$ vector for the residuals. The covariance matrix $\sigma_{p_{0}}$ remains a $5 \times 5$ matrix. These reductions in the matrix dimensions improve the speed of the $\chi^{2}$ calculation dramatically, while leaving the final calculation unaffected ${ }^{5}$. All pieces of the $\chi^{2}$ calculation and minimization are done with a $\mathrm{C}++$ linear algebra library optimized for speed called Eigen3 [96]. As a result, the tracking time is dominated by the error propagation in Geant4, and not the linear algebra.

\footnotetext{
${ }^{5}$ Note that these element removals are done just before the final calculation of the $\chi^{2}$ and not at the beginning of the algebra, otherwise the plane material correlations are not properly included.
} 


\subsubsection{Fits to simulated tracks including material effects}

The inclusion of material correlations repairs the $\chi^{2}$ distributions as shown in Figure $4 \cdot 10$. The plots in this section show the results of the track-fitting in the full $g-2$ Geant4 simulation with material effects turned on. Truth measurements with $150 \mu \mathrm{m}$ Gaussian smearing are once again used as the measured hits, and a cut of greater than $3 \mathrm{MeV}$ on the true simulated energy loss removes the occasional kinked tracks which produce poor fits from hard energy loss physics processes. The comparison between the simulated and reconstructed energy loss for fitted tracks is shown in Figure $4 \cdot 11$. Truth pulls for the tracks are shown in Figure $4 \cdot 12$, It can be seen that there is a slight spread in results due to the material effects. This is to be expected given the non-Gaussian nature of the processes, and the vast majority of tracks still fit well. The number of iterations required to fit a track is shown in Figure $4 \cdot 13$. The number of planes a track hits and the corresponding momentum dependence is shown in Figure $4 \cdot 14$. The total momentum distribution and residuals to truth are shown in Figure $4 \cdot 15$. The momentum resolution of the track-fitting is approximately $2 \%$.

After the track-fitting has determined the best fit parameters in the UV space, the returned parameters can be transformed back into the tracker reference frame coordinates. Plots for the fitted vertical and horizontal momenta, positions, and corresponding residuals are shown in Figures $4 \cdot 16$ and $4 \cdot 17$. More extensive plots can be found in Reference 89. 

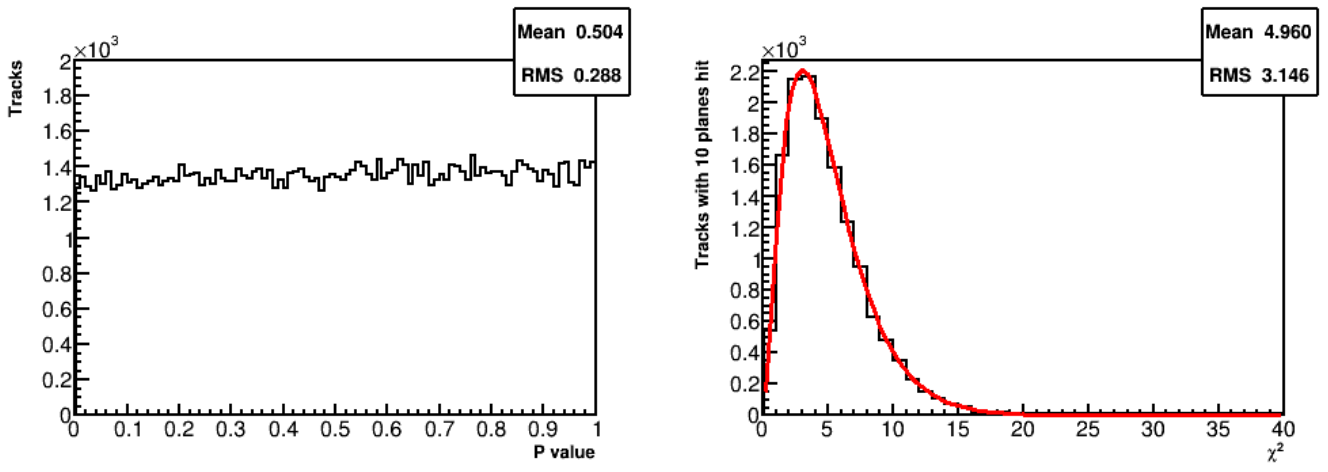

(a) p-value distribution for all tracks.

(b) $\chi^{2}$ distribution for tracks which hit 10 planes.

Figure 4.10: Goodness-of-fit distributions for fitted tracks in the full $g-2$ Geant 4 simulation with material effects and correlations included. The p-value distribution is flat, and the $\chi^{2}$ distribution matches a normalized $\chi^{2}$ probability distribution function for 5 degrees of freedom which is overlaid in red.

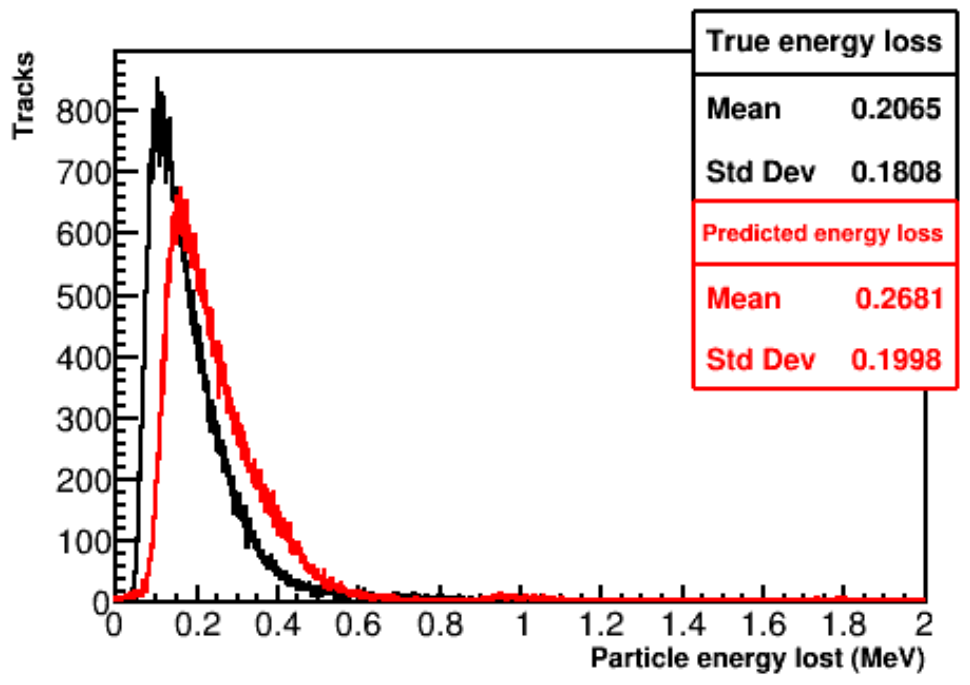

Figure 4.11: Simulated true energy loss (black) vs Geane predicted energy loss (red) for fitted tracks. The mismatch between the two is acceptable as the energy loss is in general very small compared to the total momentum of each track, $200 \mathrm{keV} \ll 2 \mathrm{GeV}$. Sources of energy loss include ionization and bremsstrahlung processes. The original Geane physics calculations were taking too much energy away due to bremsstrahlung processes in our low material tracker, so the energy loss calculations were modified slightly [89]. 


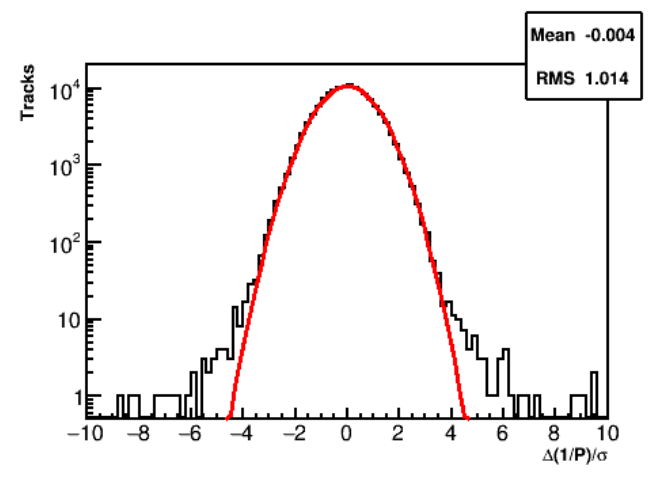

(a) $1 / P$

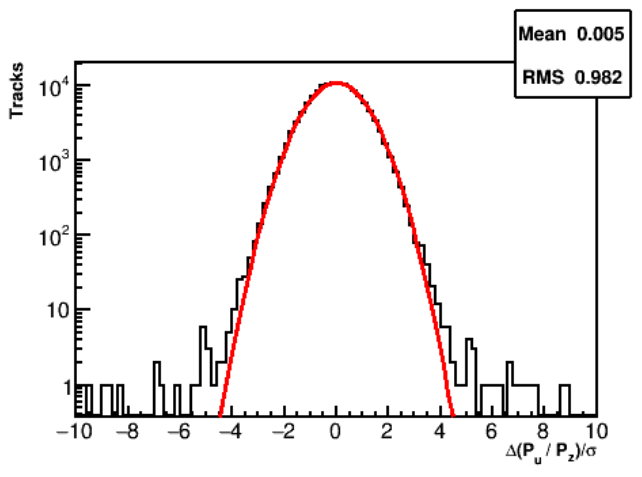

(b) $P_{u} / P_{z}$

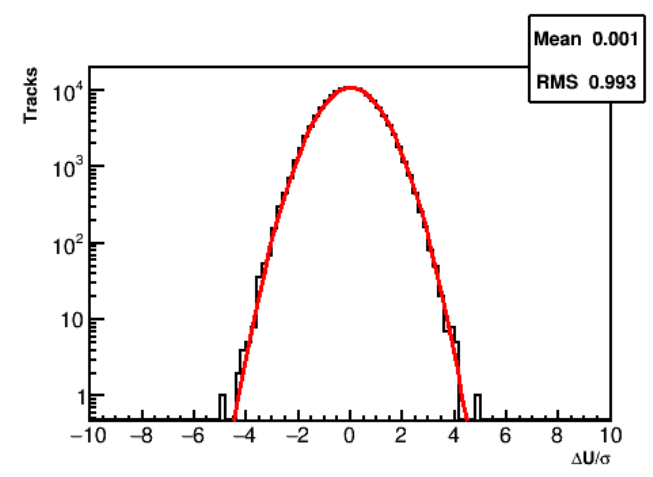

(d) $U$

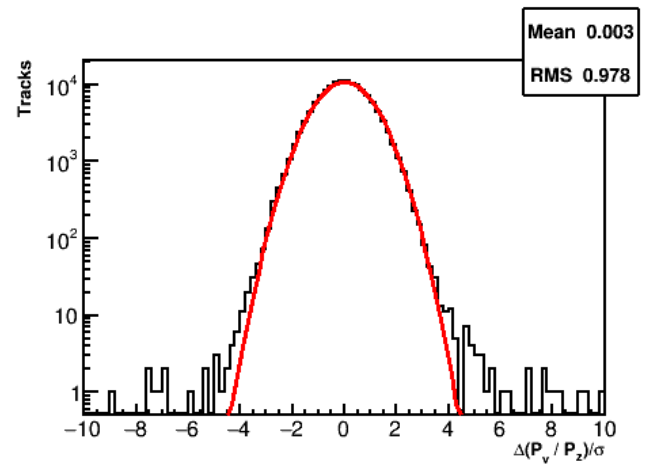

(c) $P_{v} / P_{z}$

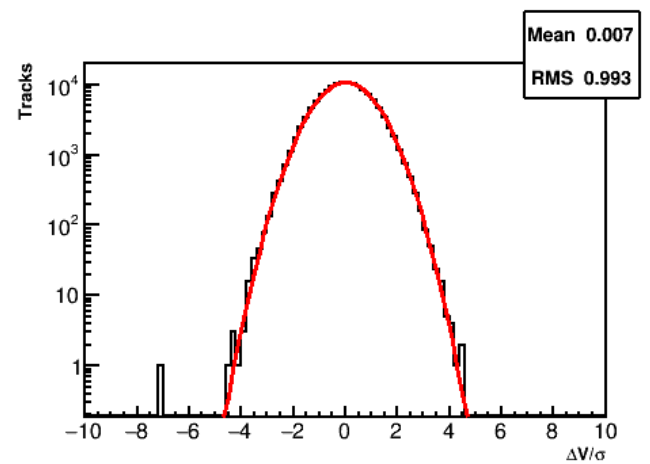

(e) $V$

Figure 4.12: Truth pulls for the five track parameters at the starting plane of the track for fitted tracks in the full $g-2$ Geant4 simulation with material effects included. The plots are shown on a log scale, and each is fit to a Gaussian in red. Each fit is close to a unit Gaussian with a mean of zero and an RMS of 1 , but there are tracks which lie outside the Gaussian due to material effects and the resulting imperfect fits. 


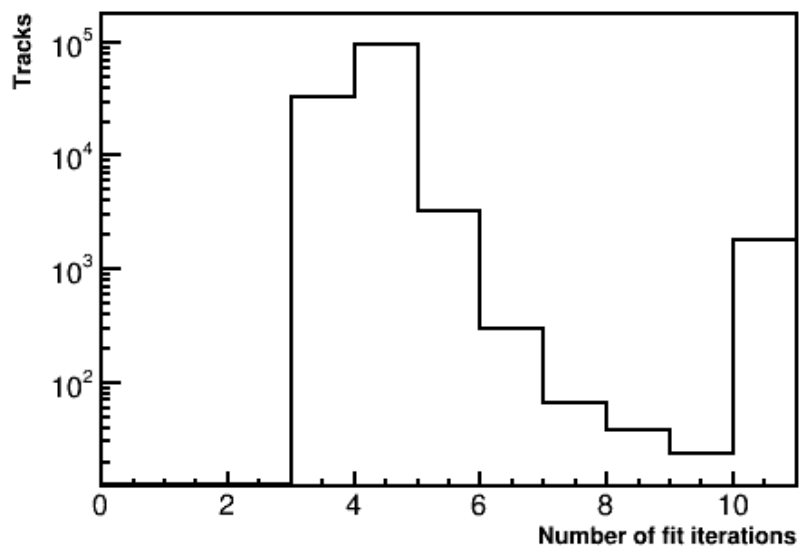

Figure 4.13: Number of iterations for the track-fitting to converge per track. The track-fitting does not take less than three iterations, and the number of iterations is capped at ten.

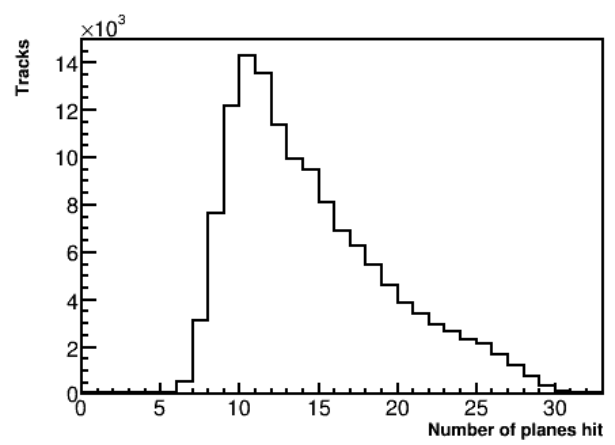

(a) The number of planes hit peaks at 10 , and falls off to the maximum number of planes at 32 .

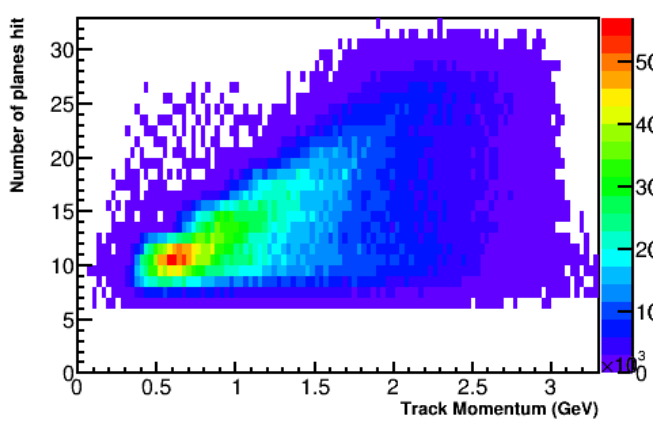

(b) The number of planes hit vs the momentum of the track. In general, tracks with higher momenta hit more planes.

Figure 4.14: Number of planes hit per track (left) and the momentum dependence (right). 


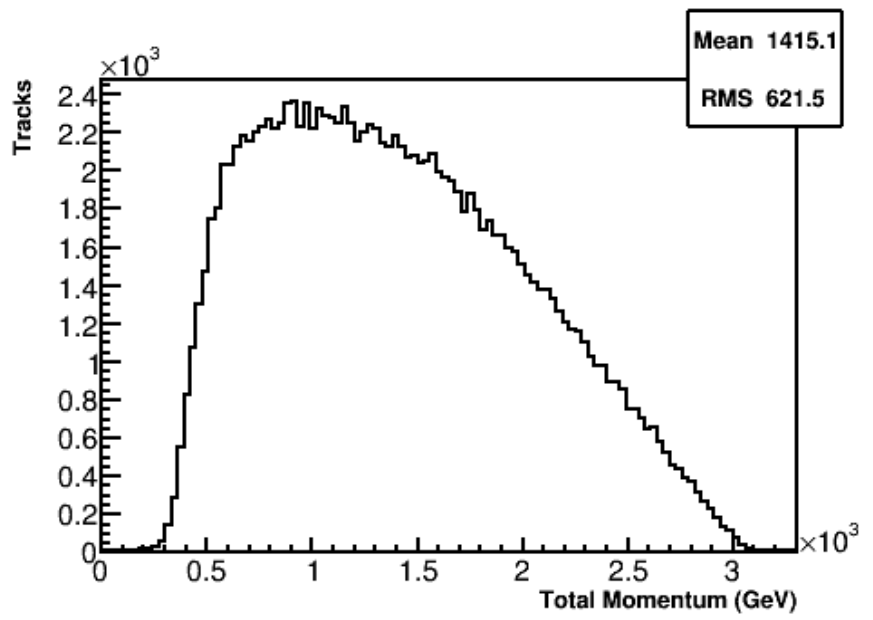

(a) The fitted momentum distribution, which has an endpoint of approximately $3.094 \mathrm{GeV}$.

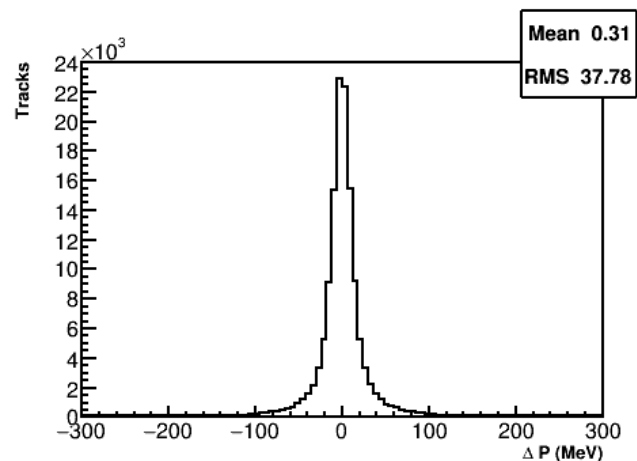

(b) The residual between the reconstructed and true total momentum. The RMS is approximately $40 \mathrm{MeV}$, though there are some tails which spread out from the distribution.

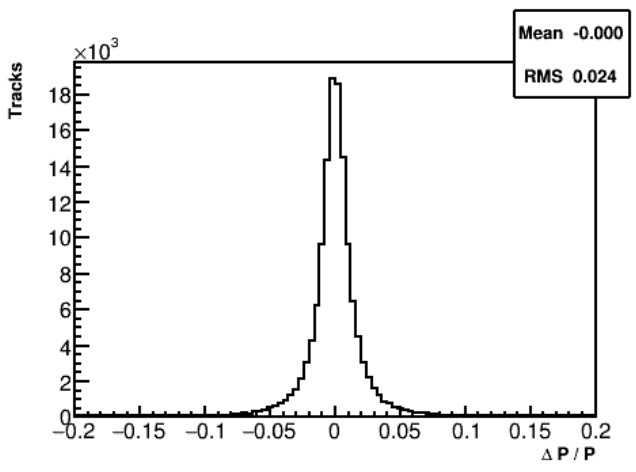

(c) The relative residual between the reconstructed and true total momentum. The RMS is approximately $2.4 \%$. This plot includes tracks of all momenta; in general the resolution of the total momentum is around $2 \%$ for all energies.

Figure 4.15: Fitted track momentum distribution and corresponding residuals to truth. 


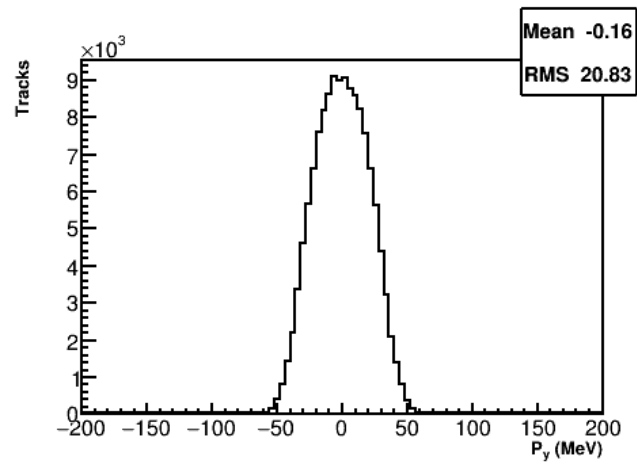

(a) Vertical momentum distribution. This distribution is bounded by the vertical angle acceptance of the tracker.

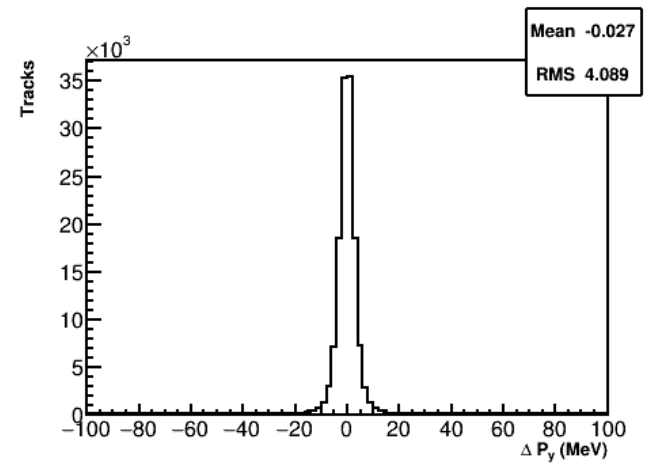

(c) The vertical momentum residual, which has a width of about $4 \mathrm{MeV}$.

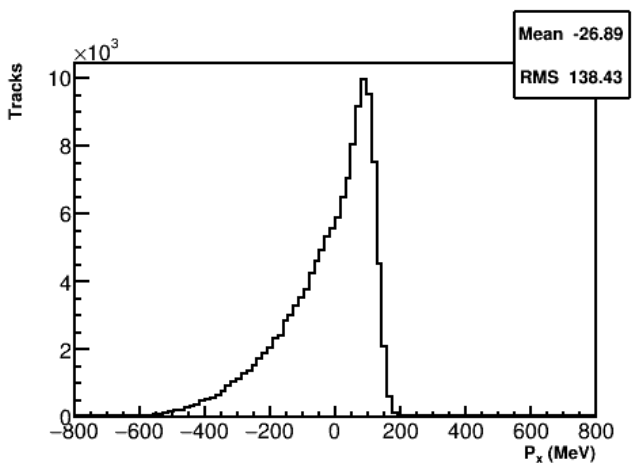

(b) Horizontal momentum distribution. The shape of the distribution is due to the acceptance of the tracker. It is perhaps more informative to look at the radial momentum distribution, which is shown in Figure $4 \cdot 20$.

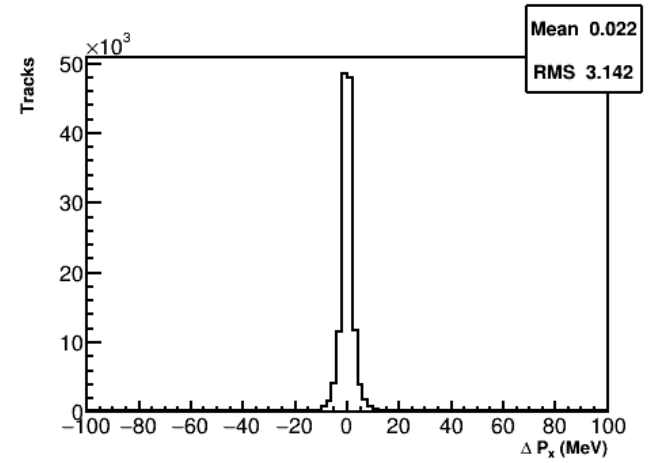

(d) The horizontal momentum residual, which has a width of about $3 \mathrm{MeV}$.

Figure 4.16: Fitted vertical and horizontal momentum components (top) for tracks at the entrance to the tracker, and their residuals to truth (bottom). 


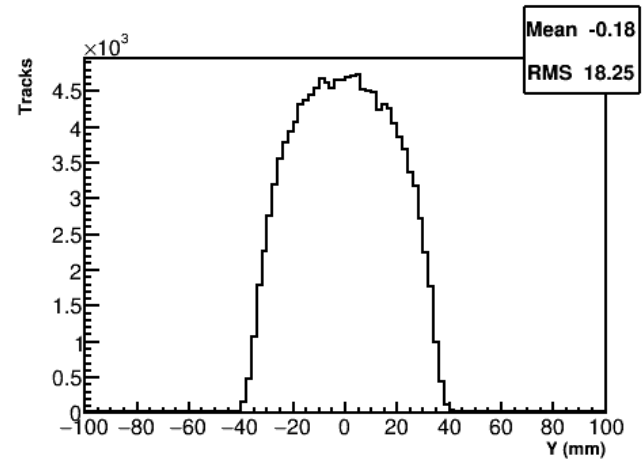

(a) Vertical position distribution. This distribution is bounded by the vertical extent of the tracker entrance.

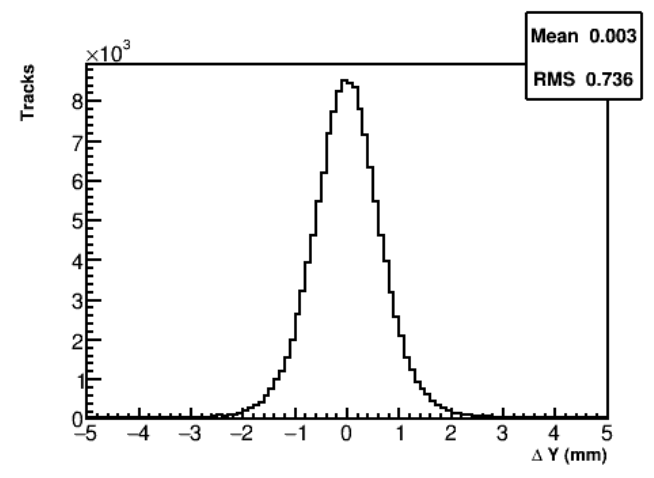

(c) The vertical position residual, which has a width of $736 \mu \mathrm{m}$ at the entrance of the tracker. This is larger than the horizontal position residual since the straws measure mostly in the horizontal plane.

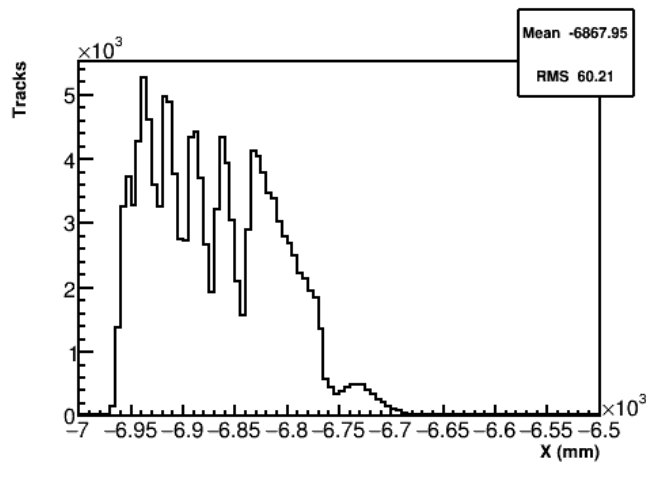

(b) Horizontal position distribution. The shape of the distribution is due to the acceptance of the tracker. It is perhaps more informative to look at the radial position distribution, which is shown later in Figure $4 \cdot 20$.

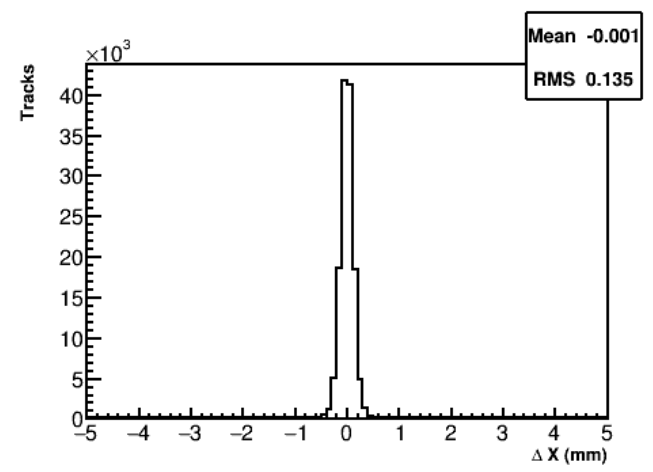

(d) The horizontal position residual, which has a width of $135 \mu \mathrm{m}$ at the entrance of the tracker. This is comparable to the input straw resolution of $150 \mu \mathrm{m}$.

Figure 4.17: Shown are the fitted vertical and horizontal position components (top) for tracks at the entrance to the tracker, and their residuals to truth (bottom). 


\subsubsection{Left-right ambiguity and fit modes}

Before actual data can be fit, the left-right ambiguity problem needs to be dealt with. Since straws only measure drift circles and not actual U or V positions, and since tracks are in general passing forwards through the detector, there is an ambiguity as to which side of the wire a particle passed for each hit. If even a single left-right choice for a hit is wrong then a track will most likely fit poorly. There are a couple of different track-fitting modes used to deal with this problem, which are detailed in Reference 89 and summarized here and in Reference 97 .

wireFit

The hits of a track from data are first fit to the wire centers which requires no leftright or drift-time information, simply called a wireFit. The errors on the measured hits are set as the RMS of a uniform distribution with a width of a straw diameter. The wire fit produces an approximation of the best fit track as well as some left-right information. The number of fitting iterations is capped at three for the wire fit.

mainFit

The primary fit mode for analyzing data is called mainFit. After the wire fit is completed, the predicted track parameters at each measurement plane are compared to the locations of the wires for the straws that were hit. The left-right choices for each hit are set depending on which side of the wire the predicted track passed. The measured hit positions and errors are updated from the wire values to the angularcorrected DCA values, based on the the left-right choices of each hit, and the fitting is repeated. At each iteration of the fitting, the left-right choices for each hit are updated based on where the previous predicted track parameters ended up. mainFit is the fastest fit mode and with this method about $66 \%$ of tracks fit well. The advantages in processing speed outweigh the lost track fraction as the tracking analyses are not 
statistics limited, and the lost tracks do not create a bias.

\section{fullSeqFit}

The secondary fit mode is called fullSeqFit. This fit mode does a better job of determining the left-right choices for the hits in a track, but is much slower than mainFit. Here is given a short summary; see Reference [89] for more technical details.

After a wireFit, the geometry of the straw layers is used to resolve some of the left-right choices for hits on a track. The left-right ambiguity is partially resolved through the shift in layers for each view as detailed in Section 3.3, where if a particle passes straight through both layers in a view it can be seen to go to the left of one wire and the right of the other. This implies that for curved tracks with certain angles through tracker modules, the left-right choices for some hits are known precisely [98]. For these hit planes the left-right choices are fixed for the rest of the fitting.

For the remaining hits, where the left-right choices are unknown, an approximate $\chi^{2}$ is calculated for each combination of left-right choices, $2^{N_{\text {unknown }}}$. This $\chi^{2}$ is calculated using the same formula as in the full fitting with the measured parameters set to the different left-right combinations, Equation 4.26. However, in this calculation the Geane transport and error matrices calculated by the wireFit are used instead of from a fit to the measured parameters with left-right choices. Since the calculation of these $\chi^{2}$ 's for every combination is slow, this process is sped up by limiting the left-right combinations to only $\mathrm{U}$ or $\mathrm{V}$ hit layers at a time, $2^{N_{\mathrm{U} \text {,unknown }}}+2^{N_{\mathrm{V} \text {,unknown }}} \ll 2^{N_{\text {unknown }}}$. This $\chi^{2}$ calculation produces a measure of how good a left-right combination is without having to propagate every track in Geane for the full fitting. The tracks with the best combinations and smallest approximate $\chi^{2}$ 's are then fit with the full Geane propagation and $\chi^{2}$ minimization algorithm. Typically the best 10 to 15 combinations are fit, and the final track with the best $\chi^{2}$ is taken as the best fit track. This approach determines the left-right choices for the fitted tracks correctly about $99 \%$ of 
the time with simulated data, but is very slow due to the number of combinations to check and the Geane propagation for each of the best track left-right combinations. If the track-fitting can be sped up significantly or a majority of the left-right choices constrained upstream somehow, then this fit mode would become the default.

\subsubsection{Fits to tracks from data and comparison with Monte-Carlo}

Fits to data are done with mainFit. Due to imperfect left-right assignments and the use of real DCA and DCA error measurements, the tracks from data are naturally more messy. See Figure $4 \cdot 18$ for a sample fitted track and a plot of the goodness-of-fit for the tracks. While not all tracks are fit perfectly, enough of them fit sufficiently well that the results can be passed to the extrapolation stage. Fitted tracks to Run 1 data are compared to results from Monte-Carlo. Track times and fitted total momenta are shown in Figure $4 \cdot 19$. Fitted track radial and vertical momentum and position distributions are shown in Figure $4 \cdot 20$. In general the two results are very similar. There are mismatches in the distributions because the idealized simulation did not reflect the real run conditions at the time of this track-fitting analysis. As described in Section 2.8 and explored later in Section 4.4, the muon beam was imperfectly stored in the ring, leading to the measured distributions here. 

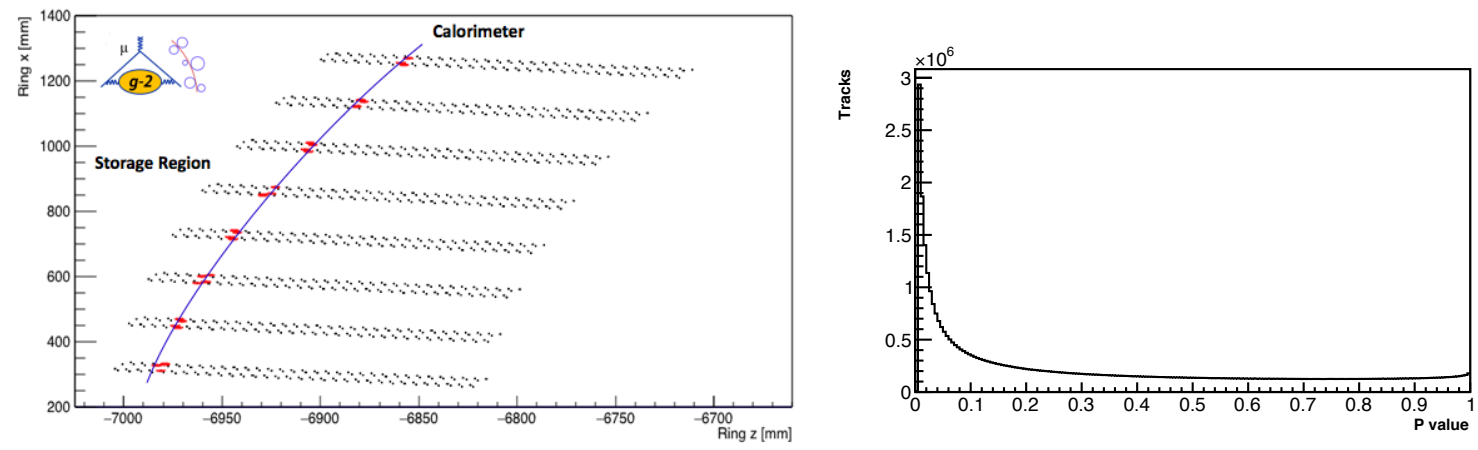

Figure 4.18: Sample fitted track (left) and p-value distribution (right) in data. The peak in the distribution at 0 arises from imperfectly fit tracks. A cut has been made in the p-value distribution at $1 \%$ to remove tracks which have entirely failed the fitting.

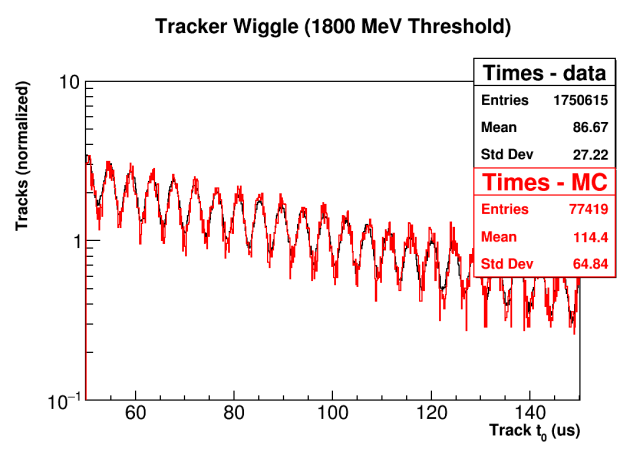

(a) Track times for tracks with energy greater than $1.8 \mathrm{GeV}$. The $\omega_{a}$ frequency can be seen in both the data and simulation.

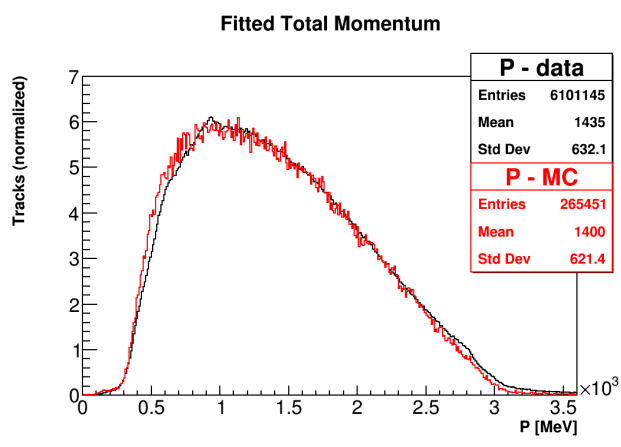

(b) Fitted track total momentum; there is a very slight mismatch between data and Monte-Carlo.

Figure 4.19: Fitted track results in data (black) vs Monte-Carlo (red). The number of entries in each are normalized so that they can be compared. Shape differences between the two are due to a mismatch between simulation conditions and the real experiment, and not any problem with the track-fitting. 


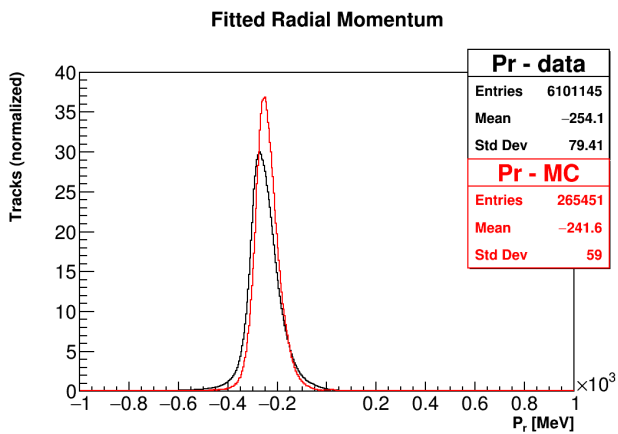

(a) Fitted track radial momenta. The radial momenta of the tracks are negative as particles are curving inwards through the tracker. There is a very noticeable difference between data and Monte-Carlo results.

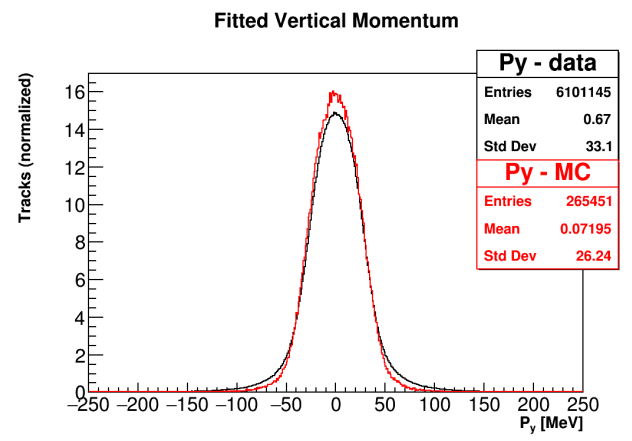

(c) Fitted track vertical momenta. The momentum is bounded by the storage region, and distribution from data is slightly wider than that from Monte-Carlo.

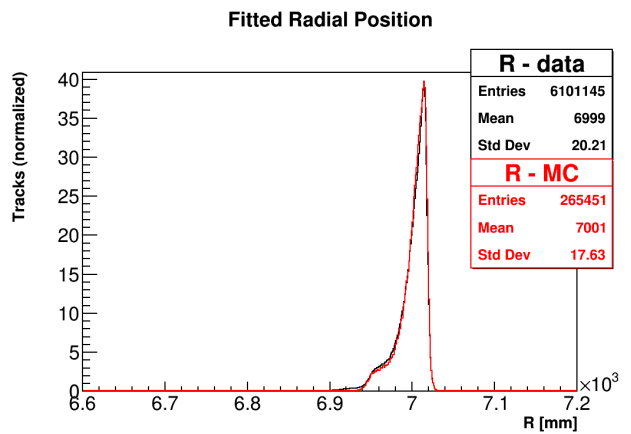

(b) Fitted track radial positions. The shape depends on the acceptance of the tracker and in general lies below the magic radius of $7.112 \mathrm{~m}$. Data and Monte-Carlo results match well.

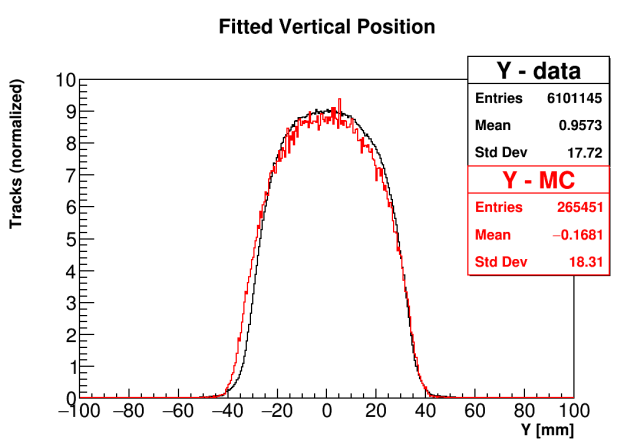

(d) Fitted track vertical positions. The positions are bounded by the tracker vertical acceptance. There is a noticeable difference between data and Monte-Carlo, with the data higher due to residual radial magnetic field in the experiment.

Figure 4.20: Fitted track results in data (black) vs Monte-Carlo (red). The amount of entries in each are normalized to each other so that they can be compared. Shape differences between the two are due to a mismatch between simulation conditions and the real experiment, and not any problem with the track-fitting. 


\subsection{Track extrapolation}

The last stage of the track reconstruction is the track extrapolation. The extrapolation takes the fitted track results and either extrapolates them back to the storage region, to the approximate position of the muon decay point, or forwards to the face of the calorimeter which sits right behind the tracker. The extrapolation stage utilizes a fourth order Runge-Kutta Nyström algorithm [80] which discretely steps a trajectory through the magnetic field in the full $g-2$ Geant4 simulation, similar in some respects to the error propagation. At each step of the extrapolation, the updated track

position and its error are compared to physical volumes in the simulation to flag tracks which have been reconstructed as likely originating from outside the storage region [80, 99]. Because there is no fixed interaction point in the storage region, tracks are extrapolated backwards to the point of tangency where the radial momentum is equal to zero. Monte Carlo studies verified that with a simple $1.1 \mathrm{~mm}$ correction at all momenta, the radial decay position could be adequately reconstructed using this approximation [80]. A schematic diagram explaining this behavior is shown in Figure $4 \cdot 21$. The vertical extrapolated distribution was found to have no biases. A bird's eye view for tracks extrapolated back into the storage ring is shown in Figure $4 \cdot 22$.

\subsection{Muon beam measurements}

All stages of the track reconstruction have ultimately facilitated the goal of determining the characteristics of the muon beam. As described in Section 2.6, the muon beam is not a fixed entity. Where the muons live and how they move in the storage region ultimately affect the calculation of the E-field and pitch corrections, the weighting of the muon distribution with respect to the measured field, and the precession frequency analysis based on data from the calorimeters. Presented here are plots for the $60 \mathrm{~h}$ dataset which describe the muon beam in the storage region. Analysis 


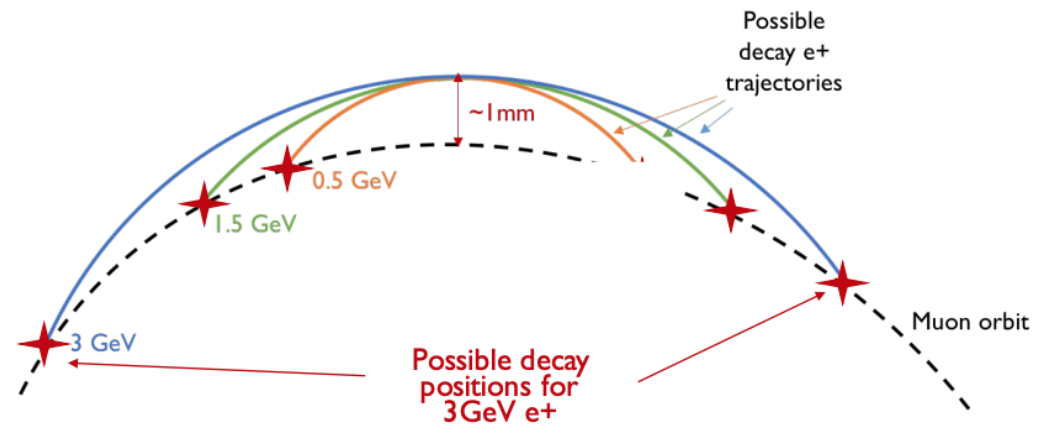

Figure 4.21: Tracks are extrapolated backwards into the storage region to the point of radial tangency. While for a single decay positron the extrapolated muon decay point will be different than the truth, the degeneracy of inwards and outwards decays leads to a correct average extrapolated position, with an applied radial offset of $1.1 \mathrm{~mm}$ as shown. High and low momentum tracks are emitted at smaller and larger angles respectively. Because high momentum tracks travel farther than low momentum tracks before reaching radial tangency, the offset is momentum-independent on average. Plot courtesy of S. Charity [80].

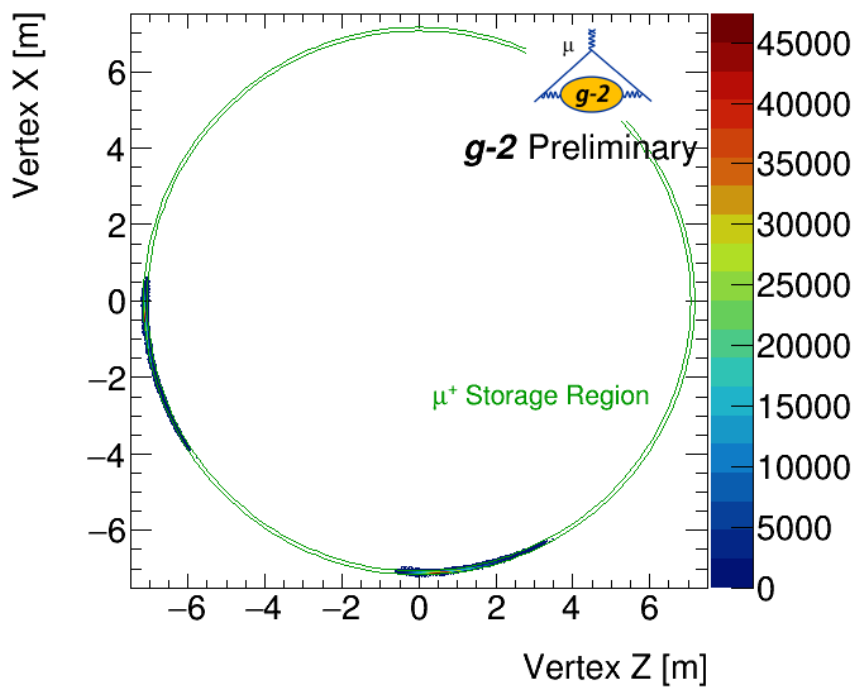

Figure 4.22: A bird's eye view of the extrapolation results in the storage ring. The two distributions of extrapolated tracks can be seen at the left and bottom of the figure, where the trackers sit at the head of each distribution. It can be seen that some tracks are extrapolated several meters back through the storage region. 
of the muon beam dynamics comes from the entire tracker group, with many plots having been provided by J. Mott. The analysis of acceptance differences between the tracker and calorimeter, which is critical for describing the correct muon distribution and dynamics in regards to those decay positrons which hit the calorimeters and are responsible for the measurement of $\omega_{a}$, is ongoing. Tracker-calorimeter matching studies will not be detailed here. A preliminary analysis is given in Reference [84.

Before characterizing the muon distribution, a set of quality cuts are applied to each track in order to remove failed or poorly fitted tracks. By applying these cuts, a clean sample of the extrapolated muon decay positions is extracted without biasing the results. The following conservative cuts are applied 100, 101:

- Successful track reconstruction and extrapolation

- No material hit during extrapolation back to vertex

- Number of tracking planes hit $\geq 12$

- p-value $>5 \%$

- Vertical extrapolation uncertainty: $0.5<\sigma_{y}<3.5 \mathrm{~mm}$

- Radial extrapolation uncertainty: $0.5<\sigma_{r}<5 \mathrm{~mm}$

- Horizontal entrance into tracker: $60<X<150 \mathrm{~mm}$

- Vertical entrance into tracker: $-40<Y<40 \mathrm{~mm}$

- Drift time: $0<t_{d}<70 \mathrm{~ns}$

- Track UV residuals $<500 \mu \mathrm{m}$

- Fraction of missed planes $<30 \%$

- $\mid$ Number of $\mathrm{U}$ hits - number of $\mathrm{V}$ hits $\mid \leq 4$ 


\begin{tabular}{lccccc}
\hline \multicolumn{3}{c}{ Run 1 Dataset Muon Beam Parameters } \\
\hline \hline Dataset & Mean & Peak & Width & Mean & Width \\
\hline 60h & 7.61 & 18.41 & 18.43 & 0.15 & 12.88 \\
HighKick & 6.89 & 15.95 & 17.74 & 0.11 & 12.92 \\
9d & 8.03 & 19.31 & 18.37 & 0.40 & 12.71 \\
Endgame & 7.40 & 18.11 & 18.01 & 0.95 & 12.68 \\
\hline
\end{tabular}

Table 4.1: Muon beam widths and means for the Run 1 precession frequency analysis datasets, where data has been combined from both tracker stations. Both radial and vertical values are defined with respect to the center of the storage region. The radial peak values were determined by fitting Gaussians around the peaks of the respective radial distributions. All values are in units of $\mathrm{mm}$.

First, a radial slice, or cross-section at a specific azimuth, of the extrapolated beam distribution is shown in Figure $4 \cdot 23$. The beam is off-center due to the lower than nominal kicker voltage used in Run 1 as described in Section 2.8. The radial and vertical projections of this cross-section are shown in Figure 4.24. The vertical distribution is very similar to that from the track-fitting results, while the radial distribution of the stored muons has a non-Gaussian shape due to the storage procedure of the muons. Because the beam is moving in time and these distributions include tracks of all times, these two distributions are generally wider than they are at a single point in time. Table 4.1 gives the measured values of the beam parameters corresponding to the various datasets for all times. Figure $4 \cdot 25$ shows the radial and vertical distributions of the muon beam as a function of time in-fill, with the means plotted in Figure $4 \cdot 26$. Because of the bad quadrupole resistors described in Section 2.8, the vertical center of the stored beam was higher than the center of the storage ring, and slowly moved downwards over the course of a fill; Figure $4 \cdot 27$. At the same time, the vertical width of the beam decreased slowly over the course of a fill. 


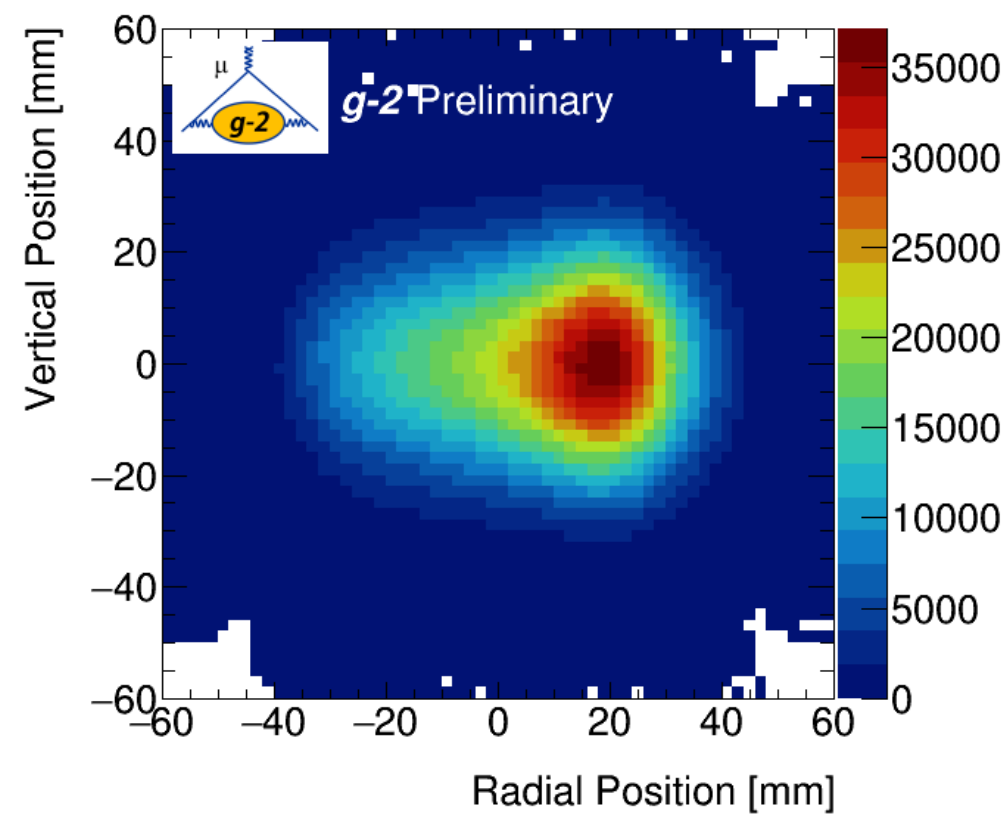

Figure 4.23: A radial slice of the extrapolated muon distribution or beam spot. The beam's radial center is greater than the magic radius because the integrated injection kick was less than optimal; Section 2.8 .

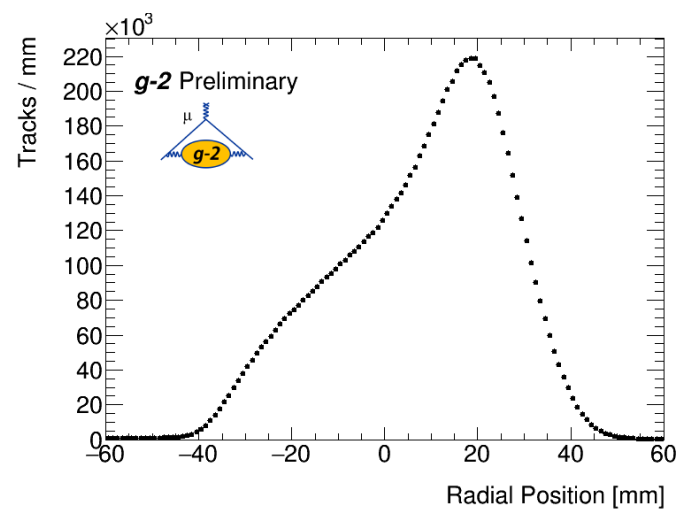

(a) The radial position of $0 \mathrm{~mm}$ corresponds to the magic radius of $7.112 \mathrm{~m}$. The radial distribution is peaked radial outward from the center.

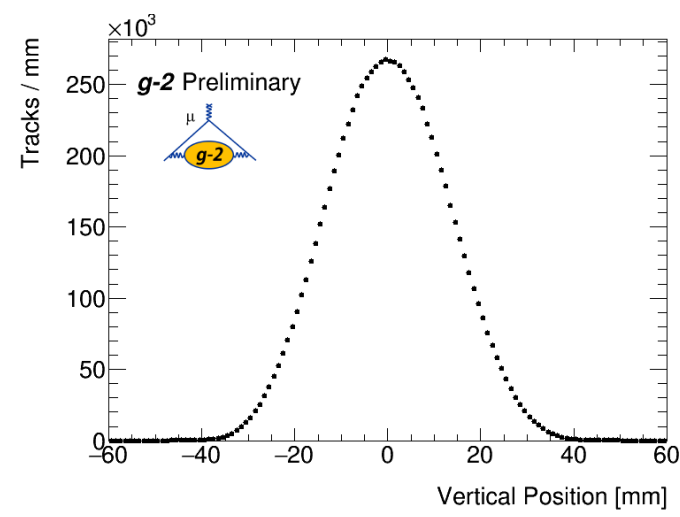

(b) The vertical distribution looks closely centered in the storage region, which has a width of $90 \mathrm{~mm}$.

Figure 4.24: Shown are the muon beam radial (left) and vertical (right) distributions for all track times. 


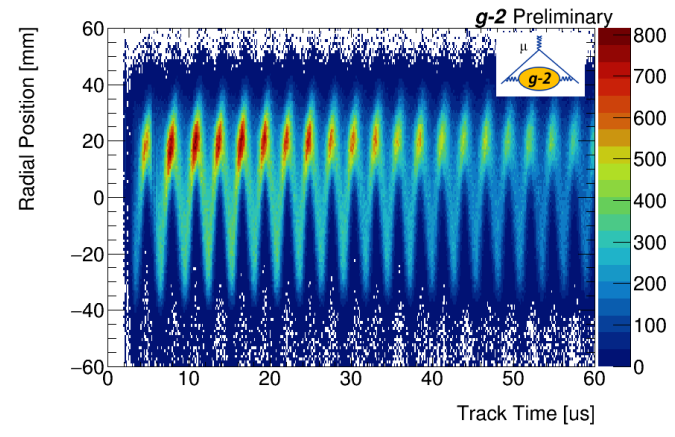

(a) The radial distribution of the muons oscillates radially inward and outward, and bunches and spreads out as a function of time. This behavior is the $\mathrm{CBO}$ of the beam.

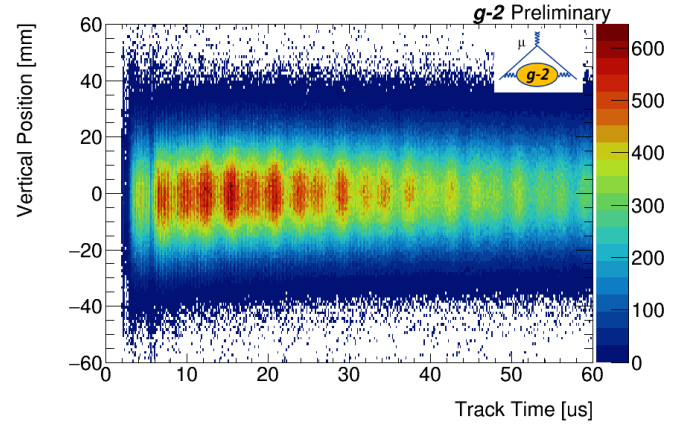

(b) The vertical distribution of the muons is relatively stable as a function of time, but there are oscillations in both the mean and width.

Figure 4.25: Shown are the muon beam radial (left) and vertical (right) distributions as a function of time in-fill for tracks in station 12 . In general the distributions for station 18 are very similar.

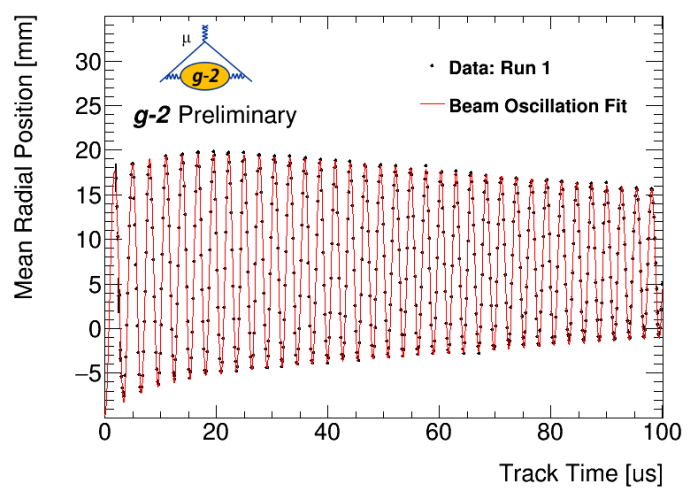

(a) The radial distribution mean oscillates at the $\mathrm{CBO}$ frequency. In station 12 the beam swings outward at early times due to the scraping procedure, and then slowly relaxes inwards.

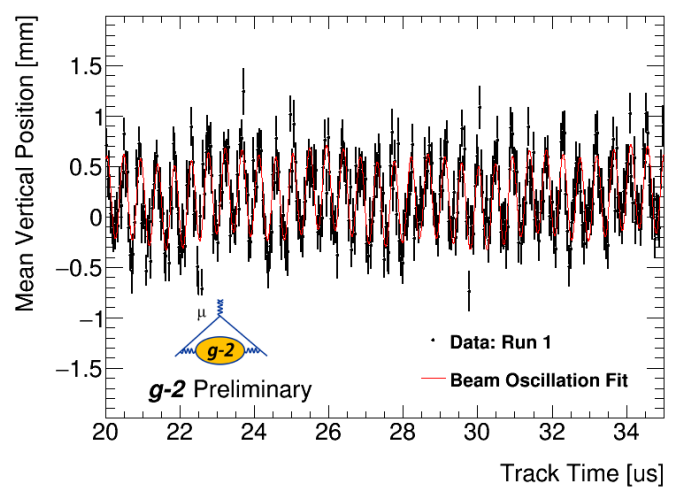

(b) The vertical distribution mean oscillates at the vertical betatron frequency, as described in Table 2.2. For these early track times the beam is actually stored slightly higher than the center of the storage region.

Figure $4 \cdot 26$ : Shown are the muon beam radial (left) and vertical (right) distribution means as a function of time in-fill for tracks in station 12 . In general the distributions for station 18 are very similar. 


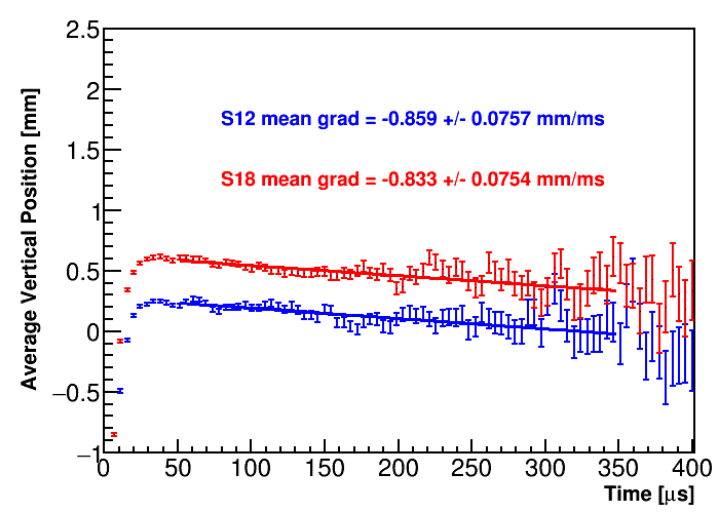

(a) Average vertical position.

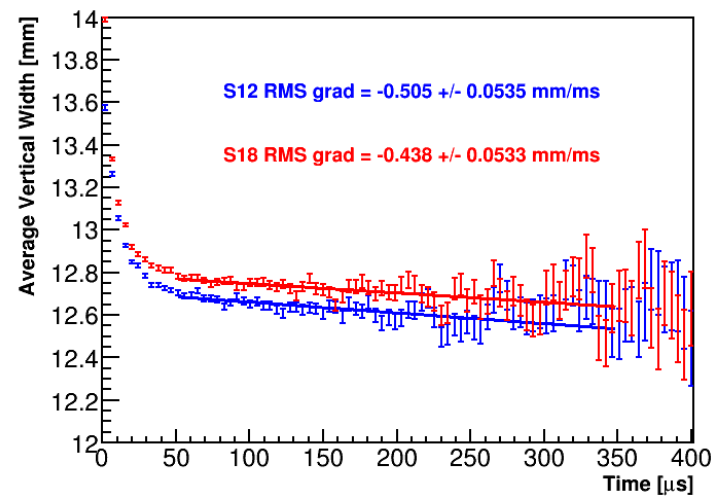

(b) Average vertical width.

Figure 4.27: Average vertical position (left) and width (right) as a function of time in-fill. The data points are binned such that the higher frequency betatron motion is binned out. Stations 12 and 18 are shown in the blue and red respectively. The sets of points correspond to the means and widths directly from histograms at specific time points. Gradients are calculated by fitting the points to a straight line from 50 to $350 \mu \mathrm{s}$. The vertical position of the beam drops over the course of the fill on the order of $1 \mathrm{~mm} / \mathrm{ms}$, depending on dataset, while the vertical width decreases on the order of $0.5 \mathrm{~mm} / \mathrm{ms}$. The differences between the two stations are due to the closed orbit. Plots courtesy of Joe Price. Fits were also done to means and widths calculated from Gaussian fits to the individual histograms with similar results [102]. 


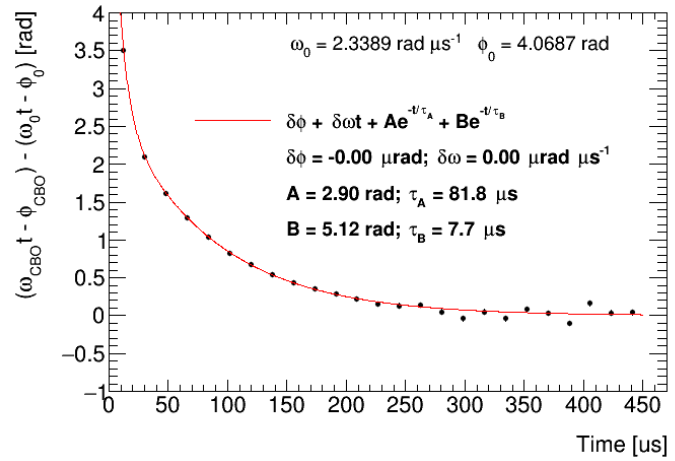

(a) The CBO frequency change is due to the slowly changing quad voltages over each fill. Plotted is the difference between the cosine argument for the changing frequency and the value it asymptotes to at late times. The frequency fit model includes two exponentials, one with a longer time constant and one with a shorter time constant.

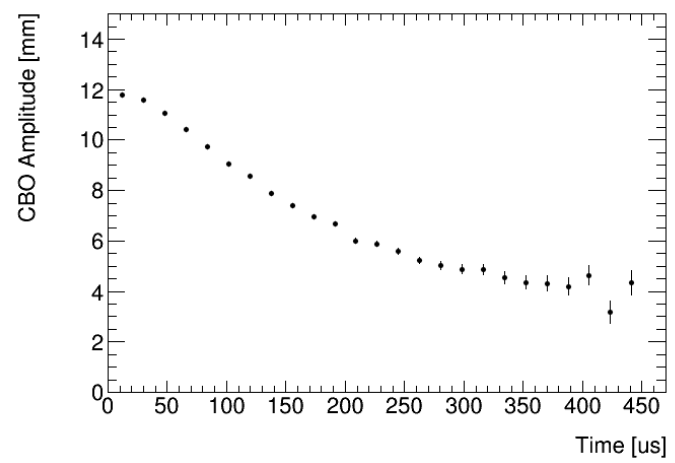

(b) The CBO effect decays away exponentially with a lifetime on the order of $200-$ 300 us. Reasonable CBO envelopes include a pure exponential or an exponential plus a constant.

Figure 4.28: CBO frequency (left) and amplitude (right) as a function of time in-fill.

The oscillation observed in the radial mean is the CBO described in Section 2.6.1 Using the tracking results shown here, the CBO frequency and amplitude can be characterized 103]. Because of the bad quadrupole resistors, not only does the average beam vertical position change over the course of a fill, but the CBO frequency changes as well. The CBO frequency and amplitude as a function of time in-fill are shown in Figure $4 \cdot 28$. It can be seen that over a course of a fill, the CBO amplitude and frequency decay away exponentially (where the frequency tends to its static value). Both of these characteristics of the beam motion are folded into the precession frequency analysis, as will be described in Chapter 5 . 


\section{Chapter 5}

\section{$\omega_{a}$ Measurement}

The $g-2$ frequency $\omega_{a}$ is measured by counting the number of detected positrons in the calorimeters above some energy threshold versus time, as described in Section 2.1. Doing so results in a histogram that is modulated by $\omega_{a}$, Figure $2 \cdot 4$. The $\omega_{a}$ analysis consists of the steps needed to construct and fit the decay positron time spectrum and the necessary related systematic error studies.

\subsection{Reconstruction of decay positron hits}

The calorimeters measure hit times and energies of incident particles, where these hit times and energies are determined by a reconstruction of the raw SiPM signals. In E989 there are two overall separate reconstruction algorithms, ReconWest and ReconEast, both written in the art framework just as the tracking reconstruction is. Each of these reconstruction algorithms is modularized such that the steps of the reconstruction process can be switched in and out at will. Using separate reconstruction methods instills confidence in results by removing single points of failure. The reconstruction method used in this analysis is ReconWest. A summary of its details will be presented here. A more thorough description is detailed by A. Fienberg in Reference 104.

The raw data are digitized waveforms of voltage traces from each SiPM for each calorimeter crystal. Due to the large amount of data coming in at the high sampling rate, only those pulses which exceed some threshold are saved to disk. Traces are 
checked against this pre-configured threshold by passing all of the data through a GPU farm in real time [105]. If any trace is found above threshold, then the data are saved from every SiPM in the hit calorimeter, for a time range around the overthreshold trace. This time range is called a time island, similar to that in the tracking reconstruction, and typically has a width of $40 \mathrm{~ns}$.

The traces are fit with templates in order to extract the area and peak times of any present pulses. Each SiPM has a set of templates for positrons and a set of templates for laser pulses, unique to that SiPM. These templates are extracted from data, where each template is determined by collecting many single pulse traces from a SiPM, normalizing by pulse area, aligning in time, and averaging them. These templates were checked against various systematic effects in order to verify that the constructed templates did not bias the time or energy measurements. These effects included hit angle, energy (pulse size), position, rate, and aging effects [77, 104]. To determine the time and energy of a hit, the SiPM trace is fit to it's corresponding templates using a $\chi^{2}$ minimization algorithm. In order to fit for multiple pulses in a single time island, the fitting procedure first fits with a single template, and then checks the residuals for any remaining peaks. If peaks above a threshold are found, then the fitting is repeated until all pulses have been fit. The algorithm's time measurements are unaffected by the number of pulses in a time island, and there is 100\% pileup separation at $5 \mathrm{~ns}$. See Figure $5 \cdot 1$ for a typical single template fit to a SiPM trace.

Once a pulse has been fit with a template, the pulse area needs to be converted to energy units using an energy calibration procedure. Several different techniques can be used, including a method that counts photo-statistics seen in the SiPMs. The default method is a comparison of lost muon energy signatures in the calorimeters. As described in Reference [106], muons lost from the storage ring can spiral inward 


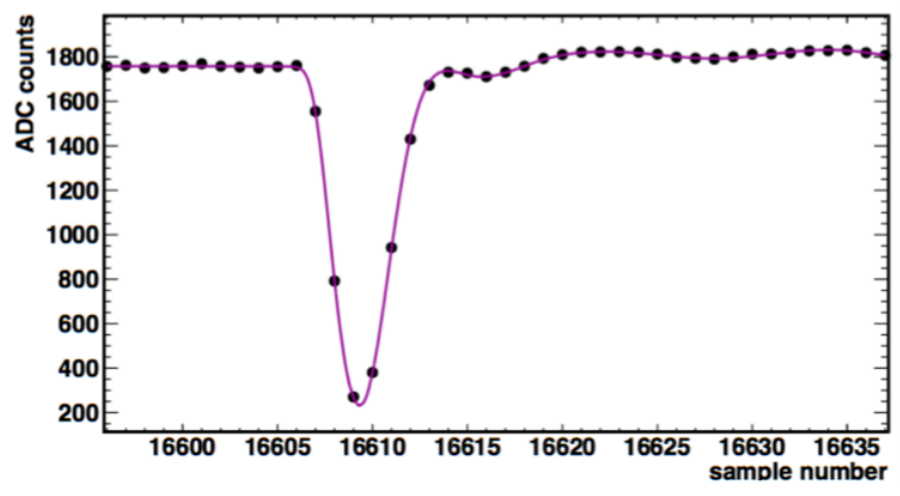

Figure 5·1: A template fit in purple to a SiPM trace delineated by the black points which are in units of ADC counts 104.

and hit consecutive calorimeters with a specific time separation between calorimeter hits. These lost muons are minimum-ionizing particles (MIPs), and thus leave a low energy signature in the crystals; see Section 5.3.3. Selecting on the time separation signature allows hits corresponding to lost muons to be isolated, and the peak of the energy signature can be used to determine the appropriate conversions from area to energy

The energy calibration for positron hits as compared to lost muon hits then needs to be determined. Again there are several different techniques, including a comparison of endpoint energies for high energy positrons which tail off at the magic momentum of $3.094 \mathrm{GeV}$, and comparison with simulation. The default technique is to calibrate the energies such that the optimal energy threshold for the $\omega_{a}$ analysis is near $1.7 \mathrm{GeV}$. This is justified from the fact that the lost muon energy signatures in different crystals should be the same regardless of SiPM gains and crystal properties after the correct gain constants have been applied. Ultimately the accuracy of the energy calibration is relatively unimportant because it is not the energy units that really matter. Rather, the quantity of interest is the number of positrons above some energy threshold, where that threshold can be optimized empirically. Indeed the entire $\omega_{a}$ analysis could be

\footnotetext{
${ }^{1}$ Different channels can also be equalized based on the energy signatures.
} 
done only considering the area of the SiPM pulses as a proxy for the energy of the incident positrons.

Each pulse now has an associated energy and time. The measurement of $\omega_{a}$ depends heavily on the time reconstruction since the analysis is a frequency extraction, thus the pulse times must be corrected for various effects in order to reach the precision goal. The fitted times for each pulse need to be aligned on a fill-by-fill basis relative to the injection time of the beam, corrected for any channel differences due to differing pulse shapes or optical fiber lengths, and corrected for any calorimeter time misalignments due to the use of different laser system components. The fill-byfill alignment is corrected using the T0 detector as described in Section 3.1.1. The calorimeter channels are aligned in time using signals from islands with large simultaneous pulses in neighboring crystals. Calorimeters are time aligned using lost muon coincident events as described before. Once the times of the pulse fits or crystal hits have been determined, the energies can be corrected for gain effects measured by the laser system. As described in Section 3.2.3, the laser calibration system corrects for out-of fill, STDP, and in-fill gain effects, in that order. Figure $5 \cdot 2$ shows an in-fill gain function fit to data for a single calorimeter crystal. Systematic effects from corrected gain variations are studied in Section 5.5.2.

The last part of the calorimeter reconstruction is the clustering stage, which assembles individual crystal times and energies into the times, energies, and positions of decay positron hits. For a time island with a single hit, the procedure is straightforward. The energy for the hit cluster is the sum of the individual hit crystal energies. The time for the cluster is taken as the time of the maximum energy hit in the island. This works because most of the deposited energy from a hit is localized to a single crystal. The position of the cluster is determined with a logarithmic weighting function between crystal hits, which for a $2 \mathrm{GeV}$ positron results in a resolution of 


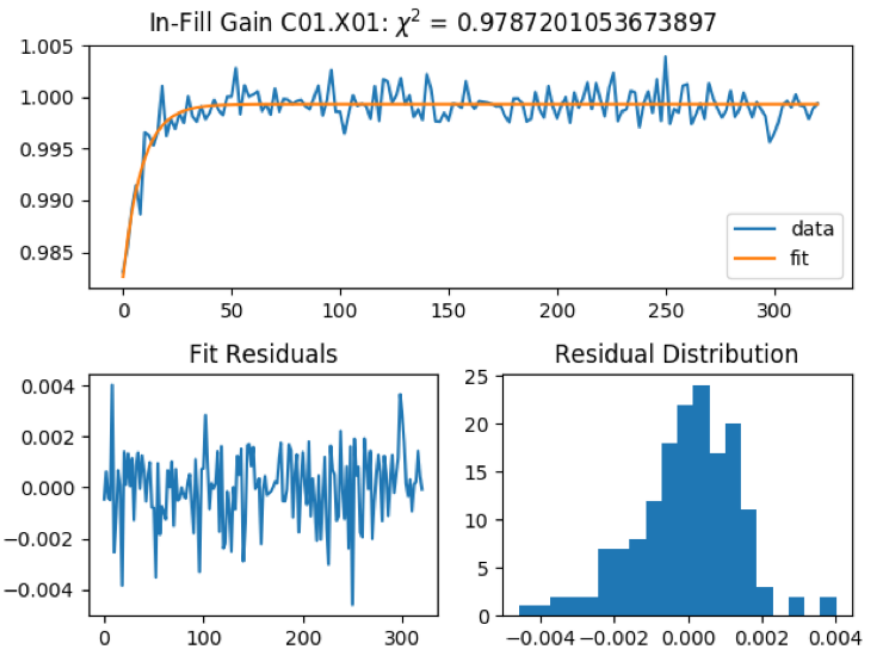

Figure 5·2: In-fill gain function fit for a single calorimeter crystal (top) and fit residuals (bottom). Each crystal has its own in-fill and STDP gain function parameters. Plot courtesy of Matthias Smith.

$2 \mathrm{~mm}$ [104]. Figure $5 \cdot 3$ shows a single cluster from a positron hit in a calorimeter. For a time island with multiple hits, the individual crystal hits are separated in time, where the time partitioning separates hits that are $2.5 \mathrm{~ns}$ apart, and the clustering proceeds as before. For hits which are within this time window, a pileup event has occurred which is considered as a single hit. If the pileup event happens within the same crystal, then the multiple hits are measured as a single hit, and this needs to be corrected for using a pileup subtraction technique, as described in Section 5.2.1. For hits that occur in separate crystals, the pileup can be resolved using the spatial separation of the calorimeters. This is an ongoing area of work, and one technique is described in Reference [104]. For this analysis the spatial separation was disabled. This increases the amount of pileup seen in the data which for the precision of the Run 1 analysis result was found to be acceptable. 


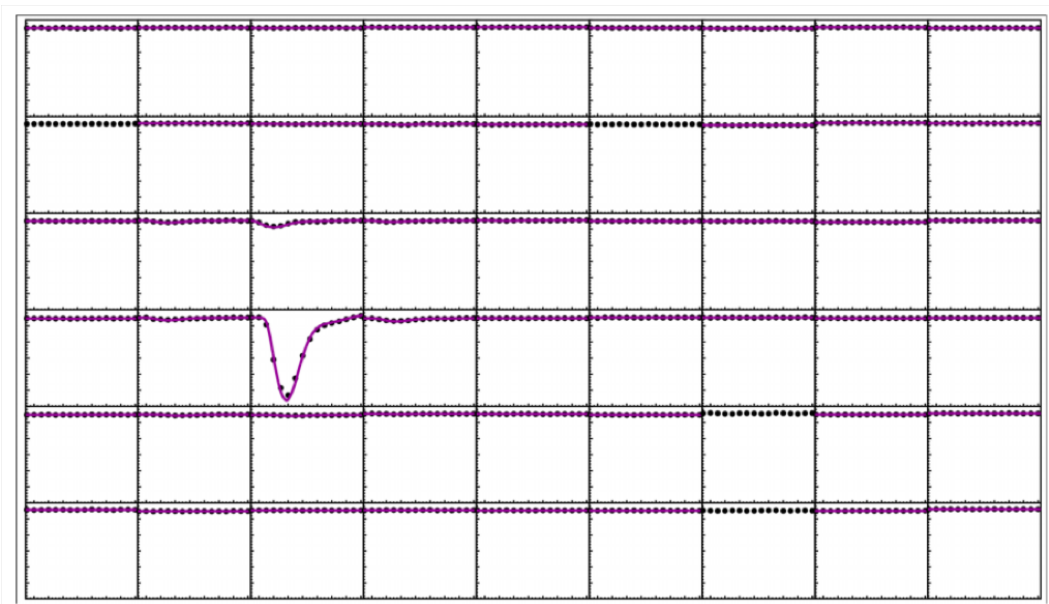

Figure 5·3: A single positron hit in a calorimeter, which resulted in a reconstructed calorimeter cluster [104]. Each box is a crystal in the calorimeter, where the contained trace is the SiPM output, fit with a template trace. The positron hit the crystal three crystals from the left and three crystals from the bottom, where it deposited most of its energy. Some of the energy was deposited in the neighboring crystals.

\subsection{Construction of positron hit energy and time spectra}

Once the reconstruction has processed all calorimeter hits into clusters, the energy and time spectra histograms are made. This procedure is typically analyzer dependent; here is given the procedure used in this analysis. At the very last stage of the reconstruction procedure, an art module takes the produced clusters and puts them into ROOT TTree formats, where individual data members include the energies, times, calorimeter numbers, etc. of the individual clusters. There are of order 20,000140,000 cluster data files per dataset, which are combined into order 200-1,400 ROOT TTree files. These ROOT TTrees are then passed through a ROOT macro to produce ROOT files with the histograms defined by the TH1F class, one ROOT histogram file per tree file.

It should be noted that some of the parameter choices for the constructed histograms were informed by analysis results. All analysis parameters were identical 


\begin{tabular}{lc}
\hline \multicolumn{2}{c}{ Time Spectra Parameters } \\
\hline \hline Parameter & Value \\
\hline Energy threshold $\left(E_{t h}\right)$ & $1700 \mathrm{MeV}$ \\
Bin width $\left(T_{c}\right)$ & $5 \mathrm{~ns}$ \\
Artificial dead time $(\mathrm{ADT})$ & $5 \mathrm{~ns}$ \\
Shadow dead time $(\mathrm{SDT})$ & $10 \mathrm{~ns}$ \\
Shadow gap time $(\mathrm{SGT})$ & 1 \\
Pileup energy scaling $(\mathrm{C})$ & $4.365411 \mu \mathrm{s}$ \\
$g-2$ period $\left(T_{a}\right)$ in Ratio Method & $64.44 \mu \mathrm{s}$ \\
Muon lifetime $\left(\tau_{\mu}\right)$ in Ratio Method
\end{tabular}

Table 5.1: Parameter values used in the construction of $\omega_{a}$ time spectra.

between the distinct analyzed datasets, in order to simplify both the comparison and combination of different dataset results. This section describes the justification for the choice of histogram parameters. A table of the histogram parameters is given in Table 5.1 .

Energy and time histograms are made for each individual calorimeter. These are summed together to form histograms of all hit times and energies. Figure $5 \cdot 4$ shows a sample energy spectrum for the Endgame dataset. An energy threshold is applied to the clusters before filling the time histograms. As described at the end of Section 2.1, the optimal energy threshold is where the quantity $N A^{2}$ reaches the maximum, at least in the case of a five parameter fit ${ }^{2}$. By scanning over energy threshold and fitting the resulting time spectra with Equation 2.13, the optimal energy threshold can be determined. The optimal choice of energy threshold was determined to be $1700 \mathrm{MeV}$, in accordance with the cluster reconstruction energy calibration; see Figure $5 \cdot 5$.

The optimal bin width for the time histograms was determined to be $149.2 \mathrm{~ns}$, the peak of the cyclotron period distribution determined from the fast rotation analysis

\footnotetext{
${ }^{2}$ Using the final fit function and looking at the error directly on the fitted $\omega_{a}$ frequency, a slightly better estimate can be found.
} 


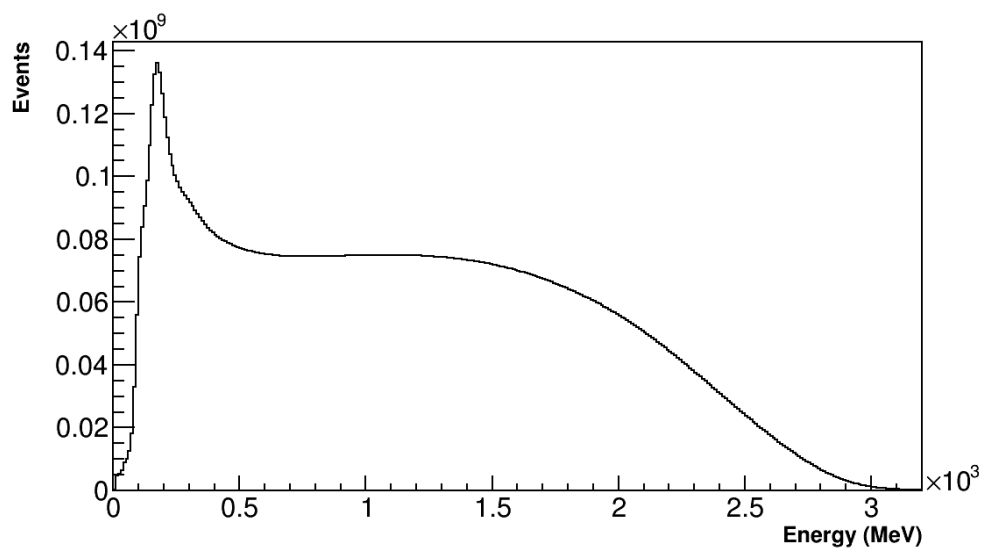

Figure 5·4: Energy spectrum for hits with cluster times greater than $25 \mu \mathrm{s}$ in all calorimeters on a linear scale. The peak at about $170 \mathrm{MeV}$ corresponds to lost muons laid on top of the low energy decay positron part of the spectrum. Data are from the Endgame dataset.

of the data. As described in Section 2.6.2, this bin width combined with a time randomization on each cluster over a range of $\pm T_{c} / 2=149.2 \mathrm{~ns} / 2$ serves to eliminate the fast rotation signal in the data ${ }^{3}$. This randomization is done using ROOT's TRandom3 class. As will be described in Section 5.3.2, the cluster times are also randomized by half the vertical waist period, $\pm T_{V W} / 2$, from Equation 2.26. Putting the frequency in terms of the field index $n$ and the cyclotron frequency $f_{c}$, the VW period is given by

$$
T_{V W}=\frac{1}{f_{V W}}=\frac{1}{(1-2 \sqrt{n}) \cdot f_{c}} .
$$

This randomization is done in order to remove the effects of the VW in the data The default random seed for each histogram ROOT file is the hash of the unique input

\footnotetext{
${ }^{3} \mathrm{An}$ alternative approach is to randomize all times in a single fill by half the cyclotron period as opposed to each individual pulse.

${ }^{4}$ Even though the VW frequency was found to be changing over the course of the fill, the change is small and the VW decays fast enough such that this constant time randomization was found to remove all residual traces of the VW in the data.
} 

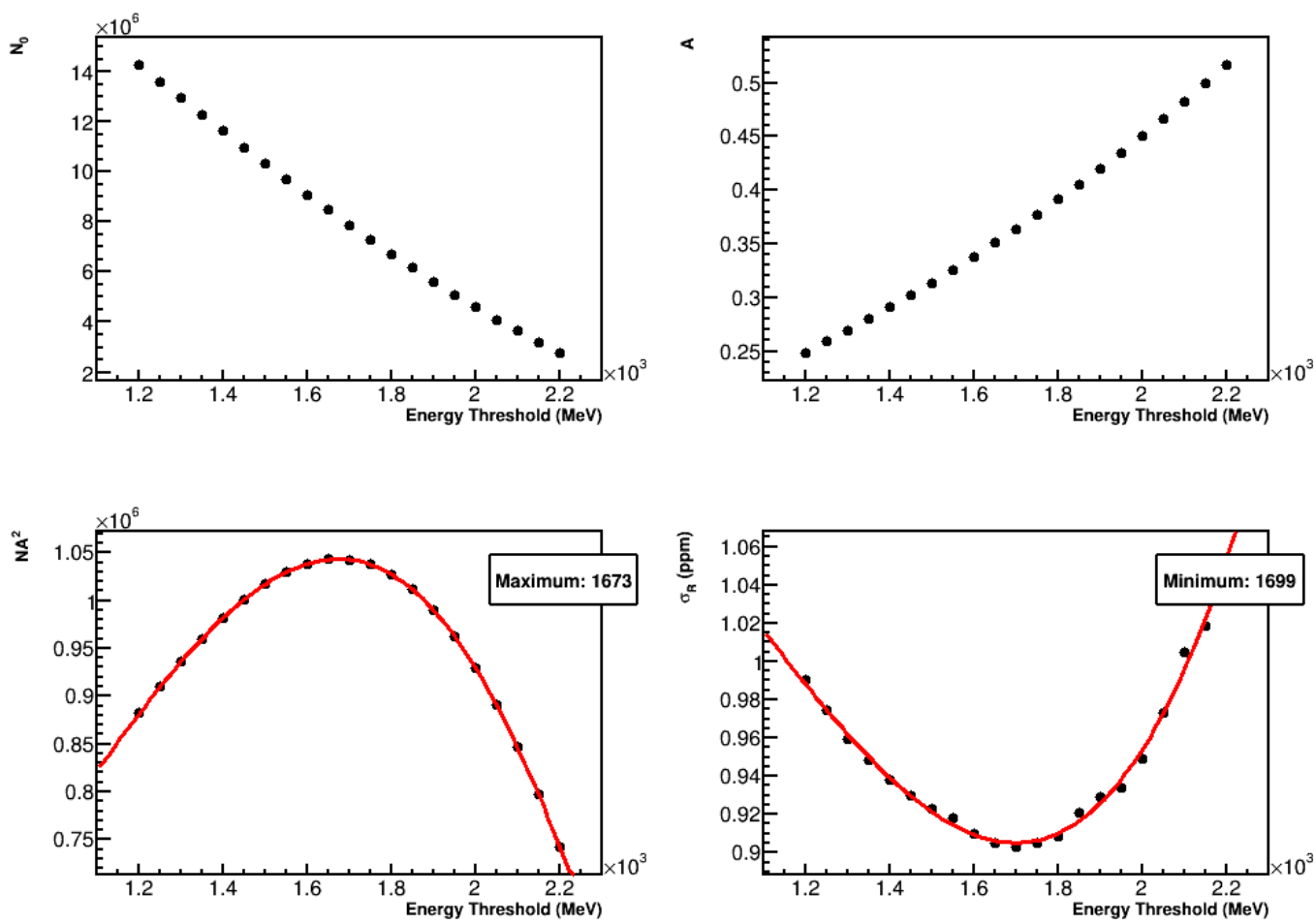

Figure 5.5: The optimal energy threshold can be determined from the $N A^{2}$ quantity as described in Section 2.1 from five parameter fits to the data (bottom-left) or from the calculated error with the final fit function (bottom-right). Fitted $N$ and $A$ parameters are also shown (top) which satisfy Equations 2.14 and 2.15. The maximum $N A^{2}$ and minimum $\sigma_{R}$ are determined using seven parameter polynomial fits to the respective quantities. The optimal threshold varies slightly per calorimeter and per dataset. Since the region of the minimum is relatively flat, a single energy threshold of $1700 \mathrm{MeV}$ was chosen. Data are from the $9 \mathrm{~d}$ dataset. 
file name using $\mathrm{C}++$ 's standard hash class. Other random seeds can be chosen in order to verify the consistency of fit results. Lastly, histograms are defined with a time range of $0-699.8972 \mu \mathrm{s}$ (the integer multiple of the bin width closest to $700 \mu \mathrm{s}$ ), corresponding to 4691 bins.

\subsubsection{Pileup subtraction}

As described in Section 3.2, there will be a certain amount of pileup in the detectors. Pileup distorts the measured energy and time spectra in a time-dependent way which is dangerous for the $\omega_{a}$ measurement. For the time histogram of clusters above energy threshold, the number of counts will be wrong for cases where two below-threshold positrons are registered as a single cluster above threshold, and where two abovethreshold positrons are registered as a single cluster. In the former, an extra count is added into the histogram, and in the latter a count is missed. The case where two lower energy positrons are registered as a single, higher energy cluster will have a different $g-2$ phase than an actual real single positron cluster at the same energy. This is because the lower energy positrons on average originate from muons which have traveled further around the ring, due to acceptance effects. These muons which have traveled further around the ring have spent more time in the magnetic field, and thus their spins have precessed more; see Figure 5.6. Clusters which originate from pileup events therefore have a different $g-2$ phase than non-pileup events.

If pileup was a constant effect in time, then the phase of the time histogram would be shifted by some constant amount, and the $\omega_{a}$ frequency extracted would be unaffected. However, the rate of pileup in the detectors changes over the time of a fill, as muons decay. The rate of pileup varies with the square of the instantaneous rate of positrons striking the calorimeters; double pileup events, which consist of events where two hits are registered as a single cluster, have a lifetime approximately half 


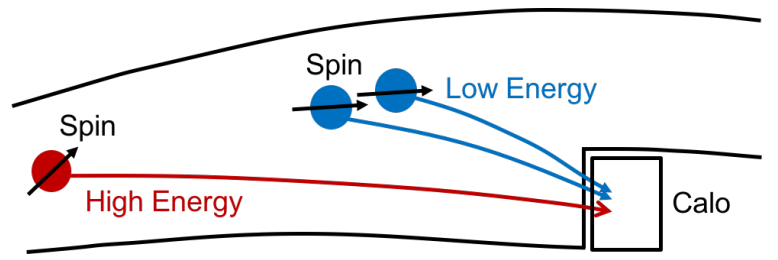

Figure 5·6: Pileup example, where two low energy positrons are registered as a single high energy positron. The black arrows indicate the (exaggerated) direction of the muon spins at the time of decay. Because of acceptance effects the lower energy decay positrons typically come from muons which have traveled further around the ring, and thus the muon spins have precessed more in the magnetic field, leading to a different measured $g-2$ phase for pileup events.

that of single hit events, and similarly for higher orders of the pileup effect5. Because the rate of hits in the detectors oscillates at the $g-2$ frequency, pileup will increase and decrease accordingly leading to oscillations in the pileup time spectra at $\omega_{a}$ and $2 \omega_{a}$. This time-dependent distortion means that the pileup effect needs to be included in the fit function or subtracted out of the data in order to extract the correct $\omega_{a}$ frequency. The former is challenging due to the non-linear nature of the dead time of the detectors, and would include another phase in the argument of the cosine term in the fit function, thus worsening the statistical precision of the extracted $\omega_{a}$ frequency. All analyzers thus construct an approximate pileup spectrum and subtract it from the data before fitting.

There are various methods to construct the pileup spectra. The method used in this analysis is called the 'asymmetric shadow method', used successfully in E821 [107]. This method statistically constructs an approximation of the pileup spectrum from the data by assuming that the probability of observing a pileup pulse, or doublet, is the same as the probability that two individual pulses (singlets) will be offset by some small amount of time, $\mathcal{O}(10 \mathrm{~ns})$. The method looks in time windows after trig-

\footnotetext{
${ }^{5}$ The true pileup spectrum is technically the convolution of the single hit time spectrum with itself, including effects from the non-linear dead time of the detectors.
} 


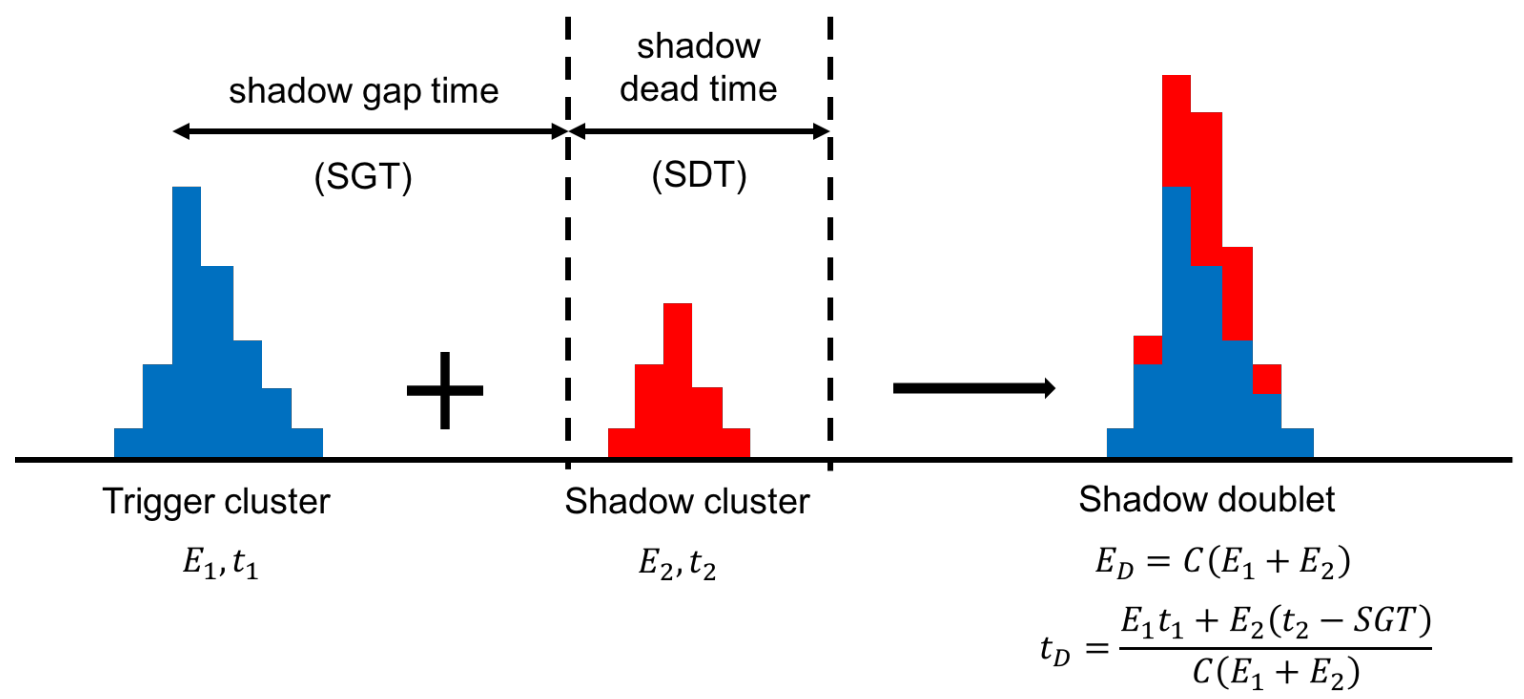

Figure 5·7: The shadow pileup method looks for shadow clusters within a time window SDT, a small gap time away (SGT) from trigger clusters. If a shadow cluster is found, an artificial doublet is formed and included in the pileup spectra if it exceeds the chosen energy threshold.

ger pulses to see if a 'shadow' pulse exists, and creates a 'shadow doublet' if so; see Figure $5 \cdot 7$.

The pileup spectrum $P$ is then the sum of all shadow doublets minus the singlets used in their construction, $P=D-S$. The width of the time window, and the time offset from the trigger pulse to the window, are called the shadow dead time (SDT) and shadow gap time (SGT) respectively. The times and energies of the constructed pileup doublets are taken as

$$
\begin{gathered}
E_{\text {doublet }}=C \cdot\left(E_{1}+E_{2}\right), \\
t_{\text {doublet }}=\frac{t_{1} \cdot E_{1}+\left(t_{2}-S G T\right) \cdot E_{2}}{E_{1}+E_{2}},
\end{gathered}
$$

where the energy of the doublet is the sum of the two singlet pulses $E_{1,2}$ times some calibration constant $C$, with a default value of 1 , and the time of the doublet is the energy-weighted time of the two singlets $t_{1,2}$. The procedure for constructing the pileup spectra is as follows: 
- Put each hit into a vector corresponding to a specific fill and a specific calorimeter

- Time order the hits

- Loop through the hits; for each hit look within a window of width SDT a time SGT later to see if a shadow pulse exists

- If a shadow pulse exists, construct a shadow doublet with an energy and time as defined in Equations 5.2 and 5.3

- Randomize $t_{\text {doublet }}$ over the range $\pm T_{c} / 2$ (to remove fast rotation as before, Section 2.6.2

- Per calorimeter, fill pileup energy and time spectra as $P=D-S$, where $D$ is the sum of doublets with positive weight in the histograms, and $S$ is the sum of all singlets used in the construction of the doublets, with negative weight. When filling the time spectra, set the times of the singlets to $t_{\text {doublet }}$, and only include those doublets and singlets above the energy threshold

In this way pileup energy and time spectra are constructed for each calorimeter, which can then be subtracted from the calorimeter cluster energy and time histograms. When combining the calorimeter data, the individual pileup histograms are added together before subtracting them from the calorimeter sum histograms.

The true dead time of the detectors depends on the energies and spatial separation of the incoming hits. In order to produce estimates of the pileup spectra which best match the data, an artificial dead time (ADT) is applied to the data before time randomization. While this slightly increases pileup, by applying an artificial dead time and matching the shadow window time such that SDT $=$ ADT, the pileup estimation is improved and the overall systematic error is reduced. The construction 


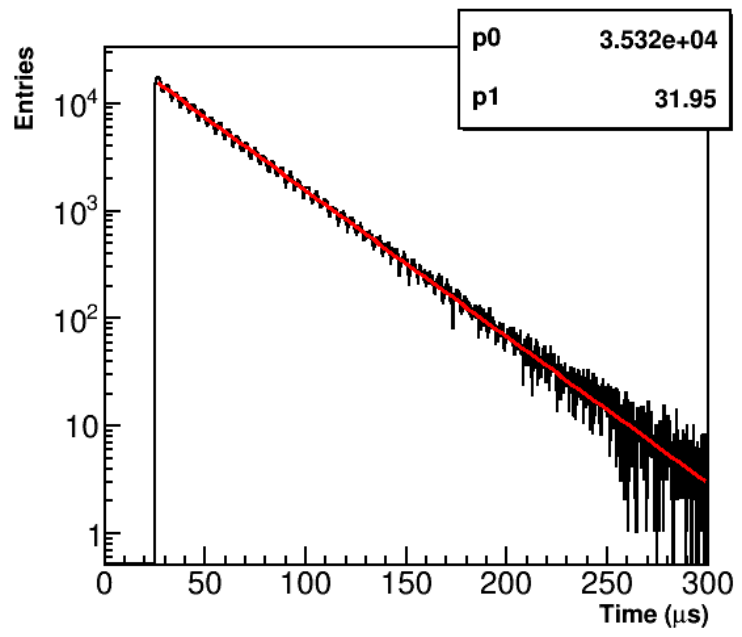

Figure 5.8: Plotted is the constructed pileup time spectrum on a log scale. Oscillations at $\omega_{a}$ can be seen by eye. The histogram is fit to a simple two parameter exponential to get an idea of the lifetime of the pileup, calculated here as $31.95 \mu \mathrm{s}$, which as expected is close to half of the muon lifetime at about $64.44 \mu \mathrm{s}$. Data are from the $60 \mathrm{~h}$ dataset.

of the artificial pileup is handled in the same way as the construction of the shadow pileup, with SGT set to 0 ns. The constructed artificial doublets replace the singlets in the data. The value for the ADT and SDT is set at $5 \mathrm{~ns}$, the time threshold at which pileup is $100 \%$ resolved.

The value of the SGT is simply set to twice the SDT, in order to push the shadow window out to times well beyond the dead time of any pileup events, but not so far that the probability of measuring a hit has appreciably changed. The value of the doublet energy scaling factor $\mathrm{C}$ is set to 1 , which is a fine approximation as the spatial separation in the reconstruction was turned off 5 . The values for each pileup parameter were shown previously in Table 5.1 .

The pileup time spectrum for those pileup pulses above the $1700 \mathrm{MeV}$ energy threshold is shown in Figure 5.8. The pileup energy spectrum and the cluster energy

\footnotetext{
${ }^{6}$ With the spatial separation turned off, 'pileup' events can occur in crystals that are easily separated by eye. While this increases the level of pileup seen in the data, the pileup approximation method also does not consider the spatial separation, and thus handles the level of pileup accordingly.
} 


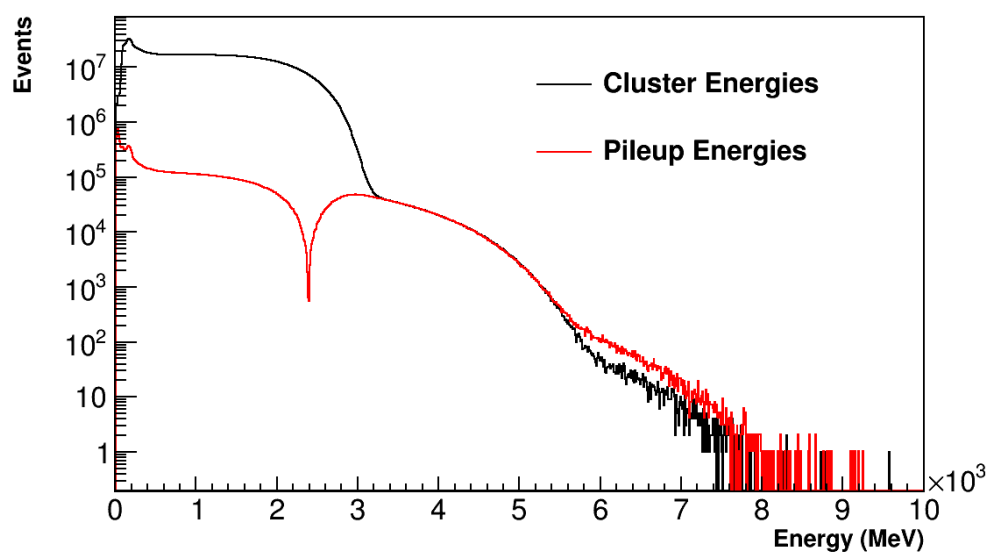

Figure 5.9: Cluster energies in black are plotted vs pileup energies in red, for all calorimeters added together, on a log scale. At energies below about $2.4 \mathrm{GeV}$ the pileup energy spectrum goes negative. In this plot the absolute value of the pileup energies is plotted, and a spike at about $2.4 \mathrm{GeV}$ can be seen as a consequence of this. The shapes do not match perfectly for the constructed pileup spectra, which can be seen at high energies. Data are from the 60h dataset.

spectrum are shown in Figure 5.9. In general, the two lobes starting at approximately $3 \mathrm{GeV}$ and $6 \mathrm{GeV}$ consist of double and triple pileup events respectively 7 . It can be seen that the shadow method of pileup construction produces a pileup energy spectra which is a good approximation of the cluster energies above the end point of a single decay positron, for cases of double and even triple pileup. The shape difference arises from two factors. First, the shadow method used in this analysis only constructs doublets, and does not consider cases of triple or higher orders of pileup. Second, the real pileup in the data contaminates the construction of the shadow pileup spectra, such that a shadow doublet can be constructed from real pileup pulses. While this alleviates the triplet problem slightly, it means that the doublet pileup spectrum is slightly wrong.

\footnotetext{
${ }^{7}$ All orders of pileup fill out the whole energy range, but certain regions consist of mostly one order of pileup or another.
} 


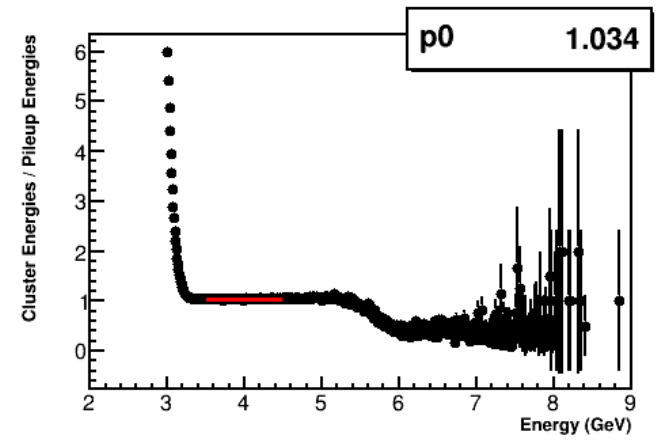

Figure 5·10: Cluster energies divided by pileup energies. A region from $3500-4500 \mathrm{MeV}$ is fit to a straight line, where the doublets dominate the energy distribution. Data are from the $9 \mathrm{~d}$ dataset.

In order to produce a slightly better estimate of the pileup, a multiplier can be applied to the pileup energy and time spectra. By taking the ratio of cluster energies over pileup energies and fitting the region where the energies are dominated by real pileup doublets, a correction factor of approximately $3-4 \%$ is found, as shown in Figure 5.10. The default multipliers for the Run 1 datasets are 1.03210, 1.03413, 1.03387, and 1.03819 for the 60h, HighKick, 9d, and Endgame datasets respectively. Similarly, the cluster time spectra can be examined for cluster energies over $3500 \mathrm{MeV}$, where the clusters consist purely of pileup pulses. By taking the ratio of the pileup corrected times over all times, the level of residual pileup can be determined. Just as in the ratio of the energies, an approximate 3-4\% factor is found. When applying this multiplier, the cluster times above $3.5 \mathrm{GeV}$ are eliminated as shown in Figure $5 \cdot 11$. As will be shown in Section 5.5.1, the scale of this multiplier is well within $1 \sigma$ of the pileup multiplier error. The corrected energy spectra (cluster energies minus pileup energies) after the application of this multiplier for the $60 \mathrm{~h}$ dataset can be seen in Figure $5 \cdot 12$. There is a small residual shape mismatch in the energy spectrum as the corrected energy spectrum does not lie exactly along 0 for energies greater than the endpoint energy of $3.094 \mathrm{GeV}$ plus the energy response of the detectors. 

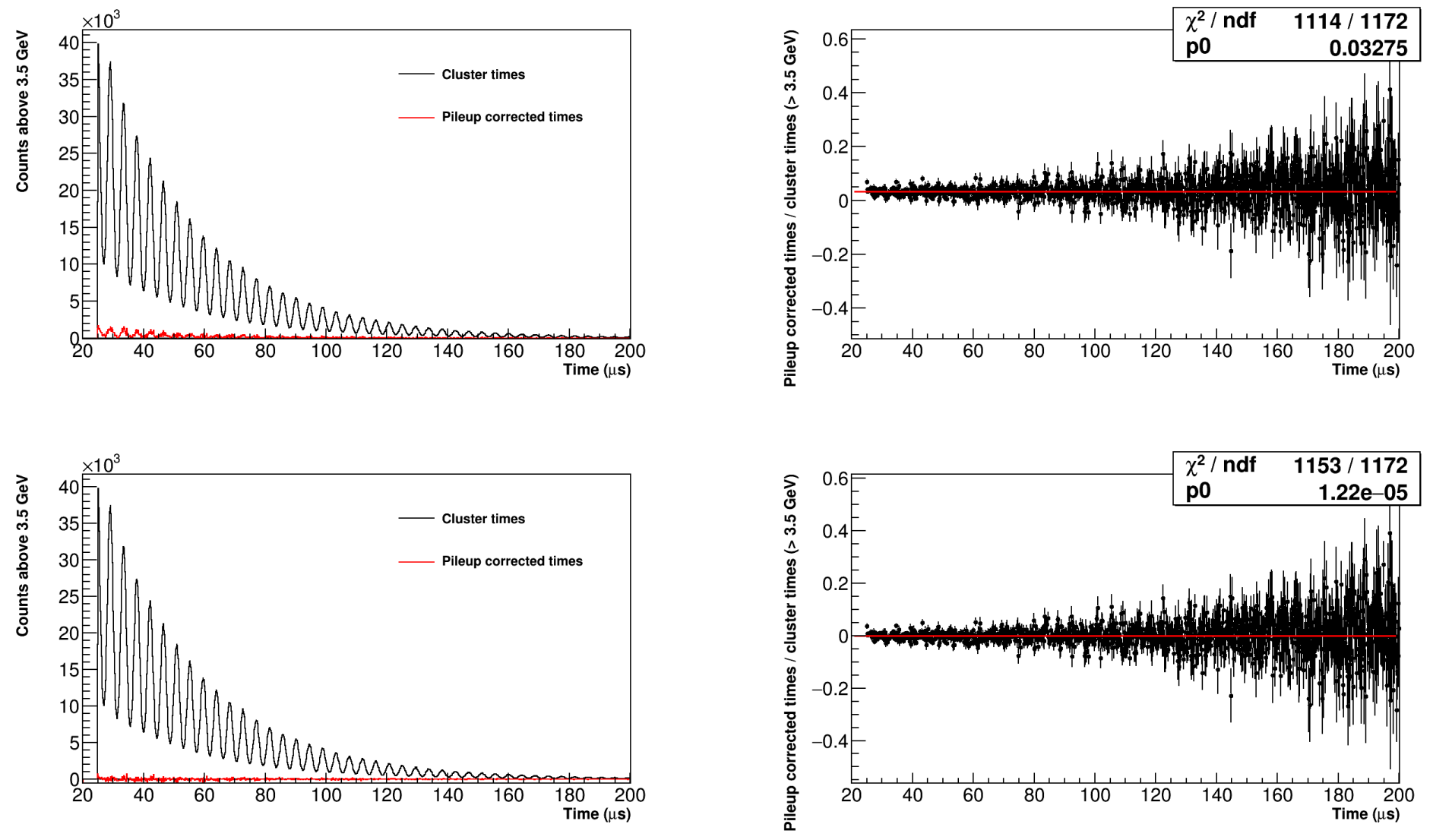

Figure 5·11: Cluster times and pileup corrected times for counts above $3.5 \mathrm{GeV}$ (left) and their ratio (right). The top two plots are used to determine the approximate level of residual pileup in the data, which is approximately 3\%. The bottom two plots show the application of that factor and the resulting removal of the remaining pileup. Data are from the $9 \mathrm{~d}$ dataset. 


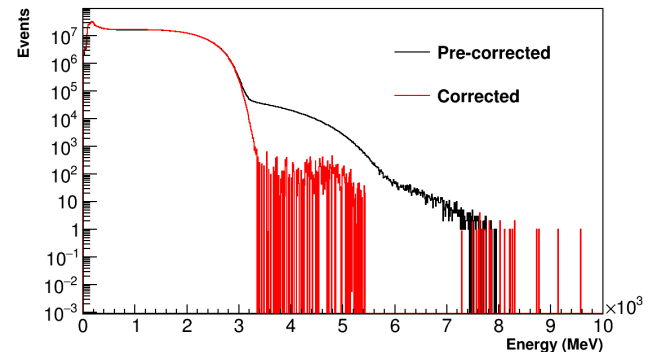

(a) Log scale.

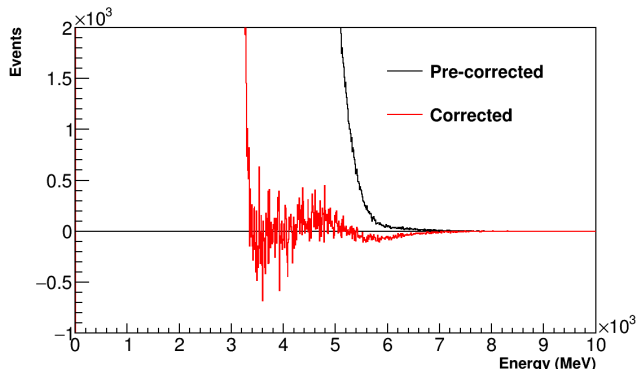

(b) Linear scale.

Figure 5.12: Plots for the pre-corrected and corrected energy spectra are shown, all calorimeters added together. Because the triplets and contamination are not accounted for, the corrected energy spectrum does not lie exactly along zero above the energy response of the detectors. Data are from the $60 \mathrm{~h}$ dataset.

It has been determined that regardless of any residual shape mismatch in the cluster times below $3.5 \mathrm{GeV}$ (implied to be small due to the lack of such a shape mismatch above $3.5 \mathrm{GeV}$ ), the systematic error on the extracted $\omega_{a}$ frequency due to the pileup correction procedure is within the target uncertainty for the level of statistics in the Run 1 dataset; see Section 5.5.1. For analyses after Run 1 where the error budget is reduced, it may be necessary to improve the shadow method to account for triplets and the contamination. Finally, since the pileup is statistically constructed and then subtracted from the data, the errors on the final time histogram are no longer pure Poissonian. The proper calculation of the errors is detailed in Appendix C.

\subsubsection{Ratio Method}

In this analysis, $\omega_{a}$ is extracted with the "Ratio Method," or sometimes "R-Method." It is a technique that modifies the data in such a way that the exponential decay in the time histogram is removed, and slowly varying and smooth effects are reduced. It was used successfully in the E821 experiment [108, 109, 110]. A full derivation of 
the equations in the method is given in Appendix $B$; here is given a short summary. Figure $5 \cdot 13$ provides a pictorial representation of how the method works.

The first step of the method is to randomly divide the data into four separate datasets, one with the times of all clusters shifted up by half a $g-2$ period, $+T_{a} / 2$, one with cluster times shifted down by half a $g-2$ period, $-T_{a} / 2$, and two unchanged. Assuming the data are described by the five parameter function described in Section 2.1 and shown in Figure $5 \cdot 13 \mathrm{a}$,

$$
N_{d}\left(t, E_{t h}\right)=N_{0}\left(E_{t h}\right) \cdot e^{-t / \gamma \tau_{\mu}} \cdot\left[1+A\left(E_{t h}\right) \cos \left(\omega_{a} t+\phi\left(E_{t h}\right)\right)\right]
$$

and that the data are equally split into four subsets, then the four new datasets are taken to $b e^{8}$,

$$
\begin{aligned}
& u_{+}(t)=\frac{1}{4} N_{5}(t+T / 2) \\
& u_{-}(t)=\frac{1}{4} N_{5}(t-T / 2) \\
& v_{1}(t)=\frac{1}{4} N_{5}(t) \\
& v_{2}(t)=\frac{1}{4} N_{5}(t)
\end{aligned}
$$

In order to time shift the data as such, $T_{a}$ needs to be known a priori to reasonable precision. The value used is

$$
T_{a} \approx 4.365411 \mu \mathrm{s}
$$

where it has been calculated from the E821 results as $T_{a}=1 / f_{a}$, where $f_{a}$ is $0.2290735 \mathrm{MHz}$. This value for $f_{a}$ was determined by averaging column two of Table XV of the E821 Final Report [36], which consists of the $f_{a}$ results for the different run periods in that experiment. A systematic error on the choice of this parameter

\footnotetext{
${ }^{8}$ When handling the pileup in the ratio method, the pileup time spectra are split into four datasets and time-shifted in the same way as the cluster hit times. Associated doublets and singlets are kept together in the same individual dataset, and the four pileup datasets are subtracted from their respective ratio datasets before forming the ratio.
} 
is calculated in Section 5.5.5 and is negligible for the precision known.

The datasets are then combined as

$$
\begin{aligned}
& U(t)=u_{+}(t)+u_{-}(t), \\
& V(t)=v_{1}(t)+v_{2}(t),
\end{aligned}
$$

both of which are shown in Figure $5 \cdot 13 \mathrm{~b}$. It is immediately apparent that the $U(t)$ data are shifted $180^{\circ}$ out of phase from the $V(t)$ data. The ratio is then defined as 9

$$
R(t)=\frac{V(t)-U(t)}{V(t)+U(t)}
$$

where the numerator and denominator are plotted in Figures $5 \cdot 13 \mathrm{c}$ and $5 \cdot 13 \mathrm{~d}$ respectively. The numerator is an exponentially decaying cosine, while the denominator is a simple exponential. Plugging the time-shifted five parameter function into the $U(t)$ and $V(t)$ variables, the ratio spectrum can be derived as

$$
R(t) \approx A \cos \left(\omega_{a} t\right)-C
$$

where

$$
C=\frac{1}{16}\left(\frac{T}{\tau}\right)^{2} \approx 2.87 * 10^{-4}
$$

As shown in Equation 5.9 and in Figure $5 \cdot 13 \mathrm{e}$, the exponential has been eliminated and the number of parameters in the fit function is reduced from five to three.

In addition to the exponential being eliminated, any slow or smooth terms in the data get time-shifted and divided as well, such that the amplitude of said effects are reduced. For faster effects, the degree of cancellation of the effect is dependent on the frequency. Effects at frequencies which are an odd multiple of $\omega_{a}$ are preserved while

\footnotetext{
${ }^{9}$ The ratio can also be defined with $U(t)-V(t)$ in the numerator. However, in that case the phase of the ratio spectrum is shifted $180^{\circ}$ from the original $N_{5}(t)$ spectrum.
} 


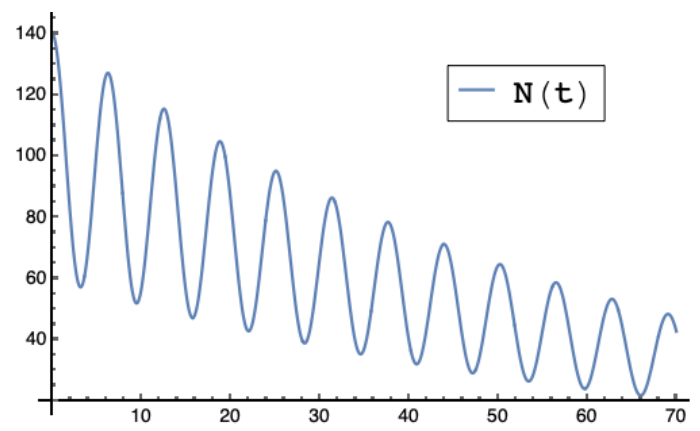

(a) The five parameter function, defined in Equation 5.4, describes the incoming data to first order.

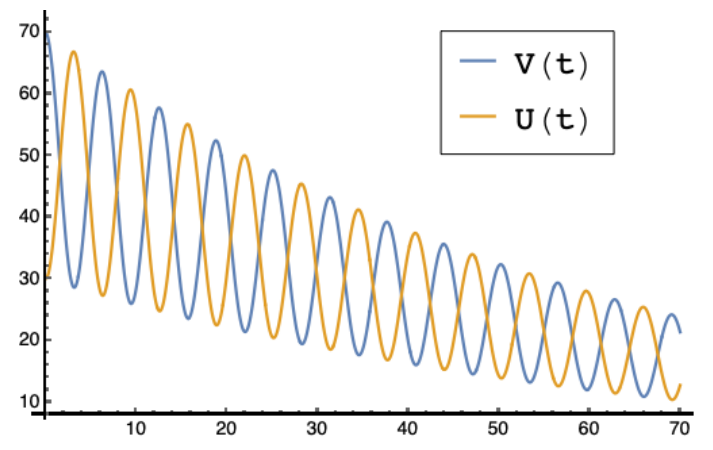

(b) $U(t)$ and $V(t)$ functions describe the time-shifted and un-shifted ratio datasets.

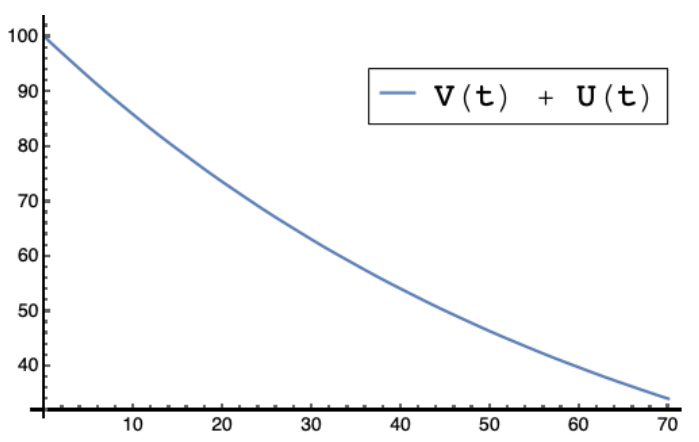

(d) The denominator function in the formed ratio, $V(t)+U(t)$, is a decaying exponential.

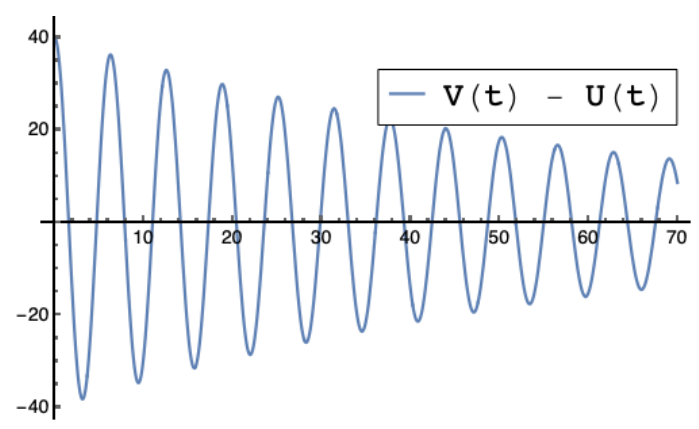

(c) The numerator function in the formed ratio, $V(t)-U(t)$, is an exponentially decaying cosine.

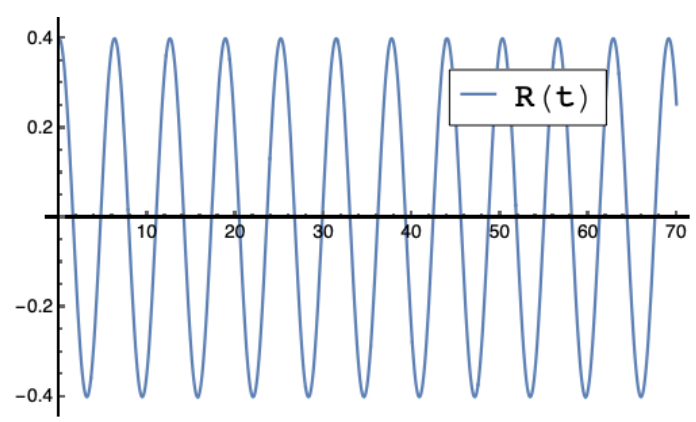

(e) The ratio function describes the data after it has been transformed according to the text. To first order it is a simple cosine.

Figure 5·13: Functions describing the formation of the ratio data. 
effects at an even multiple of $\omega_{a}$ are completely canceled out. An example is shown in Figure $5 \cdot 14$. While this amplitude reduction makes fitting the data easier in some cases, it can also be a downside when certain effects still need to be included in the fit function but are harder to do so due to their smaller signals.

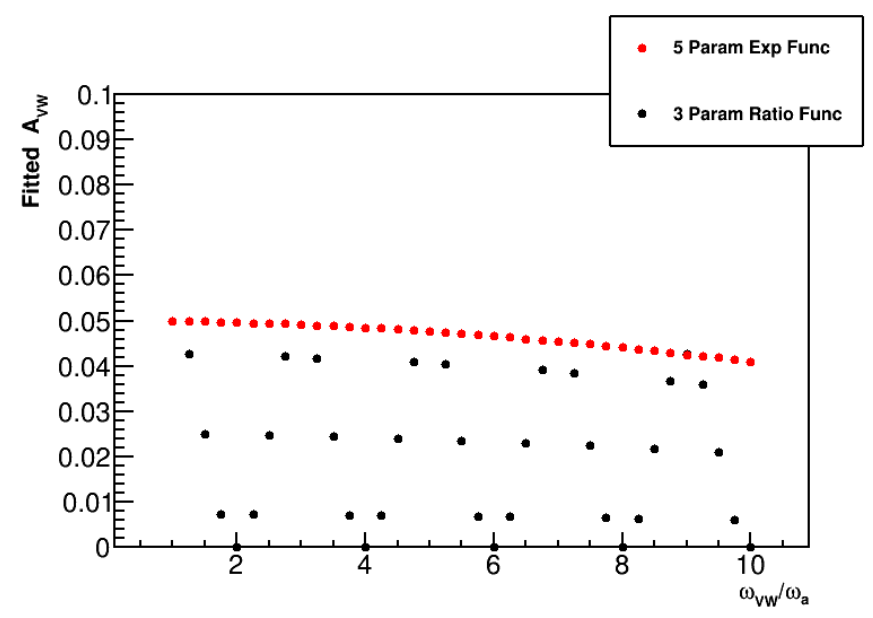

Figure 5.14: Fitted amplitude for a VW effect as a function of frequency in units of $\omega_{a}$ in a Toy MC simulation. Fit results with a five parameter function are shown in red and a three parameter ratio function are shown in black. The input amplitude is 0.05. The fall off of red points is due to the high frequencies relative to the bin widths leading to an underestimate of the amplitude; this is unimportant and performing an integral fit removes this trend. As shown, the amplitude of the effect in the ratio fit goes to zero for frequencies which are an even multiple of $\omega_{a}$.

In order to eliminate the constant $C$ at the end of Equation 5.9 , a different weighting scheme is used in this analysis as described in Reference [53]:

$$
\begin{aligned}
& u_{+}(t)=\frac{e^{T / 2 \tau}}{2+e^{T / 2 \tau}+e^{-T / 2 \tau}} N_{5}(t+T / 2) \\
& u_{-}(t)=\frac{e^{-T / 2 \tau}}{2+e^{T / 2 \tau}+e^{-T / 2 \tau}} N_{5}(t-T / 2) \\
& v_{1}(t)=\frac{1}{2+e^{T / 2 \tau}+e^{-T / 2 \tau}} N_{5}(t) \\
& v_{2}(t)=\frac{1}{2+e^{T / 2 \tau}+e^{-T / 2 \tau}} N_{5}(t)
\end{aligned}
$$


Here $\tau=\tau_{\mu}$, where $\tau_{\mu}$ is the time-dilated muon lifetime. The factors out front are each approximately $1 / 4$ and account for the effect of muon decay over a time period of $T_{a} / 2$. Similar to $T_{a}$, the muon lifetime must be known a priori. Its value is taken as $64.44 \mu \mathrm{s}$, determined from fits to the data. A systematic study regarding this parameter is described in Section 5.5.5. The ratio spectrum is then almost exactly described by just the cosine term,

$$
R(t) \approx A \cos \left(\omega_{a} t\right)
$$

in the absence of other effects in the data.

\subsection{Fitting the data}

The basic five parameter function used to the fit is:

$$
f(t)=\boldsymbol{N}_{\mathbf{0}} \cdot e^{-t / \boldsymbol{\tau}} \cdot\left(1+\boldsymbol{A} \cdot \cos \left(\omega_{a} t+\boldsymbol{\phi}\right)\right),
$$

where the fit parameter for $\omega_{a}$ is recast in terms of a ppm level shift $\boldsymbol{R}$ on a reference frequency,

$$
\omega_{a}=2 \pi \cdot 0.2291 \mathrm{MHz} \cdot\left(1+\boldsymbol{R} \times 10^{-6}\right) .
$$

This reference frequency of $0.2291 \mathrm{MHz}$ is the same reference frequency that was used in E821, and $\boldsymbol{R}$ is blinded at the hardware and software levels 111, 112]. Fitting the data with Equation 5.13, however, is insufficient to properly describe the data. An FFT of the residuals between fit and data is shown in Figure $5 \cdot 15$. There are peaks in the FFT at beam dynamics frequencies corresponding to the $\mathrm{CBO}, \mathrm{VW}$, and some beat frequencies with $\omega_{a}$. In order to properly account for these effects, additional terms need to be added to the fit function.

\footnotetext{
${ }^{10}$ In the current section the actual fit parameters are in bold.
} 


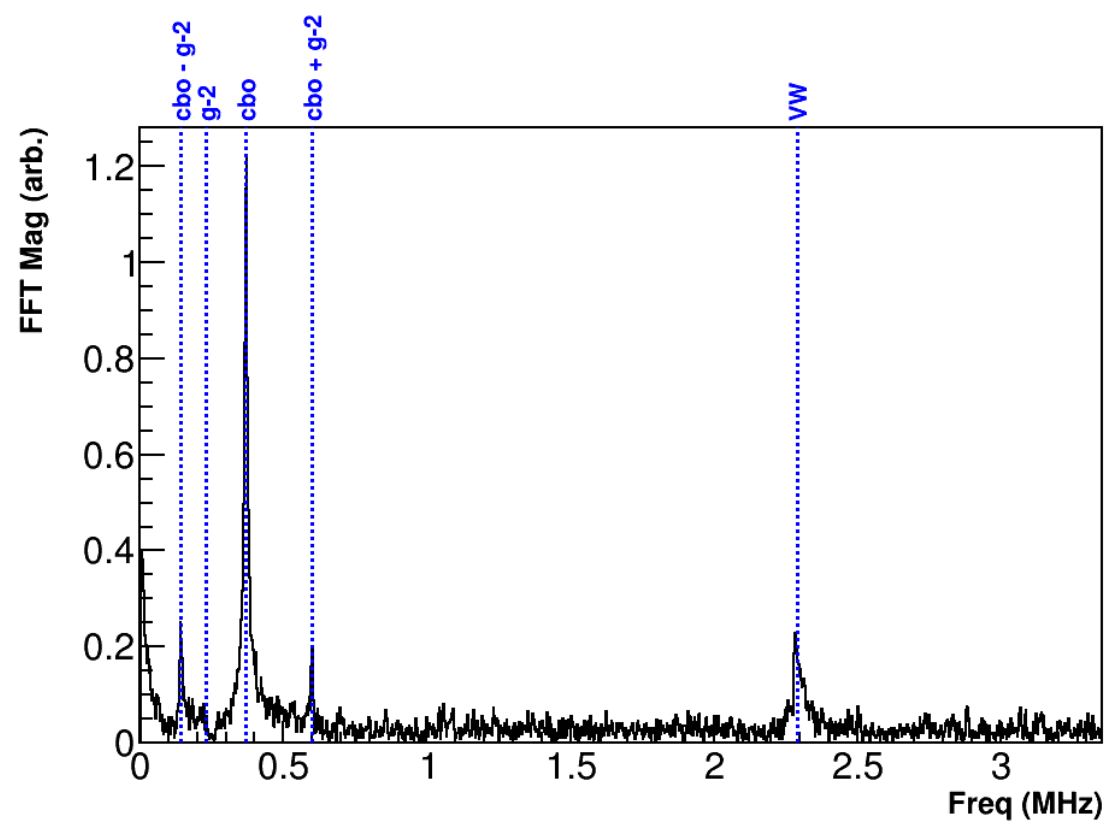

Figure 5·15: FFT of five parameter fit residuals from the $60 \mathrm{~h}$ dataset with no time randomization applied. Peaks corresponding to beam dynamics frequencies of the CBO and VW as well as some beat frequencies with $\omega_{a}$ are readily apparent. A rise at low frequencies corresponds to the effects of the lost muons in the data. The VW peak diminishes with application of the cyclotron period time randomization and disappears entirely with the VW period time randomization.

Equation 5.13 can be expanded to

$$
\begin{array}{r}
f(t)=\Lambda(t) \cdot V(t) \cdot N_{c b o}(t) \cdot N_{2 c b o}(t) \cdot \boldsymbol{N}_{\mathbf{0}} \cdot e^{-t / \boldsymbol{\tau}} \\
\cdot\left(1+A_{c b o}(t) \cdot \cos \left(\omega_{a} t+\phi_{c b o}(t)\right)\right),
\end{array}
$$

where various additional terms have been added in order to account for effects in the data. These terms are described in the following sections. Fitting the data with this function, referred to as the "Threshold Method" or just "T-Method," while not the subject of this dissertation, was done in this analysis as a diagnostic and informative tool for the Ratio Method analysis.

The ratio time spectrum is fit with a function constructed from its definition in 
Equations 5.7 and 5.8, as opposed to an expansion of the three parameter function in Equation 5.12, Including the additional effects previously mentioned, the fit function becomes

$$
\begin{gathered}
R(t)=\frac{2 f(t)-f_{+}(t)-f_{-}(t)}{2 f(t)+f_{+}(t)+f_{-}(t)} \\
f_{ \pm}(t)=f\left(t \pm T_{a} / 2\right), \\
f(t)=\Lambda(t) \cdot V(t) \cdot N_{c b o}(t) \cdot N_{2 c b o}(t) \cdot\left(1+A_{c b o}(t) \cdot \cos \left(\omega_{a} t+\phi_{c b o}(t)\right)\right) .
\end{gathered}
$$

The $f(t)$ given here differs from that in Equation 5.15 in that the $\boldsymbol{N}_{\mathbf{0}} \cdot e^{-t / \boldsymbol{\tau}}$ terms have divided out. Using this function, as opposed to an expansion of the three parameter ratio function, eliminates any approximations made in that three parameter function derivation. Any fit parameters should be consistent in value between the T-Method and Ratio Method results, barring adjustments due to the application of the Ratio Method.

Because the Ratio Method reduces the sensitivity of the $\omega_{a}$ determination to various effects in the data, peaks that appear in the FFT of the five parameter fit residuals do not necessarily appear in the FFT of the three parameter ratio fit residuals; see Figure 5·16. Indeed unless one looks at the FFT over the early part of the fit (eg. first $30 \mu \mathrm{s}$ ) or at the shape of the Ratio Method denominator, one might not know the effects even exist in the data at all, barring occasionally poor $\chi^{2}$ 's. Nevertheless, those effects typically still need to be included in the fit function for a proper evaluation of $\omega_{a}$. Because of the reduction in sensitivity in the ratio fits, there are some parameters which the Ratio Method has trouble fitting by itself. Using the results from a T-Method fit to the data can then be a useful tool for constraining those specific parameters. 


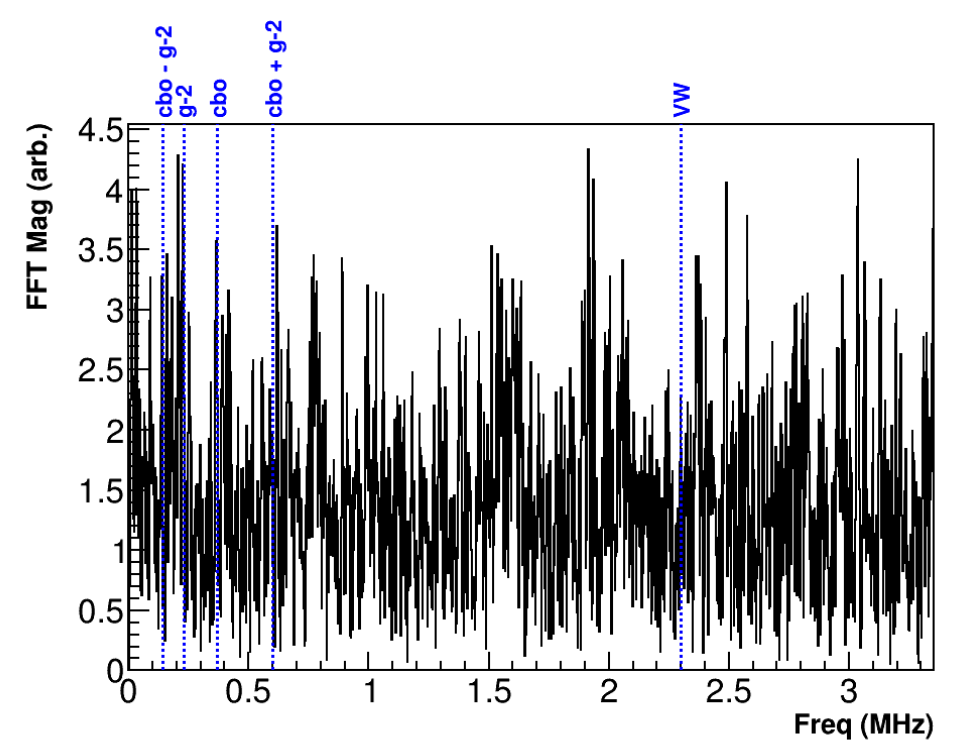

(a) FFT of fit residuals over all times within the fit range. There is no evidence of residual oscillations.

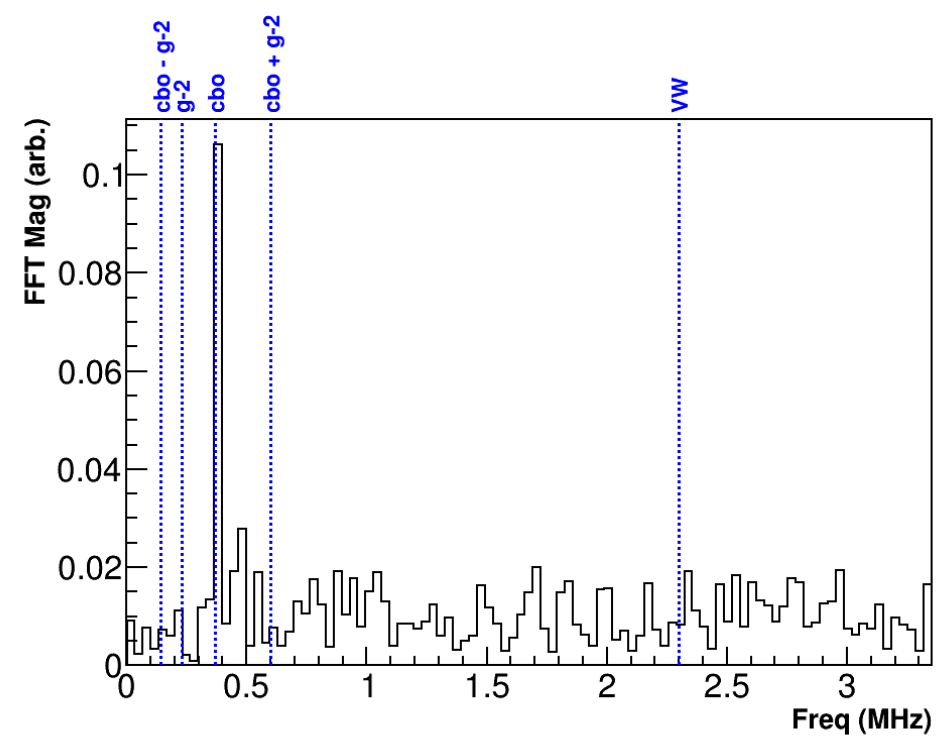

(b) FFT of fit residuals over the first $30 \mu \mathrm{s}$. The CBO peak can be seen above the noise, though the corresponding beat frequencies do not appear.

Figure 5.16: FFT of three parameter ratio fit residuals for the $60 \mathrm{~h}$ dataset. Dashed blue lines indicate various beam dynamics frequencies and their beat frequencies with $\omega_{a}$. 


\subsubsection{CBO terms}

As described in Section 2.6.1, the CBO effect modulates the $\omega_{a}$ oscillation. This shows up as a modification on the five parameter function parameters $\left\{N_{0}, A, \phi\right\} \rightarrow$ $\left\{N_{0} \cdot N_{c b o}(t), A_{c b o}(t), \phi_{c b o}(t)\right\}$ where these terms are given to first order as

$$
\begin{aligned}
& N_{c b o}(t)=\left(1+\boldsymbol{A}_{\boldsymbol{c b o}-\boldsymbol{N}} \cdot e^{-t / \boldsymbol{\tau}_{c b o}} \cdot \cos \left(\omega_{c b o}(t) \cdot t+\boldsymbol{\phi}_{\boldsymbol{c b o}-\boldsymbol{N}}\right)\right), \\
& A_{c b o}(t)=\boldsymbol{A} \cdot\left(1+\boldsymbol{A}_{\boldsymbol{c b o}-\boldsymbol{A}} \cdot e^{-t / \boldsymbol{\tau}_{c b o}} \cdot \cos \left(\omega_{c b o}(t) \cdot t+\boldsymbol{\phi}_{\boldsymbol{c b o}-\boldsymbol{A}}\right)\right), \\
& \phi_{c b o}(t)=\boldsymbol{\phi}_{\mathbf{0}}+\boldsymbol{A}_{\boldsymbol{c b o}-\boldsymbol{\phi}} \cdot e^{-t / \boldsymbol{\tau}_{\boldsymbol{c b o}}} \cdot \cos \left(\omega_{c b o}(t) \cdot t+\boldsymbol{\phi}_{\boldsymbol{c b o}-\boldsymbol{\phi}}\right) .
\end{aligned}
$$

In Equation 5.19 the $\boldsymbol{N}_{\mathbf{0}}$ term is left out since the ratio fit includes the $N_{c b o}(t)$ term but not $\boldsymbol{N}_{\mathbf{0}}$, and in Equation 5.21 , $\phi_{c b o}(t)$ has an additive phase instead of a multiplicative one since $\phi_{0}$ is not an amplitude and can be equal to zero. Each of the terms then includes additional fit parameters in an extra amplitude and phase, as well as one shared CBO lifetime and frequency. As described in Section 4.4, the default model for the $\mathrm{CBO}$ modulation is assumed to be an exponentially decaying envelope, and the CBO frequency, $\omega_{c b o}(t)$, was time-dependent for Run 1. The function for the CBO frequency shown in Figure $4 \cdot 28$ is taken in the fit function as

$$
\omega_{c b o}(t)=\omega_{c b o} \cdot\left(1+\frac{A e^{\left(-t / \tau_{A}\right)}}{\omega_{0} t}+\frac{B e^{\left(-t / \tau_{B}\right)}}{\omega_{0} t}\right),
$$

where $\boldsymbol{\omega}_{\boldsymbol{c} \boldsymbol{b o}}$ is the free fit parameter, and the model parameters $\left\{\omega_{0}, A, \tau_{A}, B, \tau_{B}\right\}$ are fixed from the tracking analysis. These parameters for the various datasets and two tracker stations are given in Table 5.2 .

It should be noted that Equations 5.20 and 5.21 are not necessarily needed in order to get good fits to the data, whereas Equation 5.19 always is. This is typically dataset or random seed dependent. While some datasets had certain parameters with large errors relative to their amplitudes, for this analysis all terms were included in 


\begin{tabular}{lcccccc}
\hline \multicolumn{7}{c}{ CBO Frequency Model Parameters } \\
\hline \hline Dataset & Tracker Station & $\omega_{0}(\mathrm{rad} / \mathrm{\mu s})$ & $A(\mathrm{rad})$ & $\tau_{A}(\mu \mathrm{s})$ & $B(\mathrm{rad})$ & $\tau_{B}(\mathrm{\mu s})$ \\
\hline \multirow{2}{*}{$60 \mathrm{~h}$} & 12 & 2.3389 & 2.9 & 81.8 & 5.12 & 7.7 \\
& 18 & 2.3387 & 2.82 & 81.1 & 5.08 & 8.2 \\
\hline \multirow{2}{*}{ HighKick } & 12 & 2.6145 & 3.27 & 52.8 & 6.96 & 6.6 \\
& 18 & 2.6137 & 3.23 & 46.2 & 6.61 & 6.8 \\
\hline \multirow{2}{*}{ 9d } & 12 & 2.6106 & 2.86 & 72.8 & 5.50 & 8.5 \\
& 18 & 2.6110 & 2.89 & 79.2 & 5.44 & 9.2 \\
\hline \multirow{2}{*}{ Endgame } & 12 & 2.3377 & 7.43 & 95.1 & 4.71 & 9.0 \\
& 18 & 2.3379 & 7.44 & 95.2 & 4.90 & 9.2 \\
\hline
\end{tabular}

Table 5.2: Fixed parameters in the CBO frequency model [113, 114]. The larger values of the $A$ and $\tau_{A}$ parameters for the Endgame dataset are a consequence of the degradation of the quadrupole resistors over the course of Run 1.

all fits. Fits converged properly with appropriate tuning of the starting parameters, justifying their inclusion. When considering higher order $\mathrm{CBO}$ modifications to the fit function, the only term that was found to be necessary was the second order $2 \omega_{c b o}$ modulation on the $\boldsymbol{N}_{\mathbf{0}}$ term, $N_{0} \cdot N_{c b o}(t) \rightarrow N_{0} \cdot N_{c b o}(t) \cdot N_{2 c b o}(t)$, where

$$
N_{2 c b o}(t)=\left(1+\boldsymbol{A}_{2 \boldsymbol{c} \boldsymbol{b} \boldsymbol{o}-\boldsymbol{N}} \cdot e^{-2 t / \tau_{c b o}} \cdot \cos \left(2 \omega_{c b o}(t) \cdot t+\boldsymbol{\phi}_{2 \boldsymbol{c} \boldsymbol{b} \boldsymbol{o}-\boldsymbol{N}}\right)\right) .
$$

The form is assumed to be the same as the first order CBO terms, except the lifetime of the effect is half the CBO lifetime, $\boldsymbol{\tau}_{\boldsymbol{c} b \boldsymbol{o}} / 2$. This is due to the fact that $N_{2 c b o}(t)$ comes from the width of the oscillating beam, as opposed to the oscillating mean. Indeed as will be shown in Section 5.4.1, the inclusion of this term is necessary to get good fits to the per calorimeter data, where the $\mathrm{CBO}$ effect is stronger than in the sum of the data from all calorimeters.

Systematic studies relating to the choice of CBO envelope and choice of fixed parameters in the $\mathrm{CBO}$ frequency model are explored in Section 5.5.3. For runs after Run 1, it may be necessary to include the higher order modifications to the $A_{c b o}(t)$ 


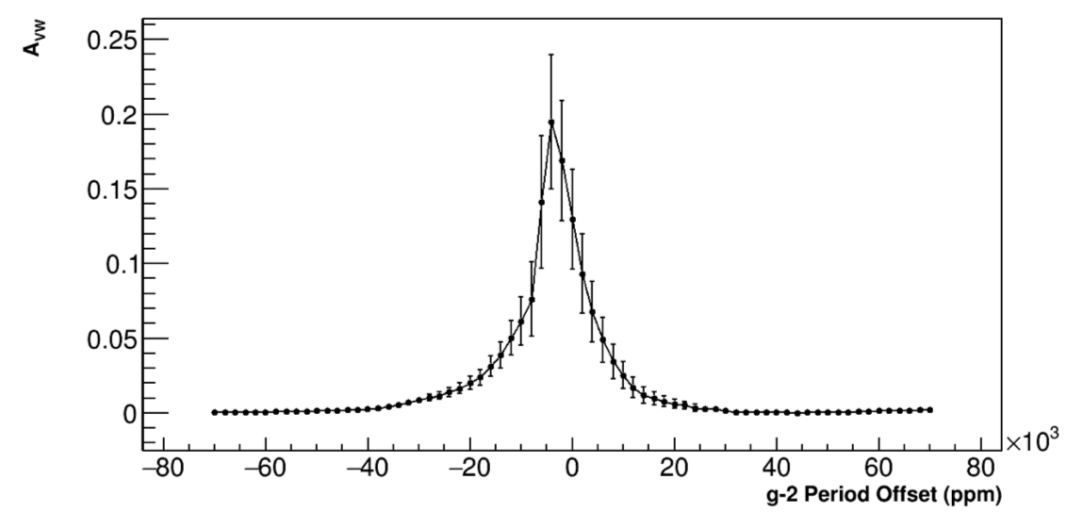

Figure 5·17: Fitted VW amplitude as a function of the choice of offset from $T_{a}$ in units of thousands of ppm for the $60 \mathrm{~h}$ dataset. The default time-shift lies at 0 on this plot, right on the resonance where the VW amplitude blows up. Only by time-shifting by a drastically different amount (which negatively affects $\mathrm{R}$ ), can the resonance be avoided.

and $\phi_{c b o}(t)$ terms.

\subsubsection{VW term}

As mentioned in Section 5.2, the VW effect is time-randomized out of the data, such that

$$
V(t)=1
$$

This is done due to complications observed in the application of the Ratio Method. In the $60 \mathrm{~h}$ and Endgame datasets, the VW frequency was found to be nearly $10 \cdot \omega_{a}$, on a potential resonance. To first order this even multiple frequency implies the VW effect should completely cancel out in the Ratio Method, as shown in Figure 5·14. However, in combination with the fast rotation effect, this leads to a modified envelope for the VW signal in the Ratio Method data and inflated VW amplitudes in the fits [115. See Figures $5 \cdot 17$ and $5 \cdot 18$.

For the 9d dataset, which has a VW frequency that's nearly $9 \cdot \omega_{a}$ and avoids said resonance (and by extension the HighKick), the Ratio Method flattened out the VW 


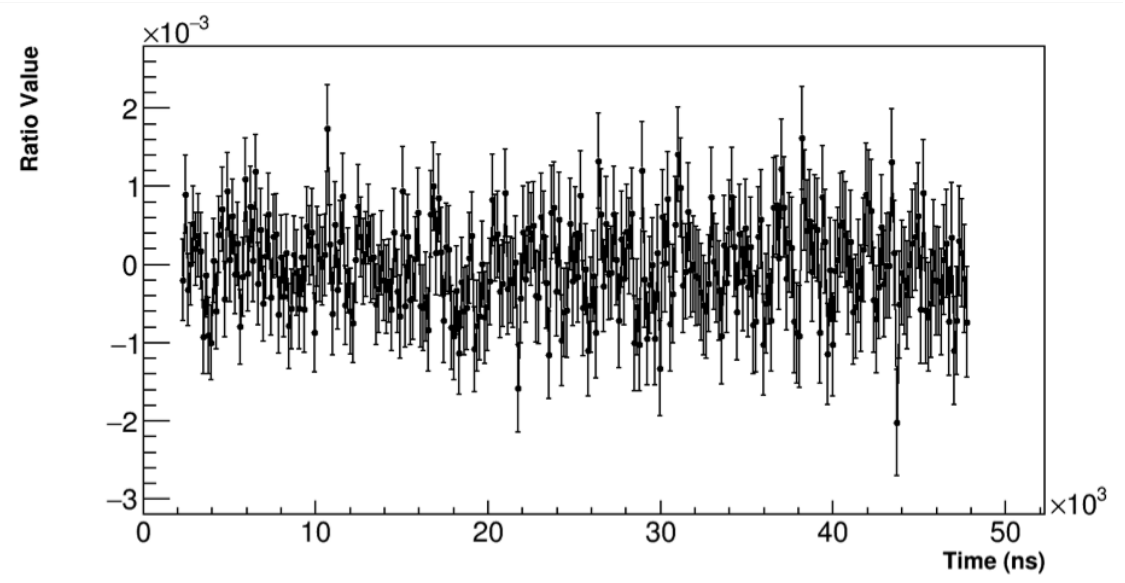

(a) Without the FR effect included.

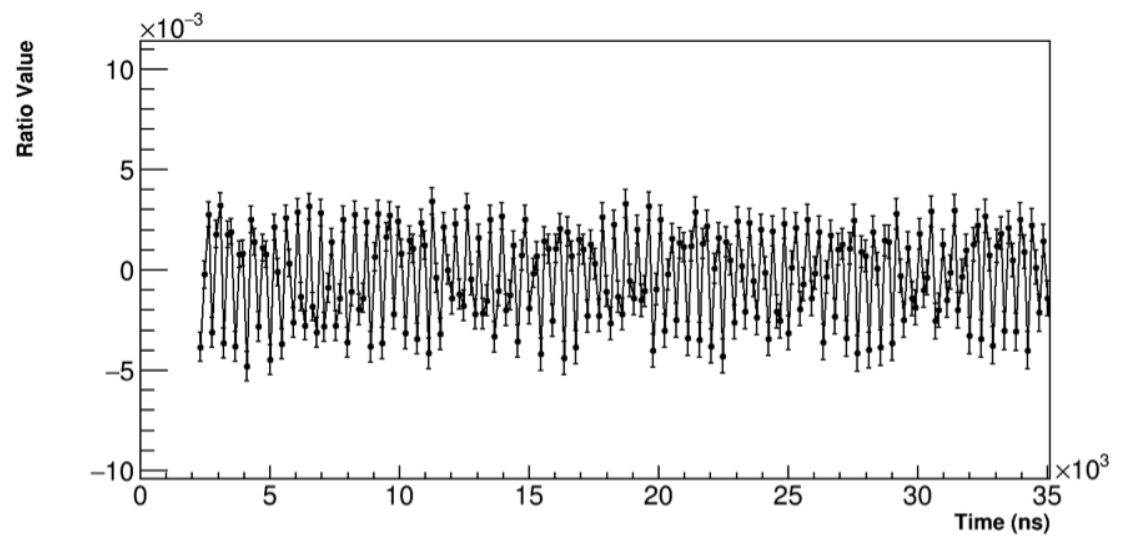

(b) With the FR effect included.

Figure 5.18: Ratio data with (top) and without (bottom) the FR effect from a Toy MC simulation, with a VW effect with a frequency $\omega_{V W}=$ $10 \cdot \omega_{a}$. The $\omega_{a}$ wiggle itself has been removed, and the lifetime of the VW was set to a large number. The top plot shows ratio data which is consistent with 0 after all effects have been removed and the VW has divided out. The bottom plot shows ratio data inconsistent with 0 , with oscillations at the VW frequency, and an interesting beating structure. Note the different scales. 


\section{Change in Asymmetry due to VW Randomization}

\begin{tabular}{lcccc}
\hline \hline Dataset & $A$ no randomization & $A$ with randomization & $\Delta A$ & $\Delta \sigma_{R}(\mathrm{ppb})$ \\
\hline $60 \mathrm{~h}$ & 0.3697 & 0.3637 & -0.0060 & 22.7 \\
HighKick & 0.3707 & 0.3632 & -0.0075 & 29.3 \\
$9 \mathrm{~d}$ & 0.3714 & 0.3639 & -0.0075 & 18.1 \\
Endgame & 0.3747 & 0.3686 & -0.0061 & 10.7 \\
\hline
\end{tabular}

Table 5.3: Asymmetry values in the the Run 1 datasets with and without the VW randomization, and the corresponding change in the statistical error on R. An energy cut of $1700 \mathrm{MeV}$ was applied to the data.

amplitudes as a function of calorimeter number. This lead to a systematically smaller VW amplitude in the calorimeter sum fit compared to that in a T-Method fit. The simplest means to remove both of these problems was to randomize out the VW effect entirely. Table 5.3 gives the change in asymmetry and corresponding change in the statistical error on $\mathrm{R}$ due to this additional time randomization for the Run 1 datasets. The additional randomization increased the statistical error on $\mathrm{R}$ by a small amount, and changed the mean value of $\mathrm{R}$ for many random seed fits to the data slightly ${ }^{11}$. In the analysis of future runs, it may be necessary to include the proper VW envelope in the fits using a functional form of the FR instead of randomizing out the effect.

The function form for the VW as it is used in the T-Method fits is taken identically to the CBO terms,

$$
V(t)=1+\boldsymbol{A}_{\boldsymbol{V} \boldsymbol{W}} \cdot e^{-t / \boldsymbol{\tau}_{\boldsymbol{V} W}} \cos \left(\omega_{V W}(t) \cdot t+\boldsymbol{\phi}_{\boldsymbol{V} \boldsymbol{W}}\right),
$$

with an exponentially decaying envelope, and additional amplitude and phase parameters. The VW frequency, given in Equation 2.26, is dependent on the cyclotron frequency and vertical betatron frequency. Using Equations 2.20 and 2.25, the VW

\footnotetext{
${ }^{11}$ This new mean value is statistically consistent with the results without the additional randomization 115.
} 
dependence on the CBO frequency is determined as

$$
\begin{aligned}
\omega_{V W}(t) & =2 \pi\left(f_{c}-2 f_{y_{B O}}\right), \\
& =2 \pi\left(f_{c}-2 f_{c b o}(t) \sqrt{2 f_{c} / f_{c b o}(t)-1}\right),
\end{aligned}
$$

where $f_{c b o}(t)=\omega_{c b o}(t) / 2 \pi$ is determined in the tracking analysis as given by Equation 5.22 . While Equation 5.26 is the theoretical frequency for the VW effect in the continuous quadrupole approximation, it was found in the tracking analysis that including an adjustment factor on the CBO frequency $f_{c b o} \rightarrow \kappa f_{c b o}$ resulted in better

agreement with the directly measured VW frequency [103, 116. In the fit function, the VW frequency is then taken as

$$
\omega_{V W}(t)=2 \pi\left(f_{c}-2 \cdot \boldsymbol{\kappa}_{\boldsymbol{V} \boldsymbol{W}} \cdot f_{c b o}(t) \sqrt{2 f_{c} /\left(\boldsymbol{\kappa}_{\boldsymbol{V} \boldsymbol{W}} \cdot f_{c b o}(t)\right)-1}\right)
$$

where now the VW frequency fit parameter is $\boldsymbol{\kappa}_{\boldsymbol{V} \boldsymbol{W}}$. In fits to the data, this adjustment factor ends up being on the order of about a percent.

\subsubsection{Lost muons}

Muons lost from the storage ring during the frequency analysis portion of each fill will distort the observed decay positron spectrum. These hits show up as a rise at low frequencies in the FFT of the fit residuals due to the slow nature of the effect; see Figure 5.15. These muon losses typically originate from those muons with large betatron amplitudes which hit material near the edge of the storage ring, or those muons which experience local field perturbations one too many times. In both cases the muons will lose energy and spiral inward out of the ring, some of which will then pass through multiple calorimeters. Because lost muons are MIPs, they are relatively easily identified by their small energy deposition in hit calorimeters, approximately $170 \mathrm{MeV}$. These lost muons typically have a flight time between adjacent calorimeters 

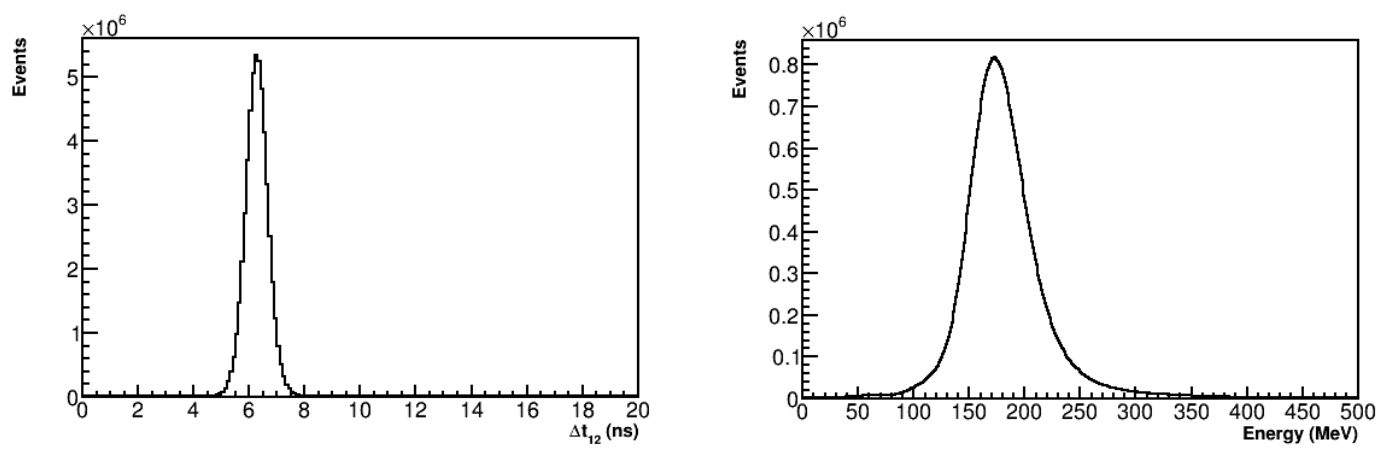

Figure 5·19: Time-of-flight $\Delta t$ (left) and energy deposition (right) distributions for lost muons passing through adjacent calorimeters, where counts have been included from hits in both calorimeters. Typical flight times and energy depositions are $6.25 \mathrm{~ns}$ and $170 \mathrm{MeV}$ respectively. Data are from the Endgame dataset.

of $\Delta t_{12}=6.25 \mathrm{~ns}$ [106, 117]. The $\Delta t$ and energy deposition distributions are shown in Figure 5.19. By looking for coincidences between three adjacent calorimeters, or triples, and then applying cuts and subtracting backgrounds, a pure spectrum of lost muons can be constructed ${ }^{12}$. This lost muon spectrum $L(t)$ can then be implemented into the fit function in order to account for the positrons that would have been observed later in the fill in the absence of losses.

The cuts used for the lost muon selection are given in Table 5.4. Triple coincidences are only included where every cluster consists of three or fewer crystals hit, with $80 \%$ of the energy deposited in one crystal, indicative of MIP events. $\Delta t$ and energy deposition ranges are taken as $5 \mathrm{~ns} \leq \Delta t_{12,23} \leq 7.5 \mathrm{~ns}$ and $100 \mathrm{MeV} \leq E_{1,2,3} \leq$ $250 \mathrm{MeV}$ respectively, where the ranges come from inspection of Figure $5 \cdot 19$. The $\Delta t_{12}$ distribution as a function of time in-fill is shown in Figure $5 \cdot 20$. By examining this distribution in the range $2 \mathrm{~ns} \leq \Delta t_{12} \leq 4 \mathrm{~ns}$, and averaging the contained counts, an approximation for the accidental background can be determined and subtracted from the triples spectrum. The accidental background typically comes from either

\footnotetext{
${ }^{12}$ Using triples rather than doubles helps reduce the accidental background while keeping enough statistics.
} 
double coincidences and a real positron hit, or a particle shower induced by an incident positron which hits an adjacent calorimeter.

\begin{tabular}{lc}
\hline \multicolumn{2}{c}{ Lost Muon Cuts } \\
\hline \hline Parameter & Cut \\
\hline Cluster size & $\leq 3$ crystals \\
Cluster energy fraction & $\geq 0.8$ in main crystal \\
Time of flight between adjacent calorimeters & $5 \mathrm{~ns} \leq \Delta t_{12,23} \leq 7.5 \mathrm{~ns}$ \\
Energy deposition & $100 \mathrm{MeV} \leq E_{1,2,3} \leq 250 \mathrm{MeV}$ \\
Time of flight between separated calorimeters & $\Delta t_{13} \leq 14.4 \mathrm{~ns}$ \\
\hline
\end{tabular}

Table 5.4: Lost muon selection cuts. In a triple coincidence the subscripts 1, 2, and 3 correspond to the three calorimeters hit clockwise around the ring.

Also shown in Figure $5 \cdot 20$ are two bands of stable beam contaminants corresponding to stored deuterons and protons. These particles have different time-of-flights between calorimeters due to their larger masses. By looking at the $\Delta t_{13}$ distribution for times greater than $300 \mu \mathrm{s}$, so that most muons have already decayed, the deuteron population is easily isolated; see Figure $5 \cdot 21$. While the deuteron population is mostly removed by the $\Delta t_{12}$ cut, an additional cut of $\Delta t_{13} \leq 14.4 \mathrm{~ns}$ helps remove any remaining deuteron contamination. The proton population, due to its similarity to the real lost muon population, is harder to remove. The simplest solution is to simply cut on the negative side of the $\Delta t_{12}$ or $\Delta t_{13}$ distributions. See Section 5.5.4 for the results using this additional cut. It was found that the proton contamination makes almost no difference to the fitted value of $\mathrm{R}$. The default choice then is to use the previously specified cut ranges which increase the amount of statistics in the lost muons distribution, which in some cases improves fit convergence. A study of the exact rate of these beam contaminants is included in Reference [118].

The last significant background is the quadruples spectrum. Due to how the triple coincidences are constructed, real quadruples will be counted as two separate triples. 


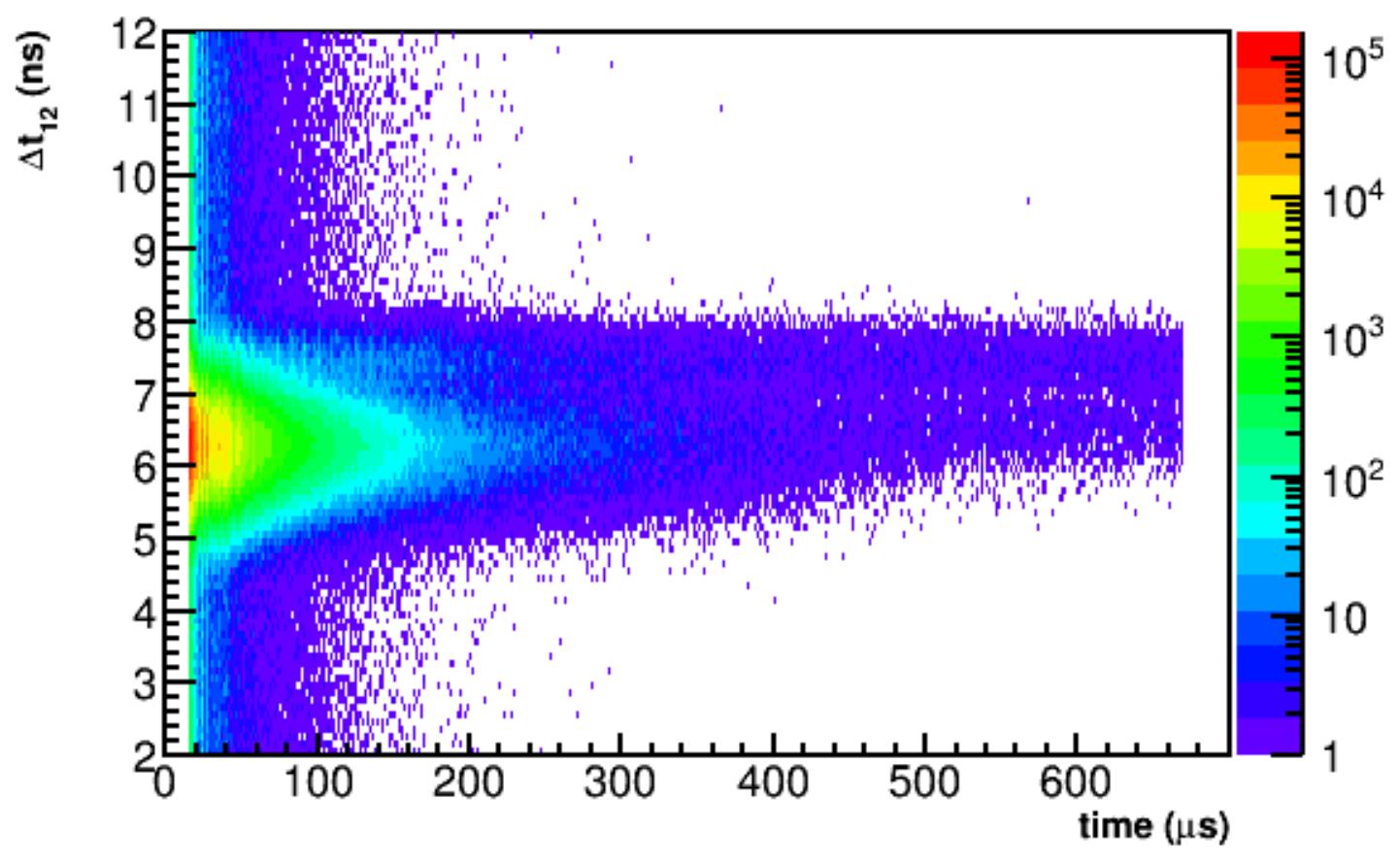

Figure 5.20: $\Delta t_{12}$ distribution as a function of time in-fill before any cuts. Note the log color scale. Lost muons have a $\Delta t_{12}$ distribution centered at $6.25 \mathrm{~ns}$. The accidental background can be seen as counts out at $\Delta t$ 's far from the center of the distribution. Color striations in the core of the distribution correspond to CBO periods. There are two bands of hits that do not fall off as severely with time as the lost muons do. The band contained mostly between 7 and 8 ns corresponds to deuterons, while the band contained mostly between 6 and 7 ns corresponds to protons. Data are from the Endgame dataset. 


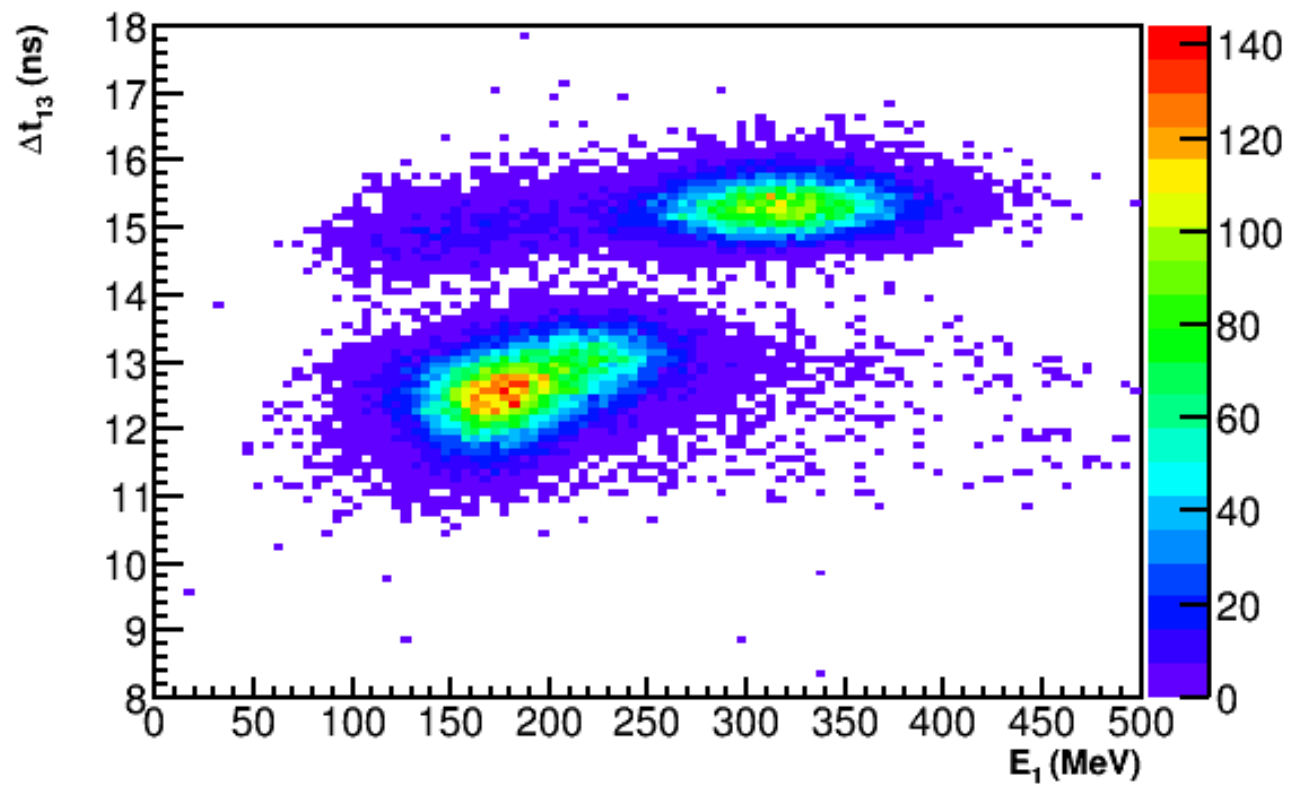

Figure 5.21: $\Delta t_{13}$ distribution as a function of energy for times greater than $300 \mathrm{\mu s}$. The lost muons are centered at $170 \mathrm{MeV}$ and $\Delta t_{13} \approx$ $12.5 \mathrm{~ns}$, while the deuterons are at $\Delta t_{13} \geq 14.4 \mathrm{~ns}$. While the deuterons have a preferentially larger energy deposition, they can be seen to extend to low energies, making cutting on energy unrealistic. Though not easily separated by eye, the stored protons are contained within the upper right portion of the lost muons. Data are from the Endgame dataset.

While the quadruples spectrum produces a purer sample of lost muons, the statistics are much reduced, and similarly for higher order coincidences. The quadruples spectrum is constructed in the same was as the triples with the same cut ranges. The quadruple background is removed by subtracting off both triples which originated from quadruple coincidences.

Figure $5 \cdot 22$ shows the final $\Delta t_{12}$ distribution as a function of time in-fill for selected lost muons with cuts. The final triple losses spectrum $L(t)$ is shown in Figure $5 \cdot 23$, in comparison to both the quadruples and accidentals spectra. Once the lost muon spectrum has been constructed, it needs to be included in the fit function to account for the changing number of hits over the course of the fill. The true lost muon rate 


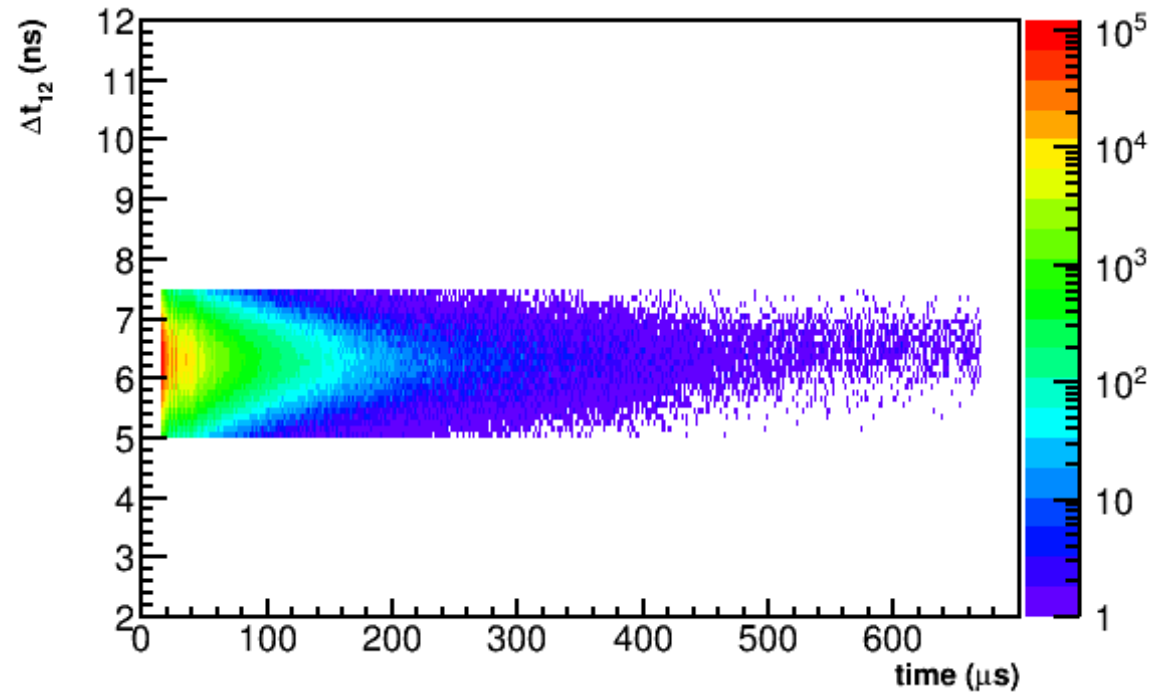

Figure 5.22: Final $\Delta t_{12}$ distribution for selected lost muons as a function of time in-fill. Data are from the Endgame dataset.

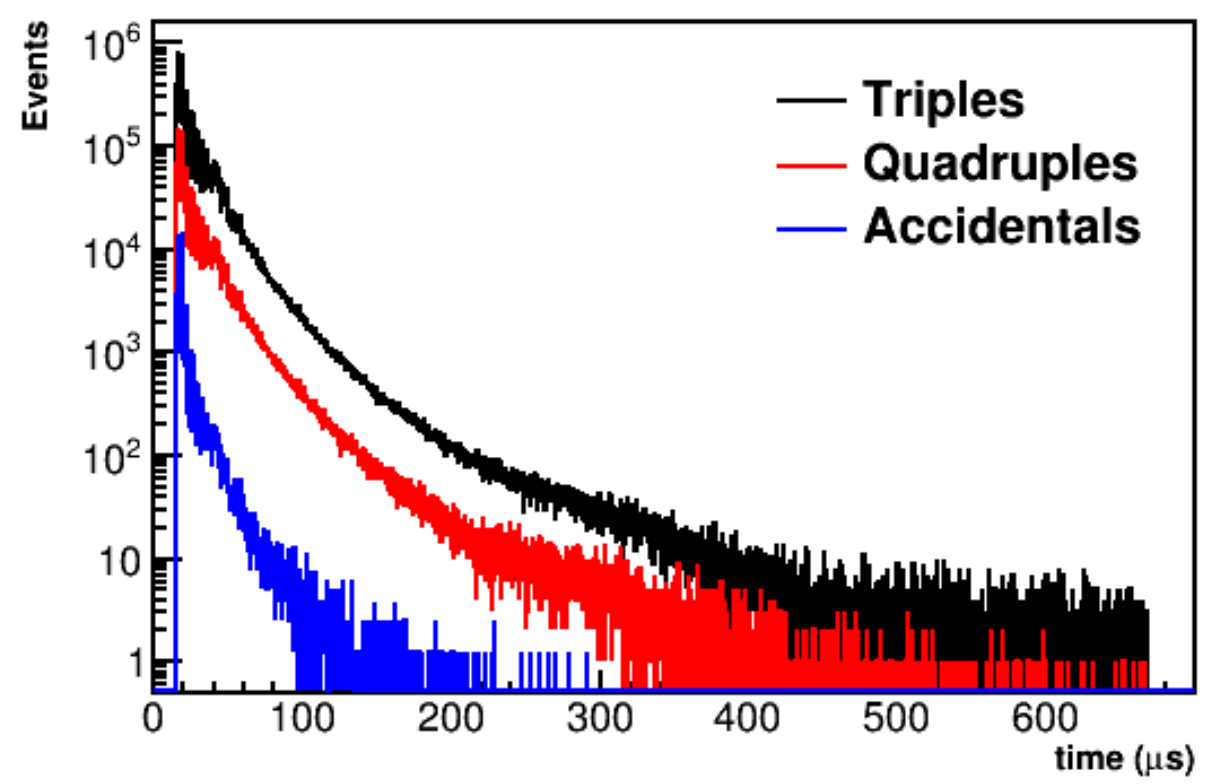

Figure 5.23: Lost muon triples spectrum $L(t)$ as a function of time in-fill, after all cuts and background subtractions. Also shown are the quadruples and accidental background spectra. The shape of the triples and quadruples spectra are nearly the same. The shape of the triples spectrum comes from beam dynamics (CBO) and lost muon acceptance effects. Data are from the Endgame dataset. 
will be given by $L(t) / \epsilon$, where $\epsilon$ is the loss detection efficiency. The change in the number of muons within the storage ring can be written as

$$
d N=-\frac{N}{\tau} d t-\frac{L(t)}{\epsilon} d t
$$

with the solution

$$
N(t)=N_{0} \cdot e^{-t / \tau} \cdot\left(1-\frac{1}{\epsilon N_{0}} \int_{t_{0}}^{t} L\left(t^{\prime}\right) e^{\left(t^{\prime} / \tau\right)} d t^{\prime}\right) .
$$

The parameter $\tau$ is the same muon lifetime as in the T-Method fit function, except here it is set at the default value of $64.44 \mu s^{13}$. The value for $t_{0}$ can be taken as any time at or before the start of the fitting range, as it simply changes the scale of $N_{0}$. The modification $\Lambda(t)$ as listed in Equation 5.15 is then

$$
\Lambda(t)=1-\kappa_{\text {loss }} \int_{t_{0}}^{t} L\left(t^{\prime}\right) e^{\left(t^{\prime} / \tau\right)} d t^{\prime}
$$

where $\boldsymbol{\kappa}_{\text {loss }}=1 / \epsilon N_{0}$ is the fit parameter ${ }^{14}$.

It should be noted that the Ratio Method is largely insensitive to the slow lost muons effect, which divides out. No rise at low frequencies appears in the FFT of the ratio fit residuals, Figure 5·16, and when letting $\boldsymbol{\kappa}_{\text {loss }}$ float, the fit does not converge. However, $\boldsymbol{R}$ changes on the order of tens of ppb when the lost muon term is included with $\boldsymbol{\kappa}_{\text {loss }}$ fixed to the value determined from a T-Method fit, so by default it is included. See Section 5.5.4 for more discussion on this.

\footnotetext{
${ }^{13}$ The fitted $\omega_{a}$ frequency is largely insensitive to this parameter, and especially so in the Ratio Method fits, so this is acceptable. If necessary, the fitting procedure can be iterated.

${ }^{14} \mathrm{By}$ construction $\kappa_{\text {loss }}$ is a very small parameter, $\mathcal{O}\left(10^{-10}\right)$. This factor is absorbed into the parameter such that the fit parameter is $\mathcal{O}(1)$.
} 


\subsubsection{Fit procedures and parameters}

$\chi^{2}$ fits are performed on the pileup corrected positron time spectra and ratio data in ROOT using the standard TH1F and TGraph fit methods with a strategy level of two. Fits are performed in stages where groups of associated parameters are freed, fit, and then fixed before fitting the next set of parameters. In the final fit all parameters are free. Each of the four Run 1 precession frequency analysis datasets are fit with 14 parameters. 13 parameters are free in fits to the 60h, 9d, and Endgame datasets, with the $\kappa_{\text {loss }}$ parameter determined and fixed from a T-Method fit. In the HighKick dataset, $\tau_{c b o}$ is also fixed from a T-Method fit, as the lifetime is relatively short and the Ratio Method can't successfully fit it, resulting in 12 free fit parameters. In the T-Method fits there are two extra free fit parameters, those being the $N_{0}$ and $\tau$ terms which are divided out of the Ratio Method fits.

Fits to calorimeter sum spectra are done over the range 30.2-650 $\mu$ s, corresponding to 4155 bins 15 , The choice of fit start time was made to allow for the muon beam to stabilize in the storage region after the scraping procedure. In addition, the start time was chosen to lie directly on a $g-2$ zero crossing, which from E821 experience was shown to reduce some systematic errors.

The fit end time was set so that the random variations in bin contents were comfortably Gaussian. For the same reason, individual calorimeter fits with less data were performed out to 400 us. Table 5.5 gives the various fit procedure parameters.

As a reminder, $R$ is blinded at the hardware and software levels [111, 112]. The software blinding string used for the $60 \mathrm{~h}$ dataset was different than that used when fitting the HighKick, 9d, and Endgame datasets, each of which used the same blinding string. This was done in order to perform a software-level relative un-blinding exercise between different analyzers with the $60 \mathrm{~h}$ dataset in order to determine if there were

\footnotetext{
${ }^{15}$ The fit range is not defined on exact bin edges, and those bins where the fit begins and ends are included in the fit.
} 
Fit Procedure Parameters

\begin{tabular}{lc}
\hline \hline Parameter & Value \\
\hline Fit strategy level & 2 \\
Fit start time & $30.2 \mu \mathrm{s}$ \\
Fit end time (calorimeter sum) & $650 \mu \mathrm{s}$ \\
Fit end time (single calorimeters) & $400 \mu \mathrm{s}$ \\
Bins in fit (calorimeter sum) & 4155 \\
Number of free fit parameters (60h, 9d, Endgame) & 13 \\
Number of free fit parameters (HighKick) & 12 \\
\hline
\end{tabular}

Table 5.5: Various parameters used in the fit procedure.

\begin{tabular}{lr}
\hline & SAM Dataset Names \\
\hline \hline Dataset & Name \\
\hline $60 \mathrm{~h}$ & gm2pro_daq_full_run1_60h_5039A_goldList \\
HighKick & gm2pro_daq_full_run1_HighKick_5040A_goldList \\
$9 \mathrm{~d}$ & gm2pro_daq_full_run1_9d_5039A_goldList \\
Endgame & gm2pro_daq_full_run1_EndGame_5040A_goldList \\
\hline
\end{tabular}

Table 5.6: The SAM dataset names of the Run 1 precession frequency analysis datasets.

any obvious problems in the various analyses [119, 120]. Therefore in the following results, $R$ values between different datasets are comparable between the datasets except for the $60 \mathrm{~h}$, barring any differences due to field conditions, which are not discussed in this dissertation.

The names of the specific SAM (Sequential Access via Metadata) datasets that were analyzed for this dissertation are given in Table 5.6. SAM is a data handling system used at Fermilab to store and retrieve files along with associated metadata. The specific DQC cuts, gain corrections, processed runs, production software versions, etc. can all be recovered by examining the associated SAM metadata. 


\subsection{Fit results}

Figure $5 \cdot 24$ shows fits to the four Run 1 precession frequency analysis datasets for single random seeds. Table 5.7 gives all fit parameters and their errors. In each dataset case the $\chi^{2} / \mathrm{NDF}$ is acceptable as evidenced by the p-value included in the table results. Fit pulls and the FFT of the fit residuals for the $60 \mathrm{~h}$ dataset are provided in Figure 5.25. As shown all structure has been eliminated within the fit residuals implying that all effects in the data have properly been accounted for in the fit function. The same checks were made for the HighKick, 9d, and Endgame datasets and in each case no residual structure remained. Appendix $\mathrm{D}$ provides the correlation matrices for the various datasets. The only fit parameter that is significantly correlated with $R$ is the $g-2$ phase in all datasets. This increases the confidence in the $\omega_{a}$ extraction, as effects in the data which might potentially be mismodeled will only weakly correlate with the final fitted $R$ value. The various different CBO parameters are self-correlated to different degrees depending on the parameter and the dataset that is being fit. Typically either the phases and frequencies are correlated, or the lifetimes and amplitudes.

The $g-2$ phases for the different datasets showed small differences, due primarily to upstream beam adjustments before injection into the storage ring. Similarly, slightly different asymmetries can be attributed to very small acceptance differences in the stored beams. As described in Section 5.3.3, the value for $\kappa_{\text {loss }}$ was determined and fixed from a T-Method fit to the data. The values themselves don't directly correspond to the level of losses, as each dataset has it's own loss function $L(t)$. 

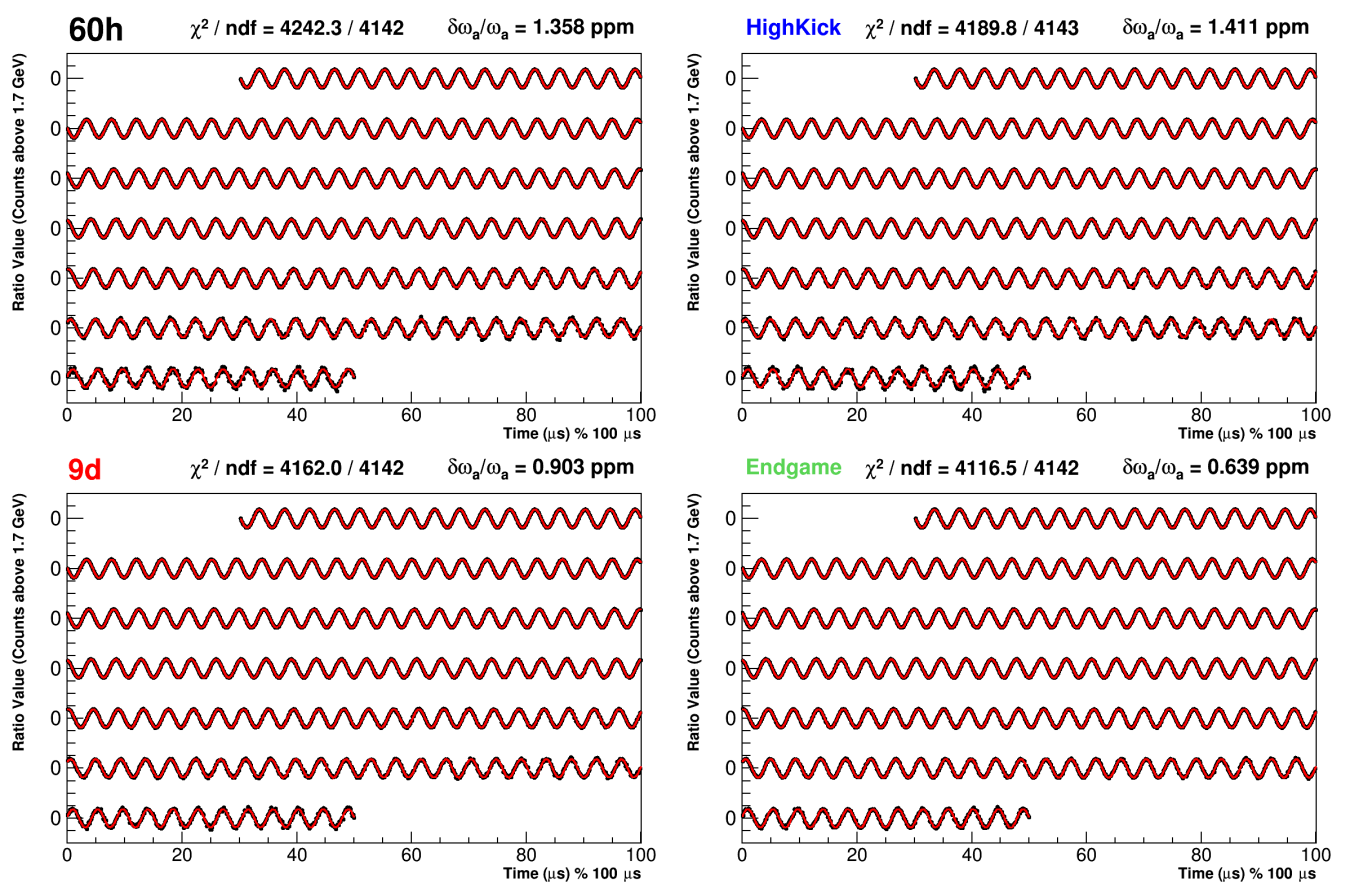

Figure 5.24: Single random seed fits to calorimeter summed data of Run 1 precession frequency analysis datasets. Data are in black and the fits are in red. The X-axis is in units of $\mu$ s modulo $100 \mu \mathrm{s}$, with successive portions of the data points and fit shifted downwards on the plot. The fit range extends from 30.2-650 $\mathrm{s}$. Dataset names are given in the upper left corners of the figures, alongside the $\chi^{2}$ per degree of freedom and relative error on $\omega_{a}$. 


\begin{tabular}{|c|c|c|c|c|c|c|c|c|}
\hline \multicolumn{9}{|c|}{ Ratio Method Fit Results } \\
\hline & \multicolumn{2}{|c|}{$60 \mathrm{~h}$} & \multicolumn{2}{|c|}{ HighKick } & \multicolumn{2}{|c|}{$9 \mathrm{~d}$} & \multicolumn{2}{|c|}{ Endgame } \\
\hline $\begin{array}{l}\chi^{2} / \mathrm{NDF} \\
\mathrm{p} \text {-value }\end{array}$ & \multicolumn{2}{|c|}{$\begin{array}{c}4242 / 4142 \\
0.1356\end{array}$} & \multicolumn{2}{|c|}{$\begin{array}{c}4190 / 4143 \\
0.3018\end{array}$} & \multicolumn{2}{|c|}{$\begin{array}{c}4162 / 4142 \\
0.4104\end{array}$} & \multicolumn{2}{|c|}{$\begin{array}{c}4116 / 4142 \\
0.6079\end{array}$} \\
\hline Parameter & Value & Error & Value & Error & Value & Error & Value & Error \\
\hline $\bar{A}$ & 0.3637 & $4.4 \times 10^{-5}$ & 0.3632 & $4.6 \times 10^{-5}$ & 0.3639 & $2.9 \times 10^{-5}$ & 0.3686 & $2.1 \times 10^{-5}$ \\
\hline$R$ (ppm, blinded) & -20.8479 & 1.3581 & -17.5433 & 1.4112 & -17.8214 & 0.9033 & -17.5674 & 0.6393 \\
\hline$\phi$ & 2.091 & $2.2 \times 10^{-4}$ & 2.081 & $2.3 \times 10^{-4}$ & 2.080 & $1.5 \times 10^{-4}$ & 2.076 & $1.1 \times 10^{-4}$ \\
\hline$\omega_{c b o}(\mathrm{rad} / \mu \mathrm{s})$ & 2.338 & $1.4 \times 10^{-3}$ & 2.599 & $6.6 \times 10^{-3}$ & 2.615 & $5.6 \times 10^{-3}$ & 2.339 & $0.8 \times 10^{-3}$ \\
\hline$\tau_{c b o}(\mu \mathrm{s})$ & 175.2 & 46.8 & 99.4 & 0 & 137.4 & 62.0 & 200.3 & 33.5 \\
\hline$A_{c b o-N}\left(\times 10^{-4}\right)$ & 43.1 & 5.0 & 42.8 & 9.9 & 39.3 & 9.7 & 32.3 & 2.0 \\
\hline$\phi_{c b o-N}$ & -2.343 & 0.107 & 3.817 & 0.446 & 3.302 & 0.374 & -0.710 & 0.062 \\
\hline$A_{2 c b o-N}\left(\times 10^{-4}\right)$ & 1.9 & 1.3 & 4.9 & 4.5 & 2.2 & 2.7 & 1.2 & 0.5 \\
\hline$\phi_{2 c b o-N}$ & 3.331 & 0.638 & 5.665 & 1.274 & -4.936 & 1.127 & 0.322 & 0.448 \\
\hline$A_{c b o-A}\left(\times 10^{-4}\right)$ & 5.5 & 3.9 & 9.5 & 4.1 & 6.4 & 2.5 & 2.7 & 1.9 \\
\hline$\phi_{c b o-A}$ & -0.271 & 0.737 & -2.073 & 0.600 & 1.750 & 0.561 & -2.825 & 0.686 \\
\hline$A_{c b o-\phi}\left(\times 10^{-4}\right)$ & 8.0 & 4.2 & 5.7 & 4.4 & 8.8 & 3.1 & 1.9 & 1.9 \\
\hline$\phi_{c b o-\phi}$ & -1.183 & 0.533 & 1.227 & 0.920 & 4.313 & 0.415 & -1.576 & 0.995 \\
\hline$\kappa_{\text {loss }}$ & 8.974 & 0 & 5.651 & 0 & 2.510 & 0 & 2.345 & 0 \\
\hline
\end{tabular}

Table 5.7: Fit parameters for the four Run 1 precession frequency analysis datasets for a single random seed. The bold row highlights the fitted $R$ values and their respective errors. The $60 \mathrm{~h}$ dataset has a different blinding to the rest. The $\kappa_{\text {loss }}$ parameter is fixed in each fit, with a corresponding error of 0 , and similarly for $\tau_{c b o}$ in the fit to the HighKick dataset. 

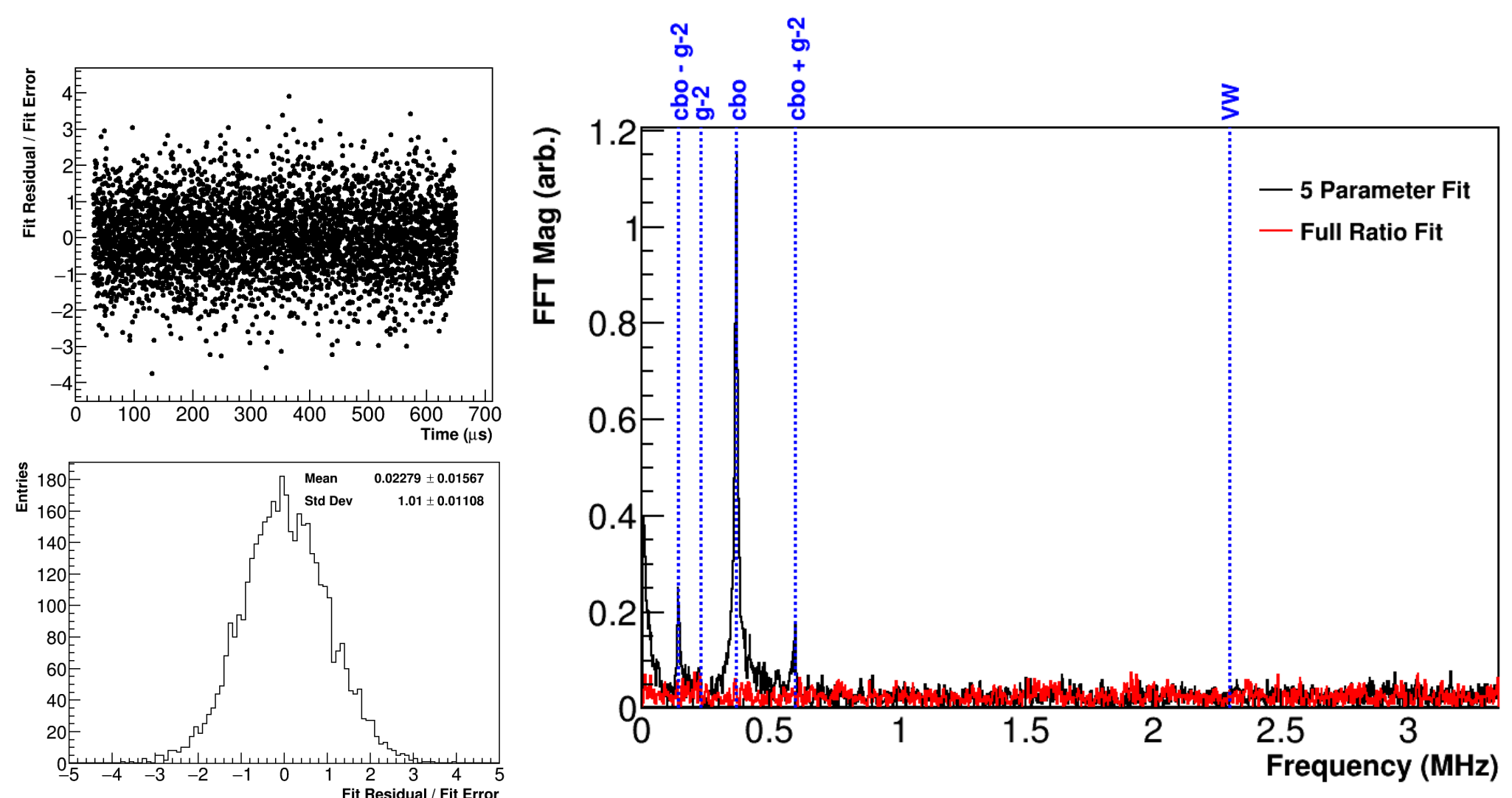

Figure 5·25: Ratio fit pulls (top-left), their projection onto the Y-axis (bottom-left), and the FFTs of fit residuals (right) for a ratio fit (red) and five parameter fit (black) to the 60h dataset. Note the pull projection has a Gaussian shape centered around zero with unit width. In the FFT blue dashed lines are overlaid which correspond to the main beam dynamics peaks which appear in the data. There is no obvious structure in the pulls and no remaining peaks above the noise in the ratio fit FFT. 
The CBO frequencies for the $60 \mathrm{~h}$ and Endgame datasets with $n$ values of 0.108 were found to be 2.338 and $2.339 \mathrm{rad} / \mathrm{\mu s}$ respectively, corresponding to approximately $0.37 \mathrm{MHz}$. For the HighKick and 9d datasets with $n$ values of 0.120 , the CBO frequencies were found to be 2.559 and $2.615 \mathrm{rad} / \mathrm{\mu s}$ respectively, corresponding to approximately $0.415 \mathrm{MHz}$. These frequencies correspond to the expected frequencies as described in Section 2.6, with some slight deviations due to statistics, bad resistors, and the reduced sensitivity in the Ratio Method ${ }^{16}$. The CBO lifetimes between the different datasets are relatively consistent, barring the HighKick dataset for which a smaller CBO lifetime was measured, attributed to the changing behavior of the bad resistors in that dataset. CBO lifetimes in the Ratio Method fits typically converge to values with large errors, compared to in T-Method fits, due to the reduction in sensitivity in the Ratio Method. In the HighKick dataset, the CBO lifetime did not converge properly in the ratio fits, and was therefore fixed to that from a T-Method fit. The main CBO amplitudes, $A_{c b o-N}$, for the various datasets were on the order of $0.3-0.4 \%$, while the higher order CBO amplitudes were in general an order of magnitude less. The strength of the various higher order CBO amplitudes fluctuated among the different datasets. In some cases, the errors on the higher order CBO term amplitudes were comparable to the amplitude itself. While this implies these terms can be dropped from the fit function, all terms were included for analysis uniformity among the different datasets.

The final statistical errors on $R$ for the 60h, HighKick, 9d, and Endgame datasets are $1.358,1.411,0.903$, and $0.639 \mathrm{ppm}$ respectively. The single seed $R$ results for the HighKick, 9d, and Endgame datasets, all of which used the same blinding string, are all well within $1 \sigma$ of each other. The average $R$ value for fits to 50 different random seeds are provided in Section 5.4.4.

\footnotetext{
${ }^{16}$ The VW frequencies, though time-randomized out in the analysis presented here, were found to be approximately 2.30 and $2.04 \mathrm{MHz}$ for the datasets with $n=0.108$ and $n=0.120$ respectively.
} 
Beyond looking at single fit residuals to evaluate the integrity of the fits, other checks were made to verify consistency. In general these checks consisted of slicing up the data in different ways and fitting the subsets. These tests and scans included fitting individual calorimeters, modifying the fit start and end times, adjusting the applied energy thresholds, and fitting individual beam bunches.

\subsubsection{Individual calorimeter fits}

Fits to all 24 time spectra from the individual calorimeters for each of the datasets were performed with the same number of free fit parameters as used in the calorimeter sum fits. Figure $5 \cdot 26$ shows the $\chi^{2} / \mathrm{NDF}^{\prime}$ s for the calorimeter fits; as shown they are evenly spread around 1. Figure $5 \cdot 27$ shows the fitted $R$ values as a function of calorimeter number. Straight line fits were performed to the $R$ values, where in each dataset case the fit returned a good $\chi^{2}$ and a fitted constant that was consistent with the calorimeter sum fit $R$ values. Examining the $R$ values as a function of calorimeter between datasets, particular calorimeter numbers do not tend to lie above or below the fitted average in any systematic way. The spread in $R$ values for each calorimeter then can be said to be driven statistically, though it should be noted that with the larger error bars on the individual calorimeter fits it is hard to tell if there are any systematic shifts one way or another.

Figures $5 \cdot 28$ and $5 \cdot 29$ show calorimeter fit results for the other free parameters for the Endgame dataset. The $g-2$ phases are distinct among the different calorimeters, which can be attributed to different acceptances from the different levels of material upstream. This is particularly noticeable in calorimeters 13 and 19 which sit behind the tracker stations, and have correspondingly smaller phases. Any correlated effects on $R$ are not immediately observed, and might potentially be hidden behind the large errors of the fit. Similarly, the different calorimeters have different fit asymmetries,

once again due to their different acceptances. The CBO parameters are in general 

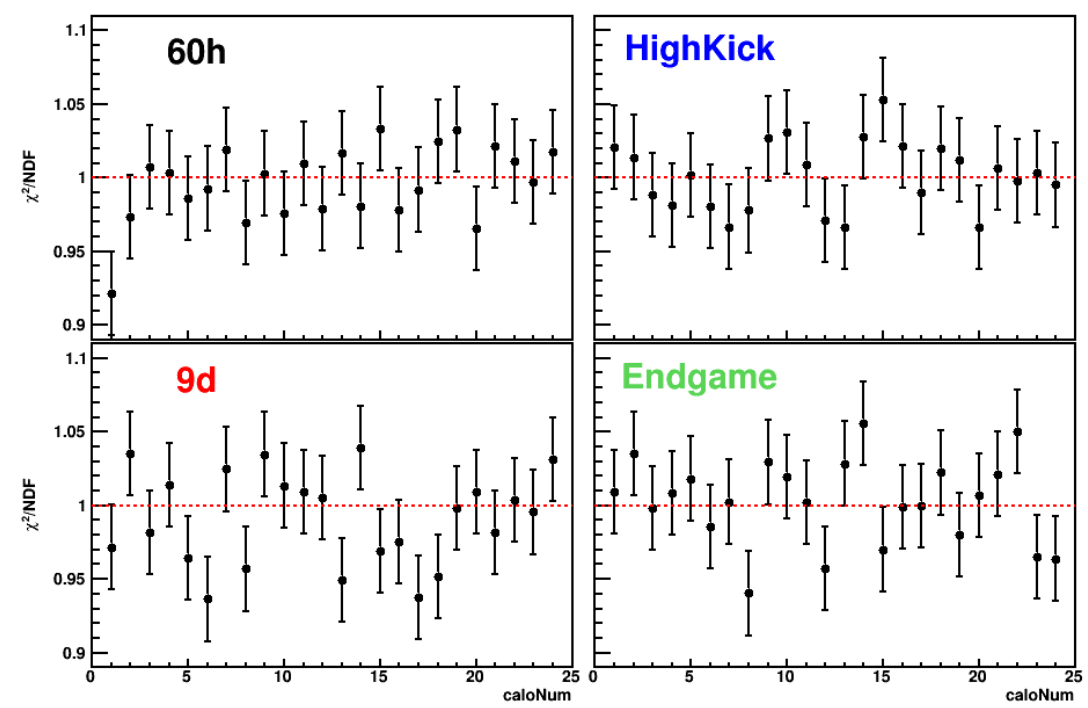

Figure 5·26: $\chi^{2} /$ NDF's versus calorimeter number for the Run 1 precession frequency analysis datasets. Red dashed lines are placed at $\chi^{2} / \mathrm{NDF}=1$ to aid the eye. No individual calorimeter fits are preferentially low or high when comparing across datasets.
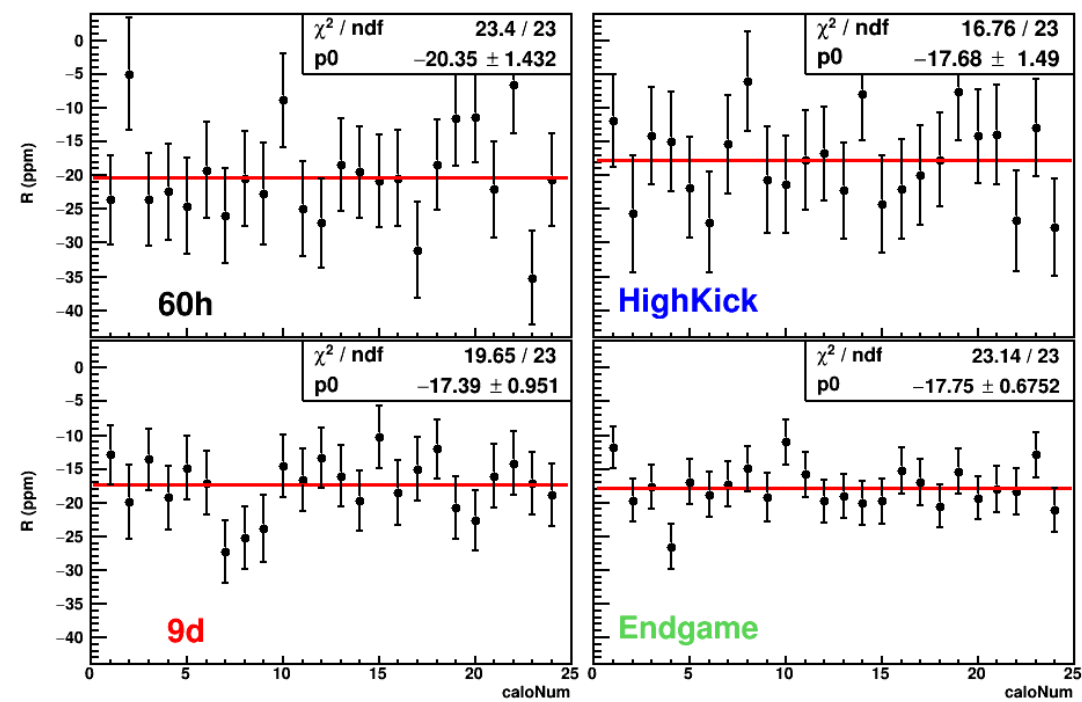

Figure 5·27: $R$ versus calorimeter number for the Run 1 precession frequency analysis datasets. The scale is the same on each of the plots. A straight line fit was performed on the fitted values, with the fit result shown in the upper right box as parameter $p_{0}$ in units of ppm. The different blinding in the $60 \mathrm{~h}$ dataset is noticeable. The higher precision fits to the 9d and Endgame datasets have correspondingly smaller error bars. 

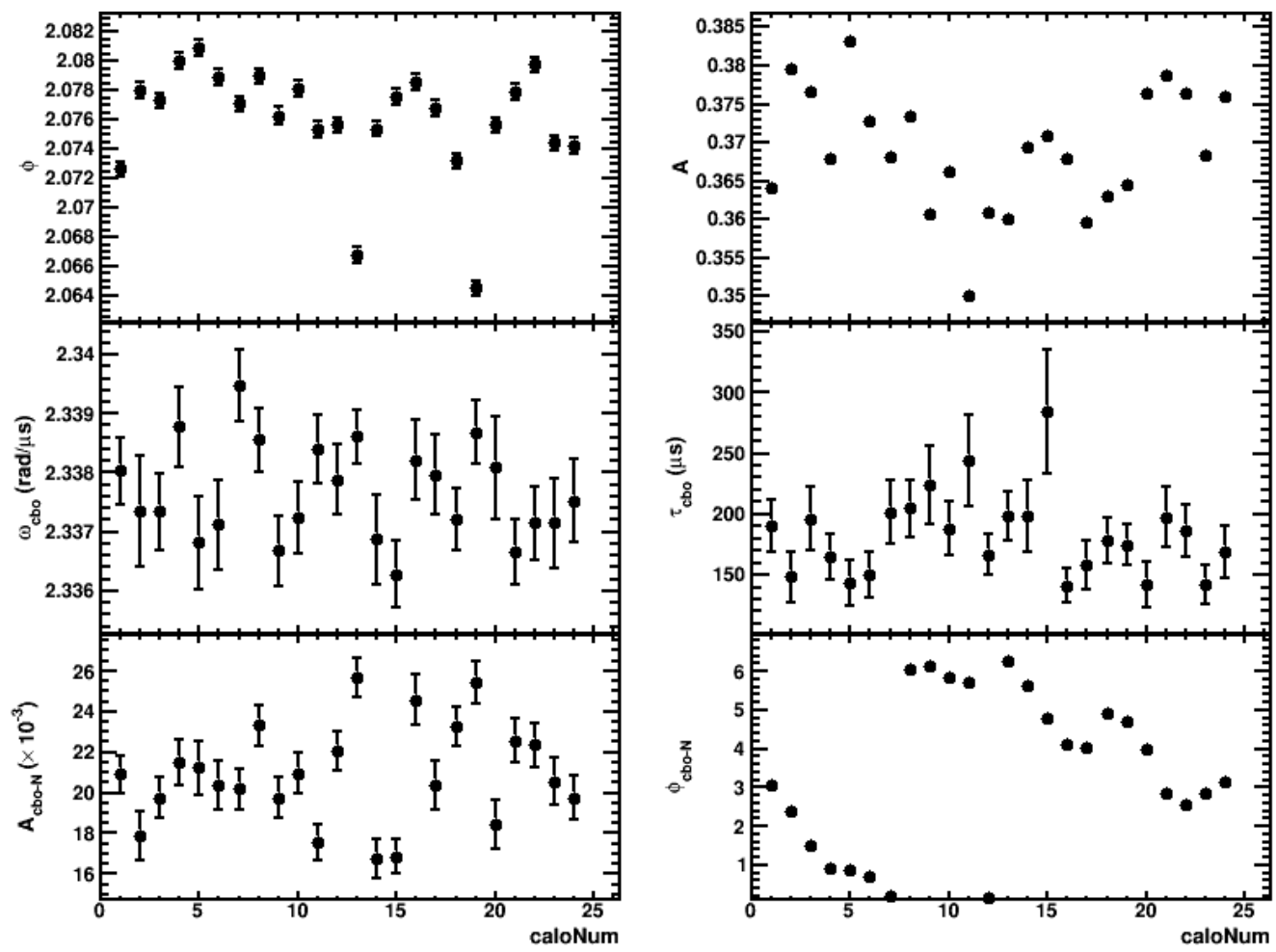

Figure 5.28: Endgame fit parameters versus calorimeter number. In the $g-2$ phase $\phi$ (top-left) two points lie below the others, corresponding to those calorimeters which sit behind tracker stations. The CBO phase $\phi_{c b o-N}$ (bottom-right) runs from $0-2 \pi$ around the ring. These plots are typical of all datasets, with small variations in the final fitted parameters.

consistent with some spread due to acceptance, with the phases running from $0-2 \pi$ around the ring as expected. The amplitudes of the CBO parameters are an order of magnitude larger than in the calorimeter sum fits. Because the phases vary around the ring, the $\mathrm{CBO}$ effect becomes reduced when adding up all the individual spectra from the calorimeters. In fact, while it is not always necessary to include the higher order CBO terms for good fits to the calorimeter sum data, there are many calorimeters which need the higher order terms for good fits. 

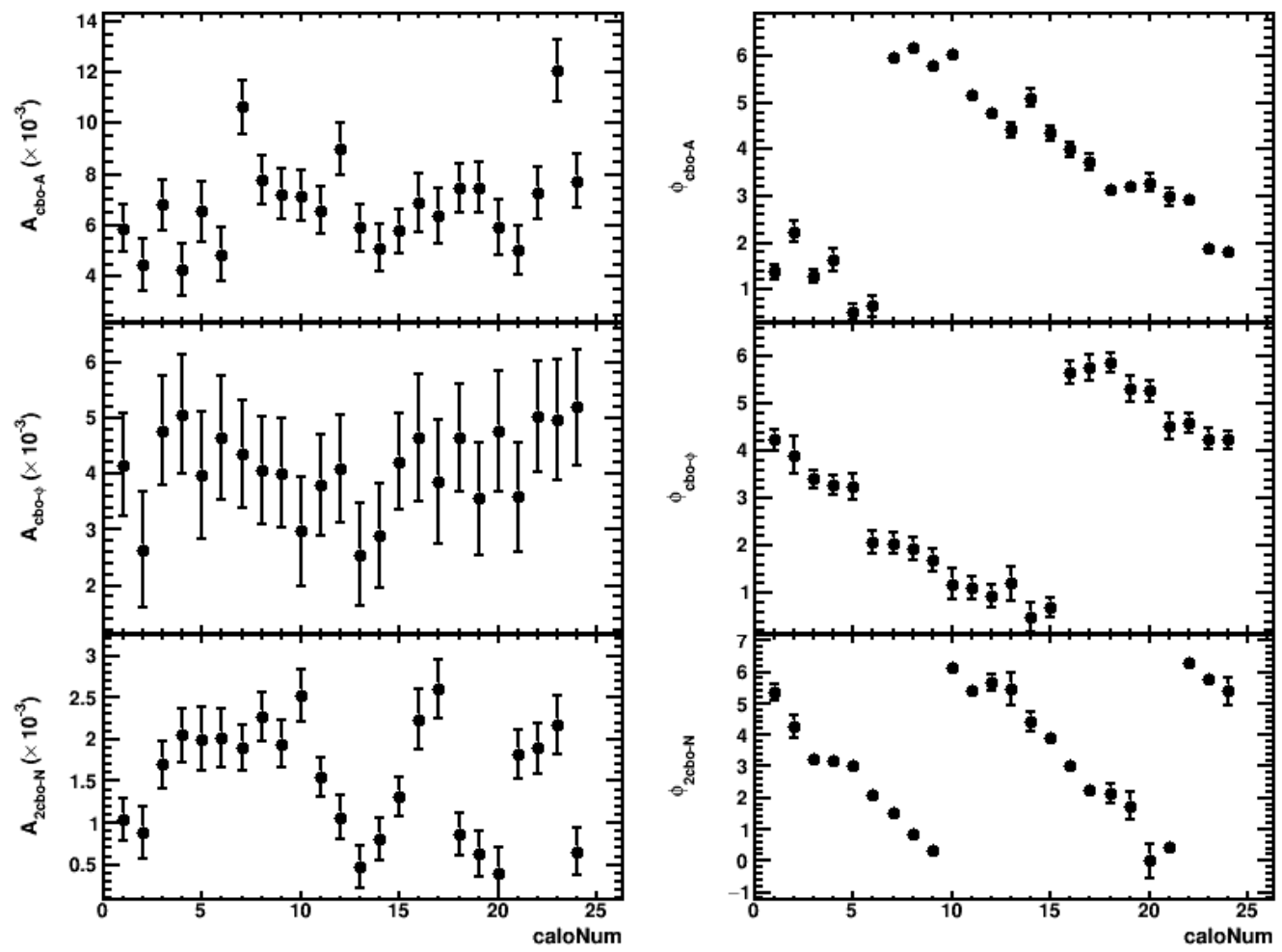

Figure 5·29: Additional Endgame fit parameters versus calorimeter number. The CBO phases $\phi_{c b o-A}$ and $\phi_{c b o-\phi}$ run from $0-2 \pi$ around the ring, while $\phi_{2 c b o-N}$ runs around twice. These plots are typical of all datasets, with small variations in the final fitted parameters.

\subsubsection{Scans over fit start time, fit end time, and energy threshold}

In order to determine if there are any deficiencies in the fit as a function of time, for instance if the $\mathrm{CBO}$ was modeled incorrectly, fits are performed with varying fit start and end times. If an effect is incorrectly modeled, the corresponding fit parameter will wander away from the statistically allowed deviation, as the effect grows stronger or weaker. Two examples of this are given in Figure 5.30. In the first case the CBO frequency was mis-measured when using a fixed CBO frequency model as opposed to a changing one, and in the second case the asymmetry was mis-measured when pileup was not subtracted from the data. In general, doing a fit start scan is a very useful tool in the precession frequency analysis beyond just verifying consistency of 
a fit parameter as a function of time, as it also provides hints as to what might be wrong with how the data are being handled.

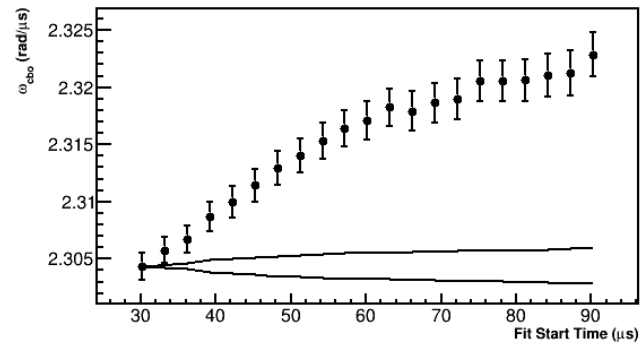

(a) The fit start scan for the fitted CBO frequency when a constant frequency model is included. The p-value of the first ratio fit in this case is 0.191 , indicating an acceptable fit. Only by performing a fit start scan or examining the fit residuals closely is the deficiency in the fit observed.

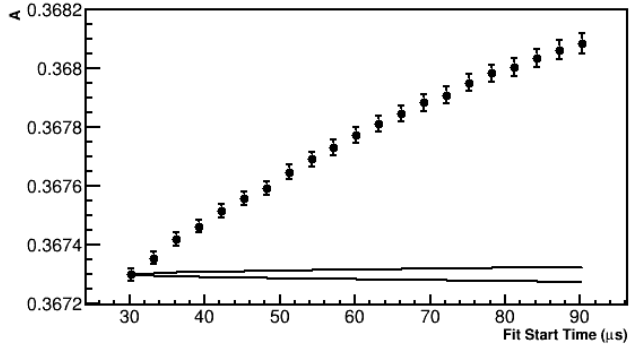

(b) The fit start scan for the fitted asymmetry when the pileup is not subtracted out. If the pileup is not properly accounted for, then decay positrons with lower asymmetries contaminate the observed decay time spectrum leading to a lower fitted asymmetry value. As the pileup diminishes the fitted asymmetry tends to its true value.

Figure 5·30: Examples of fit start scans when effects are improperly accounted for before or during fitting. The parabolic bands indicate the $1 \sigma$ allowed deviation in the fit parameters due to the changing statistics between the fits. Data are from the Endgame dataset.

The statistically allowed deviation between two sets of $g-2$ data, where one is a subset of the other, is given by 36

$$
\sigma_{d i f f}=\sqrt{\sigma_{2}^{2}-\sigma_{1}^{2}\left(2 \frac{A_{1}}{A_{2}} \cos \left(\phi_{1}-\phi_{2}\right)-1\right)},
$$

where the subscript 2 stands for the larger dataset while the subscript 1 stands for the smaller sub-dataset. This statistically allowed deviation depends both on the size of the datasets as well as their "analyzing powers," which come from the asymmetries and phases of the datasets. For fit start scans the analyzing powers are in general the 
same, such that the approximation

$$
\sigma_{d i f f} \approx \sqrt{\sigma_{2}^{2}-\sigma_{1}^{2}}
$$

can be made.

It should be noted that for fits with late fit start times, certain CBO parameters start to become unstable as the $\mathrm{CBO}$ effect becomes diminished in the data 17 . For instance, fits with start times at $100 \mu$ s are half a lifetime or more along the CBO effect and convergence is difficult to achieve. The values of unstable parameters were thus fixed to their starting fit values. These parameters typically included the CBO lifetime and most of the higher order $\mathrm{CBO}$ amplitudes and phases, though in some cases they could still be included out to late fit start times depending on the dataset.

Fit start time scans were performed from the default value of $30.2 \mu$ s up to $100.2 \mu \mathrm{s}$ in steps of $1 \mu \mathrm{s}$ for a total of 71 separate fits. In order to assist fit convergence, the final fit parameters from one fit were passed on as the starting parameters of the next. The $\chi^{2} / \mathrm{NDF}^{\prime} \mathrm{s}$ and fitted $R$ values as a function of fit start time for the four Run 1 precession frequency analysis datasets are shown in Figure 5·31. The errors on the individual points in the $\chi^{2}$ plots are given as

$$
\sigma_{\chi^{2} / N D F}=\sqrt{2 / N D F}
$$

where NDF changes as the fit start time is pushed later in time and bins are left out of the fit. The black parabolic bands indicate the $1 \sigma$ statistically allowed deviation given by Equation 5.32. As shown the goodness of fit for the four datasets are all consistent with fit start time, only wandering in and near the bands without diverging.

\footnotetext{
${ }^{17}$ The same applies to the VW effect when it is not time-randomized out of the data.
} 


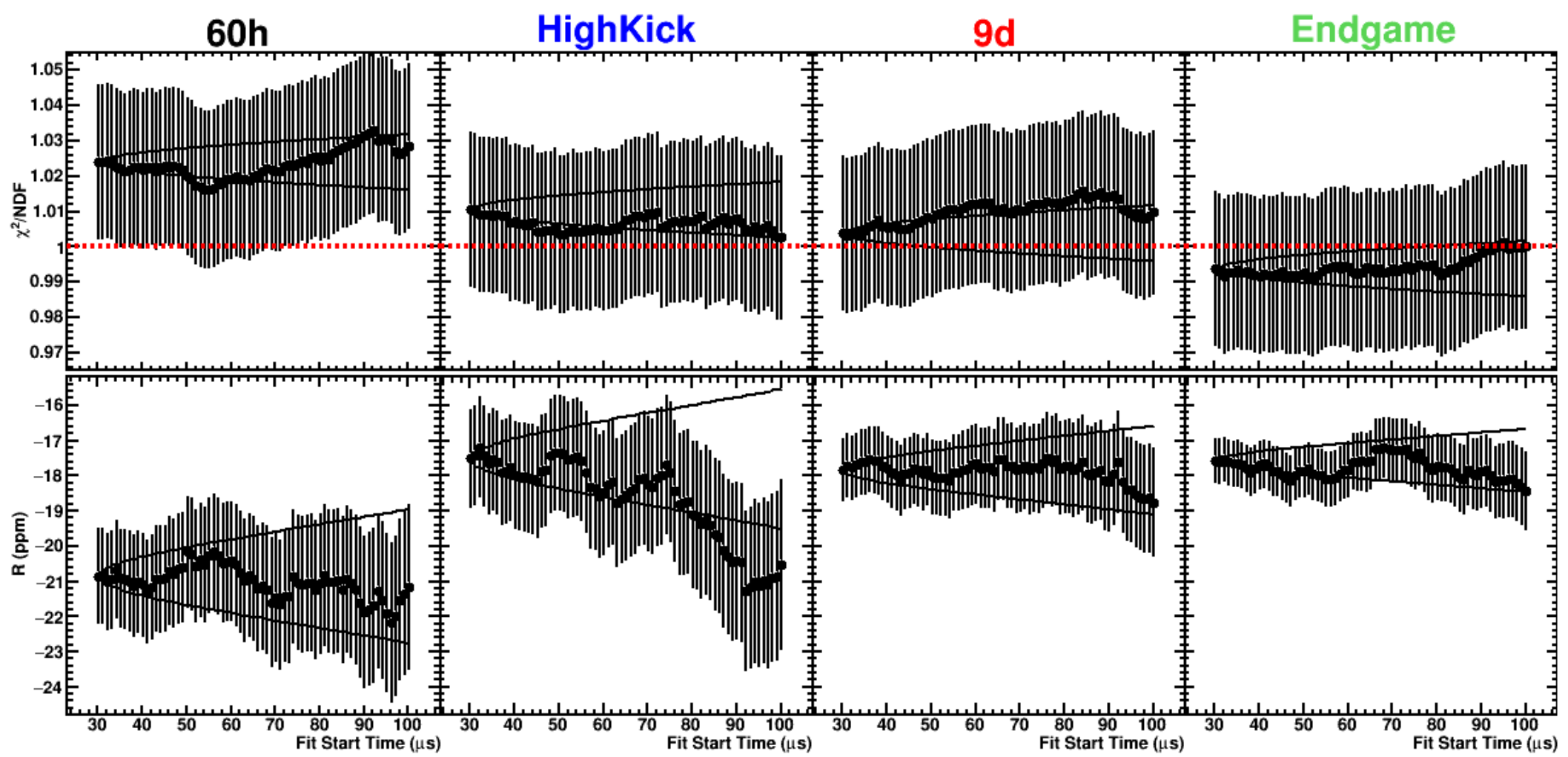

Figure 5·31: $\chi^{2} / \mathrm{NDF}$ (top) and $R$ (bottom) versus fit start time for the Run 1 precession frequency analysis datasets. Plots in the top and bottom rows are on the same scale respectively. The black parabolic bands represent the $1 \sigma$ statistically allowed deviation. The $\chi^{2}$ values are all consistent with 1 , for which a dashed red line has been overlaid. In all cases the fit points lie in and around the $1 \sigma$ statistical bands. 
$R$ is similarly consistent as a function of fit start time. The only dataset for which $R$ goes noticeably outside the bands is the HighKick. Since the deviation is less than $2 \sigma$ and it trends back towards the bands however, it does not appear particularly indicative of unaccounted effects in the data. In general, $R$ is very insensitive to changing fit start time, most likely due to the small correlations with other fit parameters. Figure $5 \cdot 32$ shows the fit start scan results for the other free parameters for the Endgame dataset. In all cases the fit parameters only wander in and near the bands, showing that all effects in the ratio data are properly accounted for.
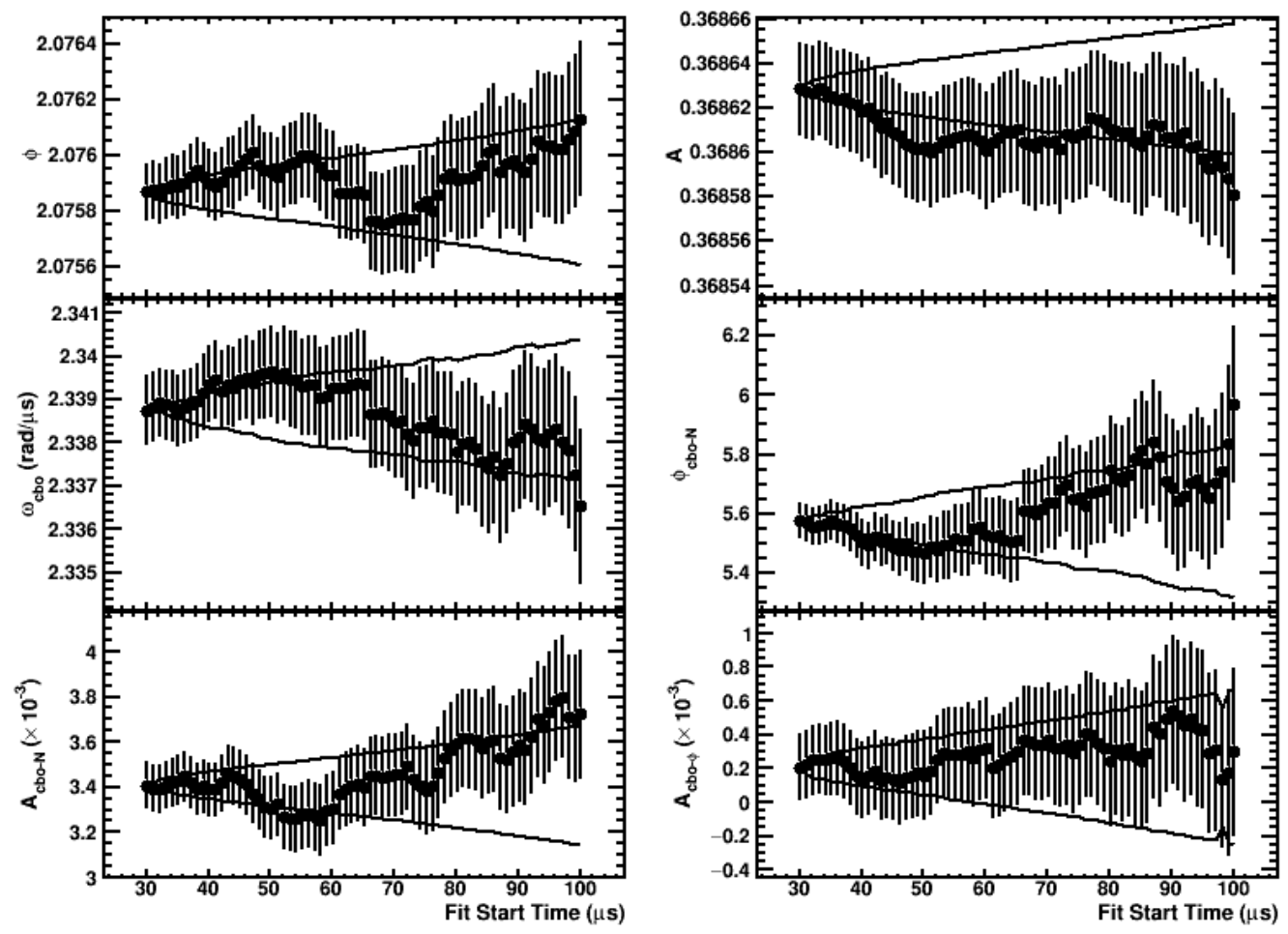

Figure 5.32: Fit start scans for free parameters in the Endgame dataset. Those parameters not shown here are fixed to their starting values over the course of the scan, as at late times they can be unstable as the $\mathrm{CBO}$ effects die away.

For fit end scans, all of the same methods and conclusions apply. In general fit end scans are both less dangerous and more stable than fit start scans, as the amount of data being removed from the fit is relatively small and effects like the $\mathrm{CBO}$ are 


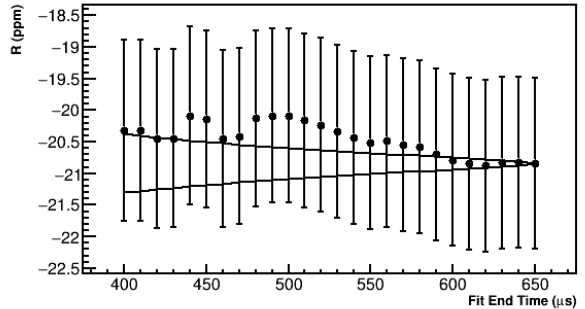

(a) $60 \mathrm{~h}$ dataset.

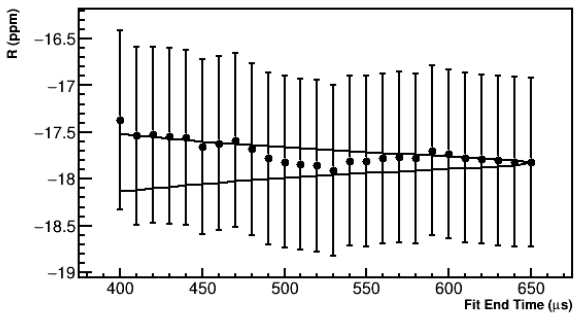

(c) $9 \mathrm{~d}$ dataset.

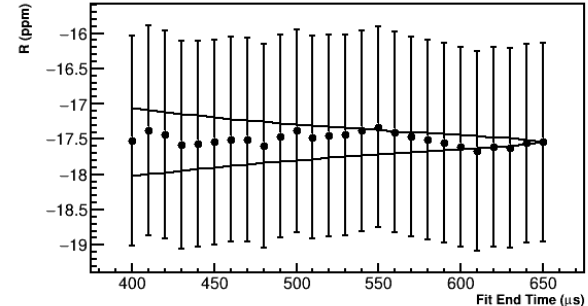

(b) HighKick dataset.

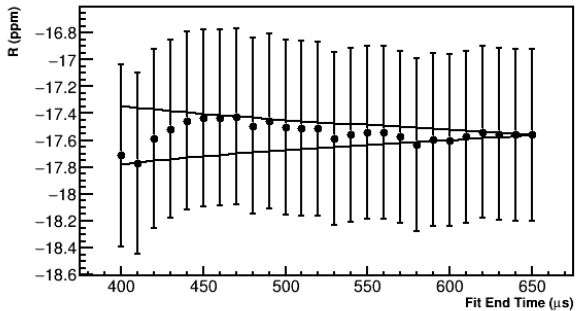

(d) Endgame dataset.

Figure 5.33: $R$ versus fit end time for the Run 1 precession frequency analysis datasets. The fit points lie in and around the $1 \sigma$ statistical bands.

largest at the beginning of the fill. While these fit end scans in a T-Method fit can probably be ignored in favor of the fit start scans, it's important to check that they satisfy the statistical deviations in the Ratio Method as the ratio data errors grow larger with less data [119]. Fit end time scans were performed from $650 \mu \mathrm{s}$ to $400 \mu \mathrm{s}$ in steps of $10 \mu \mathrm{s}$, corresponding to 26 separate fits. As in the fit start time scans, fit results from the end of one fit were passed on as the starting parameters to the next. $R$ values for fit end scans for the Run 1 precession frequency analysis datasets are shown in Figure $5 \cdot 33$. As shown the $R$ values are within and near the bands.

Similarly to fit start and end time scans, it is worthwhile to verify that $R$ is consistent regardless of the energy threshold applied to the decay positron time spectrum. The energy threshold was varied from $1.2 \mathrm{GeV}$ to $2.2 \mathrm{GeV}$ in steps of $50 \mathrm{MeV}$ corresponding to 21 separate fits. The fitted $R$ values for the four Run 1 precession 


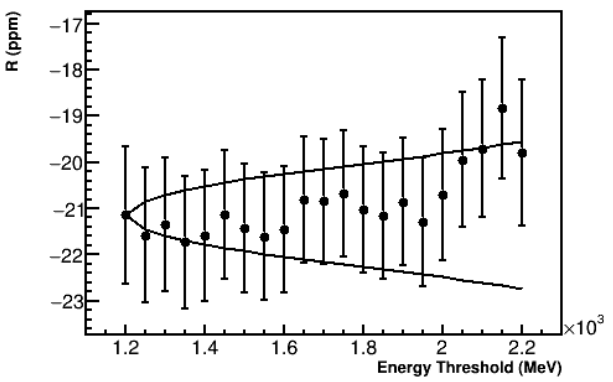

(a) $60 \mathrm{~h}$ dataset.

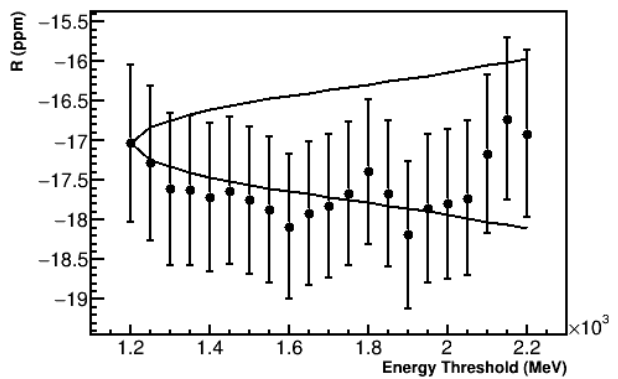

(c) 9d dataset.

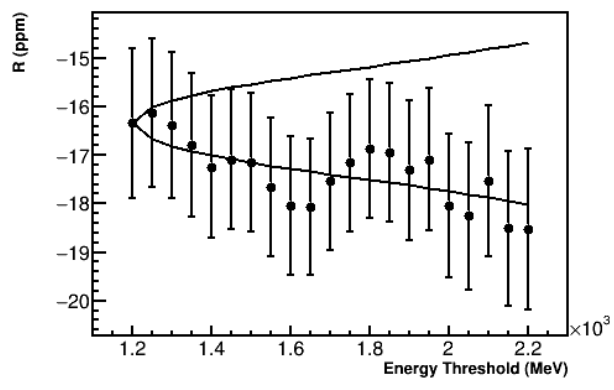

(b) HighKick dataset.

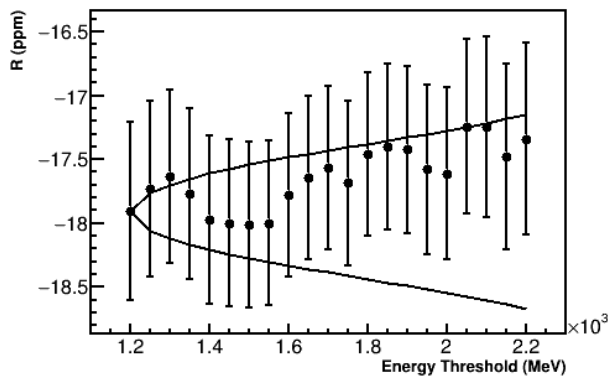

(d) Endgame dataset.

Figure 5.34: $R$ versus energy threshold for the Run 1 precession frequency analysis datasets. The fit points lie in and around the $1 \sigma$ statistical bands.

frequency analysis datasets are shown in Figure $5 \cdot 34$. The statistical bands are the same as those defined in Equation 5.31, where now the analyzing power part of the equation plays a larger role as the asymmetries and phases of the different fit points are significantly different. As shown there are no major deviations in the fitted $R$ values.

\subsubsection{Fits to bunch number}

Eight distinct and separate bunches of muons are sent to the E989 experiment within the accelerator timing structure as described in Section 2.3. Differences upstream and at injection mean that the phase space of the stored beam varies slightly with bunch 


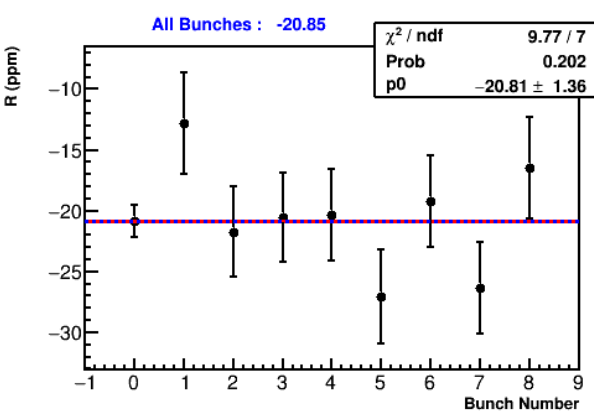

(a) $60 \mathrm{~h}$ dataset.

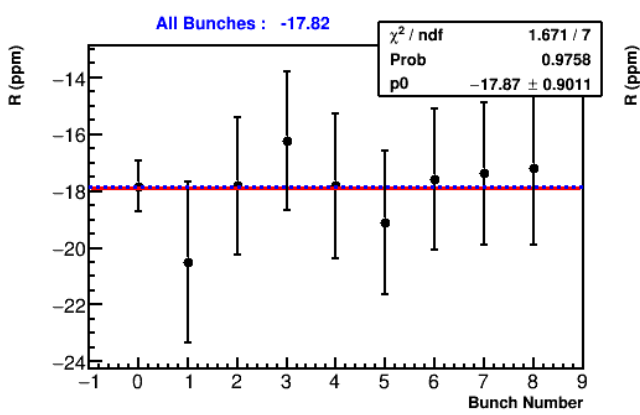

(c) 9 d dataset.

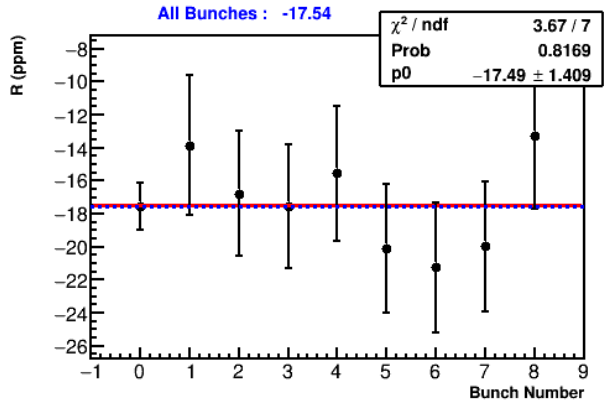

(b) HighKick dataset.

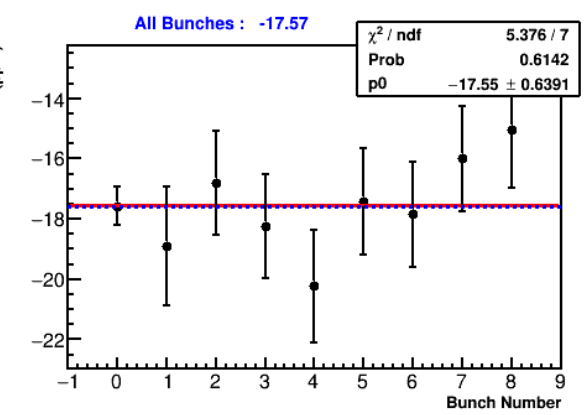

(d) Endgame dataset.

Figure 5·35: $R$ versus bunch number for the Run 1 precession frequency analysis datasets. Bunch number 0 corresponds to the data from all bunches added together. The blue dashed line intersects the bunch number 0 point, and it's value is displayed in the top left of each plot. The red line corresponds to a fit to bunches $1-8$, with $p_{0}$ being the fit parameter. In all cases the fitted $R$ value to bunches $1-8$ is nearly identical to the all-bunches result.

number. In order to verify that the treatment of fit parameters was correct regardless of the bunch number and thereby improve confidence in the results, the bunches were fit individually. Figure $5 \cdot 35$ shows the fitted $R$ values for the eight individual bunches alongside the bunch-sum result, for the Run 1 precession frequency analysis datasets. No systematic variation in $R$ as a function of bunch number was observed. When the eight individual bunches were fit to a straight line, they were found to be consistent with the bunch-sum result. 


\begin{tabular}{lcccc}
\hline \multicolumn{5}{c}{ Random Seed Fit Results } \\
\hline \hline Dataset & $\chi^{2}$ Mean & $R$ Mean & $R$ RMS & $R$ Error on Mean \\
\hline 60h & 0.999 & -20.5562 & 0.3443 & 0.0487 \\
HighKick & 1.001 & -17.4755 & 0.4226 & 0.0598 \\
$9 \mathrm{~d}$ & 0.999 & -17.7182 & 0.2118 & 0.0300 \\
Endgame & 1.002 & -17.3406 & 0.1249 & 0.0177 \\
\hline
\end{tabular}

Table 5.8: Random seed fit results to the four Run 1 precession frequency analysis datasets. The $\chi^{2}$ means are consistent with 1 . As a reminder the $60 \mathrm{~h}$ dataset used a different blinding than the other three, hence the significantly different $R$ mean. Units are in ppm.

\subsubsection{Fits to many random seeds}

While the single seed fit results presented earlier indicate good fits and well understood parameters, it is always a good idea to fit other random seeds in case the single seed results ended up on an outlier. Doing so not only improves the confidence of the result, but also gives a more central $R$ value to quote as being closer to the 'true' $R$ value of the dataset. Figure 5.36 gives the $\chi^{2}$ and $R$ distributions for fits to 50 different random seeds for the four datasets. As shown the $\chi^{2}$ distributions are centered around 1 as expected. Table 5.8 compares the random seed fit results between the different datasets. The means for the $R$ distributions of the datasets which shared the same blinding string (HighKick, 9d, Endgame) are statistically consistent with one another, with differences on the order of several hundreds of ppb, well within the statistical errors of $600 \mathrm{ppb}$ or greater ${ }^{18}$.

\footnotetext{
${ }^{18}$ The errors on the mean are calculated as $\sigma_{\mu}=\mathrm{RMS} / \sqrt{N}$, where $N$ is the number of random seeds. These errors come from the randomization of cluster times before fitting, and have no bearing on statistical consistency when comparing different datasets.
} 

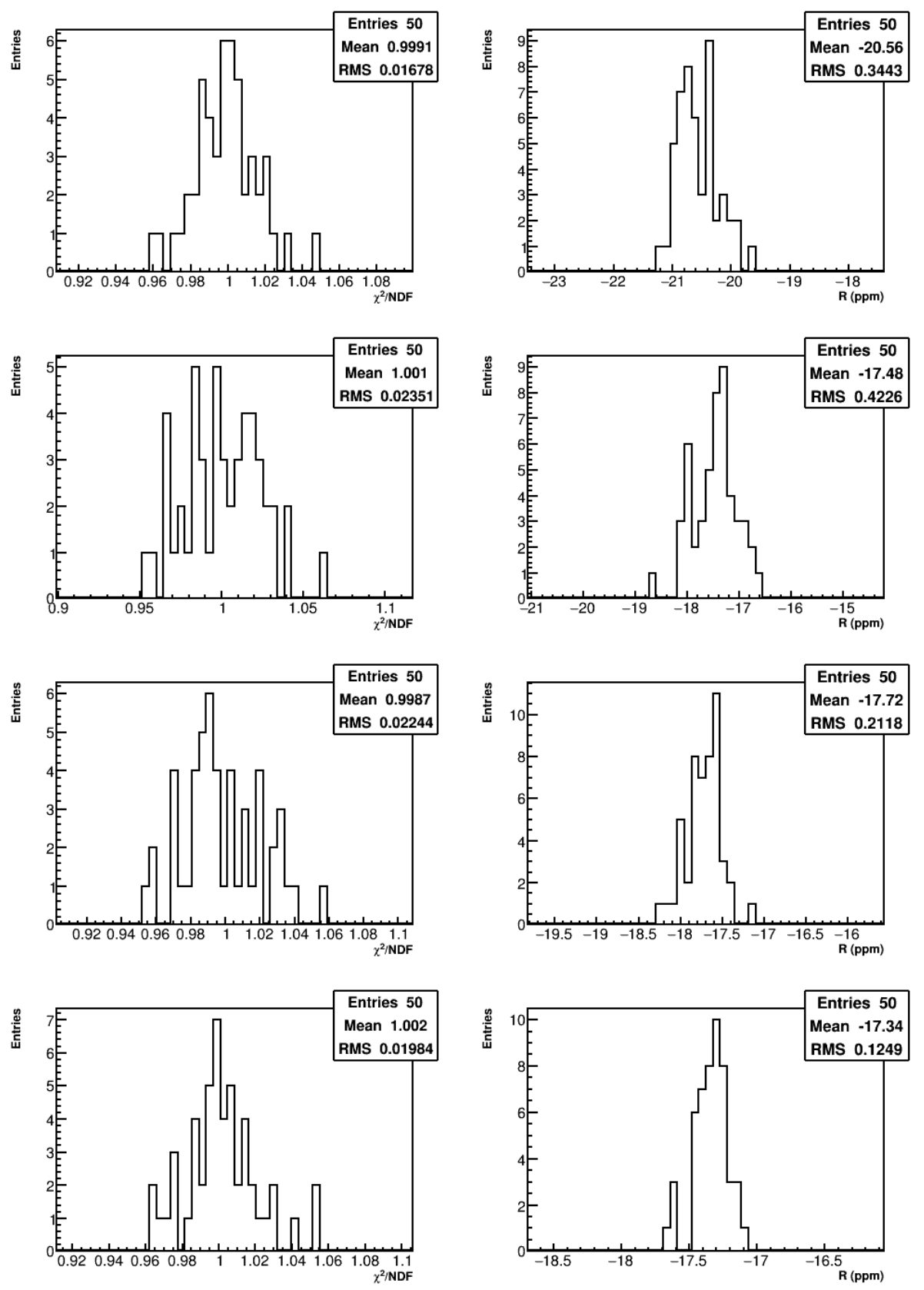

Figure 5.36: $\chi^{2}$ and $R$ values for fits to 50 different random seeds for the Run 1 precession frequency analysis datasets. Plots for the 60h, HighKick, 9d, and Endgame dataset are arrayed from top to bottom. The $\chi^{2}$ distributions are nicely centered around 1 which is to be expected if the randomized data are properly distributed and fit correctly. The statistics box in the $\mathrm{R}$ plots have units of ppm for the means and RMS' of the distributions. 


\begin{tabular}{lc}
\hline \multicolumn{2}{c}{ Target $\omega_{\boldsymbol{a}}$ Systematic Uncertainties } \\
\hline \hline Source of uncertainty & E989 Goal (ppb) \\
\hline Pileup & 40 \\
Gain changes & 20 \\
CBO & 30 \\
Lost muons & 20 \\
E-field and pitch corrections & 30 \\
Other & 30 \\
\hline Quadrature sum & $\sim 70$ \\
\hline
\end{tabular}

Table 5.9: Target systematic errors in the precession frequency measurement for the E989 experiment [54].

\subsection{Systematic errors}

Evaluating the systematic errors which bias the $\omega_{a}$ frequency extraction is a major part of the precession frequency analysis. Systematic errors typically stem from sources which introduce time-dependent phases over the course of a fill,

$$
\cos \left(\omega_{a} t+\phi(t)\right) \rightarrow \cos \left(\left(\omega_{a}+\phi_{1}\right) t+\phi_{0}+\phi_{2} t^{2}+\ldots\right),
$$

where $\phi(t)$ can in general be expanded to higher orders. Such time-dependent phases from effects which change early-to-late within the fill can thus result in mis-measured values for $\omega_{a}$, with no indications that the extracted values are biased. Sources of systematic uncertainty can be separated into several general categories. These include systematic errors in the pileup subtraction, gain corrections, CBO fit model, muon losses, and E-field and pitch corrections, among others. Table 5.9 gives the target systematic uncertainties for the E989 experiment, which has the goal of a $70 \mathrm{ppb}$ systematic error on the precession frequency measurement. This represents a three to four-fold improvement over the E821 experiment systematic errors which were of order $210-310 \mathrm{ppb}$, depending on running period [36]. 
Run 1 of E989 consisted of multiple separate datasets with distinct running conditions, necessitating the need for independent systematic uncertainty evaluations. The calculations of the various systematic errors typically involve varying the relevant histogram or analysis parameters, and observing the changes in the final fitted $R$ values. Preliminary estimates are given for systematic errors which are produced by other analysis groups. These include errors from the phase of the lost muons, the E-field and pitch corrections, and the vertical beam movement due to the bad quadrupole resistors.

\subsubsection{Pileup systematic errors}

The pileup background oscillates at $\omega_{a}$ and has a different phase than the main $g-2$ signal, which by extension implies a strong effect on the extracted frequency value. If the subtracted pileup spectrum is mis-constructed in any way, there will be a systematic error on $R$, as described in Section 5.2.1. The pileup systematic error can be separated into two parts, those being the errors on the amplitude and phase of the correction. In order to evaluate the systematic errors due to mis-construction of the amplitude or the phase, the uncertainties in them need to be estimated along with the corresponding sensitivities of $R$. Table 5.1 gives the default values of the pileup construction parameters $\{$ ADT, SDT, SGT, C $\}$. How these parameters feed into the amplitude and phase systematic errors will be discussed in turn, and the overall errors calculated for the different Run 1 datasets.

As a reminder, the default values used for the ADT and SDT were each $5 \mathrm{~ns}$. In order to calculate the systematic dependence on the choice of ADT or SDT, the SDT parameter was scanned from $5 \mathrm{~ns}$ to $10 \mathrm{~ns}$ in steps of $1 \mathrm{~ns}$. This was done with and without the pileup amplitude scaling procedure as described in Section 5.2.1, which as a reminder applies a default pileup amplitude multiplier of approximately 1.03 for each dataset. This amplitude scaling procedure was automated in the analysis such 
that the pileup spectra amplitude was adjusted to the appropriate level regardless of the choice of pileup spectra parameters. The results of the study for the $9 \mathrm{~d}$ dataset are shown in Figures $5 \cdot 37$ and $5 \cdot 38$. In the case where there was no automatic amplitude scaling applied, there is a clear minimum in the $\chi^{2}$ results and a steep slope in $R$, corresponding to a large sensitivity of $R$ to the choice of SDT. In the case where the automatic amplitude scaling was applied however, the minimum in the $\chi^{2}$ results has disappeared, and there is no clear effect on $R$. Applying the automatic pileup amplitude scaling produces nearly identical pileup spectra with no clear trend in $R$ regardless of the choice of SDT (and by extension ADT). Any systematic error due to the choice of these two parameters can then be subsumed into the direct pileup amplitude error itself, discussed below. It should be noted that the choices of ADT and SDT are largely irrelevant barring statistics, as the automatic amplitude scaling procedure can always account for any differences between the two.

In order to calculate the systematic dependence on the choice of SGT (default value of $10 \mathrm{~ns}$ ), the SGT parameter was scanned from $10 \mathrm{~ns}$ to $20 \mathrm{~ns}$ in steps of $1 \mathrm{~ns}$. The results of the study with the automatic pileup amplitude scaling applied are shown in Figure 5.39, Just as in the SDT scan with the automatic pileup amplitude scaling, there is no minimum in the $\chi^{2}$ results, the sensitivity of $R$ to the value of SGT is negligible, and the pileup spectra for the various choices of SGT are nearly identical. Therefore again, any systematic error due to the choice of SGT is subsumed into the pileup amplitude error. This is to be expected as there are no major changes in the hit time spectrum at short time scales, $\mathcal{O}(10 \mathrm{~ns})$. If the SGT value used was significantly larger, then a systematic effect might arise.

In order to evaluate the pileup amplitude systematic error, multipliers were applied to the pileup spectra from 0.9 to 1.1 in steps of 0.01 , bracketing the default pileup scaling of $\sim 1.03$ mentioned before. The results of the study for the $9 \mathrm{~d}$ dataset are 


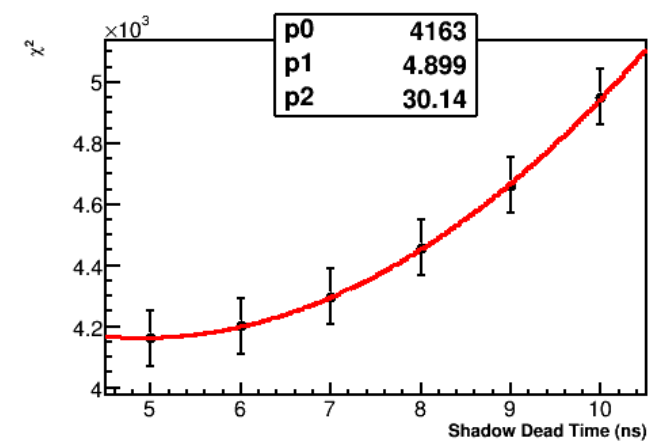

(a) $\chi^{2}$ versus SDT. The parabolic fit equation used was $y=p_{2}\left(x-p_{1}\right)^{2}+p_{0}$.

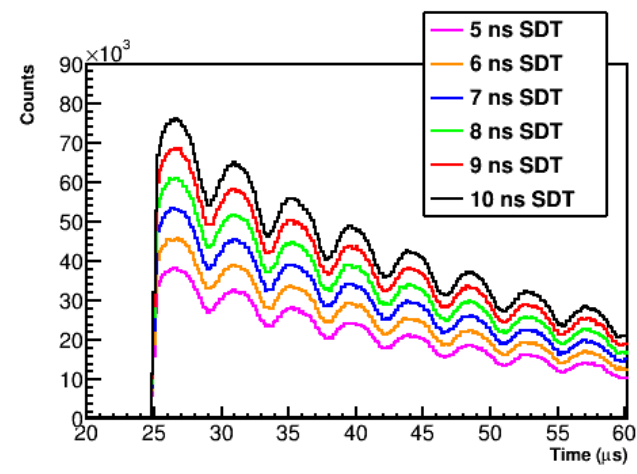

(c) The pileup time spectrum for different choices of SDT.

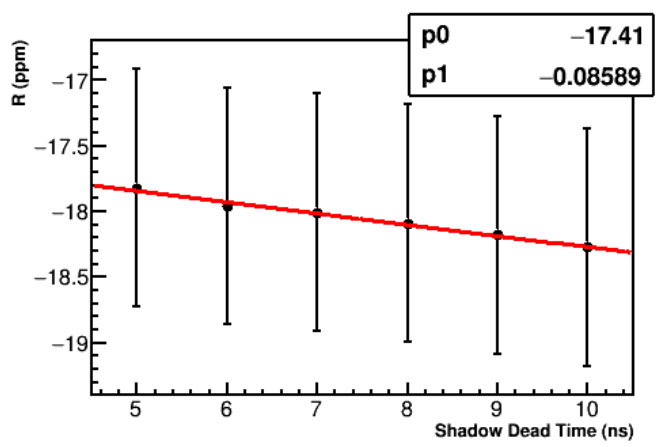

(b) $R$ versus SDT. The parameter $p_{1}$ characterizes the sensitivity of $R$ to the choice of SDT, with units in $\mathrm{ppm} / \mathrm{ns}$.

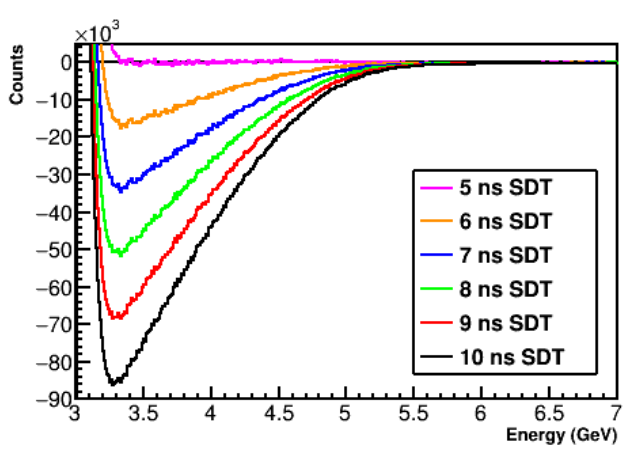

(d) The corrected energy spectrum above $3 \mathrm{GeV}$ for different choices of SDT.

Figure 5·37: Shadow dead time scan results without automatic pileup amplitude scaling. A clear minimum in the $\chi^{2}$ plot is seen near $5 \mathrm{~ns}$ corresponding to the choice of SDT $=$ ADT, and a large sensitivity for $R$ is observed. A larger choice of SDT leads to a greater estimation of the pileup, which as shown in the energy spectra plot leads to a corresponding over-subtraction at energies where pileup pulses dominate. Data are from the $9 \mathrm{~d}$ dataset. 


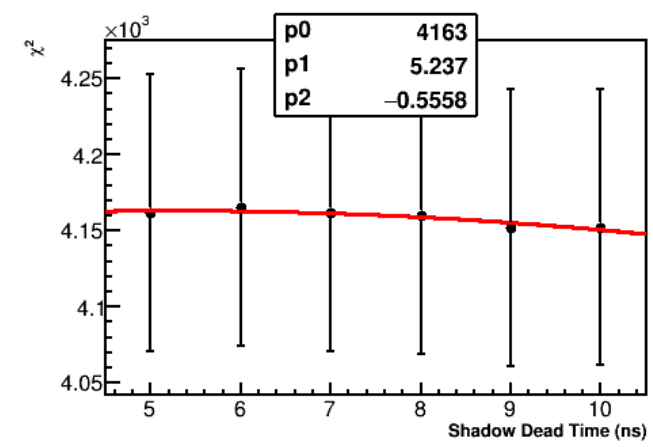

(a) $\chi^{2}$ versus SDT. The parabolic fit equation used was $y=p_{2}\left(x-p_{1}\right)^{2}+p_{0}$.

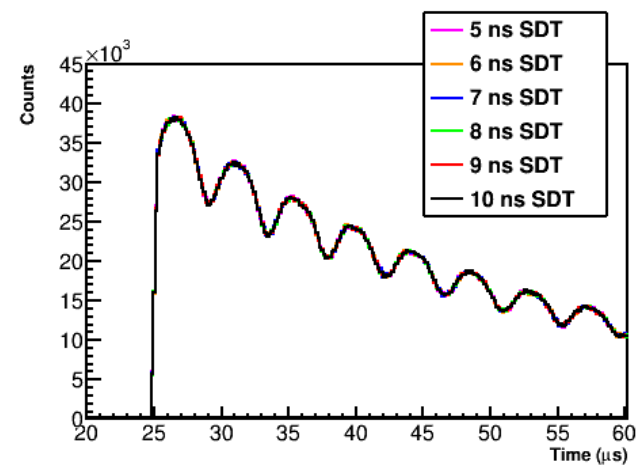

(c) The pileup time spectrum for different choices of SDT.

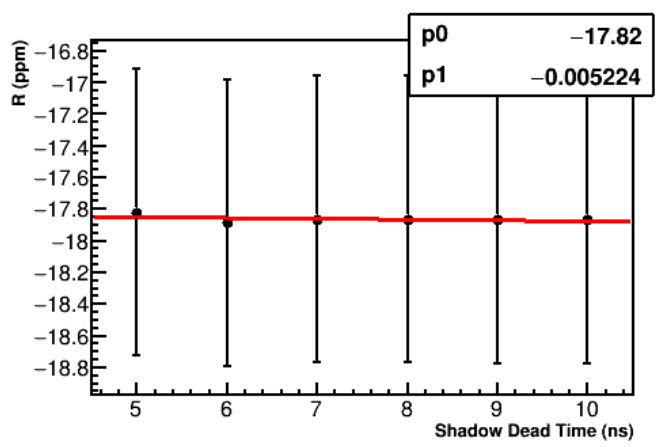

(b) $R$ versus SDT. The parameter $p_{1}$ characterizes the sensitivity of $R$ to the choice of SDT, with units in $\mathrm{ppm} / \mathrm{ns}$.

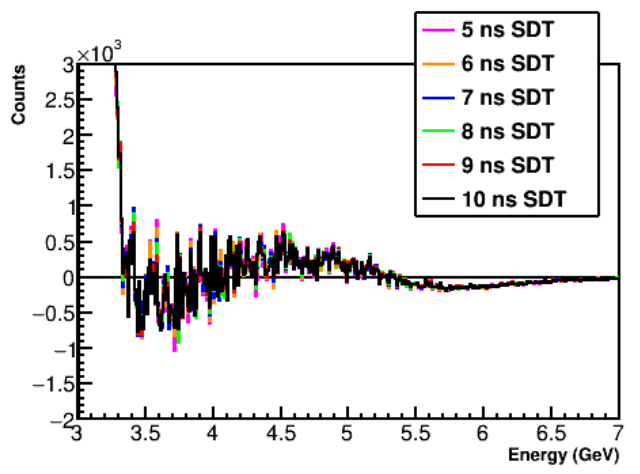

(d) The corrected energy spectrum above $3 \mathrm{GeV}$ for different choices of SDT.

Figure 5.38: Shadow dead time scan results with automatic pileup amplitude scaling. No clear minimum is observed in the $\chi^{2}$ plot, and the sensitivity for $R$ is small. With the automatic amplitude scaling applied, the time and energy spectra are nearly identical and lie on top of each other. The lack of clear minimum in the $\chi^{2}$ plot and no clear sensitivity in $R$ indicate that there is no real systematic error due to the choice of SDT. Data are from the 9d dataset. 


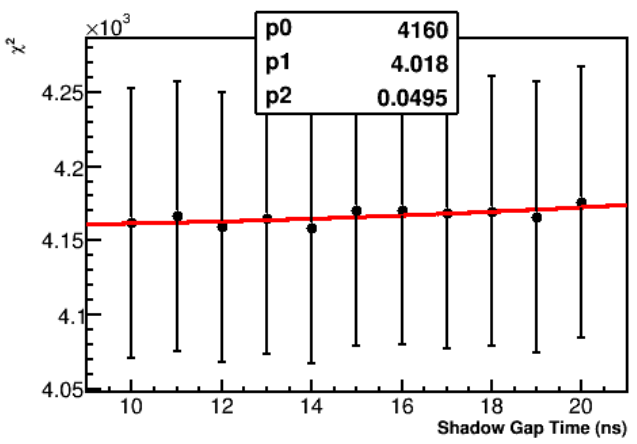

(a) $\chi^{2}$ versus SGT. The parabolic fit equation used was $y=p_{2}\left(x-p_{1}\right)^{2}+p_{0}$.

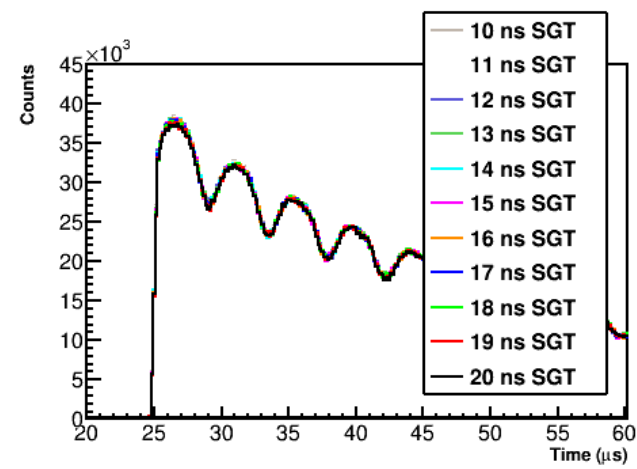

(c) The pileup time spectrum for different choices of SGT.

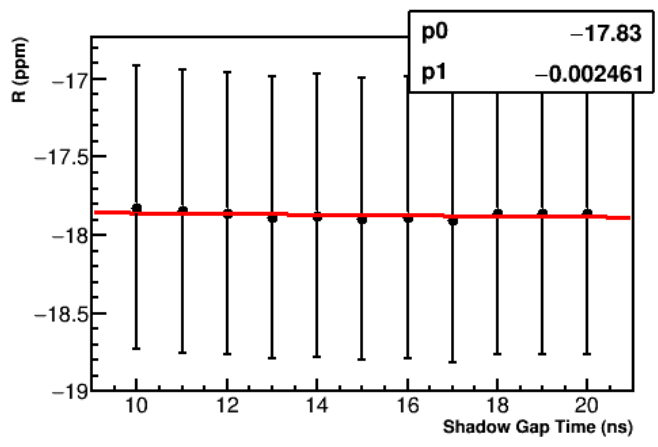

(b) $R$ versus SGT. The parameter $p_{1}$ characterizes the sensitivity of $R$ to the choice of SGT, with units in $\mathrm{ppm} / \mathrm{ns}$.

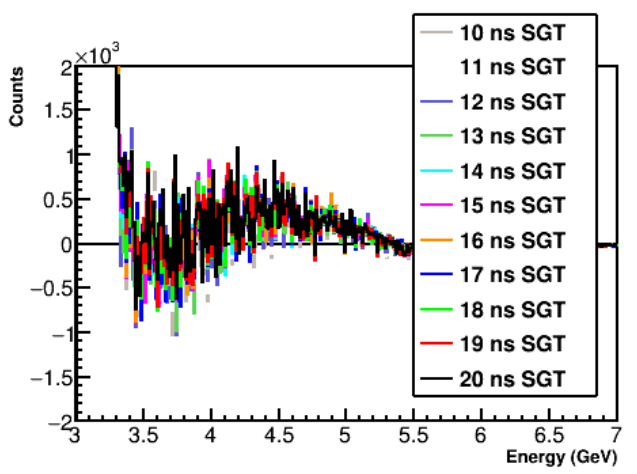

(d) The corrected energy spectrum above $3 \mathrm{GeV}$ for different choices of SGT.

Figure 5.39: Shadow gap time scan results with automatic pileup amplitude scaling. No clear minimum is observed in the $\chi^{2}$ plot, and the trend for $R$ isn't clear, with points fluctuating above and below the fit curve. With the automatic amplitude scaling applied, the time and energy spectra lie on top of each other. The lack of clear minimum in the $\chi^{2}$ plot and negligible sensitivity in $R$ indicate that there is no real systematic error due to the choice of SGT. Data are from the $9 \mathrm{~d}$ dataset. 
shown in Figure 5.40. There is a clear minimum near 1 in the $\chi^{2}$ results and $R$ is sensitive to the multiplier. The systematic error on $R$ is calculated as

$$
\delta R=\sigma_{P_{m}} \times \frac{d R}{d P_{m}}
$$

where $P_{m}$ is the value of the pileup multiplier. The uncertainty $\sigma_{P_{m}}$ is calculated as the width of the fitted parabola in the $\chi^{2}$ plot, defined as the change in $P_{m}$ which increases the minimum $\chi^{2}$ by 1 . This is calculated as

$$
\sigma_{P_{m}}=\sqrt{\frac{2}{f^{\prime \prime}\left(\chi^{2}\right)}}=\frac{1}{\sqrt{p_{2}}}
$$

where $p_{2}$ is the fit parameter as given in the $\chi^{2}$ plot. The sensitivities of $R$ to the pileup multiplier, uncertainties in the pileup amplitude, and final corresponding systematic errors for the Run 1 precession frequency analysis datasets are given in Table 5.10 . As shown in the table, the uncertainties on the pileup amplitude are of order 2 to $5 \%$, while the systematic errors on $R$ are on the order of 10 to $20 \mathrm{ppb}$, depending on dataset. It should be noted that the default automatic pileup multiplier of $\sim 1.03$ does not necessarily correspond to the minimum in the $\chi^{2}$ plot, but is still within $1 \sigma$ of 1 or the minimum; except for the Endgame dataset, where it is closer to $2 \sigma^{19}$.

The pileup phase error can result from improper reconstruction of the pileup pulse times or energies. The error due to the former is calculated by applying time-shifts to $t_{\text {doublet }}$ as given in Equation 5.3. Doing this artificially applies a phase shift to the pileup time spectrum. The data are then re-fit for $R$ with the shifted pileup spectra. Figure $5 \cdot 41$ shows the results for the $9 \mathrm{~d}$ dataset with applied time-shifts between $-10 \mathrm{~ns}$ and $10 \mathrm{~ns}$ in steps of $1 \mathrm{~ns}$. Although a sensitivity of $R$ to the value of the timeshift is observed, there is no clear minimum in the $\chi^{2}$ results, implying the data are fit

\footnotetext{
${ }^{19}$ Monte-Carlo tests with various random seeds showed this minimum fluctuating above and below 1. The distance from 1 therefore is not a good measure for the uncertainty in the pileup amplitude, compared to the width of the $\chi^{2}$ parabola fit.
} 


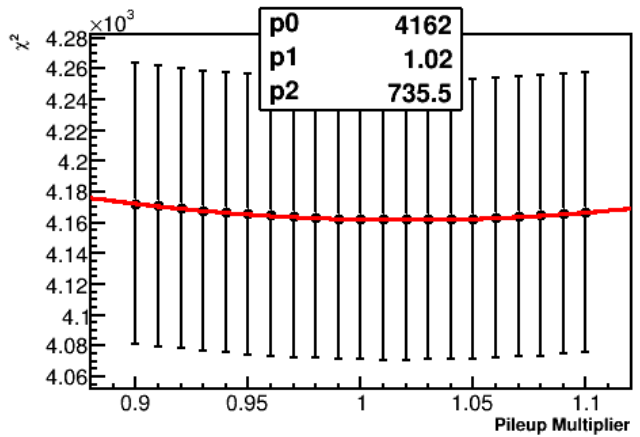

(a) $\chi^{2}$ versus pileup multiplier. The parabolic fit equation used was $y=$ $p_{2}\left(x-p_{1}\right)^{2}+p_{0}$. While the trend may initially appear somewhat flat, there is indeed a very clear minimum in the results when error bars are removed.

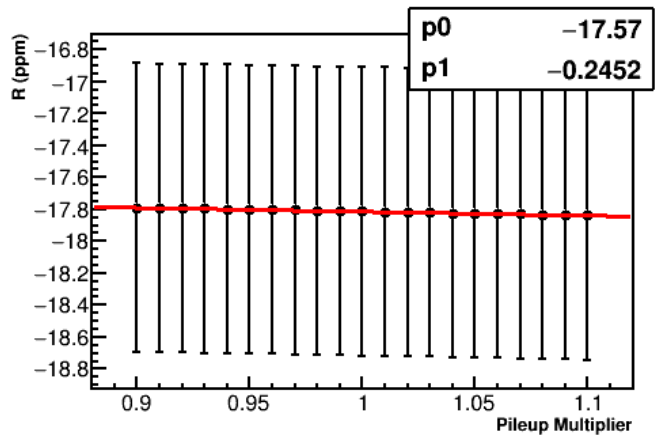

(b) $R$ versus pileup multiplier. The parameter $p_{1}$ gives the sensitivity of $R$ to the value of the pileup multiplier, with units in ppm.

Figure 5·40: Pileup multiplier scan. Data are from the $9 \mathrm{~d}$ dataset.

\begin{tabular}{lcccc}
\hline \multicolumn{4}{c}{ Systematic Error due to Pileup Amplitude } \\
\hline \hline Dataset & $d R / d P_{m}$ & $\sigma_{P_{m}}$ & $P_{m_{\min }}$ & $\boldsymbol{\delta} \boldsymbol{R}$ \\
\hline 60h & -419.3 & 0.053 & 0.993 & $\mathbf{2 2 . 2}$ \\
HighKick & -372.8 & 0.051 & 0.997 & $\mathbf{1 9 . 0}$ \\
9d & -245.2 & 0.037 & 1.020 & $\mathbf{9 . 0}$ \\
Endgame & -335.3 & 0.028 & 0.985 & $\mathbf{9 . 4}$ \\
\hline
\end{tabular}

Table 5.10: Systematic error due to the pileup amplitude, in the Ratio Method fits for the Run 1 precession frequency analysis datasets. The bold column gives the systematic errors on $R$. Units for $d R / d P_{m}$ and $\delta R$ are in ppb. 


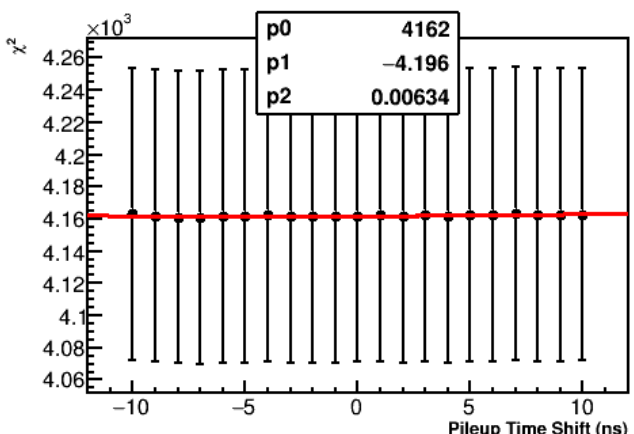

(a) $\chi^{2}$ versus pileup time-shift. There is no clear minimum in the plot.

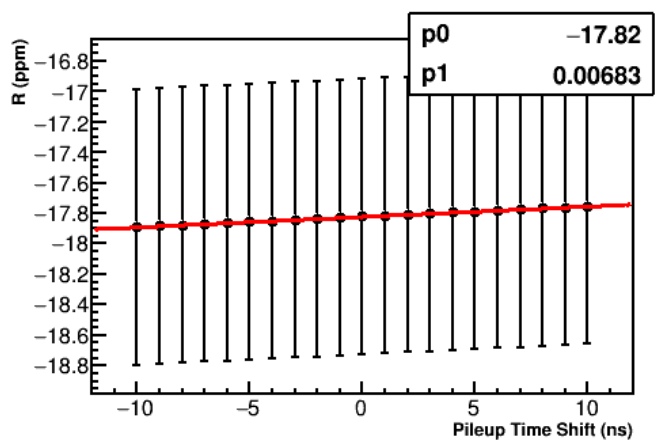

(b) $R$ versus pileup time-shift. The parameter $p_{1}$ gives the sensitivity of $R$ to the value of the pile time-shift, with units in $\mathrm{ppm} / \mathrm{ns}$.

Figure 5·41: Scan over pileup time-shift. Data are from the 9d dataset.

equally well with all pileup phases. Because the width of the $\chi^{2}$ parabolic fit cannot be taken as the uncertainty in the pileup time-shift parameter, the uncertainty is instead taken conservatively at half the ADT at $2.5 \mathrm{~ns}$. The systematic error is calculated in the same way as for the pileup amplitude uncertainty,

$$
\delta R=\sigma_{P_{t}} \times \frac{d R}{d P_{t}}
$$

where $P_{t}$ is the value of the pileup time-shift. The sensitivities of $R$ to the pileup timeshift and corresponding systematic errors for the Run 1 precession frequency analysis datasets are given in Table 5.11. The sensitivities are on the order of $5-8 \mathrm{ppb} / \mathrm{ns}$, with systematic errors ranging from $14-19 \mathrm{ppb}$.

Mis-reconstruction of pileup pulse energies means that pileup shadow doublets will be added or lost near the applied energy threshold, leading to an error in the energy-dependent pileup phase, and by extension a systematic error on $R$. This would happen if the energy sum of two clusters on top of one another is different than two separated by a small time-gap as a result of detector performance. The energy of 


\begin{tabular}{lcc}
\hline \multicolumn{2}{c}{ Systematic Error due to Pileup Time Shift } \\
\hline \hline Dataset & $d R / d P_{t}$ & $\boldsymbol{\delta} \boldsymbol{R}$ \\
\hline $60 \mathrm{~h}$ & 7.0 & $\mathbf{1 7 . 6}$ \\
HighKick & 7.6 & $\mathbf{1 9 . 0}$ \\
9d & 6.8 & $\mathbf{1 7 . 1}$ \\
Endgame & 5.7 & $\mathbf{1 4 . 3}$ \\
\hline
\end{tabular}

Table 5.11: Systematic error due to the pileup time-shift parameter $P_{t}$, in the Ratio Method fits for the Run 1 precession frequency analysis datasets. The bold column gives the systematic errors on $R$. Units for $d R / d P_{t}$ and $\delta R$ are $\mathrm{ppb} / \mathrm{ns}$ and $\mathrm{ppb}$ respectively. As described in the text, the error on $P_{t}$ is taken to be $2.5 \mathrm{~ns}$.

the shadow doublets is dependent on the constant $C$ in the calculation of $E_{\text {doublet }}$ as given in Equation 5.2. In order to calculate the systematic error from the energy construction, the parameter $C$ was scanned from 0.9 to 1.1 , in steps of 0.01 . The results of the study for the $9 \mathrm{~d}$ dataset are shown in Figure $5 \cdot 42$. The systematic error on $R$ is calculated as

$$
\delta R=\sigma_{C} \times \frac{d R}{d C} .
$$

Similarly to the pileup amplitude error, there is a clear minimum in the $\chi^{2}$ results which can be used to estimate the uncertainty in the pileup energy scale. Table 5.12 gives the sensitivities of $R$ to the pileup energy scale, uncertainties in the pileup energy scale, and the corresponding final systematic errors for the Run 1 precession frequency analysis datasets. As shown the uncertainties on the pileup energy scale are of order 1 to $2 \%$, and the value for $C$ which produces the minimum in the $\chi^{2}$ results is consistent with 120 . Interestingly, the sensitivity of $R$ to $C$ in the $60 \mathrm{~h}$ dataset is noticeably larger than in the rest of the datasets; the origin of this is currently under investigation. Regardless, the systematic error is still small at $19.4 \mathrm{ppb}$.

\footnotetext{
${ }^{20}$ Because the spatial separation is turned off in the clustering portion of the reconstruction, a value of $C=1$ is to be expected.
} 


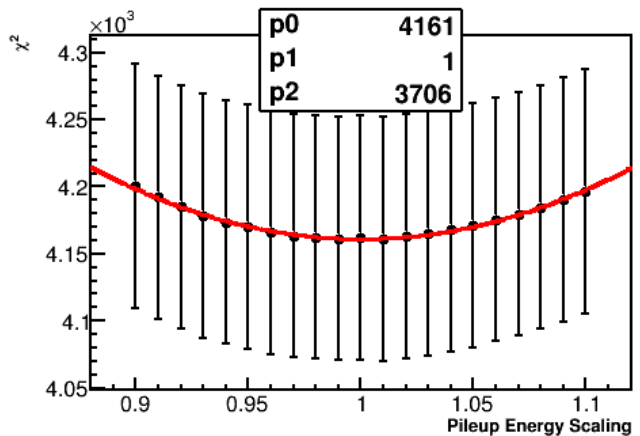

(a) $\chi^{2}$ versus pileup energy scale. The parabolic fit equation used was $y=$ $p_{2}\left(x-p_{1}\right)^{2}+p_{0}$.

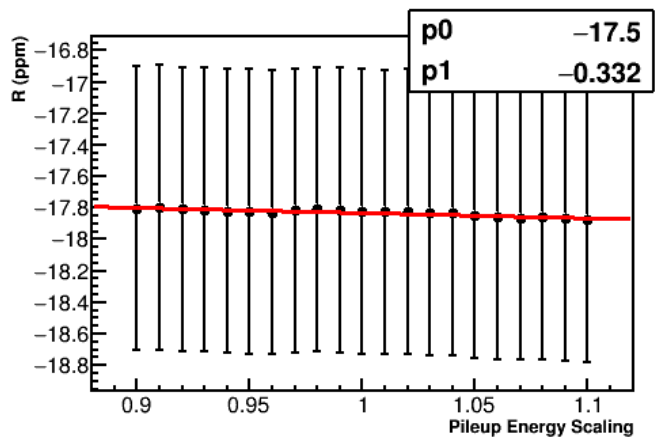

(b) $R$ versus $C$. The parameter $p_{1}$ gives the sensitivity of $R$ to the value of $C$, with units in ppm.

Figure 5.42: Scan over pileup energy scale. Data are from the $9 \mathrm{~d}$ dataset.

\begin{tabular}{lcccc}
\hline \multicolumn{4}{c}{ Systematic Error due to Pileup Energy Scale } \\
\hline \hline Dataset & $d R / d C$ & $\sigma_{C}$ & $C_{\min }$ & $\boldsymbol{\delta R}$ \\
\hline $60 \mathrm{~h}$ & -835.1 & 0.023 & 0.997 & $\mathbf{1 9 . 4}$ \\
HighKick & -167.7 & 0.022 & 0.995 & $\mathbf{3 . 7}$ \\
9d & -332.0 & 0.016 & 1.000 & $\mathbf{5 . 5}$ \\
Endgame & -431.4 & 0.012 & 0.982 & $\mathbf{5 . 3}$ \\
\hline
\end{tabular}

Table 5.12: Systematic error due to the fixed pileup energy scale parameter $C$, in the Ratio Method fits for the Run 1 precession frequency analysis. The bold column gives the systematic errors on $R$. Units for $d R / d C$ and $\delta R$ are in ppb. 


\begin{tabular}{lcccrc}
\hline \multicolumn{7}{c}{ Total Pileup Systematic Errors } \\
\hline \hline Type of Error & Parameter & $60 \mathrm{~h}$ & HighKick & \multicolumn{1}{c}{$9 \mathrm{~d}$} & Endgame \\
\hline Amplitude & $P_{m}$ & 22.2 & 19.0 & 9.0 & 9.4 \\
Phase (time) & $P_{t}$ & 17.6 & 19.0 & 17.1 & 14.3 \\
Phase (energy) & $C$ & 19.4 & 3.7 & 5.5 & 5.3 \\
\hline Quadrature sum & & 34.3 & 27.1 & 20.1 & 17.9 \\
\hline
\end{tabular}

Table 5.13: Total pileup systematic errors for the Run 1 precession frequency analysis datasets.

Table 5.13 gives the quadrature sum for the total pileup systematic errors for the different Run 1 datasets. As shown, for each dataset the total error is below the target final error of $40 \mathrm{ppb}$, despite the contamination in the pileup shadow method. For future runs of the experiment with increased muon storage and therefore increased pileup, these errors may grow. In that case either the pileup shadow method might need to be improved to account for the contamination and pileup triplets, or discarded in favor of a different method.

\subsubsection{Gain systematic errors}

As described in Section 5.1, the energies of the positron hits in the calorimeter crystals are gain-corrected for in-fill, short-term double pulse, and out-of-fill effects. The latter is largely temperature dependent and occurs over time scales much longer than a fill. This does not bias the precession frequency measurement as the phase is not timedependent over the course of a fill. For the cases of in-fill or STDP gain variations, any uncorrected fluctuations in the gain causes an effective change in the energy threshold over the course of a fill, which then modifies the average measured phase of the detected positrons. This causes a systematic shift in the extracted $R$ value.

The IFG function as measured by the laser calibration system, described in Section 3.2.3, relates the measured energies of the individual crystal hits to the true 
energies as a function of time in-fill. It is given by 75

$$
E=E_{0}\left(1-C_{A} e^{-t / \tau_{g}}\right)
$$

where $E_{0}$ is the 'true' energy of the crystal hit, $E$ is the measured energy, and constants $C_{A}$ and $\tau_{g}$ are measured with the laser calibration system. In order to determine a systematic error from the applied IFG function, the IFG function was re-applied to the crystal hits with modified parameters. Specifically, the amplitude of the IFG function $C_{A}$ and the lifetime $\tau_{g}$ were scanned over in multiplicative steps, separately, such that all crystal energies included in a cluster hit were adjusted before re-summing. The multipliers applied to the IFG parameters were scanned from 0 to 2 in steps of 0.25. The results of the scans for the $60 \mathrm{~h}$ dataset are shown in Figure $5 \cdot 43$, with a comparison to the results from fits with the T-Method. It is immediately apparent that the sensitivities of the T-Method to the IFG parameters are significantly greater than that of the Ratio Method. The Ratio Method's insensitivity to smoothly varying effects, which it divides out, is one of it's primary strengths. In fact, while there is an observable minimum in the T-Method $\chi^{2}$ results for the amplitude multiplier scan, no such minimum exists for the Ratio Method results.

Tables 5.14 and 5.15 give the results for the IFG amplitude multiplier and lifetime multiplier scans respectively for the different datasets. Sensitivities for both the TMethod and Ratio Method fits are included. The uncertainties on the IFG parameters from fits to the laser data were in general of order 25\% 121. This uncertainty multiplied against the measured Ratio Method sensitivities results in systematic errors of $<6 \mathrm{ppb}$ on the amplitude multiplier and $<17 \mathrm{ppb}$ for the lifetime multiplier for the various datasets. The individual numbers are given in column 5 of Table 5.14 and column 3 of Table 5.15 respectively. 


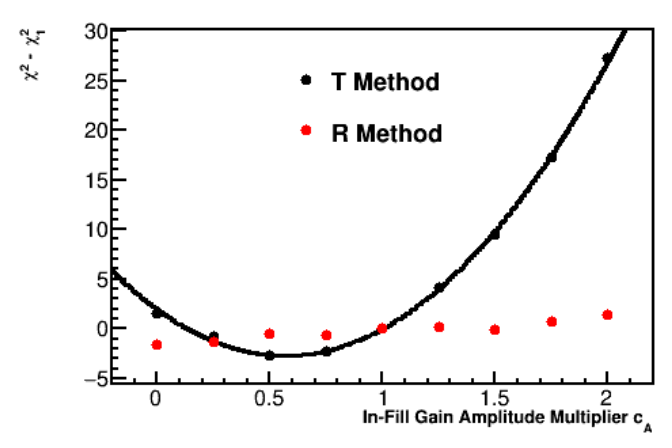

(a) Normalized $\chi^{2}$ vs IFG amplitude multiplier. The T-Method points fit nicely to a parabolic curve while the Ratio Method points do not.

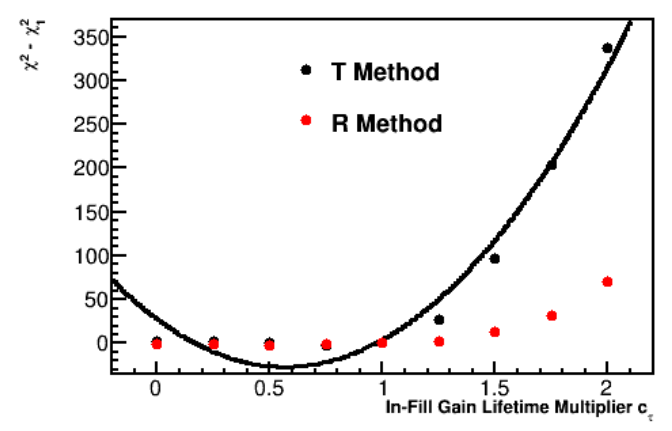

(c) Normalized $\chi^{2}$ vs IFG lifetime multiplier. Both T-Method and R-Method points flatten out at low multipliers, however the rise at high multipliers is larger for the T-Method.

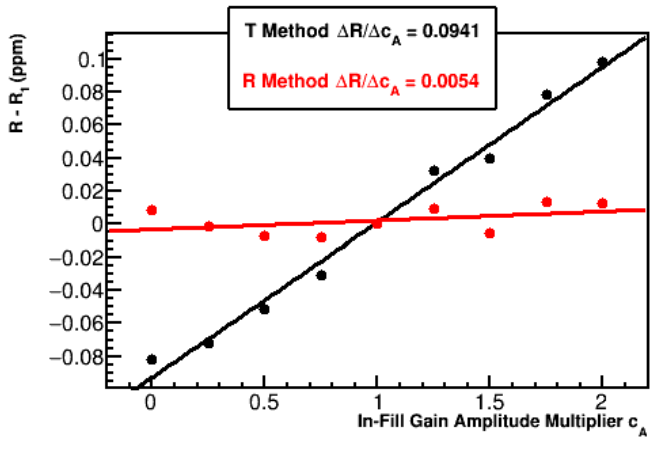

(b) $R$ values vs IFG amplitude multiplier. Both sets of points are fit to straight lines, and the slopes are included in the text box in units of ppm.

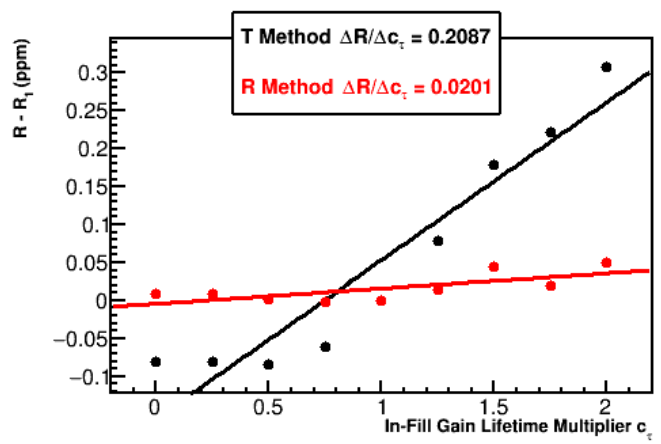

(d) $R$ values vs IFG lifetime multiplier. Both sets of points are fit to straight lines, and the slopes are included in the text box in units of ppm.

Figure 5·43: Fitted $\chi^{2}$ 's and $R$ values for the $60 \mathrm{~h}$ dataset as a function of IFG amplitude multiplier (top) and IFG lifetime multiplier (bottom). Results with ratio fits (red) are compared to T-Method fits (black). Values are normalized to their $C_{A}=1$ and $C_{\tau}=1$ results respectively in order to put the curves on the same scale. As shown the sensitivities in the T-Method to the IFG parameters are significantly larger than that in the Ratio Method. This is one of the primary strengths of the Ratio Method. 


\section{Systematic Error due to IFG Amplitude}

\begin{tabular}{lccccccc}
\hline \hline Dataset & T-Method & T-Method & R-Method & R-Method & R-Method & R-Method & R-Method \\
\hline 60h & $d R / d C_{A}$ & $C_{A_{\min }}$ & $d R / d C_{A}$ & $\boldsymbol{\delta}_{\sigma=0.25}$ & $\Delta R_{0 x}$ & $\Delta R_{2 x}$ & $\boldsymbol{\delta}_{\max -0 \times} / 2 \mathrm{x}$ \\
HighKick & 94.1 & 0.57 & 5.4 & $\mathbf{1 . 4}$ & 8.2 & 12.4 & - \\
9d & 69.5 & 0.70 & -23.6 & $\mathbf{5 . 9}$ & 28.6 & -16.0 & $\mathbf{2 8 . 6}$ \\
Endgame & 64.9 & 0.27 & 1.4 & $\mathbf{0 . 4}$ & -2.5 & -3.9 & - \\
\hline
\end{tabular}

Table 5.14: Sensitivities and systematic errors for the IFG amplitude. T-Method sensitivities are included for comparison, along with the $\chi^{2}$ minima. Also included are changes in $R$ for fits with IFG amplitude multipliers of $0 \mathrm{x}$ and $2 \mathrm{x}$. Systematic error columns are in bold, where the one on the left corresponds to the Ratio Method sensitivity multiplied by a $25 \%$ uncertainty in the amplitude, and the one on the right corresponds to the absolute value of the maximum change in $R$ with the $0 \mathrm{x}$ and $2 \mathrm{x}$ multipliers applied. Only the HighKick and Endgame values are used from the column on the far right. Units for errors and sensitivities are in ppb. 


\begin{tabular}{|c|c|c|c|c|}
\hline \multicolumn{5}{|c|}{ Systematic Error due to IFG Lifetime } \\
\hline & T-Method & R-Method & R-Method & R-Method \\
\hline Dataset & $d R / d C_{\tau}$ & $d R / d C_{\tau}$ & $\boldsymbol{\delta} \boldsymbol{R}_{\sigma=0.25}$ & $\Delta R_{0 x}$ \\
\hline $60 \mathrm{~h}$ & 208.7 & 20.1 & 5.0 & 8.2 \\
\hline HighKick & 114.7 & -44.8 & 11.2 & 28.6 \\
\hline $9 \mathrm{~d}$ & 81.7 & -46.4 & 11.6 & -2.5 \\
\hline Endgame & 207.3 & 66.1 & 16.5 & -6.9 \\
\hline
\end{tabular}

Table 5.15: Sensitivities and systematic errors for the IFG lifetime. TMethod sensitivities are included for comparison. The systematic error columns is in bold, where the error corresponds to the Ratio Method sensitivity multiplied by a $25 \%$ uncertainty in the lifetime. Units for errors and sensitivities are in ppb.

Examining Table 5.14 in more detail however, some interesting features can be seen. The T-Method results, while not the primary subject of this analysis, nevertheless vary between the datasets, in that the scan minima are not consistent with one another. For the Ratio Method results, the HighKick and Endgame datasets exhibit greater sensitivities to the IFG amplitude than the $60 \mathrm{~h}$ and $9 \mathrm{~d}$ datasets. The HighKick dataset can be seen to have a negative sensitivity compared to the positive sensitivities of the other datasets (and the T-Method sensitivities for all datasets). The Endgame dataset has a greater sensitivity stemming from the fact that fits to the multiplicative factors of $C_{A}=1.75$ and $C_{A}=2$ produced noticeably better $\chi^{2}$ 's and higher $R$ values, $\mathcal{O}(40 \mathrm{ppb})$. These fits pulled the slope up from what is otherwise a very flat fit to the rest of the points.

These various peculiarities imply there are imperfections in the gain corrections which have not been fully accounted for. Indeed recent investigations into the applied gain corrections show that for the HighKick and Endgame datasets, the out-of-fill corrections were improperly applied during data production 122. While this does not introduce a systematic uncertainty as a function of time in-fill as described before, it does shift the scale of the correction and by extension the extracted $R$ value. In 
order to determine more conservative bounds on the systematic errors to account for these deficiencies, the absolute changes in $R$ for the $0 \mathrm{x}$ and $2 \mathrm{x}$ multiplier fits were examined. These values are included in Table 5.14. The maximum of the two values was then taken as the systematic error on $R$ due to the IFG amplitude. In general the systematic errors as calculated from the maximum of the $0 \mathrm{x}$ or $2 \mathrm{x}$ fits are an order of magnitude larger than those errors calculated directly from the sensitivities. Because the scale of these numbers is still small compared to the Run 1 statistical errors however, they are deemed acceptable for this analysis.

As for the lifetime multiplier scan results, it was observed that the $R$ and $\chi^{2}$ plots flattened out for small lifetimes as compared to large lifetimes, with no identifiable minima in the scan results. $R$ was seen to change by very little when the lifetime was small, as shown in the far right column of Table 5.15. Because of the larger changes in $R$ with the larger lifetimes, the sensitivities in the table were pulled to greater values than they otherwise might be. The choice was made to keep these large sensitivities in and use them in the calculation of the systematic error for a conservative approach, again justified by the gain issues mentioned previously.

In the calculation of the combined systematic error due to the IFG function parameters, the amplitude and lifetime parameter errors are added in quadrature, conservatively ignoring correlations between the two parameters in the fits to the laser data. Because the two parameters are correlated to a large degree, the $0 \mathrm{x}$ and $2 \mathrm{x}$ lifetime numbers were not used in the calculation of the HighKick and Endgame lifetime systematic errors as they have already been conservatively treated in the amplitude part of the error ${ }^{21}$. The confidence in the scale of the Ratio Method results is ultimately preserved from the fact that the Ratio Method fits are not as sensitive to the gain variations as the T-Method fits, as the gain effects divide out. Going forward towards the end of the Run 1 analysis, once the outstanding gain issues have been

\footnotetext{
${ }^{21}$ If the amplitude is zero then the lifetime is meaningless, and vice versa.
} 
resolved, the systematic errors should be calculated directly from the sensitivities in all cases.

The STDP correction is similar in many respects to the IFG correction [74]. This correction is applied to pulses close in time, $\mathcal{O}(\mathrm{ns})$, and is applied to the energies of the pulses before the in-fill gain correction. Because of how the time-randomization is applied to the clusters in this analysis, a new version of the same dataset by default uses a different default randomization with respect to the cluster times and the ratio histogram filling. As shown in Section 5.4.4, the width in the fitted $R$ values for many random seeds is of order $\mathcal{O}(100 \mathrm{ppb})$, making direct ratio fit comparisons between the two datasets un-informative. In order to avoid this difficulty and evaluate the systematic error on $R$ due to the application of the STDP correction, a slightly different procedure was undertaken. First, a new production of the $60 \mathrm{~h}$ dataset was processed without the STDP correction applied, and where cluster times were randomized perfill rather than per-cluster, and then fits were then done with the T-Method. Since the per-fill randomization uses fill ID's as the seeds for the randomization, and because the T-Method does not split the data into sub-datasets as the Ratio Method does, the randomization between the $60 \mathrm{~h}$ dataset with and without the STDP correction is identical. The change in $R$ for T-Method fits with and without this correction is then taken as the upper bound on the systematic error for the inclusion of the STDP in the Ratio Method fits. This is reasonable as the previous IFG systematic studies showed a reduction in sensitivity to gain effects which is reasonably extended to the STDP correction error. Table 5.16 gives the T-Method fit results with and without the STDP, along with the change in $R$. This difference was found to be $11.0 \mathrm{ppb}$, which is then taken as the systematic error for all datasets.

Table 5.17 gives the quadrature sum for the total gain systematic errors for the Run 1 precession frequency analysis datasets. As shown for the 60h and 9d datasets, 
Systematic Error due to STDP

\begin{tabular}{lccc}
\hline \hline Fit Type & $R$ with STDP $(\mathrm{ppm})$ & $R$ without STDP $(\mathrm{ppm})$ & $\boldsymbol{\delta} \boldsymbol{R}(\mathrm{ppb})$ \\
\hline T-Method & -20.1619 & -20.1729 & $\mathbf{1 1 . 0}$ \\
\hline
\end{tabular}

Table 5.16: T-Method fit results with and without the STDP gain correction on the $60 \mathrm{~h}$ dataset. T-Method fits were done instead of Ratio Method fits in order to force the cluster-time randomization to be consistent between the two dataset productions. The change in $R$ in the bold column is taken as the upper bound on the systematic error in the Ratio Method due to the STDP gain correction.

\begin{tabular}{lcccc}
\hline \multicolumn{5}{c}{ Total Gain Systematic Errors } \\
\hline \hline Type of Error & $60 \mathrm{~h}$ & HighKick & $9 \mathrm{~d}$ & Endgame \\
\hline IFG amplitude & 1.4 & 28.6 & 0.4 & 43.6 \\
IFG lifetime & 5.0 & 11.2 & 11.6 & 16.5 \\
STDP & 11.0 & 11.0 & 11.0 & 11.0 \\
\hline Quadrature sum & 12.2 & 32.6 & 16.0 & 47.9 \\
\hline
\end{tabular}

Table 5.17: Total gain-related systematic errors for the Run 1 precession frequency analysis datasets. Units are in ppb.

the total error is below the target final error of $20 \mathrm{ppb}$. For the HighKick and Endgame datasets, the errors are slightly larger than $20 \mathrm{ppb}$, and should reduce once their known issues have been resolved. All gain errors will be re-evaluated once the final datasets are available. It is expected that the errors given here are reasonable approximations of the final errors, especially due to the Ratio Method's insensitivity to the gain relative to the T-Method. As a reminder, the errors given here, even with the crude approximations, are small compared to the statistical errors of the various datasets.

\subsubsection{CBO systematic errors}

If the $\mathrm{CBO}$ is mis-modeled then there will be a systematic error on $R$ since there is a an early-to-late change in both the frequency and the scale of the CBO. The 


\begin{tabular}{lc}
\hline \multicolumn{1}{c}{ Change in $\boldsymbol{R}$ with station $\mathbf{1 8}$ CBO parameters } \\
\hline \hline Dataset & $\boldsymbol{\delta} \boldsymbol{R}$ \\
\hline $60 \mathrm{~h}$ & 7.5 \\
HighKick & 0.4 \\
9d & -2.0 \\
Endgame & 8.0 \\
\hline
\end{tabular}

Table 5.18: Changes in the fitted $R$ values with tracker station 18 CBO frequency model parameters, instead of tracker station 12 . The systematic errors are conservatively taken as the absolute values of the changes in $R$. Units are in ppb.

CBO model is well constrained by tracker measurements, but systematic errors can be evaluated by modifying the fixed frequency function given in Equation 5.22, and the decoherence envelope of the CBO.

Table 5.2 gives the CBO frequency model parameters for both tracker stations. The station 12 values are by default used in all fits to the data. Fits were performed with the station 18 values for the various datasets, and the changes in $R$ are given in Table 5.18. While the CBO parameters in the tracking analysis fits do have errors on the parameters, they are tiny compared to the systematic errors between the two tracker stations [113]. Some fits were made by varying the fixed frequency parameters by $1 \sigma$ in their individual respective errors, and the changes in $R$ were found to be negligible. For this reason, the absolute values of the changes in $R$ when using station 12 parameters versus station 18 parameters are conservatively taken as the systematic errors, due to the choice of fixed CBO frequency model parameters.

The shape of the $\mathrm{CBO}$, or the decoherence envelope, is also similarly constrained by the tracking analysis. The envelope is by default taken to be an exponential as given in Equation 5.19, and shown in Figure 4.28. The other envelope which could reasonably exist in the data, from inspection in the tracking analysis, is an exponential 
plus a constant,

$$
e^{-t / \tau_{c b o}} \rightarrow e^{-t / \tau_{c b o}}+C,
$$

where $C$ is some constant $\mathrm{CBO}$ amplitude which persists over the course of the fill. In order to assess this systematic error, this new envelope was introduced into the $N_{c b o}(t)$ fit term. Fits were done with $C$ floating, where the starting values for $C$ in the Ratio Method fits were taken from T-Method fits to the dat222, While in T-Method fits the $C$ parameter converged to values with errors about half the value, in the Ratio Method fits the $C$ parameters had relatively large errors. In spite of the large errors however, the fits converged properly with the floating $C$ parameter. In general the final fit parameters were largely the same, with the exception being the fitted CBO lifetime and amplitude on the $N_{c b o}(t)$ term, where both approximately halved. This is unsurprising as the lifetime is highly correlated to the amplitude. Only in the 9d dataset did some small complications arise, where the fit didn't particularly handle the floating $C$ parameter well. Fixing the lifetime to a value of 99 us, chosen such that the lifetime reduction was somewhat comparable to that seen in the other datasets, the $C$ parameter converged to a value close to and consistent with zero. The fitted values for the $C$ constants and the changes in the final fitted $R$ values are given in Table 5.19, As shown the changes in $R$ are of order 10s of ppb for some of the datasets, with $R$ varying both positively and negatively. The absolute value of these changes in $R$ are conservatively taken as the systematic errors on $R$ for the different datasets.

Table 5.20 gives the quadrature sum for the total $\mathrm{CBO}$ systematic errors for the Run 1 precession frequency analysis datasets. As shown, for each dataset the total error is below the target final error of $30 \mathrm{ppb}$. The changing frequency of the CBO

\footnotetext{
${ }^{22}$ T-Method starting values for $C$ were taken to be 0 which resulted in well-converging fits.
} 


\begin{tabular}{lccr}
\hline \multicolumn{3}{c}{ Systematic Error due to CBO Envelope } \\
\hline \hline Dataset & $C \times 10^{-4}$ & $\sigma_{C} \times 10^{-4}$ & $\boldsymbol{\delta} \boldsymbol{R}$ \\
\hline 60h & 10.7 & 8.3 & 17.6 \\
HighKick & 11.6 & 10.4 & -18.0 \\
9d & -2.1 & 11.1 & 9.3 \\
Endgame & 10.7 & 4.0 & -4.3 \\
\hline
\end{tabular}

Table 5.19: Systematic error on $R$ due to the choice of CBO envelope. The fitted floating parameter $C$ and its error are given along with the change in $R$, compared to the standard exponential envelope in units of ppb.

\begin{tabular}{lcccc}
\hline \multicolumn{5}{c}{ Total CBO Systematic Errors } \\
\hline \hline Type of Error & $60 \mathrm{~h}$ & HighKick & $9 \mathrm{~d}$ & Endgame \\
\hline CBO Frequency Model & 7.5 & 0.4 & 2.0 & 8.0 \\
CBO Decoherence Envelope & 17.6 & 18.0 & 9.3 & 4.3 \\
\hline Quadrature sum & 19.1 & 18.0 & 9.5 & 9.1 \\
\hline
\end{tabular}

Table 5.20: Total CBO-related systematic errors for the Run 1 precession frequency analysis datasets.

in Run 1 was eliminated in Run 2, implying a reduction in any associated systematic errors. The increased statistics combined with the tracking analysis should constrain measurements of the $\mathrm{CBO}$ decoherence envelope even further, similarly reducing any associated systematic errors. At the same time, increased statistics might bring out higher order $\mathrm{CBO}$ effects which require improved modeling.

\subsubsection{Lost muon systematic errors}

There are two types of systematic errors arising from lost muons. The first is due to mis-construction of the shape of the lost muon spectrum $L(t)$ included in the fits, and the second is due to any $g-2$ phase difference between the stored and lost muon populations.

As mentioned in Section 5.3.3, the triples spectrum is made with cuts as defined 


\begin{tabular}{lcccc}
\hline \multicolumn{4}{c}{$\Delta \boldsymbol{R}$ with Various Lost Muon Cuts } \\
\hline \hline \multicolumn{4}{c}{$9 \mathrm{~d}$ Dataset } & \multicolumn{2}{c}{ Endgame Dataset } \\
\hline Type of fit or cut & $\Delta R(\mathrm{ppb})$ & $\kappa_{\text {loss }}$ & $\Delta R(\mathrm{ppb})$ & $\kappa_{\text {loss }}$ \\
\hline Default cuts & 0 & 2.510 & 0 & 2.345 \\
No quadruple subtraction & 0.2 & 1.811 & -0.1 & 1.717 \\
No accidental subtraction & 0.1 & 2.503 & $<0.1$ & 2.339 \\
$5 \mathrm{~ns} \leq \Delta t_{12,23} \leq 8.5 \mathrm{~ns}$ & 0.1 & 2.507 & $<0.1$ & 1.709 \\
$100 \mathrm{MeV} \leq E_{1,2,3} \leq 500 \mathrm{MeV}$ & $<0.1$ & 2.253 & $<0.1$ & 2.090 \\
$\Delta t_{13} \leq 12.5 \mathrm{~ns}$ & 0.1 & 4.469 & -0.3 & 4.248 \\
\hline
\end{tabular}

Table 5.21: Effect on the fitted $R$ value in the 9d and Endgame datasets with various cuts used or backgrounds subtracted. Also included are the various corresponding $\kappa_{\text {loss }}$ values which compensate for the level of statistics contained within $L(t)$ due to the differing cuts.

in Table 5.4. Various backgrounds are subtracted off the triples spectrum in order to generate a clean sample of lost muons. Table 5.21 gives the changes in $R$ for various sets of cuts and background subtractions for the $9 \mathrm{~d}$ and Endgame datasets. As shown, the various different backgrounds and cuts ultimately make very little difference in the final fitted $R$ values. Similarly, stable beam contaminants in the form of deuterons and protons contaminate the lost muon spectrum. The former are largely removed by straightforward $\Delta t_{12}$ and $\Delta t_{13}$ cuts. The latter can be mostly removed by cutting on the negative side of the $\Delta t_{13}$ distribution, with $\Delta t_{13} \leq 12.5 \mathrm{~ns}$ which separates the populations more readily, shown in Figure 5.44. While this does largely remove the protons at the cost of statistics, the fitted $\kappa_{\text {loss }}$ parameter simply grows larger to compensate. Ultimately the effect on $R$ is still negligible, with $\Delta R=-0.3 \mathrm{ppb}$ for the Endgame dataset. The sum of these separate types of errors is conservatively taken at $0.5 \mathrm{ppb}$ for all datasets 23 .

Because $\kappa_{\text {loss }}$ is fixed in the Ratio Method fits from corresponding T-Method fits,

\footnotetext{
${ }^{23}$ For the T-Method fits, though the changes in $R$ are noticeably larger with the various cuts, they are still the same order of magnitude and the error is conservatively below $1 \mathrm{ppb}$.
} 


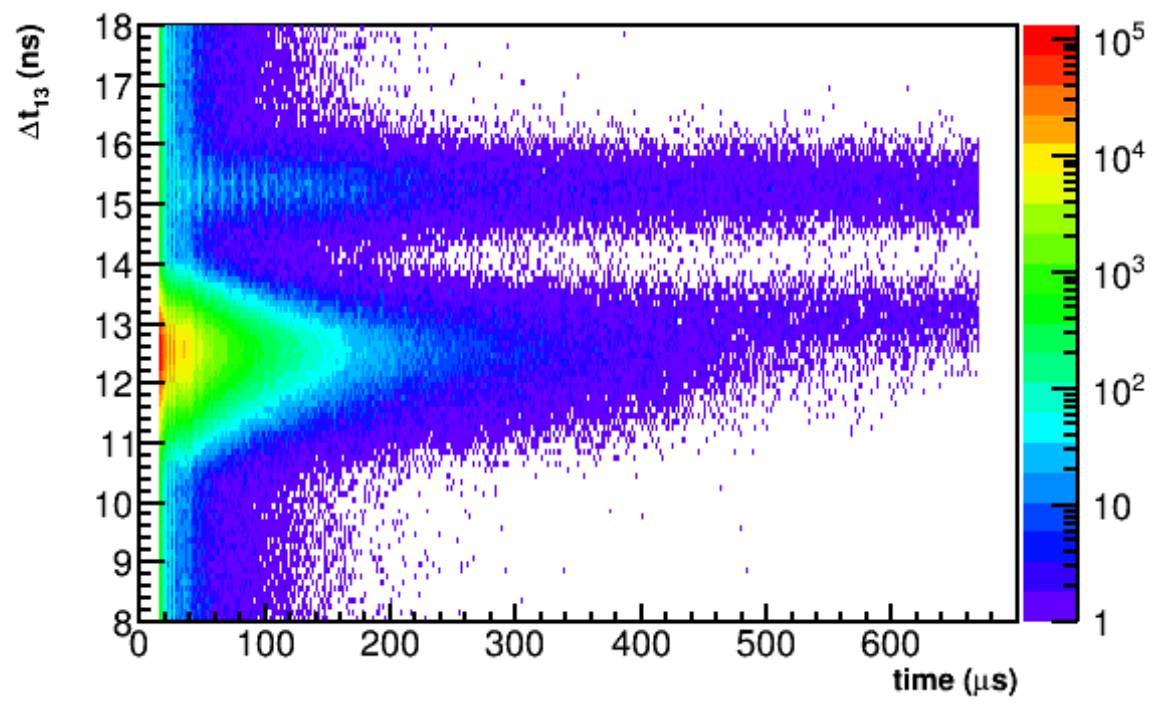

Figure 5.44: Lost muon $\Delta t_{13}$ distribution as a function of time infill. The band of hits centered just above $15 \mathrm{~ns}$ corresponds to the deuteron contamination, while the band of hits centered just above $13 \mathrm{~ns}$ corresponds to the proton contamination.

the statistical correlation to $R$ is neglected and a systematic error must be estimated. The systematic error can be determined by scanning over the value of $\kappa_{\text {loss }}$ as in Figure $5 \cdot 45$. The uncertainty in $\kappa_{\text {loss }}$ is taken as the statistical error determined in the T-Method fit to the same data. Table 5.22 gives the systematic errors on $R$ for the various datasets. As shown the systematic errors are all small, $\delta R<5 \mathrm{ppb}$. Also shown are the changes in $R$ with the muon loss term in the fit versus without it entirely. The scale of the changes is on the order of $10 \mathrm{~s}$ of ppb, hence the decision to include the lost muon term in the fit, though it could reasonably be excluded as long as the larger error is applied.

The most dangerous systematic error from lost muons is due to the $g-2$ phase of the lost muon population. If muon losses originate from a population of muons with a different average phase than the stored muons, then there will be a phase shift over the course of a fill, and thus a systematic error. Simulation and data have shown a correlation between $g-2$ phase and muon momentum at injection, with the 


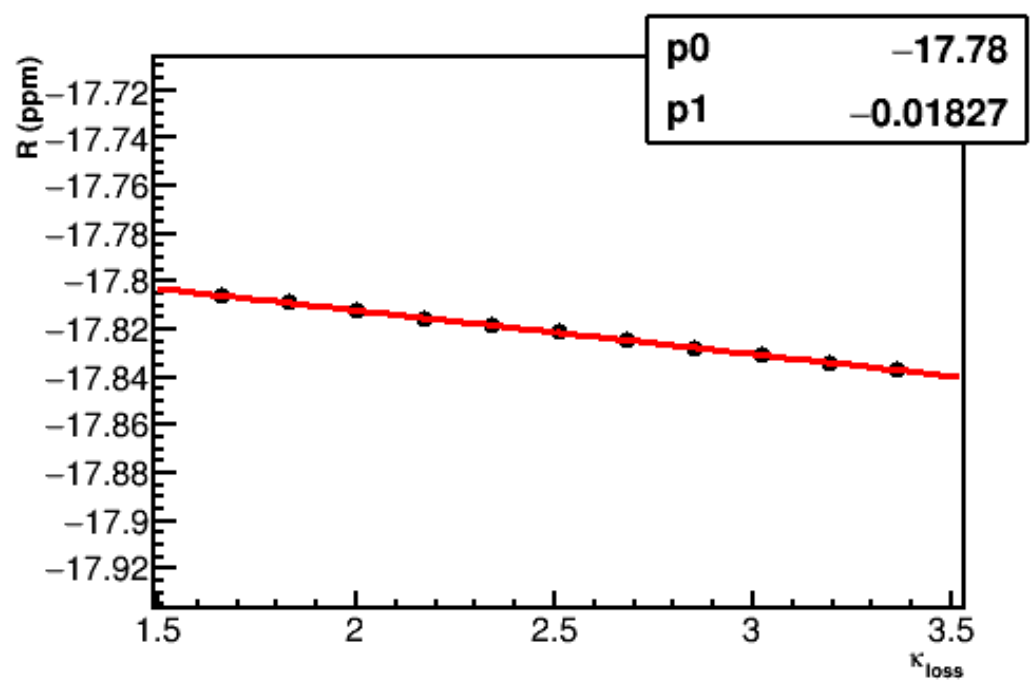

Figure 5.45: The sensitivity of $R$ to the fixed $\kappa_{\text {loss }}$ parameter. Error bars have been removed from the plot in order to show the trend more clearly. Units are in ppm, data are from the 9d dataset.

\begin{tabular}{lcccc}
\hline \multicolumn{4}{c}{ Systematic Error due to Fixed $\boldsymbol{\kappa}_{\text {loss }}$} \\
\hline \hline Dataset & $d R / d \kappa_{\text {loss }}$ & $\sigma_{\kappa_{\text {loss }}}$ & $\boldsymbol{\delta} \boldsymbol{R}$ & $\Delta R_{(\mathrm{w} / \mathrm{w} / \mathrm{o})}$ \\
\hline 60h & -3.5 & 0.338 & $\mathbf{1 . 2}$ & -31.4 \\
HighKick & -7.1 & 0.697 & $\mathbf{4 . 9}$ & -40.1 \\
9d & -18.3 & 0.170 & $\mathbf{3 . 1}$ & -45.7 \\
Endgame & -2.6 & 0.038 & $\mathbf{0 . 1}$ & -6.1 \\
\hline
\end{tabular}

Table 5.22: Systematic error due to the fixed $\kappa_{\text {loss }}$ parameter in the Ratio Method fits for the Run 1 precession frequency analysis datasets. All units are in ppb except for the $\sigma_{\kappa_{\text {los }}}$ parameter which is unit-less. $\sigma_{\kappa_{\text {loss }}}$ comes from the T-Method fit results and scales with the number of statistics. The bold column gives the systematic errors on $R$. The far right column gives the change in $R$ with the $\kappa_{\text {loss }}$ parameter in the fits versus without it entirely. 
data driven study having returned a correlation of $10.3 \pm 0.1 \mathrm{mrad} / \%(\Delta p / p)[123]$. The phase-momentum correlation originates from the experience of muons within the accelerator beam-line before injection. One simple explanation is that higher momentum muons take longer path lengths inside the accelerator's steering magnetic fields, during which their spins will precess and their $g-2$ phases change. If the losses are momentum dependent and the phase-momentum correlation is preserved after injection, then there will be a systematic error. Losses are expected to be momentum dependent [124]; the low kick applied in Run 1 resulted in an off-center stored muon beam such that there were more high momentum muons at greater radii near the outside edge of the storage aperture, than low momentum muons on the inside of the storage region. The high momentum muons at the outside edge of the storage region have a higher chance of impacting material and getting lost, as they undergo their individual betatron oscillations. Further simulation efforts and data-driven studies are underway to understand and quantify the phase-momentum correlation and momentum-dependent loss probabilities. Here is given an estimation of the muon loss phase bias systematic error using the measured losses for the different Run 1 datasets.

From Equation 5.34, the systematic shift in $\omega_{a}$ can be written as

$$
\frac{\Delta \omega_{a}}{\omega_{a}}=\frac{1}{\omega_{a}} \frac{d\langle\phi\rangle}{d t}
$$

where $d\langle\phi\rangle / d t$ is the change in average phase over time. With some assumptions made with regards to 'core' and 'edge' muon populations, where lost muons can only originate from the latter, this can be written as 124

$$
\frac{d\langle\phi\rangle}{d t}=f_{c} \cdot d f_{l} \cdot \Delta \phi_{c-e}
$$

where $f_{c}$ is the fraction of muons in the core population to all muons, $d f_{l}$ is the 


\begin{tabular}{lcc}
\hline \multicolumn{2}{c}{ Systematic Error due to Lost Muon Phase Bias } \\
\hline \hline Dataset & $f_{l}(\% / 70 \mu \mathrm{s})$ & $\Delta \omega_{a} / \omega_{a}(\mathrm{ppb})$ \\
\hline $60 \mathrm{~h}$ & 0.60 & -53.6 \\
HighKick & 0.20 & -17.9 \\
$9 \mathrm{~d}$ & 0.25 & -22.3 \\
Endgame & 0.65 & -58.0 \\
\hline
\end{tabular}

Table 5.23: Loss rates and associated shift in $\omega_{a}$ for the Run 1 precession frequency analysis datasets. Loss rates are determined by inspection of the respective curves for the different datasets, and are approximate. The systematic shifts here are negative, due to the fact that the average phase of the stored muons is greater than that of the lost muons, as determined in simulation.

fractional loss rate, and $\Delta \phi_{c-e}$ is the average phase difference between the core and edge muon populations. The simulation studies in the afore-referenced document gives estimates of $f_{c} \approx 0.9$ and $\Delta \phi_{c-e} \approx 1 \mathrm{mrad}$. The fractional losses, the integral in Equation 5.30 times the final fitted $\kappa_{\text {loss }}$ parameter, assuming no losses are included in $L(t)$ before the fit start time, for the Run 1 precession frequency analysis datasets as determined in this analysis are shown in Figure 5.46. As shown the losses rise sharply at early times before leveling off to a constant rate around $100 \mu \mathrm{s}$ into the fill. Taking the first $70 \mu \mathrm{s}$ of the fit and assuming that this loss rate is constant over the course of the fill, the approximate loss rates for the different datasets can be determined Applying the various quantities into Equation 5.42, along with $\omega_{a} \approx 1.44 \mathrm{rad} / \mu \mathrm{s}$, the systematic shifts in the precession frequency are found. The loss rates and systematic shifts for the various datasets are given in Table 5.23 .

Taking the shifts in $\omega_{a}$ as the systematic errors, the approximations here are on the order of $50-60 \mathrm{ppb}$ for the $60 \mathrm{~h}$ and Endgame datasets, and on the order of $20 \mathrm{ppb}$ for the HighKick and 9d datasets. These estimates are the same order of mag-

\footnotetext{
${ }^{24}$ While the loss rate reduces after $100 \mu \mathrm{s}$, losses occur mostly at early times, so this is a fine approximation.
} 


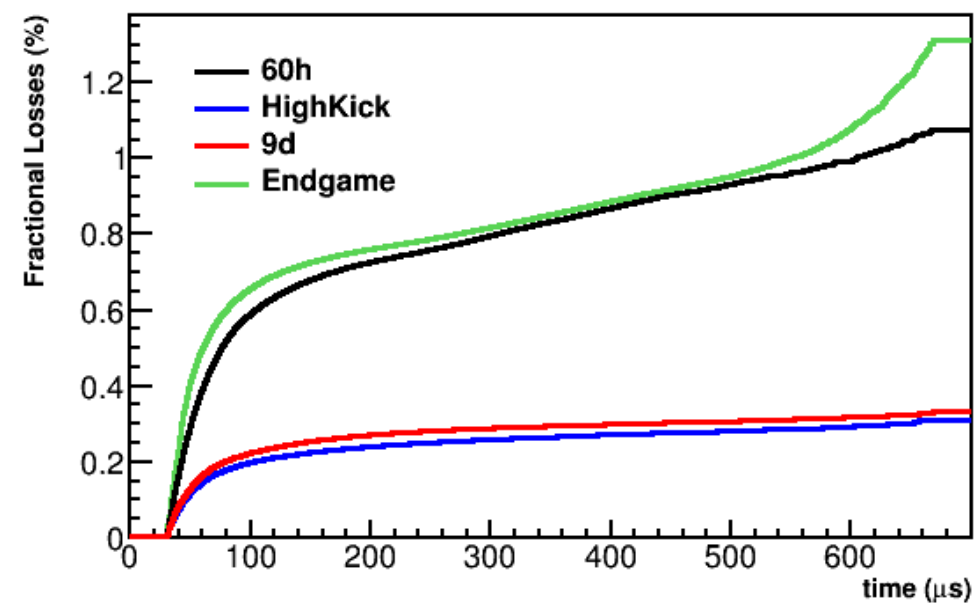

Figure 5.46: Fractional losses for the Run 1 precession frequency analysis datasets. The curves begin at $30.2 \mu$ s and end at $650 \mu \mathrm{s}$, corresponding to the fit range. A value of $1 \%$ at a time $t$ indicates that there are $1 \%$ fewer stored muons at that time than there would be if there were no losses at all. The Endgame and $60 \mathrm{~h}$ datasets have more losses than the $9 \mathrm{~d}$ and HighKick datasets. This is due to the greater kicker voltages used in the latter datasets which put the stored muon beam on a more central orbit. The upward tail at the end of the Endgame dataset corresponds to the remnant proton contamination.

nitude as determined in other evaluations with slightly different parameters [125]. As simulations improve and more studies are undertaken, the uncertainties here may potentially be replaced in favor of actual corrections, in which case the systematic errors would then be the uncertainties in the evaluation of those corrections. Regardless, while greater than the target goal of $20 \mathrm{ppb}$, at least in the cases of the $60 \mathrm{~h}$ and Endgame datasets, the systematic errors listed here are nevertheless small compared to the Run 1 statistical errors. They are expected to decrease in future runs where all datasets have greater kicker voltages as in the HighKick and 9d datasets. 


\subsubsection{Ratio construction systematic errors}

In the construction of the ratio data, when filling the four sub-datasets as in Equation 5.11, the parameters $T_{a}$ and $\tau_{\mu}$ for the $g-2$ period and muon lifetime need to be known a priori. If these parameters are incorrectly chosen, then it is possible there will be a systematic shift on $R$. This is especially important when considering $T_{a}$, because the quantity which the E989 experiment is measuring must be used in the analysis, creating a sort of self-dependence. The question then naturally arises as to how well the $T_{a}$ parameter needs to be known. As described in Section 5.2.2, the input value for $T_{a}$ is nominally taken as the result from the E821 experiment.

In order to determine systematic errors from the choices of $T_{a}$ and $\tau_{\mu}$, they were scanned over when forming the ratio data. The input value for $T_{a}$ was varied from $-30 \mathrm{ppm}$ to $30 \mathrm{ppm}$ in steps of $3 \mathrm{ppm}$, while the input value for $\tau_{\mu}$ was varied from $64.04 \mu \mathrm{s}$ to $64.84 \mu \mathrm{s}$ in steps of $0.04 \mu \mathrm{s}$. See Figure $5 \cdot 47$ for scan results for the $60 \mathrm{~h}$ dataset. See Table 5.24 for the sensitivities determined from the scans for all datasets. As shown the sensitivities vary both positively and negatively for the different datasets, and are extremely small. The positive and negative variations imply there is no real systematic effect at play, and that as long as a reasonable choice for these two parameters is made, then the ratio data are very insensitive to the exact values chosen.

In order to be conservative however, a scale for the changes in $R$ is given. Since the measured $g-2$ period in the data is already modified by the hardware blinding, it is technically the hardware shifted $g-2$ period that should be used. The calorimeter digitizers use a $40+\delta \mathrm{MHz}$ clock which has been blinded to a value in the range of 39.997 to $39.999 \mathrm{MHz} 111$. This corresponds to a $75 \mathrm{ppm}$ range in the frequency, in a uniform distribution. Calculating the uncertainty from the uniform distribution and adding it in quadrature with a conservative $1 \mathrm{ppm}$ uncertainty in the guess on the 


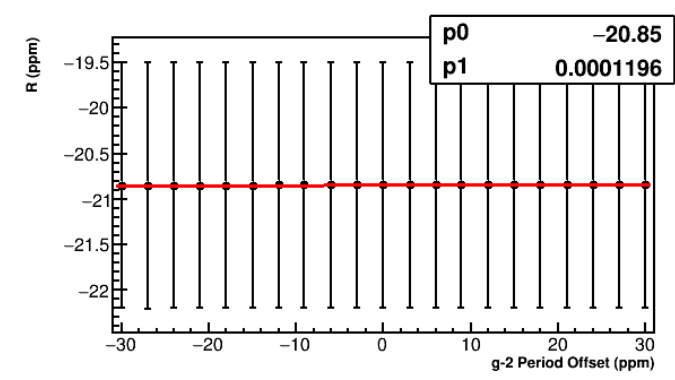

(a) $R$ versus input value for $T_{a}$, where the $\mathrm{x}$-axis is given in units of a ppm level shift of the default choice for $T_{a}$. Parameter $p_{1}$ gives the sensitivity in $\mathrm{ppm} / \mathrm{ppm}$.

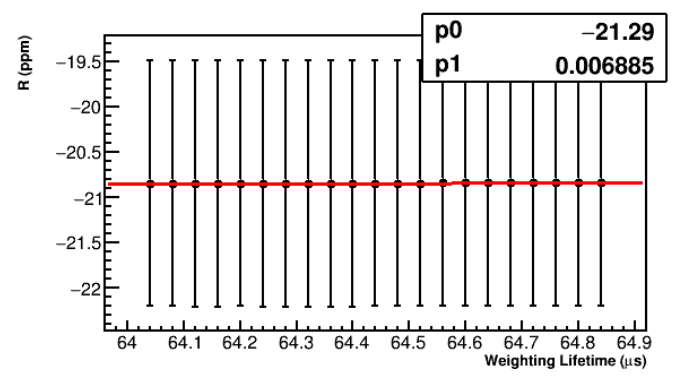

(b) $R$ versus the input value for $\tau_{\mu}$. Parameter $p_{1}$ gives the sensitivity in $\mathrm{ppm} / \mathrm{\mu s}$.

Figure 5.47: Scans over ratio construction parameters for the $60 \mathrm{~h}$ dataset. In general the points are randomly spread around, and sensitivities are practically negligible.

\begin{tabular}{lcc}
\hline \multicolumn{2}{c}{ Sensitivity to Ratio Construction } & Parameters \\
\hline \hline Dataset & $d R / d_{T_{a}}$ & $d R / d_{\tau_{\mu}}$ \\
\hline 60h & 0.1 & 6.9 \\
HighKick & -0.1 & -4.1 \\
9d & $<0.1$ & -1.1 \\
Endgame & $<0.1$ & 0.6 \\
\hline
\end{tabular}

Table 5.24: Sensitivities of $R$ to ratio construction parameters. $d R / d_{T_{a}}$ is in units of $\mathrm{ppb} / \mathrm{ppm}$, while $d R / d_{\tau_{\mu}}$ is in units of $\mathrm{ppb} / \mu \mathrm{s}$. In both cases the sensitivities are both extremely small, and vary negatively and positively for the different datasets. 
true $g-2$ period from the E821 result,

$$
\delta T_{a}=\sqrt{(75)^{2} / 12+1^{2}}=21.7 \mathrm{ppm}
$$

This results in a change in $R$ on the order of $2.2 \mathrm{ppb}$ for the $60 \mathrm{~h}$ and HighKick datasets, and less for the $9 \mathrm{~d}$ and Endgame datasets. $2.2 \mathrm{ppb}$ is therefore taken as the systematic error for all datasets due to the choice of $T_{a}$.

Similarly, the sensitivities of $R$ to the chosen muon lifetime are very small, order $\mathrm{ppb} / \mathrm{\mu s}$. Since the uncertainties in the muon lifetime are of order ns from fits to the data, any systematic errors from this parameter would be completely negligible even if the sensitivities all had the same sign. For purposes of this analysis, the systematic errors are therefore all taken as $<0.1 \mathrm{ppb}$ due to the choice of $\tau_{\mu}$.

\subsubsection{Binning systematic errors}

When constructing the time spectra, the bin width and the starting edge of the bin are by default chosen to be $149.2 \mathrm{~ns}$ and 0 ns respectively. In order to calculate systematic errors from the choices of these parameters, the values were scanned over. The bin width was scanned from $148.7 \mathrm{~ns}$ to $149.7 \mathrm{~ns}$ in steps of $0.1 \mathrm{~ns}$, while the bin edge was scanned from $0 \mathrm{~ns}$ to $149.2 \mathrm{~ns}$ in steps of $14.92 \mathrm{~ns}$. Figure $5 \cdot 48$ shows scan results for the 9d dataset. While trends appeared largely random, a straight line was fit to the scan results in order determine the sensitivities of $R$ to both parameters; see Table 5.25. For the bin edge scan, it was verified that a shift of one bin width returned the same fit results as the default shift of $0 \mathrm{~ns}$. This combined with the negligible sensitivities and varying points implies negligible systematic effects on $R$ from the choice of bin edge. For the systematic error due to the choice of bin width, it should be noted that in general the choice of bin width should be optimized to be equal to the peak of the cyclotron period distribution of the stored muons, which from the 


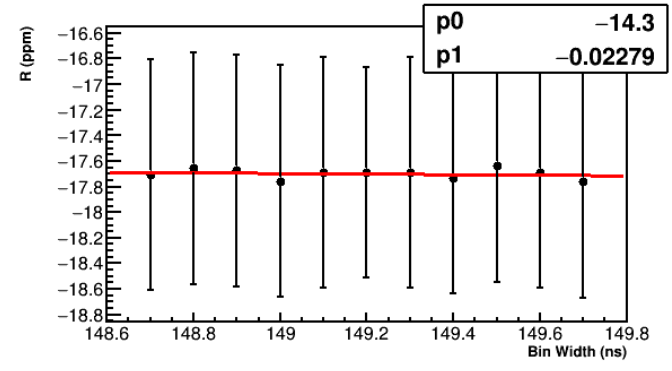

(a) Scan over bin width.

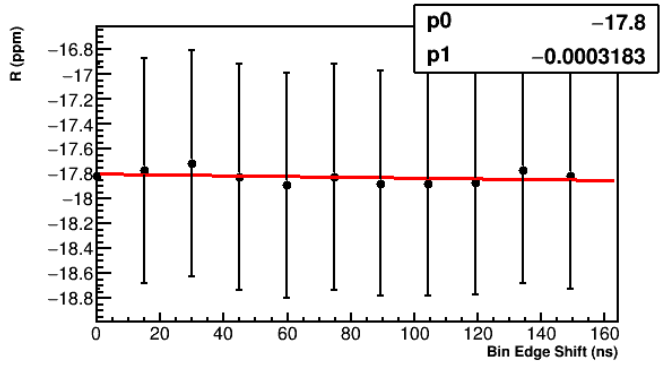

(b) Scan over bin edge.

Figure 5.48: Scans over binning parameters for the 9d dataset. In general the points are randomly spread around, indicating no real systematic effect.

\begin{tabular}{lcc}
\hline \multicolumn{2}{c}{ Sensitivity to Binning Parameters } \\
\hline \hline Dataset & $d R / d_{\text {bin width }}$ & $d R / d_{\text {bin edge }}$ \\
\hline $60 \mathrm{~h}$ & 24.5 & -0.1 \\
HighKick & 6.0 & -0.7 \\
$9 \mathrm{~d}$ & -22.8 & -0.3 \\
Endgame & -41.7 & -0.6 \\
\hline
\end{tabular}

Table 5.25: Sensitivities of $R$ to binning parameters. Units are in $\mathrm{ppb} / \mathrm{ns}$. While some of these values may appear significant, inspection of the actual plots reveals that the actual trends are not quite so convincing, with points varying above and below the fitted line randomly.

fast rotation analysis informed the choice of $149.2 \mathrm{~ns}$. This uncertainty from the fast rotation analysis would reasonably be less than $0.1 \mathrm{~ns}$. Multiplying this uncertainty by the dataset sensitivities gives uncertainties of $2.5,0.6,2.3$, and $4.2 \mathrm{ppb}$ for the $60 \mathrm{~h}$, HighKick, 9d, and Endgame datasets respectively.

\subsubsection{Systematic error from differential decay}

Muons are injected into the storage ring with a range of momenta. Muons with larger momenta will in general live longer than muons with smaller momenta, due to their time-dilated lifetimes. Muons with smaller momenta at smaller radii therefore decay 
more often, leading to an increasing beam radial position and increasing average momentum over the course of a fill. Decay positrons from muons at greater radii in general take longer to reach the calorimeters than those from muons at small radii. In the precession frequency measurement, the hit times of the detected positrons correspond to the decay times of muons plus their drift times. Because the average drift time changes over the course of a fill due to this average momentum or radial position distribution increase, there is thus a changing phase over the course of a fill. This effect is called "differential decay." Because the differential decay effect can only increase the average stored momentum of the beam, a correction can be applied to the final measured $\omega_{a}$ value. A calculation for the previous E821 experiment resulted in a shift on $\omega_{a}$ of $\left\langle\Delta \omega_{a} / \omega_{a}\right\rangle \approx-36.8 \mathrm{ppb}[126$, which should be comparable to any calculation for E989. The systematic error applied to the precession frequency measurement is then a combination of the errors in the calculation of this shift. For the purposes of this analysis, an estimate of $25 \%$ of $40 \mathrm{ppb}$ at $10 \mathrm{ppb}$ is taken as the systematic error for all dataset:25.

\subsubsection{Systematic errors in the E-field and pitch corrections}

The electric field and pitch corrections modify the final value extracted for $\omega_{a}$ as described in Section 2.7. Any errors in the estimation of these corrections is by extension an error in the precession frequency measurement. The evaluation of these errors is performed by separate working groups. Given here are preliminary estimates of the errors.

The pitch correction is dependent on the vertical width of the muon beam and is evaluated by the tracking analysis. A preliminary analysis of the $60 \mathrm{~h}$ dataset

\footnotetext{
${ }^{25}$ It should be remembered that there is a phase-momentum correlation for muons at injection as discussed in Section 5.5.4, which affects the changing phase and corresponding error even further. Underway beam-line simulations are needed to fully measure the phase-momentum correlation and fully estimate this systematic shift.
} 


\section{Electric Field Corrections}

\begin{tabular}{lccc}
\hline \hline Dataset & Correction & Statistical error & Systematic error \\
\hline $60 \mathrm{~h}$ & -519 & 1.0 & 27.0 \\
HighKick & $\mathrm{N} / \mathrm{A}$ & $\mathrm{N} / \mathrm{A}$ & $\mathrm{N} / \mathrm{A}$ \\
$9 \mathrm{~d}$ & -463 & 1.0 & 36.0 \\
Endgame & -467 & 1.0 & 20.0 \\
\hline
\end{tabular}

Table 5.26: Electric field corrections estimated by the Cornell fast rotation analysis group $129,130,131$. Units are in ppb.

calculated an estimated correction and systematic error of 127,128

$$
C_{P} \sim-160 \pm 15 \mathrm{ppb} .
$$

This error is split into track reconstruction errors of which there are many, $\mathcal{O}(10 \mathrm{ppb})$, a tracker-calorimeter acceptance correction with a small expected error, and uncertainty in simulation models used to extract the pitch correction from the vertical width measurements. The variation in the correction between datasets is expected to be small and the error in the correction is expected to be relatively consistent. The pitch correction error given in Equation 5.44 is taken as the systematic error for all datasets.

The E-field correction is evaluated with the fast rotation analysis and is dependent on the momentum distribution of the stored muon beam. Analyses of the $60 \mathrm{~h}, 9 \mathrm{~d}$, and Endgame datasets by an independent analysis group yielded the results shown in Table 5.26. While not the final numbers for Run 1, the estimates and errors given will change only slightly from further DQC cuts. For the HighKick dataset which has not been evaluated at the time of writing, a systematic error on the E-field correction is taken as the largest error from the other datasets, that being $36 \mathrm{ppb}$ for the $9 \mathrm{~d}$ dataset.

The above corrections make certain assumptions with regards to the conditions 
which influence the stored beam dynamics. Further errors can be attributable to non-linearities in the electrostatic quadrupole field, misalignment of the quadrupole plates, the changing voltage on the quadrupole plates due to the bad resistors, and any residual radial magnetic field. Preliminary analyses using simulation have estimated additional systematic errors on the pitch and E-field corrections of $\sim 5 \mathrm{ppb}$ and $\sim 20$ ppb respectively $[132,133$. The error for the pitch correction has already been included in the number given in Equation 5.44, and the error for the E-field correction is added in quadrature with the errors given in Table 5.26. This results in E-field errors of 33.6, 41.2, 41.2, and $28.3 \mathrm{ppb}$ for the 60h, HighKick, 9d, and Endgame datasets respectively. Simulation efforts are continuing in order to assess these errors more accurately. While the combined E-field and pitch errors listed here are greater than the target quadrature sum of $30 \mathrm{ppb}$, they are nonetheless small compared to the statistical error on $R$ and are deemed acceptable for Run 1.

\subsubsection{Systematic error due to vertical beam motion}

As described in Section 4.4, due to the damaged resistors present in Run 1 the vertical distribution of the stored muon beam changed over the course of a fill. Because the $g-2$ phase is different for each vertical position due to drift length, this leads to a changing average phase and thus a systematic error ${ }^{26}$. Preliminary studies fitting the average $g-2$ phase with the tracker station 18 data have calculated systematic shifts in $\omega_{a}$ for the $60 \mathrm{~h}$ and Endgame datasets of $420 \mathrm{ppb}$ and $540 \mathrm{ppb}$ respectively 134 . Since the tracker data samples the ring in one azimuthal section, these systematic shifts need to be averaged around the ring, accounting for the oscillating beam width. This averaging factor has been calculated from simulation to be near 0.43 [135]. Multiplying these numbers together results in average systematic shifts of approximately 181 and $232 \mathrm{ppb}$ for the $60 \mathrm{~h}$ and Endgame datasets respectively.

\footnotetext{
${ }^{26}$ The acceptance also changes across the storage region which ties into the error.
} 
Since this effect is a one-directional shift in $\omega_{a}$, similar to the differential decay effect, the shifts can be applied as corrections to the final extracted precession frequencies. As systematic studies mature and the shifts for the various datasets are better quantified, this is the intended action. For now the systematic error applied from this procedure is conservatively taken as $25 \%$ of the systematic shifts themselves for the respective datasets, with the smaller number from the $60 \mathrm{~h}$ dataset being applied to the HighKick and 9d datasets as well. The $60 \mathrm{~h}$ result was chosen instead of the Endgame result because the Endgame dataset saw noticeably larger drift due to further degradation of the quadrupole resistors over the course of the run. This leads to systematic errors of $45 \mathrm{ppb}$ for the $60 \mathrm{~h}$, HighKick, and $9 \mathrm{~d}$ datasets and $58 \mathrm{ppb}$ for the Endgame dataset.

\subsubsection{Systematic error summary}

Table 5.27 gives all evaluated systematic errors in this analysis as described in the preceding sections. The final total quadrature sums of the systematic errors including the various preliminary and conservative estimates for some errors lie within the range $70-100 \mathrm{ppb}$. These are in comparison to the final target goal of $70 \mathrm{ppb}$ for the precession frequency measurement. Once the gain issues are resolved and the other working groups improve their systematic uncertainty evaluations, the systematic errors for Run 1 and future runs of E989 will very likely reach the target goal. As the systematic errors stand currently in this analysis, they are all small compared to the Run 1 statistical errors of each respective dataset, making the presented analysis statistics-limited. 


\begin{tabular}{lcccc}
\hline \multicolumn{5}{c}{ Run 1 Precession Frequency Systematic Errors } \\
\hline \hline Error & $60 \mathrm{~h}$ & HighKick & $9 \mathrm{~d}$ & Endgame \\
\hline Pileup amplitude & 22.2 & 19.0 & 9.0 & 9.4 \\
Pileup phase - time-shift & 17.6 & 19.0 & 17.1 & 14.3 \\
Pileup phase - energy-scale & 19.4 & 3.7 & 5.5 & 5.3 \\
In-fill gain amplitude & 1.4 & 28.6 & 0.4 & 43.6 \\
In-fill gain lifetime & 5.0 & 11.2 & 11.6 & 16.5 \\
STDP On/Off & $\sim 11.0$ & $\sim 11.0$ & $\sim 11.0$ & $\sim 11.0$ \\
CBO frequency model & 7.5 & 0.4 & 2.0 & 8.0 \\
CBO decoherence envelope & 17.6 & 18.0 & 9.3 & 4.3 \\
Lost muon cuts & $<0.5$ & $<0.5$ & $<0.5$ & $<0.5$ \\
Fixed $\kappa_{\text {loss }}$ & 1.2 & 4.9 & 3.1 & 0.1 \\
Ratio construction $T_{a}$ & 2.2 & 2.2 & $<2.2$ & $<2.2$ \\
Ratio construction $\tau_{\mu}$ & $<0.1$ & $<0.1$ & $<0.1$ & $<0.1$ \\
Bin width & 2.5 & 0.6 & 2.3 & 4.2 \\
\hline Quadrature Sum & 41.3 & 46.4 & 27.8 & 52.2 \\
\hline \hline Lost muon phase bias & 53.6 & 17.9 & 22.3 & 58.0 \\
Differential decay & $\sim 10$ & $\sim 10$ & $\sim 10$ & $\sim 10$ \\
Pitch correction & 15 & $\sim 15$ & $\sim 15$ & $\sim 15$ \\
E-field correction & 33.6 & $\sim 41.2$ & 41.2 & 28.3 \\
Vertical beam motion & $\sim 45$ & $\sim 45$ & $\sim 45$ & $\sim 58$ \\
\hline Quadrature Sum & 79.7 & 66.1 & 67.4 & 88.6 \\
\hline \hline Total Quadrature Sum & 89.8 & 80.8 & 72.9 & 102.8 \\
\hline
\end{tabular}

Table 5.27: Final systematic errors evaluated in the Run 1 precession frequency analysis to the 60h, HighKick, 9d, and Endgame datasets. All units are in ppb. The table is split into two sections. The upper section consists of systematic errors directly evaluated by the author while the lower section consists of preliminary systematic estimates by other working groups. The final errors for the Run 1 datasets will change from these as the final DQC cuts are made and analyses improved, however the scale of these errors is expected to remain consistent. 


\subsection{Final precession frequency results}

Four of five datasets from Run 1 of E989 have been analyzed for this dissertation, those being the 60h, HighKick, 9d, and Endgame 27 . In each case the datasets are the near-final datasets for Run 1. The precession frequency analysis was done using the Ratio Method, an analysis technique for fitting the decay positron time spectra which divides out the exponential decay along with slow and smooth terms in the data. The final results for the blinded frequency $R$ values for the different datasets along with their total statistical and systematic errors are given in Table 5.28. Figure $5 \cdot 49$ compares the $R$ values between datasets. The total error for the four datasets is $469.4 \mathrm{ppb}$, conservatively assuming completely correlated systematic errors. The analysis is statistics limited, even with the conservative preliminary estimates for certain systematic errors as given in Table 5.27. Before combining the results, the $R$ values given here will have their individual E-field and pitch corrections applied, and their blindings normalized. Since the magnetic field changes between datasets, the $R$ values will be converted back into the precession frequency $\omega_{a}$ using Equation 5.14 , before combination with the magnetic field measurements to give per-dataset values of $a_{\mu}$. The dataset $a_{\mu}$ values will then be combined in a weighted average.

\footnotetext{
${ }^{27}$ The fifth dataset as a reminder is the LowKick dataset, which contains less stats than the rest of the datasets, but which will most likely be included in the final Run 1 result.
} 


\begin{tabular}{lcccc}
\hline \multicolumn{4}{c}{ Run 1 Precession Frequency Results } \\
\hline \hline Dataset & $R$ & $\sigma_{\text {stat. }}$ & $\sigma_{\text {sys. }}$ & $\sigma_{\text {tot. }}$ \\
\hline 60h & -20.5562 & 1.3581 & 0.0898 & 1.3611 \\
HighKick & -17.4755 & 1.4112 & 0.0808 & 1.4135 \\
9d & -17.7182 & 0.9033 & 0.0729 & 0.9062 \\
Endgame & -17.3406 & 0.6393 & 0.1028 & 0.6475 \\
\hline \multicolumn{2}{c}{ Total (systematics $100 \%$ correlated) } & 0.4605 & & 0.4694 \\
\hline
\end{tabular}

Table 5.28: Run 1 final results for the precession frequency analysis datasets. The $R$ values given here are mean values of fits to 50 different random seeds. The $60 \mathrm{~h}$ dataset has a different software blinding than the rest, shown by the different mean $R$ value. Statistical and systematic errors are included alongside the total error for each dataset. In each dataset case the error is statistics dominated. The total combined error for all datasets is shown for the case where the systematic errors are assumed to be $100 \%$ correlated. Units are in ppm.

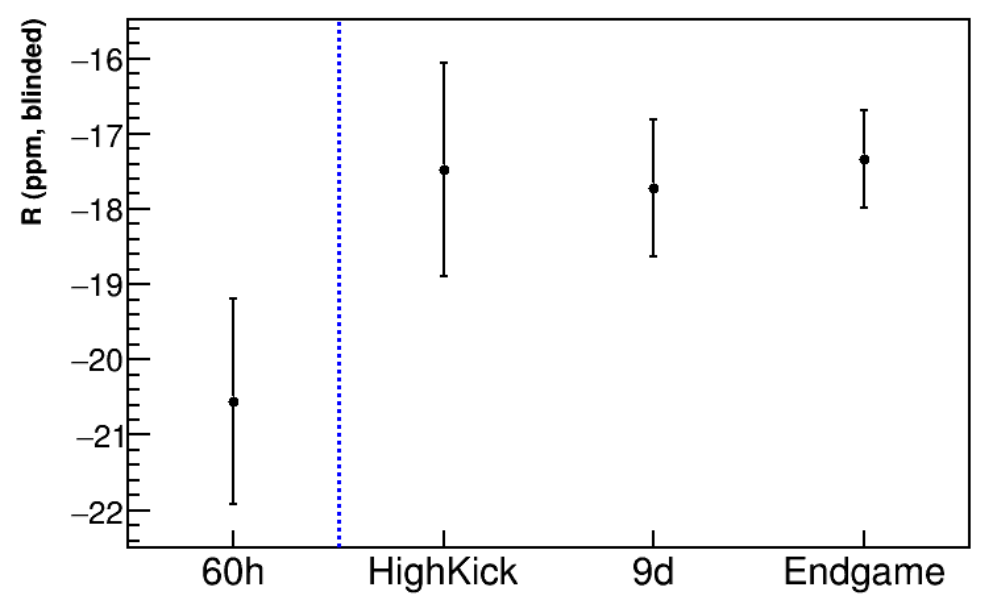

Figure 5.49: Comparison between dataset mean $R$ values from fits to 50 different random seeds. The $60 \mathrm{~h}$ dataset has a different software blinding than the rest of the datasets, so it's mean value cannot be directly compared to the rest. 


\section{Chapter 6}

\section{Conclusion}

Experiment E821 measured the anomalous magnetic moment of the muon, $a_{\mu}$, to a relative uncertainty of 540 parts per billion. The measured value corresponds to a discrepancy of three to four standard deviations from the Standard Model theoretical value, depending on the chosen theory. In order to verify this discrepancy, a new experiment based upon the same principles has been undertaken to measure the same quantity with greater precision. Fermilab Muon $g-2$, E989, measures $a_{\mu}$ by measuring the spin precession frequency of muons within a magnetic storage ring, and the strength of the magnetic field that the muons experience. The former is done by counting the number of decay positrons observed in electromagnetic calorimeters above an energy threshold, while the latter is done using NMR probes in and around the muon storage region.

Straw trackers assist both measurements by measuring muon beam dynamics which directly impact both the precession frequency measurement and the distribution of muons within the measured magnetic field. In Chapter 4 this dissertation presented the track fitting algorithm used in the reconstruction of decay positron tracks, necessary for reconstructing muon beam decay vertices. The track fitting method, Geane, propagates positrons in the full E989 Geant4 simulation, with the advantage of direct access to the geometry, material, and non-uniform magnetic field present within the tracker region. Transport matrices, error matrices, and predicted parameter vectors are generated which are used in a global $\chi^{2}$ minimization algo- 
rithm, to produce optimal state vectors at the entrance to the tracker. These state vectors are extrapolated back into the storage region to approximate muon decay vertices, from which the muon distribution and beam motion can be derived. The beam motion was observed to be coherent, such that the muons as a whole oscillate within the storage region. This coherent motion has been well characterized both radially and vertically. These radial and vertical oscillations introduce modulations on top of the $\omega_{a}$ signal seen in the decay positron time spectra. Due to damaged quadrupole resistors in Run 1, the frequency of these oscillations was found to be changing over time. Both the modulations and the changing frequency effect were included in the precession frequency analysis. Lastly, the equilibrium vertical distribution of the muons ties directly into the pitch correction which shifts the measured $\omega_{a}$ frequency by $\mathcal{O}(100 \mathrm{ppb})$. Preliminary analysis of the $60 \mathrm{~h}$ dataset has determined a value of $-160 \pm 15$ ppb. Numerous plots regarding the beam distribution and dynamics can be found in Section 4.4 .

Muon decay is a self-analyzing process, meaning that decay positrons retain muon spin information. The correlation between the emission direction of high energy decay positrons and the muon spin at the time of the decay provides the signal with which to measure the muon spin precession frequency. Decay positrons above an energy threshold are counted and put into a time histogram, from which the $\omega_{a}$ oscillation can be extracted. The time spectrum is corrected for gain variations and the pileup background which distort the $g-2$ signal. The precession frequency in this analysis is extracted using a technique called the Ratio Method, detailed in Chapter 5. The Ratio Method works by splitting the positron decay time spectrum into four subsets, time-shifting two of them, and taking the ratio of the difference and sum of the shifted and un-shifted datasets respectively. This procedure removes the muon decay exponential from the data while also reducing any slowly and smoothly varying effects 
present in the data. Precession frequency extraction analyses for four near-final Run 1 datasets were presented with full systematic error evaluations. Fit functions including terms which account for various beam dynamics effects have been applied to the data with success. The integrity of the fits have been checked by splitting up the data in numerous ways, and verifying that fit results remain consistent regardless of detector number, fit start time, energy threshold, bunch number, etc. Systematic errors were evaluated with respect to the assumptions made regarding beam dynamics terms, the subtraction of the pileup background, treatment of the gain variations, and more. The systematic errors are well understood, with independent groups currently working on improving the largest outstanding systematic errors. Preliminary estimates of the E-field corrections are $-519 \pm 27,-463 \pm 36$, and $-467 \pm 20 \mathrm{ppb}$, for the $60 \mathrm{~h}, 9 \mathrm{~d}$, and Endgame datasets respectively. Summary tables for the systematic errors and final extracted precession frequency values can be found in Tables 5.27 and 5.28 . The total Run 1 precession frequency error determined in this analysis is $469.4 \mathrm{ppb}$, where the error is statistics dominated.

The expected error in the magnetic field measurement for Run 1 is $\mathcal{O}(140 \mathrm{ppb})$. Combined with the total precession frequency error determined in this analysis, the error on $a_{\mu}$ is expected to be $\mathcal{O}(500 \mathrm{ppb})$, comparable to the uncertainty in the E821 measurement. For the final Run 1 production datasets with the last data quality cuts and gain improvements, the final errors are expected to improve only slightly. An independent measurement of $a_{\mu}$ that is statistically consistent with the E821 result would go a long way towards increasing the confidence in the discrepancy between theory and experiment. The Run 1 publication is expected to be complete sometime in 2020. Data has already been gathered for Run 2, Run 3 has just begun in the late fall of 2019, and Run 4 is planned to run from late 2020 to the middle of 2021. With the rate improvements seen in Run 2 and the expected increases for Run 3 
and Run 4, the target uncertainty goal of $140 \mathrm{ppb}$ is a likely reality. Assuming the same central value for $a_{\mu}$ is measured as was done in the previous experiment, the statistical significance of the discrepancy between the theory and experiment would be pushed over five standard deviations, providing strong evidence for the existence of new physics, and constraining any explanatory models. 


\section{Appendix A}

\section{Tracking Derivations}

\section{A.1 Straw measurement angular correction}

The tracker straws do not measure $\mathrm{U}$ and V coordinates directly, but instead measure the DCA radii derived from measured hit times. In order to utilize the minimization procedure on measured track parameters described in Section 4.2.2, these radii must first be converted to U and V parameters, and similarly for the U and V errors. While to first order the measured DCAs can be used identically as the $\mathrm{U}$ and $\mathrm{V}$ positions, it was found that there were slight biases in the truth pulls.

In order to improve the results, angular corrections were made to the DCAs to give more accurate estimates of the "measured" positions. It was found that for the error correction, assuming a straight particle path was sufficient for ideal results. For the position correction, it was found that assuming a circular particle path (constant field) correction for the curved tracks was sufficient. These corrections are dependent on the angle of the track, so it's important to note that during each successive iteration of the track fitting, the "measured" parameters are adjusted by the latest "predicted" momenta, which change the angle of the track. The correction depends on whether the track went to the left or right side of the wire. Note that the momentum perpendicular to the straw measurement axis can be ignored since it does not affect the $\mathrm{U}$ or $\mathrm{V}$ value. A summary of the calculation of the right side correction follows, with the left side correction being calculated in a similar manner. Figure $\mathrm{A} \cdot 1$ provides a diagram showing the parameters in the calculation. 


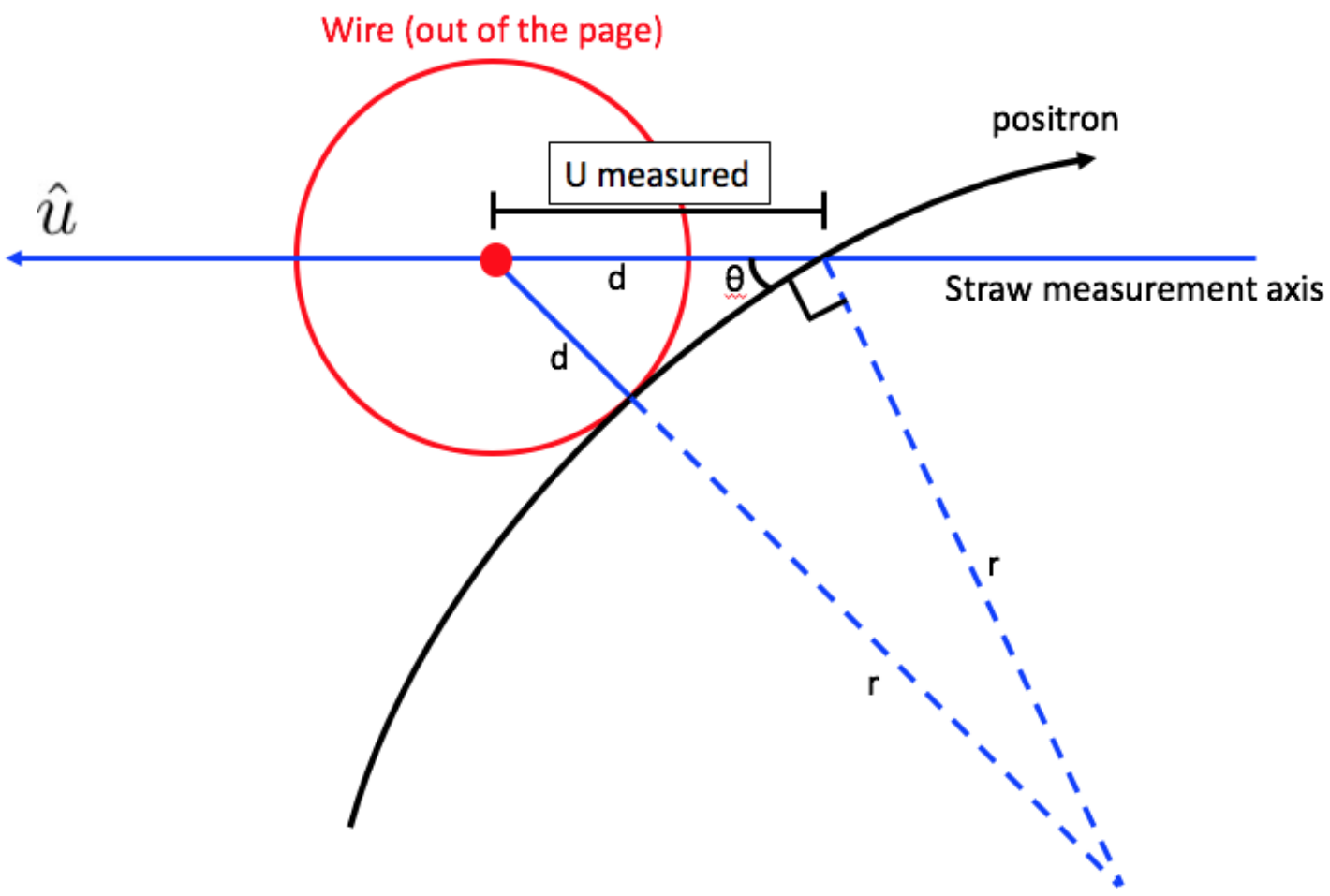

Figure A.1: A positron passing through a straw will produce a hit of radius $d$. The desired value is the $\mathrm{U}$ or $\mathrm{V}$ position along the straw measurement axis. The positron trajectory can be approximated as a circle in a constant magnetic field over the length of the path across the straw. The curvature for the high energy positrons is small such that $r \gg d$ and the angle between the trajectory and the center of the circle can be approximated as $90^{\circ}$. Sizes and angles are exaggerated. A similar diagram can be drawn for positrons passing to the left of the wire. 
To solve for the measured $\mathrm{U}$ (or $\mathrm{V}$ ) value, first use the following trigonometric identity:

$$
(r+d)^{2}=r^{2}+U^{2}-2 r U \cos (90+\theta)
$$

where $U$ is the parameter of interest. The angle $\theta$ can be determined from

$$
\hat{z_{\|}} \cdot \hat{p_{\|}}=\cos \theta, \theta=\cos ^{-1} \frac{p_{\|}}{p}
$$

where $p_{\|}$is the positron momentum anti-parallel to the $\mathrm{U}$ measurement axis at the wire plane, and $p$ is the total momentum. Using other trigonometric identities and solving for $U$ gives

$$
U=-r \sqrt{1-\left(\frac{p_{\|}}{p}\right)^{2}}+\sqrt{d^{2}+2 d r+r^{2}\left(1-\left(\frac{p_{\|}}{p}\right)^{2}\right)}
$$

for the right side correction. Similarly,

$$
U=+r \sqrt{1-\left(\frac{p_{\|}}{p}\right)^{2}}-\sqrt{d^{2}+2 d r+r^{2}\left(1-\left(\frac{p_{\|}}{p}\right)^{2}\right)}
$$

for the left side correction. The radius $r$ can be calculated from the momentum and magnetic field at the predicted hit position, and the momentum components can be determined within the Geant4 simulation. The straight line correction to the errors is done in a simpler manner using the Pythagorean theorem, such that

$$
\sigma_{U V}^{\prime}=\frac{\sigma_{U V}}{\sqrt{1-\left(\frac{p_{\|}}{p}\right)^{2}}}
$$

where $\sigma_{U V}^{\prime}$ is the improved error from the original error $\sigma_{U V}$ on the DCA. 


\section{A.2 Track fitting correlation matrices}

Define $\epsilon_{M N}$ as the deviation in average predicted track parameters from the true ones on plane $M$, from track parameters propagated from plane $N$, as

$$
\epsilon_{M N}=\widetilde{p}_{M}\left(p_{N}\right)-p_{M}
$$

where $N<M$ can be any plane numbers. From the definition of transport matrices in Equation 4.6 ,

$$
\epsilon_{M 0}=T_{M N} \epsilon_{N 0}+\epsilon_{M N}
$$

where $\epsilon_{M N}$ is any additional deviation between planes $N$ and $M$ due to material effects. By definition, the correlation between error matrices on planes $M$ and $N$ is given by

$$
\sigma_{M N}=\left\langle\epsilon_{M 0} \epsilon_{N 0}\right\rangle=\left\langle\epsilon_{M 0} \cdot \epsilon_{N 0}\right\rangle-\left\langle\epsilon_{M 0}\right\rangle \cdot\left\langle\epsilon_{N 0}\right\rangle
$$

The last term in Equation A.8 is zero since $\left\langle\epsilon_{M 0}\right\rangle$ and $\left\langle\epsilon_{N 0}\right\rangle$ are both individually zero. Therefore

$$
\sigma_{M N}=\left\langle\left(T_{M N} \epsilon_{N 0}+\epsilon_{M N}\right) \cdot \epsilon_{N 0}\right\rangle
$$

and since $\epsilon_{M N}$ and $\epsilon_{N 0}$ are independent, such that their product is zero,

$$
\sigma_{M N}=T_{M N} \sigma_{N}
$$

since $\sigma_{N}=\left\langle\epsilon_{N 0} \cdot \epsilon_{N 0}\right\rangle$. 


\section{Appendix B}

\section{Ratio Method Derivation}

\section{B.1 Ratio form and function}

Consider the 5 parameter function:

$$
N_{5}(t)=N_{0} e^{-t / \tau}\left(1+A \cos \left(\omega_{a} t+\phi\right)\right)
$$

which describes some ideal dataset in histogram format. Here $\phi$ will be set to zero for simplicity. Now define the variables $u_{+}(t), u_{-}(t), v_{1}(t)$, and $v_{2}(t)$ as

$$
\begin{aligned}
& u_{+}(t)=\frac{1}{4} N_{5}(t+T / 2), \\
& u_{-}(t)=\frac{1}{4} N_{5}(t-T / 2), \\
& v_{1}(t)=\frac{1}{4} N_{5}(t), \\
& v_{2}(t)=\frac{1}{4} N_{5}(t),
\end{aligned}
$$

where the $1 / 4$ out front reflects randomly splitting the whole dataset into 4 equally weighted sub-datasets, and $T$ is the $g-2$ period known a priori to high precision, $\mathcal{O}\left(10^{-6}\right)$. This corresponds to a weighting of 1:1:1:1 between the datasets. To be explicit here regarding the signs, the counts that are filled into the histogram described by $u_{+}$have their times shifted as $t \rightarrow t-T / 2$, which is what the function $N_{5}(t+T / 2)$ 
describes, and vice versa for $u_{-}$. To form the ratio define the variables:

$$
\begin{aligned}
U(t) & =u_{+}(t)+u_{-}(t), \\
V(t) & =v_{1}(t)+v_{2}(t), \\
R(t) & =\frac{V(t)-U(t)}{V(t)+U(t)} .
\end{aligned}
$$

Plugging in and dividing the common terms $\left(N_{0} e^{-t / \tau} / 4\right)$,

$$
\begin{aligned}
& 2\left(1+A \cos \left(\omega_{a} t\right)\right)-e^{-T / 2 \tau}\left(1+A \cos \left(\omega_{a} t+\omega_{a} T / 2\right)\right) \\
& R(t)=\frac{-e^{T / 2 \tau}\left(1+A \cos \left(\omega_{a} t-\omega_{a} T / 2\right)\right)}{2\left(1+A \cos \left(\omega_{a} t\right)\right)+e^{-T / 2 \tau}\left(1+A \cos \left(\omega_{a} t+\omega_{a} T / 2\right)\right)} . \\
& +e^{T / 2 \tau}\left(1+A \cos \left(\omega_{a} t-\omega_{a} T / 2\right)\right)
\end{aligned}
$$

Now set $\omega_{a} T / 2=\delta$, and note that $T$ is really

$$
\begin{array}{r}
T=T_{\text {guess }}=\frac{2 \pi}{\omega_{a}}+\Delta T \\
\Delta T=T_{\text {guess }}-T_{\text {true }}
\end{array}
$$

Being explicit,

$$
\delta=\frac{\omega_{a}}{2} T_{\text {guess }}=\frac{\omega_{a}}{2}\left(\frac{2 \pi}{\omega_{a}}+\Delta T\right)=\pi+\pi \frac{\Delta T}{T_{\text {true }}}=\pi+\pi(\delta T)
$$

and $\delta$ can be redefined as

$$
\delta=\pi(\delta T)
$$

by flipping the sign of any cosine terms that contain $\delta$.

Then, using the trig identity

$$
\cos (a \pm b)=\cos (a) \cos (b) \mp \sin (a) \sin (b)
$$


so that

$$
\begin{aligned}
\cos \left(\omega_{a} t \pm \delta\right) & =\cos \left(\omega_{a} t\right) \cos \delta \mp \sin \left(\omega_{a} t\right) \sin \delta \\
& \approx \cos \left(\omega_{a} t\right)\left(1-\delta^{2}\right) \mp \sin \left(\omega_{a} t\right) \delta \\
& \approx \cos \left(\omega_{a} t\right)
\end{aligned}
$$

since $\delta \sim O\left(10^{-5}\right)$, the ratio becomes

$$
R(t) \approx \frac{2\left(1+A \cos \left(\omega_{a} t\right)\right)-\left(1-A \cos \left(\omega_{a} t\right)\right)\left(e^{-T / 2 \tau}+e^{T / 2 \tau}\right)}{2\left(1+A \cos \left(\omega_{a} t\right)\right)+\left(1-A \cos \left(\omega_{a} t\right)\right)\left(e^{-T / 2 \tau}+e^{T / 2 \tau}\right)}
$$

Expanding

$$
e^{ \pm T / 2 \tau}=1 \pm \frac{T}{2 \tau}+\frac{1}{2}\left(\frac{T}{2 \tau}\right)^{2} \pm \ldots
$$

replacing and simplifying,

$$
R(t) \approx \frac{A \cos \left(\omega_{a} t\right)-C\left(1-A \cos \left(\omega_{a} t\right)\right)}{1+C\left(1-A \cos \left(\omega_{a} t\right)\right)}
$$

where

$$
C=\frac{1}{16}\left(\frac{T}{\tau}\right)^{2} \approx 2.87 \times 10^{-4}
$$

Using the expansion

$$
f(x)=\frac{1}{1+x}=1-x+x^{2}-\ldots, \quad|x|<1,
$$

and since $C$ is small, the denominator can be manipulated such that

$$
\begin{aligned}
R(t) & \approx A \cos \left(\omega_{a} t\right)-C\left(1-A \cos \left(\omega_{a} t\right)\right)\left(1-C\left(1-A \cos \left(\omega_{a} t\right)\right)\right) \\
& \approx A \cos \left(\omega_{a} t\right)-C+C A^{2} \cos ^{2}\left(\omega_{a} t\right)
\end{aligned}
$$

after dropping terms of $\mathcal{O}\left(C^{2}\right)$ and higher. In practice the last term is omitted since 
it has a minimal effect on the fitted value of $\omega_{a}[109]$, and one arrives at

$$
R(t) \approx A \cos \left(\omega_{a} t\right)-C
$$

the conventional 3 parameter ratio function.

In order to avoid approximations one can instead weight the counts in the histograms as

$$
u_{+}(t): u_{-}(t): v_{1}(t): v_{2}(t)=e^{T / 2 \tau}: e^{-T / 2 \tau}: 1: 1,
$$

so that

$$
\begin{aligned}
& u_{+}(t)=\frac{e^{T / 2 \tau}}{2+e^{T / 2 \tau}+e^{-T / 2 \tau}} N_{5}(t+T / 2), \\
& u_{-}(t)=\frac{e^{-T / 2 \tau}}{2+e^{T / 2 \tau}+e^{-T / 2 \tau}} N_{5}(t-T / 2), \\
& v_{1}(t)=\frac{1}{2+e^{T / 2 \tau}+e^{-T / 2 \tau}} N_{5}(t), \\
& v_{2}(t)=\frac{1}{2+e^{T / 2 \tau}+e^{-T / 2 \tau}} N_{5}(t) .
\end{aligned}
$$

(These factors out front are close to $1 / 4$ since $e^{ \pm T / 2 \tau} \approx e^{ \pm 4.35 / 2 * 64.4} \approx 1.034, .967$.) Then instead $R(t)$ becomes

$$
R(t)=\frac{2\left(1+A \cos \left(\omega_{a} t\right)\right)-\left(1-A \cos \left(\omega_{a} t+\delta\right)\right)-\left(1-A \cos \left(\omega_{a} t-\delta\right)\right)}{2\left(1+A \cos \left(\omega_{a} t\right)\right)+\left(1-A \cos \left(\omega_{a} t+\delta\right)\right)+\left(1-A \cos \left(\omega_{a} t-\delta\right)\right)}
$$

where the $e^{ \pm T / 2 \tau}$ terms out front now cancel. Using Equation B.9 again and this time avoiding approximations in $\delta$,

$$
R(t)=\frac{2 A \cos \left(\omega_{a} t\right)(1+\cos \delta)}{4+2 A \cos \left(\omega_{a} t\right)(1-\cos \delta)},
$$

after simplifying. In the limit that

$$
\delta=\pi(\delta T) \rightarrow 0
$$


since $\delta T$ is small,

$$
R(t) \approx A \cos \left(\omega_{a} t\right)
$$

with the only approximation being made at $\mathcal{O}\left(\delta^{2}\right) \sim \mathcal{O}\left(10^{-10}\right)$.

Finally, while the 3 parameter ratio function suffices for fits to data containing slow modulations, it does not suffice for faster oscillation features. In that case it is more useful to fit with the non-approximated or simplified version of the ratio,

$$
\begin{aligned}
R(t) & =\frac{v_{1}(t)+v_{2}(t)-u_{+}(t)-u_{-}(t)}{v_{1}(t)+v_{2}(t)+u_{+}(t)+u_{-}(t)} \\
& =\frac{2 f(t)-f_{+}(t)-f_{-}(t)}{2 f(t)+f_{+}(t)+f_{-}(t)}
\end{aligned}
$$

where

$$
\begin{aligned}
f(t) & =C(t)\left(1+A \cos \left(\omega_{a} t+\phi\right)\right) \\
f_{ \pm}(t) & =f\left(t \pm T_{a} / 2\right)
\end{aligned}
$$

and $C(t)$ can encode any other effects in the data that need to be fitted for, such as the $\mathrm{CBO}$,

$$
C(t)=1+A_{c b o} \cdot e^{-t / \tau_{c b o}} \cdot \cos \left(\omega_{c b o} t+\phi_{c b o}\right)
$$

Additionally, any other fit parameters such as $A$ or $\phi$ can be made a function of $t$. Using the non-approximated form for the final fit function gives greater confidence in the fit results for the high precision $\omega_{a}$ extraction necessary for the experimental measurement. 


\section{B.2 Ratio errors}

In order to determine the errors on the points in the formed ratio, Equation B.3. standard error propagation is used:

$$
\sigma_{R}(t)^{2}=\left(\frac{\partial R(t)}{\partial V(t)}\right)^{2} \delta V(t)^{2}+\left(\frac{\partial R(t)}{\partial U(t)}\right)^{2} \delta U(t)^{2}
$$

This works because $V(t)$ and $U(t)$ are statistically independent datasets. Using standard error propagation again,

$$
\begin{aligned}
& \delta V(t)^{2}=\delta v_{1}(t)^{2}+\delta v_{2}(t)^{2}=v_{1}(t)+v_{2}(t)=V(t) \\
& \delta U(t)^{2}=\delta u_{+}(t)^{2}+\delta u_{-}(t)^{2}=u_{+}(t)+u_{-}(t)=U(t)
\end{aligned}
$$

Calculating out and simplifying the partial derivatives, (dropping the time-dependencies),

$$
\begin{aligned}
& \frac{\partial R}{\partial V}=\frac{2 U}{(V+U)^{2}}, \\
& \frac{\partial R}{\partial U}=\frac{-2 V}{(V+U)^{2}} .
\end{aligned}
$$

Combining and simplifying, the error formula is given as:

$$
\sigma_{R}^{2}=\frac{4 U V}{(V+U)^{3}}=\frac{1-R^{2}}{(V+U)}
$$




\section{Appendix C}

\section{Pileup Modified Errors}

In the pileup subtraction method detailed in Section 5.2.1, pileup events are statistically constructed and then subtracted from the data. Because of this, the errors on the bins need to be adjusted appropriately. Reference [136 describes the modified errors, but is not quite correct. Here is provided an improved calculation that is easier to understand. While we are mainly interested in the errors on the histogram bins after pileup subtraction, it first helps to examine the errors of the pileup histogram itself. Here we only consider doublets.

In the asymmetric shadow window pileup method, shadow doublets are constructed from two singlets. The pileup histogram is then filled as the sum of the doublets minus the singlets,

$$
P=D-S
$$

where $D$ or $S$ are only added or subtracted when they are above some energy threshold. If the threshold is set to zero, then for every doublet one entry will be added and two will be subtracted. Since these entries are exactly correlated, the error in each time bin will be

$$
\sigma_{P}=\sqrt{N_{D}}
$$

where $N_{D}$ is the number of doublets in that time bin. If the energy threshold is above zero, then we can determine whether the counts in the pileup histogram increase or 


\begin{tabular}{lll}
\hline & $E_{1}<E_{t h}$ & $E_{1}>E_{t h}$ \\
\hline$E_{2}<E_{t h}$ & $N_{1}(+1)$ & $N_{2}(0)$ \\
$E_{2}>E_{t h}$ & $N_{3}(0)$ & $N_{4}(-1)$
\end{tabular}

Table C.1: Table of doublets above threshold. Here $E_{1}$ and $E_{2}$ are the energies of the two singlets, $E_{t h}$ is the energy threshold, and $N_{i}$ are the number of doublets above threshold for the different combinations of $E_{1}$ and $E_{2}$. ( $N_{1}$ is assumed above threshold here.) The numbers in the parentheses indicate the number of counts gained or lost in the pileup histogram.

decrease based on whether the singlets and doublets are above threshold or not. Table C.1 shows the different combinations of counts put into the pileup histogram. The counts that go into $P$ will be

$$
\begin{aligned}
P & =\sum_{i} N_{i}-\text { singlets above threshold } \\
& =\left(N_{1}+N_{2}+N_{3}+N_{4}\right)-\left(N_{2}+N_{4}\right)-\left(N_{3}+N_{4}\right) \\
& =N_{1}-N_{4}
\end{aligned}
$$

and the errors are

$$
\sigma_{P}=\sqrt{N_{1}+N_{4}}
$$

Consider the individual cases: In the cases for $N_{1}$, you will gain a count from the doublet above threshold, and lose no counts since both singlets are below threshold. In the cases for $N_{2}$ and $N_{3}$, you will gain a count from the doublet, and lose a count from one of the singlets which is above threshold. In the cases for $N_{4}$, you will gain a count from the doublet and lose two counts from the singlets which are both above threshold. Since the doublet and singlets are exactly correlated, the $N_{1}$ and $N_{4}$ cases naturally result in a single weight being added into the error, while the $N_{2}$ and $N_{3}$ cases result in no additions to the error. 
Consider now the pileup subtracted time spectrum. The corrected spectrum can be written as

$$
N_{\text {corrected }}=N_{\text {measured }}-P \text {. }
$$

The content in $N_{\text {measured }}$ does not matter exactly. What matters is what is in $N_{\text {measured }}$ that is also within $P$, for that is where the correlations come from. Since $N_{\text {measured }}$ is the sum of all singlets above threshold, we can write it as

$$
N_{\text {measured }}=N_{\text {other }}+N_{2}+N_{3}+2 N_{4}
$$

since we know that those cases $N_{i}$ listed come from singlets above threshold, and $N_{\text {other }}$ is anything in the measured hits that was not included in the pileup shadow construction. We can then replace $P$ and simplify to get

$$
N_{\text {corrected }}=N_{\text {other }}-N_{1}+N_{2}+N_{3}+3 N_{4} .
$$

The error on the corrected histogram is then

$$
\sigma_{N_{\text {corrected }}}=\sqrt{N_{\text {other }}+N_{1}+N_{2}+N_{3}+9 N_{4}} .
$$

Replacing $N_{\text {other }}$ as

$$
N_{\text {other }}=N_{\text {corrected }}+N_{1}-N_{2}-N_{3}-3 N_{4} \text {, }
$$

we can remove the dependence of the corrected histogram errors on the unknown quantity and arrive at

$$
\begin{aligned}
\sigma_{N_{\text {corrected }}} & =\sqrt{N_{\text {corrected }}+2 N_{1}+6 N_{4}}, \\
& =\sqrt{N_{\text {corrected }}} \cdot \sqrt{1+\left(2 N_{1}+6 N_{4}\right) / N_{\text {corrected }}} .
\end{aligned}
$$


(This argument might seem circular at the end, but it works because of the squaring that occurs when calculating the error.) In the end we have a form for the bin errors of the pileup corrected histogram which only depend on $N_{1}$ and $N_{4}$ in addition to the number of counts in the corrected histogram. As shown it can be re-factored into a form equal to the naive errors (just the bin content) times some correction factor. Since $N_{1}$ and $N_{4}$ are much smaller than $N_{\text {corrected }}$ at all times, and because they decay away at about twice the rate as the pileup diminishes, the change to the errors is small, of the order 1 or $2 \%$ at $30 \mu \mathrm{s}$.

\section{C.1 Pileup errors for the ratio function}

Equation C.10 applies to the corrected errors for a pileup subtracted histogram, but what about the modifications to the ratio errors? If we parameterize that equation as

$$
\sigma_{N_{\text {corrected }}}=\sqrt{N_{\text {corrected }}} \cdot \sqrt{\gamma(t)}
$$

where the correction factor $\gamma(t) \approx \gamma e^{-t / \tau_{\mu}}$ is small and decays at approximately the muon lifetime, we can recast the errors on the individual ratio sub-datasets as

$$
\begin{aligned}
\delta V(t)^{2} & =\delta v_{1}(t)^{2} \cdot \gamma(t)+\delta v_{2}(t)^{2} \cdot \gamma(t)=\left(v_{1}(t)+v_{2}(t)\right) \cdot \gamma(t)=V(t) \cdot \gamma(t) \\
\delta U(t)^{2} & =\delta u_{+}(t)^{2} \cdot \gamma(t+T / 2)+\delta u_{-}(t)^{2} \cdot \gamma(t-T / 2) \\
& \approx u_{+}(t) \cdot \gamma(t) e^{-T / 2 \tau}+u_{-}(t) \cdot \gamma(t) e^{+T / 2 \tau} \\
& \approx\left(u_{+}(t)+u_{-}(t)\right) \cdot \gamma(t) \cdot\left(1+\frac{1}{2}\left(\frac{T}{2 \tau}\right)^{2}\right) \\
& \approx U(t) \cdot \gamma(t)
\end{aligned}
$$

where in the last step the $\frac{1}{2}\left(\frac{T}{2 \tau}\right)^{2}$ term has been neglected because it's small. With these approximations having been made, the modified errors on the ratio points simply 
become

$$
\sigma_{R}^{2} \rightarrow \sigma_{R}^{2} \cdot \gamma(t)
$$

with the correction being the same as that on the pileup subtracted histogram. Credit to J. Paley for this derivation [110]. 


\section{Appendix D}

\section{Fit Result Correlation Matrices}

Correlation matrices for the 60h, HighKick, 9d, and Endgame datasets are included here, for single seed fits to the calorimeter sum data. In the matrices the rows and columns for the $\kappa_{\text {loss }}$ parameter are blank as the parameter is fixed from T-Method fits to the same data. The only parameter that is significantly correlated with $R$ is the $g-2$ phase. Correlations between CBO parameters can be seen, and it was found that there were stronger correlations between higher order $\mathrm{CBO}$ parameters in the HighKick and $9 \mathrm{~d}$ datasets than in the $60 \mathrm{~h}$ and Endgame datasets. 


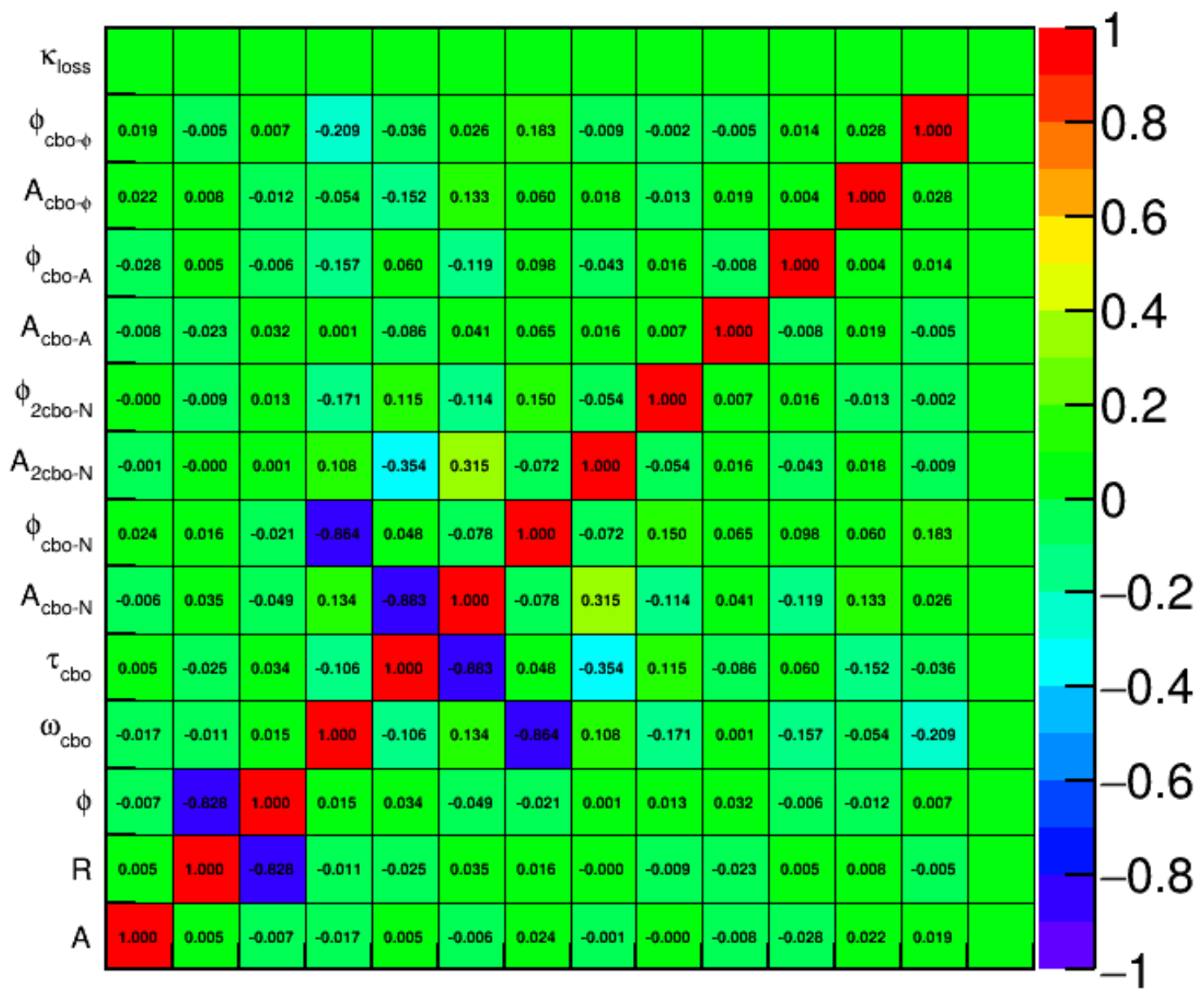

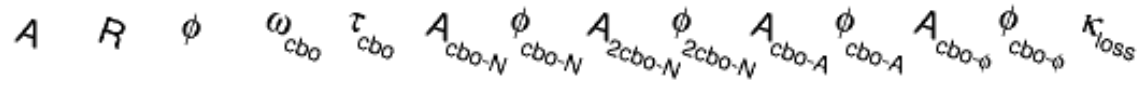

Figure D-1: Correlation matrix for a single seed ratio fit to the $60 \mathrm{~h}$ dataset. The only significant correlation with $R$ is the $g-2$ phase. $\kappa_{\text {loss }}$ is fixed, hence the corresponding empty row and column. 


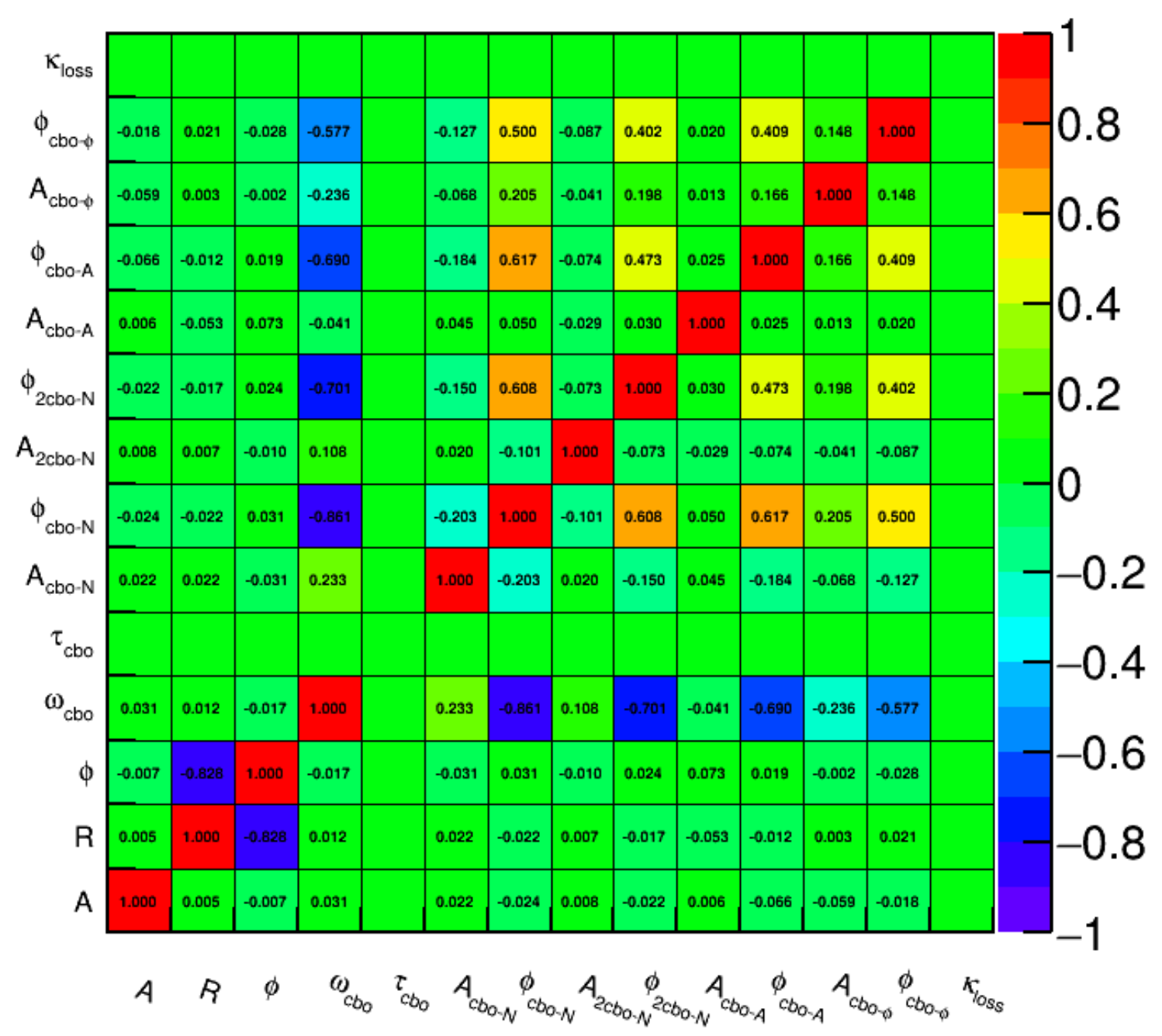

Figure D.2: Correlation matrix for the single seed ratio fit to the HighKick dataset. The only significant correlation with $R$ is the $g-2$ phase. $\tau_{c b o}$ and $\kappa_{\text {loss }}$ are fixed, hence the corresponding empty rows and columns. 


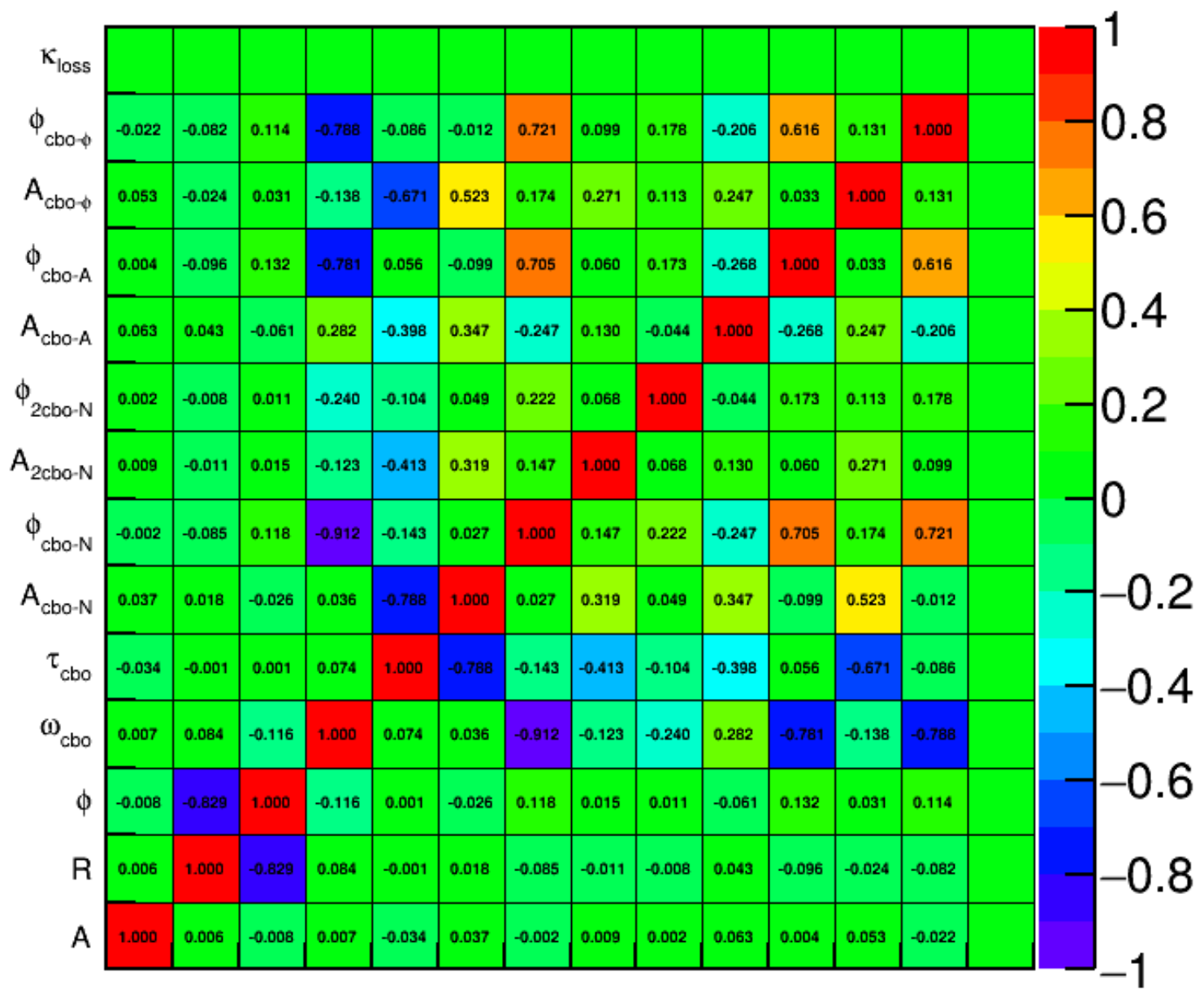

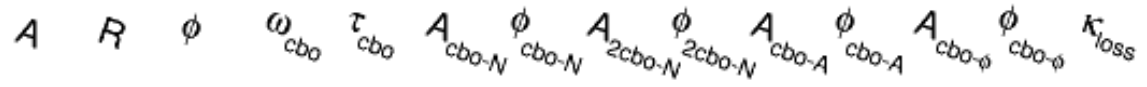

Figure D.3: Correlation matrix for the single seed ratio fit to the $9 \mathrm{~d}$ dataset. The only significant correlation with $R$ is the $g-2$ phase. $\kappa_{\text {loss }}$ is fixed, hence the corresponding empty row and column. 


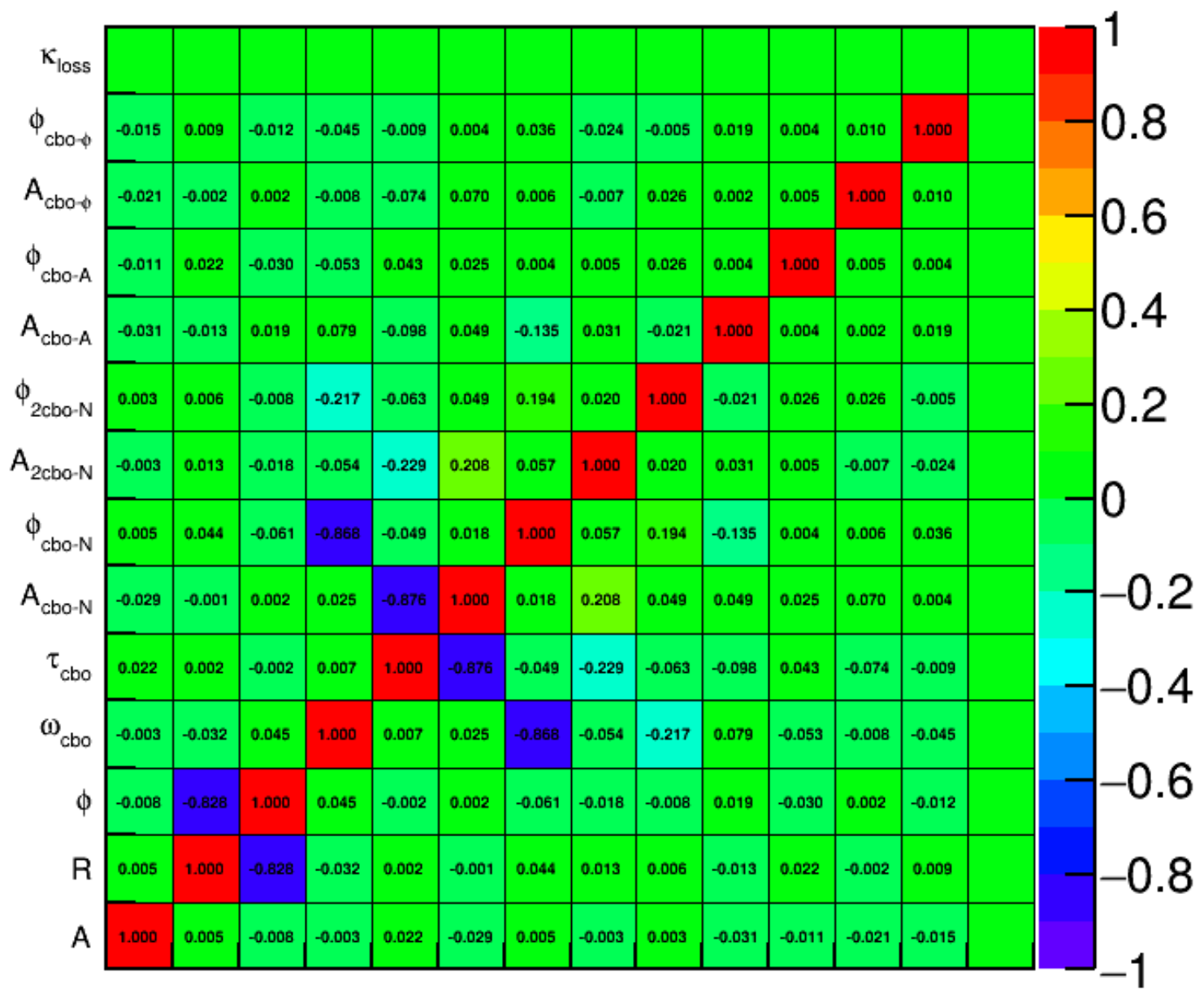

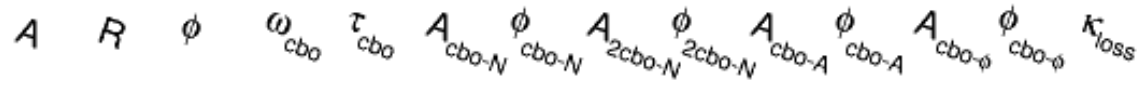

Figure D.4: Correlation matrix for the single seed ratio fit to the Endgame dataset. The only significant correlation with $R$ is the $g-2$ phase. $\kappa_{\text {loss }}$ is fixed, hence the corresponding empty row and column. 


\section{Appendix E}

\section{Ratio Method - T-Method Randomization Comparison}

The final value of $\omega_{a}$ and by extension $a_{\mu}$ will be some type of combination or average between different analyzers and their different fit types. Ratio Method fits due to their additional level of randomization will result in fitted $R$ values that are consistent but different from T-Method fits to the same data. In order to assist in combining the Ratio Method results with T-Method results, it is desirable to understand the expected difference in the fitted $\omega_{a}$ value between the two fit types.

In order to investigate this a toy MC study was performed with 100 pseudoexperiments. Each pseudo-experiment consisted of a single set of "positron" data, generated using a 1D ROOT function corresponding to an energy threshold time histogram, described by the general five parameter function,

$$
N=N_{0} \cdot e^{-t / \tau} \cdot\left[1+A \cos \left(\omega_{a} t+\phi\right)\right] .
$$

The amount of statistics in each pseudo-experiment was chosen to be comparable to the $60 \mathrm{~h}$ dataset. This single set of hits was then time-randomized and ratiorandomized with 50 different random seeds, where the times of the generated hits were randomized in the same way as was done for data. In this way each pseudoexperiment corresponds to an idealized approximation of the $60 \mathrm{~h}$ dataset, with 50 different random seeds applied before fitting.

Five parameter fits and three parameter ratio fits were then performed on the 
histograms for all random seeds, and for all pseudo-experiments. The distribution of fitted $R$ values for a single pseudo-experiment is shown in Figure E.1a. While the true $R$ value is 0 as chosen in the simulation, the $R$ values for a single pseudo-experiment will be centered around some other value that is statistically consistent with 0 . (This is satisfied since the statistical error on $R$ is approximately $1.3 \mathrm{ppm}$.) Plots for all pseudo-experiment $R$ distribution means and widths are shown in Figures E·1b and $\mathrm{E} \cdot 1 \mathrm{c}$ respectively. In the former, the means of the plotted histograms are consistent with 0 , with widths corresponding to the statistical error on $R$. In the latter the widths of the T-Method and Ratio Method fits due to the randomization are shown. It can be seen that the Ratio Method, while having the same statistical precision as the T-Method, has a larger width due to the randomization of counts in the ratio histograms.

Finally, the differences in the mean of the $R$ distributions between the T-Method fit and the Ratio Method fit for each pseudo-experiment is plotted in Figure E.1d. It is the width of this histogram that is the number of interest. What this plot shows is that for a dataset with approximately the same statistics as the 60h dataset, an average $R$ value for 50 different random seeds for a T-Method fit that is $70 \mathrm{ppb}$ different from that for a ratio fit, is statistically consistent to $1 \sigma$. Table E.1 includes the expected differences for studies done with 10 times the statistics of the $60 \mathrm{~h}$ dataset, and with the VW randomization applied to the hit times. 


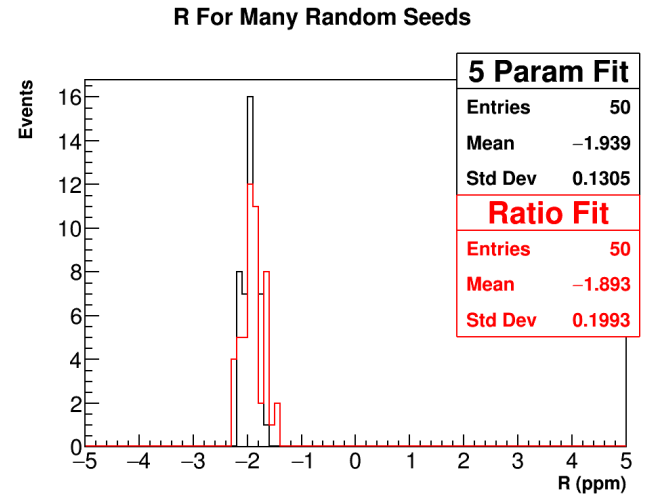

(a) Fitted $R$ distribution for a single pseudo-experiment for 5 parameter and 3 parameter ratio fits, for 50 different random seeds.

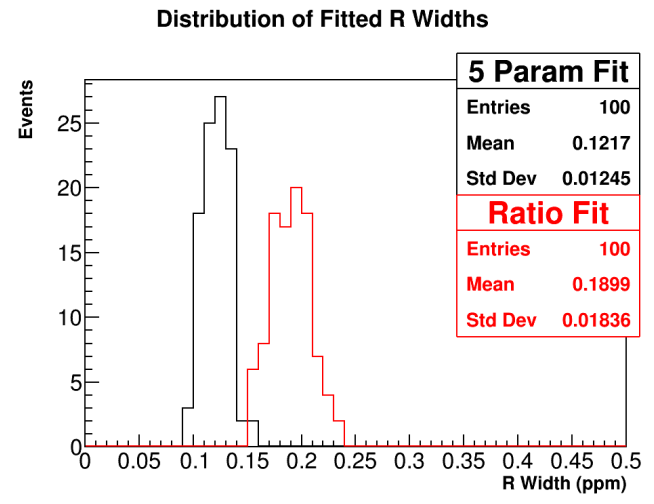

(c) The distribution of widths of the fitted $R$ distributions, for 100 separate pseudo-experiments.

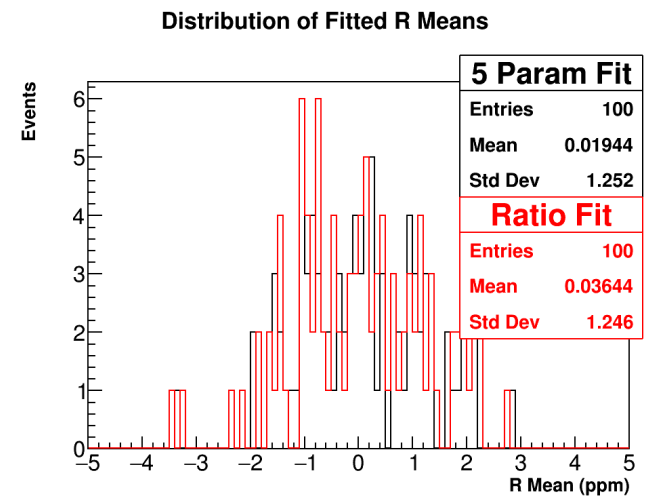

(b) The distribution of means of the fitted $R$ distributions, for 100 separate pseudoexperiments.

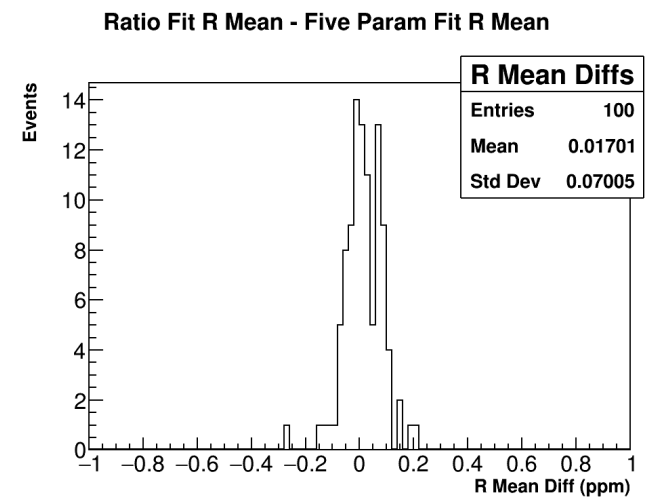

(d) The distribution of differences in fitted $R$ means between 5 parameter fits and 3 parameter ratio fits per pseudoexperiment for 100 pseudo-experiments.

Figure E·1: Toy MC study investigating differences in expected $R$ values between Ratio Method and T-Method fits due to different randomizations. The statistical precision on $R$ for a single fit is approximately $1.3 \mathrm{ppm}$. 


\begin{tabular}{lccc}
\hline \multicolumn{4}{c}{ T-R Method Randomization Comparison } \\
\hline \hline Stat level and randomization & Error on $R$ & Mean Difference RMS & Error on RMS \\
\hline$\sim 60 \mathrm{~h}$ stats & 1322.3 & 70.1 & 5.0 \\
$\sim 10 \times$ 60h stats & 416.4 & 29.3 & 2.1 \\
$\sim 60 \mathrm{~h}$ stats w/ VW randomization & 1374.9 & 84.6 & 6.0 \\
\hline
\end{tabular}

Table E.1: RMS of mean difference between $\mathrm{T}$ and R Method fits with different levels of statistics. In each case times are randomized by $\pm T_{c} / 2$ where $T_{c}$ is the cyclotron period or bin width. In the third row times are also randomized by $\pm T_{V W} / 2$ where $T_{V W}$ is the VW period, here set to $485.709 \mathrm{~ns}$ corresponding to an $n$ value of 0.120 . Units are in ppb. 


\section{List of Abbreviated Journal Titles}

Acta Phys. Polon.

Comput. Phys. Commun. Eur. Phys. J.

J. Phys. Conf. Ser. JHEP

JINST

Nucl. Instrum. Meth.

Phys. Rev.

Phys. Rev. Accel. Beams

Phys. Rev. Lett.

PoS

Rev. Mod. Phys.
Acta Physica Polonica

Computer Physics Communications

$\ldots \ldots \ldots \ldots . . \ldots$ European Physical Journal

.......... Journal of Physics: Conference Series

$\ldots \ldots \ldots \ldots$ Journal of High Energy Physics

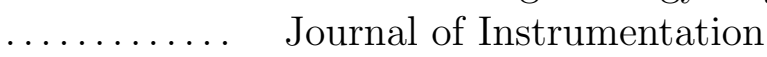

.......... Nuclear Instruments and Methods

$\ldots \ldots \ldots \ldots$ Physical Review

$\ldots . \ldots . \ldots$ Physical Review Accelerators and Beams

$\ldots \ldots \ldots \ldots$ Physical Review Letters

$\ldots . \ldots . \ldots . \quad$ Proceedings of Science

.......... Review of Modern Physics 


\section{Bibliography}

[1] P. Dirac. "The quantum theory of the electron". In: Proceedings of the Royal Society of London Series A, Containing Papers of a Mathematical and Physical Character (1928).

[2] M. Schwartz. Quantum Field Theory and the Standard Model. Cambridge University Press, 2014.

[3] J. E. Nafe, E. B. Nelson, and I. I. Rabi. "The Hyperfine Structure of Atomic Hydrogen and Deuterium". In: Phys. Rev. 71 (12 June 1947), pp. 914-915. DOI: 10.1103/PhysRev .71.914. URL: https ://link . aps . org/doi/10. 1103/PhysRev.71.914.

[4] Julian Schwinger. "On Quantum-Electrodynamics and the Magnetic Moment of the Electron". In: Phys. Rev. 73 (4 Feb. 1948), pp. 416-417. DoI: 10.1103/ PhysRev.73.416. URL: https://link.aps.org/doi/10.1103/PhysRev.73. 416.

[5] H. M. Foley and P. Kusch. "On the Intrinsic Moment of the Electron". In: Phys. Rev. 73 (4 Feb. 1948), pp. 412-412. DOI: 10.1103/PhysRev.73.412. URL: https://link.aps.org/doi/10.1103/PhysRev.73.412.

[6] P. Kusch and H. M. Foley. "The Magnetic Moment of the Electron". In: Phys. Rev. 74 (3 Aug. 1948), pp. 250-263. DOI: 10.1103/PhysRev.74.250, URL: https://link.aps.org/doi/10.1103/PhysRev.74.250.

[7] Joshua Ellis. "TikZ-Feynman: Feynman diagrams with TikZ". In: Comput. Phys. Commun. 210 (2017), pp. 103-123. DOI: 10.1016/j.cpc.2016.08.019. arXiv: 1601.05437 [hep-ph].

[8] Max Dohse. "TikZ-FeynHand: Basic User Guide". In: (2018). arXiv: 1802. 00689 [cs.0H].

[9] Tatsumi Aoyama et al. "Complete Tenth-Order QED Contribution to the Muon $g-2$ ". In: Phys. Rev. Lett. 109 (2012), p. 111808. DOI: 10.1103/ PhysRevLett.109.111808, arXiv: 1205.5370 [hep-ph].

[10] Tatsumi Aoyama, Toichiro Kinoshita, and Makiko Nio. "Revised and Improved Value of the QED Tenth-Order Electron Anomalous Magnetic Moment". In: Phys. Rev. D97.3 (2018), p. 036001. DOI: 10.1103/PhysRevD.97.036001. arXiv: 1712.06060 [hep-ph] 
[11] Tadashi Ishikawa, Nobuya Nakazawa, and Yoshiaki Yasui. "Numerical calculation of the full two-loop electroweak corrections to muon $(g-2)$ ". In: Phys. Rev. D99.7 (2019), p. 073004. DOI: 10.1103/PhysRevD .99.073004. arXiv: 1810.13445 [hep-ph].

[12] C. Gnendiger, D. Stöckinger, and H. Stöckinger-Kim. "The electroweak contributions to $(g-2)_{\mu}$ after the Higgs boson mass measurement". In: Phys. Rev. D88 (2013), p. 053005. DOI: 10.1103 / PhysRevD . 88 .053005, arXiv: 1306.5546 [hep-ph].

[13] The Muon $g-2$ Theory Initiative.

https://indico.fnal.gov/event/13795/, https://indico.him.uni-mainz.de/event/11/, http://www . int.washington. edu/PROGRAMS/19-74W/.

[14] Bernd A. Kniehl. "Dispersion relations in loop calculations". In: Acta Phys. Polon. B27 (1996), pp. 3631-3644. arXiv: hep-ph/9607255 [hep-ph].

[15] F. Jegerlehner. The Anomalous Magnetic Moment of the Muon. Springer Tracts in Modern Physics. Springer Berlin Heidelberg, 2007. ISBN: 9783540726340. URL: https://books .google.com/books?id=uxQj004Py6AC.

[16] Alexander Keshavarzi. "The muon $g-2$ : a brief overview of hadronic cross section data". In: 9th International Workshop on Chiral Dynamics (CD18) Durham, NC, USA, September 17-21, 2018.2019. arXiv: 1903.10349 [hep-ex].

[17] Alexander Keshavarzi, Daisuke Nomura, and Thomas Teubner. "Muon $g-$ 2 and $\alpha\left(M_{Z}^{2}\right)$ : a new data-based analysis". In: Phys. Rev. D97.11 (2018), p. 114025. DOI: 10.1103/PhysRevD.97.114025, arXiv: 1802.02995 [hep-ph].

[18] Alexander Keshavarzi, Daisuke Nomura, and Thomas Teubner. "The $g-2$ of charged leptons, $\alpha\left(M_{Z}^{2}\right)$ and the hyperfine splitting of muonium". In: (2019). arXiv: 1911.00367 [hep-ph].

[19] Michel Davier et al. "Reevaluation of the hadronic vacuum polarisation contributions to the Standard Model predictions of the muon $g-2$ and $\alpha\left(m_{Z}^{2}\right)$ using newest hadronic cross-section data". In: Eur. Phys. J. C77.12 (2017), p. 827. DOI: 10.1140/epjc/s10052-017-5161-6, arXiv: 1706.09436 [hep-ph].

[20] M. Davier et al. "A new evaluation of the hadronic vacuum polarisation contributions to the muon anomalous magnetic moment and to $\boldsymbol{\alpha}\left(\mathbf{m}_{\mathbf{Z}}^{\mathbf{2}}\right)$ ". In: (2019). arXiv: 1908.00921 [hep-ph].

[21] Alexander Kurz et al. "Hadronic contribution to the muon anomalous magnetic moment to next-to-next-to-leading order". In: Phys. Lett. B734 (2014), pp. 144-147. DOI: $10.1016 / \mathrm{j} \cdot$ physletb.2014.05.043 arXiv: 1403.6400 [hep-ph]. 
[22] T. Blum et al. "Calculation of the Hadronic Vacuum Polarization Contribution to the Muon Anomalous Magnetic Moment". In: Phys. Rev. Lett. 121 (2 July 2018), p. 022003. DOI: 10.1103 / PhysRevLett . 121.022003 . URL: https : //link.aps.org/doi/10.1103/PhysRevLett.121.022003.

[23] Joaquim Prades, Eduardo de Rafael, and Arkady Vainshtein. "The Hadronic Light-by-Light Scattering Contribution to the Muon and Electron Anomalous Magnetic Moments". In: Adv. Ser. Direct. High Energy Phys. 20 (2009), pp. 303-317. DOI:10.1142/9789814271844_0009, arXiv:0901.0306 [hep-ph].

[24] Martin Hoferichter et al. "Pion-Pole Contribution to Hadronic Light-By-Light Scattering in the Anomalous Magnetic Moment of the Muon". In: Phys. Rev. Lett. 121 (11 Sept. 2018), p. 112002. DOI: 10.1103/PhysRevLett.121.112002. URL: https://link.aps.org/doi/10.1103/PhysRevLett.121.112002.

[25] Pere Masjuan and Pablo Sanchez-Puertas. "Pseudoscalar-pole contribution to the $\left(g_{\mu}-2\right)$ : A rational approach". In: Phys. Rev. D 95 (5 Mar. 2017), p. 054026. DOI: 10.1103/PhysRevD .95.054026. URL: https://link .aps . org/doi/10.1103/PhysRevD.95.054026.

[26] Martin Hoferichter et al. "Dispersion relation for hadronic light-by-light scattering: pion pole". In: JHEP 10 (2018), p. 141. DOI: 10.1007/JHEP10(2018) 141. arXiv: 1808.04823 [hep-ph].

[27] Gilberto Colangelo et al. "Dispersion relation for hadronic light-by-light scattering: theoretical foundations". In: JHEP 09 (2015), p. 074. DOI: 10.1007/ JHEP09 (2015) 074, arXiv: 1506.01386 [hep-ph].

[28] Gilberto Colangelo et al. "Dispersion relation for hadronic light-by-light scattering: two-pion contributions". In: JHEP 04 (2017), p. 161. DOI: 10.1007/ JHEP04(2017)161, arXiv: 1702.07347 [hep-ph].

[29] Gilberto Colangelo et al. "Rescattering Effects in the Hadronic-Light-by-Light Contribution to the Anomalous Magnetic Moment of the Muon". In: Phys. Rev. Lett. 118 (23 June 2017), p. 232001. DOI: 10.1103/PhysRevLett.118. 232001. URL: https : //link . aps .org/doi/10.1103/PhysRevLett.118. 232001 .

[30] Gilberto Colangelo et al. "Longitudinal short-distance constraints for the hadronic light-by-light contribution to $(g-2)_{\mu}$ with large- $N_{c}$ Regge models". In: (2019). arXiv: 1910.13432 [hep-ph].

[31] Gilberto Colangelo et al. "Short-distance constraints on hadronic light-by-light scattering in the anomalous magnetic moment of the muon". In: (2019). arXiv: 1910.11881 [hep-ph].

[32] Thomas Blum et al. "The hadronic light-by-light scattering contribution to the muon anomalous magnetic moment from lattice QCD". In: (2019). arXiv: 1911.08123 [hep-lat]. 
[33] Nils Asmussen et al. "Developments in the position-space approach to the HLbL contribution to the muon $g-2$ on the lattice". In: (2019). arXiv: 1911. 05573 [hep-lat].

[34] Gilberto Colangelo et al. "Remarks on higher-order hadronic corrections to the muon g-2". In: Phys. Lett. B735 (2014), pp. 90-91. DOI: 10 . 1016/ j . physletb.2014.06.012, arXiv: 1403.7512 [hep-ph].

[35] B. Lee Roberts. "The History of the Muon $(g-2)$ Experiments". In: 15th International Workshop on Tau Lepton Physics (TAU2018) Amsterdam, Netherlands, September 24-28, 2018. 2018. arXiv: 1811.06974 [hep-ex].

[36] G. W. Bennett et al. "Final Report of the Muon E821 Anomalous Magnetic Moment Measurement at BNL". In: Phys. Rev. D73 (2006), p. 072003. DoI: 10.1103/PhysRevD.73.072003, arXiv: hep-ex/0602035 [hep-ex].

[37] Peter J. Mohr, David B. Newell, and Barry N. Taylor. "CODATA recommended values of the fundamental physical constants: 2014". In: Rev. Mod. Phys. 88 (3 Sept. 2016), p. 035009. DOI: 10.1103/RevModPhys.88.035009. URL: https://link.aps.org/doi/10.1103/RevModPhys.88.035009.

[38] Andrzej Czarnecki and William J. Marciano. "Muon anomalous magnetic moment: A harbinger for "new physics"". In: Physical Review D 64.1 (June 2001). ISSN: 1089-4918. DOI: 10.1103/physrevd.64.013014. URL: http://dx.doi. org/10.1103/PhysRevD.64.013014.

[39] Kaoru Hagiwara, Kai Ma, and Satyanarayan Mukhopadhyay. "Closing in on the chargino contribution to the muon $g-2$ in the MSSM: Current LHC constraints". In: Physical Review D 97.5 (Mar. 2018). ISSN: 2470-0029. DOI: 10.1103/physrevd.97.055035. URL: http://dx . doi . org/10 . 1103/ PhysRevD.97.055035.

[40] Estefania Coluccio Leskow et al. " $(g-2)_{\mu}$, lepton flavor violation, and $Z$ decays with leptoquarks: Correlations and future prospects". In: Physical Review D 95.5 (Mar. 2017). ISSN: 2470-0029. DOI: 10.1103/physrevd.95.055018. URL: http://dx.doi.org/10.1103/PhysRevD.95.055018.

[41] Andreas Crivellin, Dario Müller, and Christoph Wiegand. $b \rightarrow s \ell^{+} \ell^{-}$Transitions in Two-Higgs-Doublet Models. 2019. arXiv: 1903.10440 [hep-ph].

[42] Yoshihiko Abe, Takashi Toma, and Koji Tsumura. "A $\mu$ - $\tau$-philic scalar doublet under Zn flavor symmetry". In: Journal of High Energy Physics 2019.6 (June 2019). ISSN: 1029-8479. DOI: $10.1007 /$ jhep06 (2019) 142. URL: http://dx. doi.org/10.1007/JHEP06(2019) 142.

[43] D. Hanneke, S. Fogwell, and G. Gabrielse. "New Measurement of the Electron Magnetic Moment and the Fine Structure Constant". In: Phys. Rev. Lett. 100 (2008), p. 120801. DOI: 10.1103/PhysRevLett.100.120801. arXiv: 0801. 1134 [physics.atom-ph]. 
[44] Richard H. Parker et al. "Measurement of the fine-structure constant as a test of the Standard Model". In: Science 360.6385 (2018), pp. 191-195. ISSN: 0036-8075. DOI: 10 . 1126 / science . aap7706. eprint: https : / / science. sciencemag . org / content / 360/6385/191 . full . pdf. URL: https : / / science.sciencemag.org/content/360/6385/191.

[45] Andreas Crivellin, Martin Hoferichter, and Philipp Schmidt-Wellenburg. "Combined explanations of $(g-2)_{\mu, e}$ and implications for a large muon EDM". In: Phys. Rev. D 98 (11 Dec. 2018), p. 113002. DoI: 10.1103/PhysRevD . 98. 113002. URL: https://link.aps.org/doi/10.1103/PhysRevD.98.113002.

[46] Chien-Yi Chen et al. "Implications of a light "dark Higgs" solution to the $g_{\mu}-2$ discrepancy". In: Phys. Rev. D93.3 (2016), p. 035006. DOI: 10.1103/ PhysRevD.93.035006, arXiv: 1511.04715 [hep-ph].

[47] M. Ablikim et al. "Dark Photon Search in the Mass Range Between 1.5 and $3.4 \mathrm{GeV} / c^{2}$ ". In: Phys. Lett. B774 (2017), pp. 252-257. DOI: 10 . 1016 / j . physletb.2017.09.067, arXiv: 1705.04265 [hep-ex].

[48] W. J. Marciano et al. "Contributions of axionlike particles to lepton dipole moments". In: Physical Review D 94.11 (Dec. 2016). ISSN: 2470-0029. DOI: 10.1103/physrevd.94 . 115033. URL: http://dx . doi . org/10 . 1103/ PhysRevD.94.115033.

[49] M. Lancaster. Personal communication. 2019.

[50] J. Jackson. Classical Electrodynamics. Wiley Indiana, 2011.

[51] W. Liu et al. "High Precision Measurements of the Ground State Hyperfine Structure Interval of Muonium and of the Muon Magnetic Moment". In: Phys. Rev. Lett. 82 (4 Jan. 1999), pp. 711-714. DOI: 10.1103/PhysRevLett.82.711. URL: https://link.aps .org/doi/10.1103/PhysRevLett.82.711.

[52] E. D. Commins and P. H. Bucksbaum. Weak interactions of leptons and quarks. Cambridge University Press, 1983.

[53] G. W. Bennett et al. "Statistical equations and methods applied to the precision muon $(g-2)$ experiment at BNL". In: Nucl. Instrum. Meth. A579 (2007), pp. 1096-1116. DOI: $10.1016 /$ j.nima.2007.06.023.

[54] J. Grange et al. "Muon $(g-2)$ Technical Design Report". In: (2015). arXiv: 1501.06858 [physics.ins-det].

[55] R. Osofsky. Improving Magnetic Field Uniformity in the Muon $g-2$ Storage Ring. Muon $g-2$ DocDB 12497. 2018.

[56] D. Flay and D. Kawall. The High-Precision Calibration NMR Magnetometer for the Muon $g-2$ Experiment at Fermilab. Muon $g-2$ Note 192 DocDB 17818. 2018. 
[57] M. Tanabashi et al. "Review of Particle Physics". In: Phys. Rev. D 98 (3 Aug. 2018), p. 030001. DOI: 10.1103/PhysRevD .98.030001. URL: https: //link.aps.org/doi/10.1103/PhysRevD.98.030001.

[58] Diktys Stratakis et al. "Accelerator performance analysis of the Fermilab Muon Campus". In: Phys. Rev. Accel. Beams 20.11 (2017), p. 111003. DOI: 10.1103/ PhysRevAccelBeams.20.111003, arXiv: 1803.00597 [physics.acc-ph].

[59] C. Yoshikawa et al. "Optimization of the Target Subsystem for the New $g-$ 2 Experiment". In: Conference Proceedings C1205201 (2012), pp. 460-462. arXiv: 1301.6800 [physics.acc-ph].

[60] A. Yamamoto et al. "The superconducting inflector for the BNL $g-2$ experiment". In: Nucl. Instrum. Meth. A491 (2002), pp. 23-40. DOI:10.1016/S01689002(02)01232-9.

[61] Y. K. Semertzidis et al. "The Brookhaven muon $(g-2)$ storage ring high voltage quadrupoles". In: Nucl. Instrum. Meth. A503 (2003), pp. 458-484. DOI: $10.1016 / \mathrm{S} 0168-9002(03) 00999-9$.

[62] H. Wiedermann. Particle Accelerator Physics. Springer-Verlag New York, 1993.

[63] D. Seleznev, A. Chapelain, and D. Rubin. Extraction of the Muon Beam Frequency Distribution via the Fourier Analysis of the Fast Rotation Signal. Muon $g-2$ Note 130 DocDB 9701. 2018.

[64] A. Keshavarzi. Fast rotation summary. Muon $g-2$ DocDB 15304. 2018.

[65] M. J. Syphers. Storage Ring Linear Tune Tables. Muon $g-2$ DocDB 11547. 2018.

[66] A. Chapelain. Data Quality Control - a.k.a. DQC. Muon g-2 DocDB 15295. 2018.

[67] H. Binney et al. Data Quality Control for the 60-Hour Dataset. Muon $g-2$ DocDB 16568. 2019.

[68] K. Khaw. How each dataset is different for Run 1 of E989. Muon $g-2$ DocDB 15781. 2019.

[69] H. Binney. T0: update and current work. Muon $g-2$ DocDB 10162. 2018.

[70] A. Fienberg. T0 Detector. Muon $g-2$ DocDB 10911. 2018.

[71] P. Kammel et al. Inflector Beam Monitoring System Justification Document. Muon $g-2$ DocDB 2722. 2015.

[72] B. MacCoy and P. Kammel. IBMS Update. Muon $g-2$ DocDB 10944. 2018.

[73] F. Gray. Fiber Harp Beam Monitor. Muon $g-2$ DocDB 8366. 2017.

[74] P. Girotti et al. The Short-Time Double-Pulse correction. Muon $g-2$ Note 190 DocDB 17668. 2019. 
[75] E. Bottailco et al. Run 1 In-Fill Gain Correction. Muon $g-2$ Note 193 DocDB 17827. 2019.

[76] A. T. Fienberg et al. "Studies of an array of $\mathrm{PbF}_{2}$ Cherenkov crystals with large-area SiPM readout". In: Nucl. Instrum. Meth. A783 (2015), pp. 12-21. DOI: 10.1016/j.nima.2015.02.028, arXiv: 1412.5525 [physics.ins-det].

[77] J. Kaspar et al. "Design and performance of SiPM-based readout of $\mathrm{PbF}_{2}$ crystals for high-rate, precision timing applications". In: JINST 12.01 (2017), P01009. DOI: 10 .1088/1748-0221/12/01/P01009, arXiv: 1611.03180 [physics.ins-det].

[78] David A. Sweigart. "A new MicroTCA-based waveform digitizer for the Muon g-2 experiment". In: PoS ICHEP2016 (2016), p. 845. DOI: 10.22323/1.282. 0845, arXiv: 1612.05145 [physics.ins-det].

[79] A. Anastasi et al. "The laser control of the muon $g-2$ experiment at Fermilab". In: JINST 13.02 (2018), T02009. DOI: 10.1088/1748-0221/13/02/T02009.

[80] S. Charity. "Beam profile measurements using the straw tracking detectors at the Fermilab muon $g-2$ experiment, and a study of their sensitivity to a muon electric dipole moment". PhD thesis. University of Liverpool, 2018.

[81] W. Blum, W. Riegler, and L. Rolandi. Particle Detection with Drift Chambers. Springer-Verlag Berlin Heidelberg, 2008.

[82] M. Raminsky. Garfield. Muon $g-2$ DocDB 905. 2012.

[83] J. Mott. Garfield Paramterisation: First Simulation Attempt. Muon $g-2$ DocDB 6171. 2017.

[84] W. Turner. "The construction and commissioning of the straw tracking detector in the new muon $g-2$ experiment at Fermilab". PhD thesis. University of Liverpool, 2018.

[85] C. Green et al. "The Art Framework". In: J. Phys. Conf. Ser. 396 (2012), p. 022020. DOI: 10.1088/1742-6596/396/2/022020.

[86] T. Walton. Track Finding Overview. Muon $g-2$ DocDB 7580. 2017.

[87] T. Walton and J. Price. Track formation details. Muon $g-2$ DocDB 8555. 2017.

[88] J. Price. Track Finding - simple pattern recognition. Muon $g-2$ DocDB 5793. 2017.

[89] N. Kinnaird. Geane Track Fitting. Muon $g-2$ Note 184 DocDB 8102. 2017.

[90] V. Innocente, M. Maire, and E. Nagy. "GEANE: Average tracking and error propagation package". In: MC 91: Detector and event simulation in highenergy physics. Proceedings, Workshop, Amsterdam, Netherlands, April 8-12, 1991. 1991, pp. 58-78. URL: http://innocentonnice.web.cern.ch/innocentonnice/ napoli99/geane_manual.ps. 
[91] Lia Lavezzi. "The fit of nuclear tracks in high precision spectroscopy experiments". pp. 57-86. PhD thesis. Pavia U., 2007. URL: http://bamboo.pv . infn.it/doc/L_Lavezzi.pdf.

[92] K. Lassila-Perini and L. Urban. "Energy loss in thin layers in GEANT". In: Nucl. Instrum. Meth. A362 (1995), pp. 416-422. DOI: 10.1016/0168-9002(95) 00344-4.

[93] A. Strandlie and W. Wittek. "Derivation of Jacobians for the propagation of covariance matrices of track parameters in homogeneous magnetic fields". In: Nucl. Instrum. Meth. A566 (2006), pp. 687-698.

[94] V. Innocente and E. Nagy. "Trajectory fit in presence of dense materials". In: Nucl. Instrum. Meth. A324 (1993), pp. 297-306. DOI: 10 .1016/01689002(93) 90992-Q.

[95] J. Mott, N. Kinnaird, and F. Azfar. Residuals, Covariance \& Correlation in Fitting Straight Line Tracks in UV. Muon $g-2$ DocDB 3813. 2016.

[96] Gaël Guennebaud, Benoît Jacob, et al. Eigen v3. http://eigen.tuxfamily.org. 2010 .

[97] N. Kinnaird. Left/Right - What we do/have at the moment. Muon $g-2$ DocDB 12017. 2018.

[98] J. Price. LR from wire fit. Muon $g-2$ DocDB 6947. 2017.

[99] J. Price and S. Charity. Extrapolation uncertainty. Muon g- 2 DocDB 14920. 2018.

[100] J. Mott. Track Quality Cuts. Muon $g-2$ DocDB 16282. 2019.

[101] J. Mott. Track Quality Cuts: Second Edition. Muon g-2 DocDB 16420. 2019.

[102] J. Price. Vertical width and position - with and without fitting. Muon $g-2$ Elogs Tracking Analysis ID 187. 2019.

[103] J. Mott et al. CBO Frequency Change. Muon $g-2$ DocDB 14208. 2018.

[104] A. Fienberg. "Measuring the Precession Frequency in the E989 Muon $g-2$ Experiment". PhD thesis. University of Washington, 2019.

[105] W. Gohn. "Data Acquisition with GPUs: The DAQ for the Muon $g-2$ Experiment at Fermilab". In: PoS ICHEP2016 (2016), p. 174. DOI: 10.22323/ 1.282.0174, arXiv: 1611.04959 [physics.ins-det].

[106] Jason Crnkovic et al. "Lost Muon Study for the Muon $g-2$ Experiment at Fermilab". In: Proceedings, 8th International Particle Accelerator Conference (IPAC 2017): Copenhagen, Denmark, May 14-19, 2017. 2017, WEPIK119. DOI: $10.18429 /$ JACoW-IPAC2017-WEPIK119.

[107] C. Ozben and Y. Semertzidis. Eliminating Pileup from the g- 2 Data. E821 Muon $g-2$ Note 365. 2000. 
[108] J. Kindem. "The anomalous magnetic moment of the positive muon". PhD thesis. University of Minnesota, 1998.

[109] L. Duong. "A Precise Measurement of the Anomalous Magnetic Moment of the Positive Muon". PhD thesis. University of Minnesota, 2001.

[110] J. Paley. "Measurement of the Anomalous Magnetic Moment of the Negative Muon to 0.7 Parts Per Million". PhD thesis. Boston University, 2004.

[111] K. Labe. E989 Clock System Manual. 0.1. Muon g-2 DocDB 10995 v2. Cornell University. Mar. 2018.

[112] L. Gibbons. Blinders: $C++$ class for blinding fits. Muon $g-2$ DocDB 11232. 2018.

[113] J. Mott. CBO Frequency during EndGame dataset. Muon $g-2$ Elogs Tracking Analysis ID 184. 2019.

[114] J. Mott. Personal communication. 2019.

[115] N. Kinnaird and J. Mott. The Vertical Waist in the Ratio Method Analysis. Muon $g-2$ Note 214 DocDB 19466. 2019.

[116] T. Halewood-Leagas and J. Price. Vertical Betatron Oscillations. Muon $g-2$ DocDB 12894. 2018.

[117] S. Ganguly. Muon Losses - an Overview. Muon $g$ - 2 DocDB 12912. 2018.

[118] K. Labe. Studying Muon Losses with Recon East. Muon $g-2$ Note 188 DocDB 17695. 2019.

[119] N. Kinnaird. Analysis Note for 60H Dataset Relative Unblinding. Muon $g-2$ Note 172 DocDB 16270. 2019.

[120] K. Khaw. Notebook and plots for the 60 h relative unblinding ceremony. Muon $g-2$ DocDB 16667. 2019.

[121] A. Driutti. Personal communication. 2019.

[122] A. Driutti. Run 1 Production Update. Muon $g-2$ DocDB 19856. 2019.

[123] H. Binney. Lost muon measurements Run 1 and Run 2. Muon $g-2$ DocDB 20297. 2019.

[124] M. Syphers. Some Notes on Lost Muon Systematic Error. Muon $g-2$ DocDB 17617. 2019.

[125] S. Ganguly. Muon Loss Systematic Error. Muon $g-2$ DocDB 18082. 2019.

[126] J. Crnkovic et al. Differential decay systematic error in muon g-2 experiments. Muon $g-2$ DocDB 3693. 2016.

[127] J. Mott and J. Price. Tracker Measurement of $y^{2}$. Muon $g-2$ DocDB 17539. 2019. 
[128] J. Mott. Pitch Correction: Tracker Measurements. Muon g-2 DocDB 18094. 2019 .

[129] A. Chapelain et al. Fast rotatation analysis of the Run-1 60-hour data set with the Cornell Fourier method. Muon $g-2$ Note 211 DocDB 19150. 2019.

[130] A. Chapelain et al. Fast rotatation analysis of the Run-1 9-day data set with the Cornell Fourier method. Muon $g$ - 2 Note 212 DocDB 19252. 2019.

[131] A. Chapelain et al. Fast rotatation analysis of the Run-1 End-game data set with the Cornell Fourier method. Muon $g-2$ Note 213 DocDB 19258. 2019.

[132] D. Rubin, A. Chapelain, and J. Fagin. Corrections for Electric Field and Pitch. Muon $g-2$ DocDB 18085. 2019.

[133] D. Rubin. E - Field/Pitch alignment/voltage error systematic (update). Muon $g-2$ DocDB 18085. 2019.

[134] V. Tishchenko. Acceptance-Phase workshop summary. Muon $g-2$ DocDB 20568. 2019.

[135] W. Morse and V. Tishchenko. 60-hour Vertical Beta Function Change Early to Late. Muon $g-2$ DocDB 20279. 2019.

[136] F. Farley et al. Estimation of Error in Differential Pileup Subtracted Data. E821 Muon $g-2$ Note 377. 2000. 


\section{Nicholas Kinnaird}

\section{Education}

Ph.D. in Physics

Boston University
Expected 2020

Boston, MA

Dissertation: Muon Spin Precession Frequency Extraction and Decay Positron Track

Fitting in Run 1 of the Fermilab Muon $g-2$ Experiment

M.A. in Physics

Boston University

Boston, MA

B.S. in Physics

University of Texas at Austin

2013

Austin, TX

B.S. in Mathematics

2013

University of Texas at Austin

Austin, TX

\section{Projects}

- Track Fitting: Developed code to fit decay positron tracks in the E989 experiment. The algorithm was implemented into a modular $\mathrm{C}++$ framework to interface with upstream and downstream modules of the track reconstruction code. The algorithm fit tracks by propagating particles in a Geant 4 simulation and doing a $\chi^{2}$ minimization with track representation matrix objects.

- Frequency Extraction: Performed frequency analysis of Run 1 data of the E989 experiment. The project involved writing code to extract a frequency from histograms to high precision by fitting functions. The code was generalized to allow fitting different subsets of the data, or with different fit parameters, in order to systematically evaluate the data and verify the integrity of the results.

- Magnetic Field Analysis: Measured and analyzed external magnetic fields in the E989 experimental hall before construction using a fluxgate magnetometer. Simulated and analyzed the E989 magnetic ring storage and fringe field in Opera 2D and Opera $3 \mathrm{D}$.

\section{Technical Skills}

$\begin{array}{ll}\text { Programming Languages } & \mathrm{C}++, \mathrm{C}, \text { Python, LaTex, Bash } \\ \text { Software } & \text { Mathematica, Opera 2D, Opera 3D, Geant4, } \\ & \text { ROOT, git, Paraview, Linux, OSX, Windows } \\ \text { General } & \text { Collaboration, Presentation, Technical Writing, } \\ & \text { Data Analysis, Hardware Testing, Tutoring }\end{array}$

